\title{
Inhomogeneous Chiral Condensates in Low-Energy Color-Superconductivity Models of QCD
}

\author{
Dissertation \\ zur Erlangung des Doktorgrades \\ der Naturwissenschaften
}

vorgelegt beim Fachbereich Physik

der Johann Wolfgang Goethe-Universität

in Frankfurt am Main

\author{
von \\ Phillip Lakaschus \\ aus Fulda, Hessen
}

Frankfurt am Main 2021

D30 
vom Fachbereich Physik der

Johann Wolfgang Goethe-Universität als Dissertation angenommen.

Dekan: Prof. Dr. Harald Appelshäuser

Gutachter: Prof. Dr. Dirk H. Rischke

Prof. Dr. Jürgen Schaffner-Bielich

Datum der Disputation: 30.08.2021 
"The world is not magic - and that's the most magical thing about it."

Sean M. Carrol 



\section{Contents}

Deutsche Zusammenfassung ix

$\begin{array}{ll}\text { Abstract } & \mathbf{x v}\end{array}$

1 Introduction 1

2 Quantum Chromodynamics and

Low-Energy Effective Models 7

2.1 Quantum Chromodynamics . . . . . . . . . . . . . . 7

Asymptotic Freedom and Confinement . . . . . . . 9

Chiral Symmetry . . . . . . . . . . . . . . . . . . . 9

Color Superconductivity . . . . . . . . . . . . . . . . 12

2.2 Low-Energy Models of QCD . . . . . . . . . . . . . . . . . . 14

2.3 The Nambu-Jona-Lasinio Model . . . . . . . . . . . . . . . . . 16

2.4 The Quark-Meson Model . . . . . . . . . . . . . . . . . . . . . . 18

2.5 The Quark-Meson-Diquark Model . . . . . . . . . . . . . . . . 23

3 Competition of Inhomogeneous Chiral Condensates with Color Superconductivity in the Mean-Field Approximation 25

3.1 Grand Potential . . . . . . . . . . . . . . . . . 26

3.1.1 Inhomogeneous Chiral Condensates . . . . . . . . . 29

Chiral Density Wave . . . . . . . . . . . . . . . . . 29

Real-Kink Crystal . . . . . . . . . . . . . . . . . . 31

Regularization and Model Parameters . . . . . . . . 32

3.2 Results ........................... 33

4 The Functional Renormalization Group $\quad 39$

4.1 The Concept . . . . . . . . . . . . . . . . . . 39

4.2 The Exact Renormalization Group Equation . . . . . . . . . . . 41

4.3 Truncations .................... 45

4.4 Flow of the Effective Potential . . . . . . . . . . . . . . . 47

4.5 Flow of the Two-Point Function and Stability Analysis . . . . 51

5 Numerical Solution Details $\quad 53$

5.1 Finite-Volume Method . . . . . . . . . . . . . . . . . . 54

5.2 Numerical Tests . . . . . . . . . . . . . . . . . . 56

5.2.1 Numerical Tests: Quark-Meson Model . . . . . . . . . . 57

5.2.2 Numerical Tests: Quark-Meson-Diquark Model . . . . 62 
$\begin{array}{lll}6 & \text { Results } & 69\end{array}$

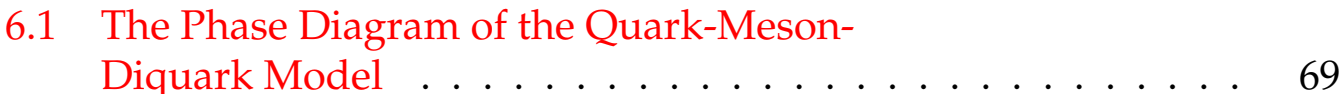

6.2 Stability Analysis . . . . . . . . . . . . . . 76

6.3 Renormalization-Group Consistent UV Potentials . . . . . . . 80

$\begin{array}{llrl}7 & \text { Conclusions and Outlook } & 83\end{array}$

$\begin{array}{lr}\text { A Notation and Conventions } & \mathbf{8 7}\end{array}$

A.1 Units . . . . . . . . . . . . . . . . . 87

A.2 Minkowski and Euclidean Spacetime . . . . . . . . . . . 87

A.3 Momentum Integral at Finite Temperature . . . . . . . . . . 88

A.4 Dirac Algebra . . . . . . . . . . . . . . 88

B Attractive Diquark Channel 91

C Mean-Field Study Appendices $\quad 95$

C.1 Hubbard-Stratonovich Transformation . . . . . . . . . . . 95

C.2 Trace $\log \ldots \ldots . \ldots$. . . . . . . . . . . . 96

C.3 Square Hamiltonian . . . . . . . . . . . . . . . . . . . . 97

D Flow of the Effective Potential 99

D.1 Bosonic Contribution . . . . . . . . . . . . . . . 99

D.2 Fermionic Contribution . . . . . . . . . . 106

E Flow of the Two-Point Function at

$\begin{array}{ll}\text { Finite External Momenta } & 113\end{array}$

E.1 Wetterich equation for the two-point function . . . . . . . . . . 113

E.2 Simplification of the Two-Point Function for Finite External Momentum .................... 116

E.3 Flow of the Two-Point Function for the Quark-Meson-Model . 117

E.4 Flow of the Two-Point Function for the

Quark-Meson-Diquark-Model . . . . . . . . . . . . . . . . . 122

F Code Reference $\quad 125$

F.1 Minimization of the NJL-model Grand Potential . . . . . . . . 125

F.2 Effective Potential of the QM model . . . . . . . . . . . . 125

F.3 Effective Potential of the QMD model . . . . . . . . . . . . 125

F.4 Derivation of the Flow Equations for the Effective Potential and the Two-Point Function for the QMD Model . . . . . . . . 126

$\begin{array}{lr}\text { Bibliography } & 127\end{array}$

$\begin{array}{ll}\text { Contribution to Chapter } 3 & 137\end{array}$

$\begin{array}{ll}\text { Danksagung } & 139\end{array}$ 


\section{List of Abbreviations}

$\begin{array}{ll}\text { CDW } & \text { Chiral Density Wave } \\ \text { CFL } & \text { Color-Flavor Locking } \\ \text { EFT } & \text { Effective Field Theory } \\ \text { ELSM } & \text { Extended Linear Sigma Model } \\ \text { FDM } & \text { Finite Difference Method } \\ \text { FRG } & \text { Functional Renormalization Group } \\ \text { FVM } & \text { Finite Volume Method } \\ \text { GN } & \text { Gross-Neveu } \\ \text { IR } & \text { Infrared } \\ \text { LEFT } & \text { Low-Energy Effective Field Theory } \\ \text { LFT } & \text { Lattice Field Theory } \\ \text { LQCD } & \text { Lattice Quantum Chromodynamics } \\ \text { LSM } & \text { Linear Sigma Model } \\ \text { LPA } & \text { Local Potential Approximation } \\ \text { MFT } & \text { Mean-Field Theory } \\ \text { NG } & \text { Nambu-Gorkov } \\ \text { NJL } & \text { Nambu-Jona-Lasinio } \\ \text { QCD } & \text { Quantum Chromodynamics } \\ \text { QFT } & \text { Quantum Field Theory } \\ \text { QM } & \text { Quark-Meson } \\ \text { QMD } & \text { Quark-Meson-Diquark } \\ \text { RKC } & \text { Real-Kink Crystal } \\ \text { UV } & \text { Ultraviolet } \\ \text { 2SC } & \text { Two-Flavor Color Superconductivity }\end{array}$





\section{Deutsche Zusammenfassung}

Die Existenz der Starken Wechselwirkung ist alles andere als augenscheinlich, da sie in unserem von elektromagnetischen und gravitativen Kräften geprägten Alltag nicht unmittelbar sichtbar ist. Stellt man sich jedoch die Frage, was eigentlich die kleinsten Bausteine unserer Welt, die Atomkerne, zusammenhält, so kommt man nach reiflicher Überlegung zu dem Schluss, dass weder Gravitation noch Elektromagnetismus die Stabilität von Atomkernen erklären können. Für Elemente mit Kernladungszahl zwei und größer verhindert die elektromagnetische Wechselwirkung die Entstehung von stabilen Atomkernen, da die resultierende abstoßende Kraft zwischen zwei gleich geladenen Teilchen bei solch kleinen Abständen enorm ist. Gravitation dagegen ist um 36 Größenordnungen schwächer als die elektromagnetische Kraft und daher kann auch die kleine gravitative Anziehung zweier Protonen keine Stabilität garantieren. Das ist der Punkt, an dem die Quantenchromodynamik (QCD) ins Spiel kommt. Die QCD ist die fundamentale Theorie zur Beschreibung der Starken Wechselwirkung und sie kann die Existenz und Stabilität der Atomkerne erklären. Atomkerne bestehen aus Protonen und Neutronen, und diese wiederum bestehen aus jeweils drei sogenannten Quarks. Die Quarks besitzen ebenfalls elektrische Ladung und erklären damit die positive Ladung von Protonen, aber im Gegensatz zu Elektronen besitzen sie eine weitere Quantenzahl, nämlich die sogenannte Farbladung. Diese Farbladung macht sie empfänglich für die Starke Kraft, die durch die sogenannten Gluonen vermittelt wird. Die Starke Wechselwirkung führt dann zu einer effektiven attraktiven Nukleon-Nukleon-Wechselwirkung, die durch effektive mesonische Freiheitsgrade realisiert wird. Die attraktive Nukleon-NukleonWechselwirkung überwindet die abstoßende elektromagnetische Kraft und erklärt damit die Stabilität von Atomkernen.

Dieses Ergebnis ist allerdings alles andere als trivial, denn die tatsächlichen Details der starken Wechselwirkung sind von enormer Komplexität. Zum einen besitzt die Farbladung im Gegensatz zur elektrischen Ladung drei mögliche Werte, die rot, grün und blau genannt werden. Ein Zustand, der aus drei verschiedenfarbigen Quarks besteht, ist ein farbneutraler Zustand, sowie ein Zustand aus Farbe+Antifarbe einem farbneutralen Zustand entspricht. Wenn die Nettofarbladung eines Teilchens verschwindet, dann sagt man auch, dass das Teilchen weiß ist. In der Niederenergieregion der QCD, das ist der Bereich für Impulsüberträge $\lesssim 200 \mathrm{MeV}$, existieren Quarks und Gluonen nicht als freie Teilchen, sondern kommen nur in weißen Bindungzuständen vor. Dieses Phänomen nennt man Confinement und wurde aufgrund der mathematischen Komplexität der QCD bisher noch nicht endgültig verstanden. 
Eine weitere Schwierigkeit der QCD ist die sogenannte chirale Symmetriebrechung. In einem ebenfalls analytisch noch nicht vollständig verstandenen Prozess bilden quasi masselose Quarks ein chirales Kondensat, das die ursprünglich vorhandene chirale Symmetrie bricht, da sich die Quarks aufgrund der Drehimpulserhaltung in Paaren mit entgegengesetzter Chiralität anordnen. Das wiederum führt dazu, dass die Quarks mit dem Kondensat wechselwirken und dadurch eine effektive Masse erhalten. Dieses Phänomen erklärt den größten Teil der Masse von z.B. Protonen und Neutronen. Während die Up- und Down-Quarks Massen von jeweils weniger als $5 \mathrm{MeV}$ haben, so haben Protonen und Neutronen jedoch eine Masse von jeweils ungefähr $939 \mathrm{MeV}$. Die restliche fehlende Masse, in etwa 99\%, entstehen effektiv durch die Wechselwirkung mit dem chiralen Kondensat.

Es gibt außerdem noch eine weitere Schwierigkeit der QCD, nämlich das Phänomen der sogenannten asymptotischen Freiheit, die möglicherweise mit dem Confinement in Verbindung steht. Die asymptotische Freiheit bezeichnet das Phänomen, dass die Starke Wechselwirkung schwächer wird, je kleiner die Abstände der wechselwirkenden Quarks sind, und umgekehrt wird sie größer, je größer die Abstände werden. Das führt dazu, dass man in dem Energiebereich, in dem die Kopplung zu groß ist, keine störungstheoretische Näherung durchführen kann und das Problem somit nichtperturbativ wird. Das erhöht den Aufwand von analytischen Rechnungen drastisch, da im Prinzip unendlich viele Feynman-Diagramme aller Ordnungen berücksichtigt werden müssen.

Aufgrund der erläuterten Komplexität der QCD, ist es bis jetzt nicht gelungen, die QCD direkt zu lösen. Stattdessen kann man Niederenergiemodelle der QCD verwenden, die die Symmetrien der QCD realisieren, dabei aber weniger technische Schwierigkeiten bereiten. Das ist die Herangehensweise in dieser Doktorarbeit. Wir studieren dazu zwei unterschiedliche Niederenergiemodelle der QCD, das sogenannte Nambu-Jona-Lasinio-Modell und das Quark-Meson-Modell. Das Nambu-Jona-Lasinio-Modell enthält zunächst nur Konstituentenquarks. Die relevanten mesonischen Freiheitsgrade werden dann durch Bosonisierung der Quark-Quark-Wechselwirkung explizit in das Modell eingebaut. Das Quark-Meson-Modell hat den gleichen physikalischen Inhalt wie das bosonisierte Nambu-Jona-Lasinio-Modell, jedoch enthält es bereits auf dem Level der Lagrangedichte die kinetischen Terme für die mesonischen Freiheitsgrade und einen allgemeineren Potentialterm, die im Nambu-Jona-Lasinio-Modell erst durch weitere Quantenkorrekturen erzeugt werden. Beide Modelle können daher als unterschiedliche Trunkierungen der gleichen Niederenergietheorie aufgefasst werden.

Um die Welt um uns herum zu verstehen, reicht es nicht aus, die Eigenschaften von einzelnen isolierten Teilchen zu studieren, da diese üblicherweise in Ensembles auftreten und die Ensembles wiederum in verschiedenen Phasen in Erscheinung treten. Das bekannteste Beispiel aus dem Alltag ist wohl Wasser, das in fester, flüssiger und gasförmiger Form auftreten kann. Und es sind die kollektiven Eigenschaften von Wasser, die unseren Alltag prägen, nicht die Eigenschaften auf molekularer Ebene. Dies gilt in ähnlicher 
Weise auch für die Starke Wechselwirkung und deshalb ist es das Ziel dieser Arbeit, das Phasendiagramm der QCD besser zu verstehen. Ein besseres Verständnis des QCD-Phasendiagramms ist von enormer Bedeutung für die Erforschung von kompakten Sternen, dem frühen Universum, sowie Schwerionenkollisionen in Teilchenbeschleunigern.

Der Fokus dieser Arbeit liegt auf der Erforschung sogenannter inhomogener chiraler Phasen. Das sind Phasen, die sich durch ein räumlich variierendes chirales Kondensat auszeichnen, was man sich bildlich wie ein Kristall vorstellen kann. Eine weiteres Merkmal dieser Arbeit ist die Berücksichtigung von Farbsupraleitung für zwei Quark-Flavors. Dadurch, dass inhomogene chirale Phasen und farbsupraleitende Phasen in effektiven Niederenergiemodellen der QCD üblicherweise in der Nähe des chiralen Phasenübergangs auftreten, kommt es zu einem Wettbewerb beider Phasen. Dieser Wettbewerb führt zu drei verschiedenen Möglichkeiten: Erstens, die inhomogene chirale Phase ist gegenüber der farbsupraleitenden Phase energetisch bevorzugt und verdrängt die farbsupraleitende Phase. Zweitens, der umgekehrte Fall tritt ein. Die farbsupraleitende Phase ist gegenüber der inhomogenen chiralen Phase energetisch bevorzugt und verdrängt die inhomogene chirale Phase. Oder drittens, der energetisch günstigste Zustand ist erreicht, wenn beide Phasen koexistieren.

Wie bereits besprochen, verwenden wir das Nambu-Jona-Lasinio-Modell und das Quark-Meson Modell zur Untersuchung dieser Fragestellung. Und obwohl diese Modelle eine deutliche Vereinfachung zur fundamentaleren Theorie, der QCD, darstellen, ist das Lösen dieser Modelle ohne Näherung schlicht nicht möglich. Ein bekanntes Näherungsschema ist die sogenannte Mittelfeldnäherung. Bei der Mittelfeldnäherung linearisiert man die Wirkung der Theorie, indem man den fluktuierenden Anteil der bosonischen Freiheitsgrade vernachlässigt. Das führt dann zu einem effektiven Pfadintegral, das analytisch ausgewertet werden kann.

Diese Methode wenden wir in dieser Doktorarbeit auf das Nambu-JonaLasinio-Modell an, um so den Wettbewerb zwischen verschiedenen inhomogenen Ansätzen mit der two-flavor color-superconductivity (2SC) Phase zu untersuchen. Die 2SC Phase ist durch Spin-0 Cooperpaaren bestehend aus jeweils einem Up- und einem Down-Quark charakterisiert. Im Vergleich $\mathrm{zu}$ bereits existierenden Studien in diesem Bereich, haben wir in der vorliegenden Arbeit die Dispersionsrelation für einen allgemeinen Ansatz des inhomogenen chiralen Kondensates in Anwesenheit eines homogenen 2SCDiquark-Kondensats hergeleitet, für den Fall, dass die Dispersionsrelation des inhomogenen Ansatzes ohne Berücksichtigung der Farbsupraleitung bekannt ist. Diese Verallgemeinerung hat den Vorteil, dass wir bereits bekannte inhomogene Ansätze für den chiralen Ordnungsparameter ohne weitere Schwierigkeiten in unseren Formalismus einsetzen können. Wenn außerdem in Zukunft die Dispersionsrelationen weiterer inhomogener Ansätze gefunden werden, so können diese einfach in unseren Formalismus eingesetzt werden, um den Wettbewerb mit der 2SC-Phase zu studieren. 
Wir haben in diesem Formalismus zwei unterschiedliche inhomogene Ansätze des chiralen Kondensats untersucht, nämlich die sogenannte Chiral Density Wave und den sogenannten Real-Kink Crystal. In beiden Fällen finden wir für bestimmte Diquark-Kopplungen alle Phasen, die durch unseren Ansatz prinzipiell möglich sind: Eine homogene chirale Phase ohne Farbsupraleitung, eine inhomogene chirale Phase ohne Farbsupraleitung, eine Koexistenzphase mit inhomogenem chiralen Kondensat und homogener Farbsupraleitung, eine rein homogene farbsupraleitende Phase ohne chirale Kondensation, sowie eine vollständig restaurierte Phase, in der alle Ordnungsparameter verschwinden. Für größer werdende Diquark-Kopplungen wird die Koexistenzphase stetig kleiner und auch die rein inhomogene Phase wird verdrängt, während die farbsupraleitende Phase größer wird. Des Weiteren konnten wir in Übereinstimmung mit früheren Studien bestätigen, dass der Real-Kink Crystal auch in Anwesenheit von Fabrsupraleitung im Vergleich zur Chiral Density Wave energetisch bevorzugt ist.

Auch wenn die Ergebnisse der Studie in der Mittelfeldnäherung äußerst interessant sind, so haben sie vor allem einen qualitativen Charakter, da diese aufgrund der Vernachlässigung der bosonischen Fluktuationen eine grobe Näherung darstellen. Der nächste logische Schritt ist es daher, bosonische Fluktuationen systematisch zu berücksichtigen. Das ist das Thema des zweiten Teils dieser Doktorarbeit. Dazu verwenden wir den Formalismus der Funktionalen Renormierungsgruppe. Dies ist ein nichtstörungstheoretischer Formalismus, der nicht auf die Entwicklung in der Kopplungskonstanten beschränkt ist, sondern weitere Entwicklungsschemata der 1PI-Diagramme erlaubt. Wir wählen dazu die Ableitungsentwicklung in der niedrigsten Ordnung, die sogenannte Local Potential Approximation, da in der Literatur auf Basis dieses Trunkierungsschema bereits vielversprechende Ergebnisse erzielt wurden. Obwohl die Local Potential Approximation die einfachste Trunkierung der Ableitungsentwicklung darstellt, so handelt es sich um eine deutliche Verbesserung gegenüber herkömmlichen Studien in der Mittelfeldnäherung, da diese im Gegensatz zur Mittelfeldnäherung bosonische Fluktuationen beinhaltet.

Wir verwenden die Funktionale Renormierungsgruppe, um das Phasendiagramm des Quark-Meson-Diquark-Modells herzuleiten. Wir zeigen hier, dass die in der Publikation [1] auftretenden unphysikalischen negativen Entropiedichten verschwinden, wenn man 2SC-Diquarks berücksichtigt und die Diquark-Kopplung groß genug wählt. Das ist eine wichtige Erkenntnis, weil dadurch klar wird, dass es für eine thermodynamisch konsistente Beschreibung des Niederenergiebereichs der QCD wichtig ist, alle relevanten bosonischen Freiheitsgrade zu berücksichtigen. Anschließend haben wir eine Stabilitätsanalyse durchgeführt, um nach Hinweisen für die Existenz inhomogener chiraler Phasen im Quark-Meson-Diquark-Modell zu suchen. Wir haben dazu die skalenabhängige Pion-Zweipunktfunktion hergeleitet und untersucht, ob während des Flusses für bestimmte externe Impulse Nullstellen auftreten. Dabei konnten wir zeigen, dass die zuvor in Ref. [1] 
gefundene Region der Instabilität durch die Berücksichtigung der DiquarkFreiheitsgrade stark beeinflusst wird. Ähnlich wie im ersten Teil der vorliegenden Arbeit konnten wir zeigen, dass die farbsupraleitende Phase einen verdrängenden Effekt auf die Region der Instabilität ausübt: Je größer die Diquark-Kopplung, desto kleiner die Region der Instabilität. Des Weiteren finden wir auch einen Bereich im Phasendiagramm, indem sowohl eine Instabilität in der Pion-Zweipunktfunktion auftritt, als auch ein Diquark-Kondensat existiert. Das ist ein wichtiger Hinweis auf die mögliche Existenz einer Koexistenzphase, so wie wir sie in der Studie in der Mittelfeldnäherung gefunden haben. Auch wenn das Auffinden der Instabilität kein eindeutiger Indikator dafür ist, dass eine inhomogene chirale Phase im IR existiert, so dienen die in dieser Arbeit präsentierten Ergebnisse als wichtige Motivation, um weiter in diese Richtung zu forschen. 



\section{Abstract}

Institute for Theoretical Physics

\section{Inhomogeneous Chiral Condensates in Low-Energy Color-Superconductivity Models of QCD}

by Phillip LAKASCHUS

This thesis explores the phase diagrams of the Nambu-Jona-Lasinio (NJL) and quark-meson (QM) model in the mean-field approximation and beyond. The focus lies in the investigation of the interplay between inhomogeneous chiral condensates and two-flavor color superconductivity.

In the first part of this thesis, we study the NJL model with 2SC diquarks in the mean-field approximation and determine the dispersion relations for quasiparticle excitations for generic spatial modulations of the chiral condensate in the presence of a homogeneous 2SC-diquark condensate, provided that the dispersion relations in the absence of color superconductivity are known. We then compare two different Ansätze for the chiral order parameter, the chiral density wave (CDW) and the real-kink crystal (RKC). For both Ansätze we find for specific diquark couplings a so-called coexistence phase where both the inhomogeneous chiral condensate and the diquark condensate coexist. Increasing the diquark coupling disfavors the coexistence phase in favor of a pure diquark phase. On the other hand, decreasing the diquark coupling favors the inhomogeneous phase over the coexistence phase.

In the second part of this thesis the functional renormalization group is employed to study the phase diagram of the quark-meson-diquark model. We observe that the region of the phase diagram found in Ref. [1], where the entropy density takes on unphysical negative values, vanishes when including diquark degrees of freedom. Furthermore, we perform a stability analysis of the homogeneous phase and compare the results with those of Ref. [1]. We find that an increasing diquark coupling leads to a smaller region of instability as the 2SC phase extends to a smaller chemical potential. We also find a region where simultaneously an instability occurs and a nonvanishing diquark condensate forms, which is an indication of the existence of a coexistence phase in accordance with the results of the first part of this work. 



\section{Chapter 1}

\section{Introduction}

The exploration of the phase diagram of quantum chromodynamics (QCD) is one of the central subjects in contemporary high-energy physics. A better understanding of it has very significant implications for our understanding of the early Universe, where matter was very hot, and for compact stars, where matter is extremely dense.

With QCD we have found the theory of strong interactions, explaining the origin of most of the mass of visible matter in the form of protons and neutrons. These are made of electrically and color-charged particles, the socalled quarks, as well as the color-charged gauge bosons, the gluons, which act as a "glue" between the quarks, leading to the formation of bound states to protons and neutrons. QCD exhibits several interesting but also highly non-trivial phenomena that make its theoretical treatment very challenging. One phenomenon is the confinement of quarks inside hadrons, thus making it impossible to observe them as isolated particles. Another phenomenon is the generation of a chiral condensate $\langle\bar{\psi} \psi\rangle$, which in turn leads to a dynamical generation of effective quark masses as the quarks interact with the chiral condensate through gluon exchange and thereby make them effectively heavier. In this process, the chiral symmetry is spontaneously broken. Lastly, QCD is asymptotically free, i.e., as momentum transfer decreases the interaction strength increases and vice versa, and therefore perturbation theory breaks down at large distances and thus prevents us from accessing the lowenergy region analytically. This is the main reason why deriving the phase diagram from the theory of strong interactions is everything but simple.

To the rescue comes Lattice QCD (LQCD), which enables the numerical calculation of the path integral through Monte-Carlo simulations by discretizing spacetime. However, it is plagued by the infamous sign problem, which prevents the application of LQCD at arbitrary finite densities since the fermion determinant becomes complex at finite chemical potential and can therefore no longer be used as a probability weight in Monte-Carlo simulations. Advances in LQCD have made it possible to circumvent this problem to some degree, however, still only a small region of the phase diagram is accessible using LQCD.

A completely different approach is the study of effective low-energy models (LEMs) of QCD. These are models that try to mimic the most important features of QCD in the low-energy regime while being significantly easier to solve with analytical methods, such as the mean-field approximation (MFA) or the functional renormalization group (FRG). The reason they are easier 
to solve analytically is because they usually neglect the complex gluon dynamics completely. Sometimes not even quarks are included, but only the hadrons, e.g., pions and nucleons. In many such models it is comparatively easy to derive the phase diagram, but always with the caveat that no quantitative predictions for the QCD phase diagram can be made, since it is possible that the effective models do not accurately describe QCD in the low-energy region due to the missing gluon and current quark dynamics. Despite this caveat, the qualitative features derived from these models may very well also hold for QCD as hadrons are certainly the dominant degrees of freedom in the low-energy regime.

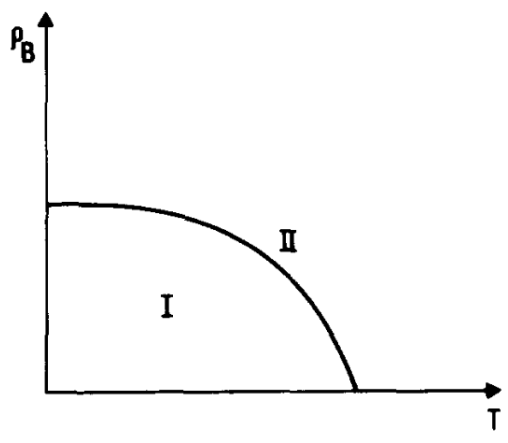

FIGURE 1.1: The QCD phase diagram proposed by N. Cabibbo and G. Parisi. $\rho_{\mathrm{B}}$ is the baryon density and $T$ the temperature. In phase I the quarks are confined in hadrons and in phase II they are deconfined. Figure taken from Ref. [2].
A very good example for this is the very first QCD model phase diagram which was published by N. Cabibbo and G. Parisi [2] in 1975, see Fig. 1.1. In this publication it has been recognized that at some critical temperature and density quarks are not confined anymore. The course of history was as follows: Rolf Hagedorn had published his statistical bootstrap hypothesis [3] in 1965, which states that heavier hadrons are made of lighter hadrons in a self-similar fashion. This model leads to an exponentially increasing spectrum of hadronic states, which comes with a critical temperature above which hadronic matter cannot be heated further. It should be noted that Hagedorn's model was invented in a time when it was not yet known that quarks are the constituents of hadrons. The insight that led N. Cabibbo and G. Parisi to the phase diagram in Fig. 1.1 was the following: When the hadron model is replaced by a quark confinement model, the previously obtained limiting temperature by Hagedorn becomes the critical temperature for a second-order phase transition for hadronic matter. Interestingly, Hagedorn's statistical bootstrap method yields a limiting temperature of about $\sim 154 \mathrm{MeV}$ [4], which is surprisingly close to the most up-to-date crossover transition temperature, $T \sim(156.5 \pm 1.5) \mathrm{MeV}$, obtained by LQCD [5].

A lot has happened since. While the largest part of the phase diagram is not accessible to experimental measurement or first-principle QCD methods such as Lattice QCD, a few certain statements can be made. For one, as just mentioned, Lattice QCD predicts a crossover along the temperature axis at small densities. On the other hand, it is known that at asymptotically large $\mu$ and moderate $T$, quark matter is a color superconductor $[6,7,8]$. This result is model-independent because at very high densities the QCD coupling is weak due to asymptotic freedom such that rigorous calculations are possible.

Another well-established piece of the QCD phase diagram is found inside the hadronic phase, namely the liquid-gas phase transition for nuclear 


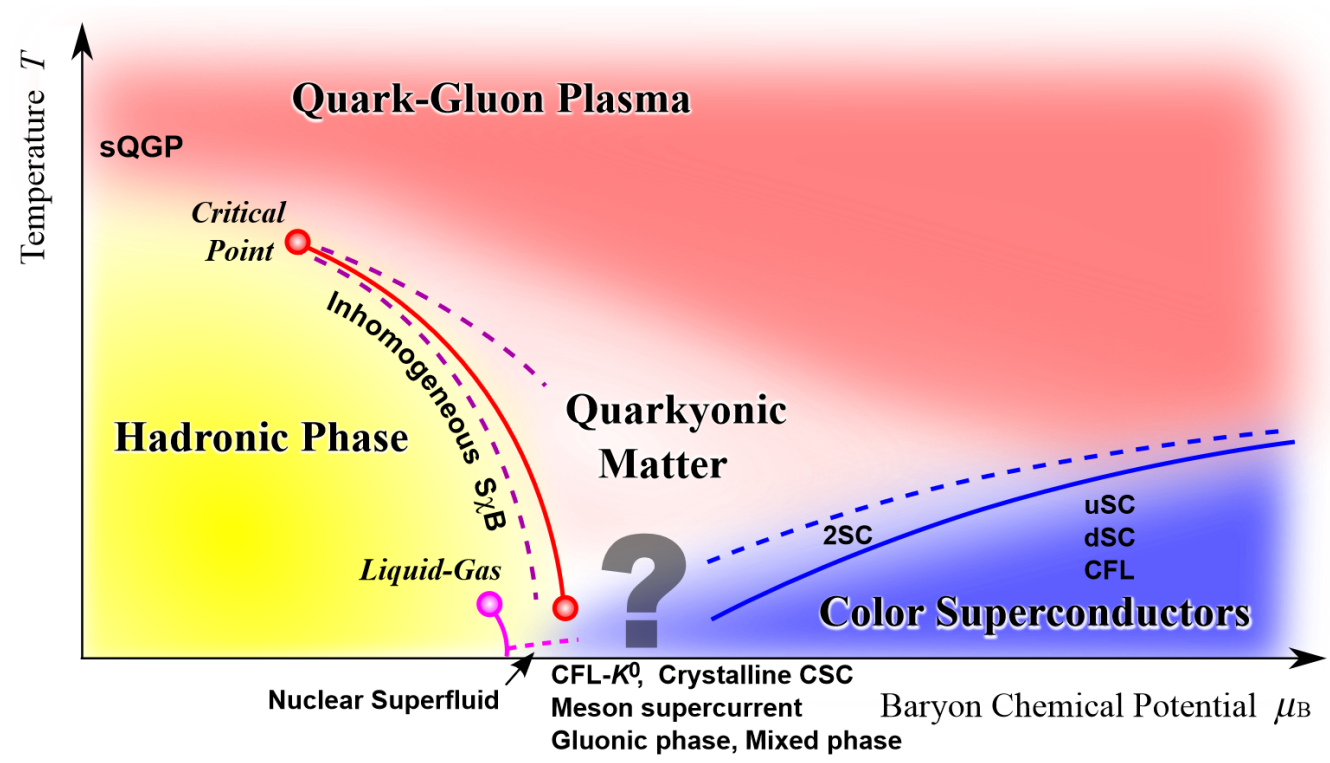

FIGURE 1.2: A QCD phase diagram compiled from knowledge obtained by LQCD (along the temperature axis at vanishing chemical potential), perturbation theory (for very large chemical potential), experimental data (nuclear liquid-gas phase transition), and LEMs (everywhere else). Figure is taken from Ref. [10].

matter, see also Section 5.1.1 in Ref. [9] and refs. therein for an overview of experimental findings. The analyses of many studies show that the liquidgas phase transition is of first order with a second-order critical endpoint. The critical temperature is not precisely determined and lies in the range $T_{c} \sim 5-20 \mathrm{MeV}$.

For the exploration of the remaining pieces of the phase diagram we have no other choice than using effective low-energy models such as the NambuJona-Lasinio (NJL) model. The NJL model is particularly suitable for phase diagram studies of strongly interacting matter since it inherits not only all the symmetries of QCD, but also its most important symmetry-breaking pattern, namely the spontaneous breaking of chiral symmetry. However, confinement can not be described in the NJL model due to missing gluon degrees of freedom. Mean-field calculations of the NJL model show that the chiral symmetry gets restored in a first-order phase transition at large $\mu$ and moderate $T$, which then terminates at a second-order critical endpoint.

If we now compile the results from experiment, lattice studies, and LEMs, we arrive at our currently best guess for the phase diagram of QCD, which is schematically presented in Fig. 1.2.

Of course, the QCD phase diagram can become even more complex, for instance by including another axis for the isospin chemical potential which accounts for the imbalance between up and down quarks in the two-flavor case. For neutron-star studies the consideration of this imbalance is of utmost importance since the electric charge neutrality of neutron stars necessitates a finite isospin chemical potential. Another important example would be the consideration of external magnetic fields. This is not only relevant for neutron stars, in particular magnetars, but also for noncentral heavy-ion 
collisions or for studies of the early Universe [11].

The focus of this thesis lies in the investigation of inhomogeneous chiral phases, which are usually found to supersede the first-order chiral phase transition. Inhomogeneous phases are characterized by an order parameter that varies as a function of spatial coordinates. Inhomogeneous chiral phases correspond in some cases also to a varying quark number density, therefore they are sometimes also called crystal or crystalline phases. As early as the 1960s, inhomogeneous phases were subject of research. For instance, spin density waves have been discussed in condensed-matter physics [12], in particular in the context of superconductivity [13]. One of the first publications on inhomogeneous phases in strong-interaction matter was about density waves in nuclear matter [14] and later on, in the 1970s and 1980s, many studies have been published about inhomogeneous pion condensation $[15,16$, 17].

Since then, inhomogeneous phases have been found in many different QCD-like models. Noteworthy are for example 1+1-dimensional Gross-Neveu (GN) model studies in the large- $N$ limit $[18,19]$ or studies in the linear $\sigma$ model [20], and in the NJL model [21]. In most of these studies, an analytical treatment of inhomogeneous condensates is possible because specific Ansätze for the shape of the chiral order parameter are chosen, such as the chiral density wave (CDW) or the real-kink crystal (RKC) Ansatz. But there are also alternative approaches to inhomogeneous chiral phases, where no specific Ansatz for the shape of the condensate is chosen, e.g., Ginzburg-Landau theory $[22,23]$ and studies where the action of the theory in mean-field approximation is minimized [24].

As seen in Fig. 1.2, the location of the inhomogeneous chiral phase in the phase diagram might be very close to the region where color superconductivity sets in. It is therefore an interesting question, how far such a colorsuperconducting phase extends to lower chemical potential $\mu$ and whether it even overlaps with an inhomogeneous chiral phase. There are only very few studies that address this question [21, 25, 26] and indeed they found a phase where the inhomogeneous chiral condensate and the two-flavor colorsuperconducting phase (2SC) condensate coexist. However, only in Ref. [21] a full phase diagram was calculated and there the Lifshitz and the tricritical point do not coincide, which contradicts the Ginzburg-Landau studies of Ref. [22].

One part of this thesis builds on Ref. [21] by extending it in the following ways: We investigate the phase diagram at finite $T$ and $\mu$ using the PauliVillars regularization instead of the 3D cutoff regularization based on a Taylor expansion of the grand potential in the wave number $q$ as done in Refs. $[21,25]$. The reason for this is that the mismatch of the Lifshitz and the tricritical point is most likely due to the particular regularization scheme used in Ref. [21]. Second, while in Refs. [21, 25, 26] the CDW Ansatz was used, we extend it by the RKC Ansatz in order to investigate how the competition between inhomogeneous chiral phases and two-flavor color superconductivity is affected by the particular shape of the chiral order parameter. 
The second part of this thesis is an investigation of the competition between inhomogeneous chiral phases and two-flavor color superconductivity in the FRG framework. Since almost all studies are done in the mean-field approximation, it is not clear on whether inhomogeneous phases will survive when bosonic fluctuations are included. Instead of using a specific Ansatz for the shape of the chiral order parameter, we perform a stability analysis as first proposed in Ref. [27]. In the quark-meson-model such a stability analysis has been performed first in Ref. [1] where an instability was found in a region of the phase diagram where inhomogeneous chiral phases in meanfield studies are typically found. In this thesis we extend the quark-meson model by diquark degrees of freedom, which we then call the quark-mesondiquark model, and investigate how the instability is affected by the diquark degrees of freedom. 



\section{Chapter 2}

\section{Quantum Chromodynamics and Low-Energy Effective Models}

\subsection{Quantum Chromodynamics}

Quantum Chromodynamics is the fundamental theory of the strong interaction. Its degrees of freedom are the quarks, which are spin- $1 / 2$ fermions and the gluons, which are spin- 1 gauge bosons and mediate the strong force. The quarks come in six different flavors, which in ascending order of mass are named up $(u)$, down $(d)$, strange $(s)$, charm $(c)$, bottom $(b)$, top $(t)$. The masses and electric charges of the quarks are listed in Tab. 2.1. Each of the quarks also carries a color charge, which is the reason for their strongly interacting nature. The quarks can carry three possible color charges, which we will label red, green, blue. The gluons on the other hand do not come in flavors, but they carry both color and anticolor charge. The complex dynamical behavior of quarks and gluons is dictated by the Lagrangian of QCD, which can be separated into three different contributions:

$$
\mathcal{L}_{\mathrm{QCD}}=\mathcal{L}_{\text {Dirac }}+\mathcal{L}_{\mathrm{YM}}+\mathcal{L}_{\mathrm{Ghost}}
$$

The first part describes the dynamics of the quarks and their interaction with the gluons:

$$
\mathcal{L}_{\text {Dirac }}=\bar{\psi}(x)(i \not D-M) \psi(x)
$$

Quark properties

\begin{tabular}{|lllllll|}
\hline Flavor & up (u) & down (d) & strange (s) & charm (c) & bottom (b) & top (t) \\
Mass & $2.16_{-0.26}^{+0.49} \mathrm{MeV}$ & $4.67_{-0.17}^{+0.48} \mathrm{MeV}$ & $93_{-5}^{+11} \mathrm{MeV}$ & $1.27 \pm 0.02 \mathrm{GeV}$ & $4.18_{-0.2}^{+0.3} \mathrm{GeV}$ & $172.76 \pm 0.3 \mathrm{GeV}$ \\
Charge & $2 / 3$ & $-1 / 3$ & $-1 / 3$ & $2 / 3$ & $-1 / 3$ & $2 / 3$ \\
\hline
\end{tabular}

TABLE 2.1: Each quark is a fermion with spin $1 / 2$ and carries a baryon number of $1 / 3$. For each quark there is also an antiquark, which has the same mass and opposite signs for the charge-like quantum numbers. The masses are taken from the latest particle data group (PDG) data [28], which are obtained in the $\overline{\mathrm{MS}}$ scheme. 
where $\psi(x)$ contains all quark fields and is therefore a $4 N_{f} N_{c}$-dimensional spinor. The mass matrix $M$ is a diagonal $4 N_{f} N_{c} \times 4 N_{f} N_{c}$ matrix:

$$
M=\left(\begin{array}{cccc}
m_{\mathrm{up}} \mathbb{1}_{4 N_{c}} & 0 & 0 & \cdots \\
0 & m_{\text {down }} \mathbb{1}_{4 N_{c}} & 0 & \cdots \\
0 & 0 & m_{\text {strange }} \mathbb{1}_{4 N_{c}} & \cdots \\
\vdots & \vdots & \vdots & \ddots
\end{array}\right)
$$

The covariant derivative is defined as

$$
\begin{aligned}
\not D & =\gamma^{\mu} D_{\mu}, \\
D_{\mu} & =\partial_{\mu} \mathbb{1}_{N_{f} N_{c}}-i g A_{\mu}^{a} T^{a} \otimes \mathbb{1}_{N_{f}},
\end{aligned}
$$

where $A_{\mu}^{a}$ are the gluon fields and $T^{a}$ are the $N_{c}^{2}-1$ generators of the nonAbelian $S U\left(N_{c}\right)_{c}$ group in the adjoint representation, which are half the GellMann matrices. There are three different colors in total, therefore the index $a$ runs from $a=1, \ldots, 8$, meaning there are eight different gluons. The strong coupling determines the interaction strength between the quarks and gluons and between the gluons themselves, who are in contrast to photons in Quantum Electrodynamics self-interacting already at tree level ${ }^{1}$. This is described by the Yang-Mills part of QCD:

$$
\mathcal{L}_{\mathrm{YM}}=-\frac{1}{4} F_{\mu \nu}^{a} F^{a, \mu \nu}
$$

where $F_{\mu \nu}^{a}$ is the gluon field strength tensor and defined as

$$
F_{\mu \nu}^{a}=\partial_{\mu} A_{v}^{a}-\partial_{\nu} A_{\mu}^{a}+g f^{a b c} A_{\mu}^{a} A_{v}^{b}
$$

with the antisymmetric structure constants extracted from the relation $\left[T^{a}, T^{b}\right]$ $=i f^{a b c} T^{c}$.

The last part, $\mathcal{L}_{\text {Ghost }}$, has no actual physical content, but is necessary from a technical point of view because it ensures that one does not integrate over gauge-equivalent gauge-field configurations in the path integral formalism. Note that the Lagrangian in Eq. (2.1) is classical, i.e., it does not intrinsically contain quantum effects. Although the QCD Lagrangian describes quantum fields that are defined in spacetime, these are not to be confused with wave functions, which are proper quantum-mechanical waves, whose domain is the configuration space of an $N$ particle system and which as Hilbert-space objects obey the Schrödinger equation. In order to obtain a fully functional quantum-mechanical description of quarks and gluons, in some form or another it is necessary to apply the Schrödinger equation to the fundamental

\footnotetext{
${ }^{1}$ Photons on the other hand do not interact with themselves at tree level, however, loop corrections lead to very small effective self-interaction terms.
} 
degrees of freedom, namely the quantum fields. Nowadays, the most established approach to quantizing QCD is the so-called path-integral formalism, where all possible field configurations are summed up, from which we are then able to derive physical observables.

This approach can be derived from the Schrödinger equation ${ }^{2}$, however in most applications it is more feasible to evaluate the path integral instead of solving the Schrödinger equation. Therefore, in this thesis we will for all practical calculations apply the path-integral formalism.

\section{Asymptotic Freedom and Confinement}

Asymptotic freedom states that the interaction strength decreases for increasing momentum transfer. Since QCD is a non-abelian gauge theory it is also asymptotically free, which allows for a perturbative treatment for large energy scales or small length scales, respectively. Using perturbation theory one can solve the Callan-Symanzik equation for the strong coupling in order to obtain the QCD running coupling, which in leading order yields

$$
\alpha_{\mathrm{s}}(q)=\frac{2 \pi}{\left(\frac{11}{3} N_{\mathrm{C}}-\frac{2}{3} N_{\mathrm{f}}\right) \ln \frac{q}{\Lambda_{\mathrm{QCD}}}}
$$

with $\alpha_{\mathrm{s}}=\frac{g^{2}}{4 \pi}$. The emergence of the QCD scale $\Lambda_{\mathrm{QCD}}$ is a phenomenon called dimensional transmutation. It marks the scale at which the running coupling constant starts to diverge ${ }^{3}$. Below this scale non-perturbative methods must be applied.

Deeply connected to asymptotic freedom is the phenomenon of confinement. Confinement is the phenomenon that quarks and gluons are never observed as isolated particles, as they always clump together to form hadrons with zero net color charge. A low-energy description of QCD must therefore account for confinement by incorporating hadrons as the dominant degrees of freedom.

\section{Chiral Symmetry}

The symmetries of QCD are very important for this thesis, as they need to be realized in low-energy effective models of QCD as well. We list the symmetries of QCD in Tab. 2.2. Chiral symmetry and in particular the breaking thereof will be discussed in more detail here, as it is of particular importance for the understanding of this work. Chiral symmetry is defined as the combined symmetry group $S U\left(N_{f}\right)_{\mathrm{V}} \times S U\left(N_{f}\right)_{\mathrm{A}}$, which is associated with two

\footnotetext{
${ }^{2}$ Or the Schrödinger equation can be derived from the path-integral formalism, depending on your point of view.

${ }^{3}$ Note that the QCD scale depends on the renormalization scheme. For a recent review and estimates of $\Lambda_{\mathrm{QCD}}$, see Ref. [29].
} 
symmetry transformations defined in Tab. 2.2. Let us apply these transformations to $\mathcal{L}_{\text {Dirac }}$ (the Yang-Mills and ghost parts of the QCD Lagrangian are trivially invariant with respect to these transformations):

$$
\begin{aligned}
\mathcal{L}_{\text {Dirac }} \stackrel{S U\left(N_{f}\right)_{\mathrm{V}}}{\rightarrow} \mathcal{L}_{\text {Dirac }}^{\prime} & =\bar{\psi} \mathrm{e}^{-i \vec{\theta}_{\mathrm{V}} \cdot \vec{T}}(i \not D-M) \mathrm{e}^{i \vec{\theta}_{\mathrm{V}} \cdot \vec{T}^{T}} \psi \\
& =\bar{\psi}(i \not D-M) \psi=\mathcal{L}_{\text {Dirac }}, \\
\mathcal{L}_{\text {Dirac }} \stackrel{S U\left(N_{f}\right)_{\mathrm{A}}}{\rightarrow} \mathcal{L}_{\text {Dirac }}^{\prime} & =\bar{\psi} \mathrm{e}^{i \gamma_{5} \vec{\theta}_{\mathrm{A}} \cdot \vec{T}}(i \not D-M) \mathrm{e}^{i \gamma_{5} \vec{\theta}_{\mathrm{A}} \cdot \vec{T}_{T}} \psi \\
& =\bar{\psi}\left(i \not D-\mathrm{e}^{2 i \gamma_{5} \vec{\theta}_{\mathrm{A}} \cdot \vec{T}^{T}} M\right) \psi \neq \mathcal{L}_{\text {Dirac }} .
\end{aligned}
$$

The QCD Lagrangian is evidently invariant under $S U\left(N_{f}\right)_{\mathrm{V}}$ transformations but not under $S U\left(N_{f}\right)_{\text {A }}$ transformations. However, this is not to be confused with the spontaneous breaking, as it is broken explicitly due to a nonvanishing quark mass.

The explicit breaking of this symmetry is better understood when explicitly writing the Lagrangian in terms of left- and right-handed spinors, which represent the two chirality eigenstates of the quark field. To this end, we introduce the projection operator

$$
\mathcal{P}_{\mathrm{R} / \mathrm{L}}=\frac{1 \pm \gamma_{5}}{2}
$$

with the right- and left-handed quark spinors defined as

$$
\psi_{\mathrm{R} / \mathrm{L}}=\mathcal{P}_{\mathrm{R} / \mathrm{L}} \psi
$$

In this basis, the Lagrangian can be rewritten as

$$
\begin{aligned}
\mathcal{L}_{\text {Dirac }} & =\bar{\psi}(i \not D-M) \psi=\bar{\psi}_{\mathrm{R}} i \not D \psi_{\mathrm{R}}+\bar{\psi}_{\mathrm{L}} i \not D \psi_{\mathrm{L}}-\bar{\psi}_{\mathrm{R}} M \psi_{\mathrm{L}}-\bar{\psi}_{\mathrm{L}} M \psi_{\mathrm{R}} \\
& =\left(\begin{array}{c}
\bar{\psi}_{\mathrm{R}} \\
\bar{\psi}_{\mathrm{L}}
\end{array}\right)\left(\begin{array}{cc}
i \not D & -M \\
-M & i \not D
\end{array}\right)\left(\begin{array}{l}
\psi_{\mathrm{R}} \\
\psi_{\mathrm{L}}
\end{array}\right) .
\end{aligned}
$$

It now becomes clear that the mass term mixes the right- and left-handed chirality states, while the kinetic term is diagonal in the new basis. To this end, let us now define an isomorphic symmetry group $S U\left(N_{f}\right)_{\mathrm{R}} \times S U\left(N_{f}\right)_{\mathrm{L}}=$ $S U\left(N_{f}\right)_{\mathrm{V}} \times S U\left(N_{f}\right)_{\mathrm{A}}$ with the associated transformations

$$
\begin{aligned}
& \psi_{\mathrm{R}} \stackrel{\operatorname{SU}\left(N_{f}\right)_{\mathrm{R}}}{\longrightarrow} \mathrm{e}^{i \vec{\theta}_{\mathrm{R}} \cdot \vec{T}_{\mathrm{T}}} \psi_{\mathrm{R}}, \quad \psi_{\mathrm{L}} \stackrel{\operatorname{SU}\left(N_{f}\right)_{\mathrm{L}}}{\longrightarrow} \mathrm{e}^{i \vec{\theta}_{\mathrm{L}} \cdot \vec{T}} \psi_{\mathrm{L}}, \\
& \vec{\theta}_{\mathrm{R}}=\vec{\theta}_{\mathrm{V}}+\vec{\theta}_{\mathrm{A}}, \quad \vec{\theta}_{\mathrm{L}}=\vec{\theta}_{\mathrm{V}}-\vec{\theta}_{\mathrm{A}} \text {. }
\end{aligned}
$$

In this representation, we immediately notice that it is precisely the mixing of the opposite chirality degrees of freedom that breaks the chiral symmetry explicitly. However, in the limit of vanishing quark masses, i.e., $M=0$, chiral 


\section{Symmetries of QCD}

\begin{tabular}{|c|c|c|c|}
\hline Symmetry & Associated Transformation & Type & Broken? \\
\hline Poincaré Symmetry & $\begin{array}{l}A_{\mu}(x) \stackrel{x_{\mu}^{\prime}=\Lambda_{v}^{\mu} x^{v}}{\longrightarrow} \Lambda_{v}^{\mu} A^{v}\left(\Lambda^{-1} x\right), \\
\psi \stackrel{x_{\mu}^{\prime}=\Lambda_{v}^{\mu} x^{v}}{\longrightarrow} S[\Lambda] \psi\left(\Lambda^{-1} x\right)\end{array}$ & spacetime & Exact \\
\hline $\begin{array}{l}\text { Charge Conjugation and } \\
\text { Parity }\end{array}$ & $\psi(t, \vec{x}) \stackrel{P}{\rightarrow} \psi(t,-\vec{x}), \psi \stackrel{C}{\rightarrow} C \bar{\psi}^{\mathrm{T}}$ & Discrete & Exact \\
\hline Time Reversal & $\psi(t, \vec{x}) \stackrel{T}{\rightarrow} \psi(-t, \vec{x})$ & Discrete & $\begin{array}{l}\text { Exact (Equivalent to } \\
\mathrm{CP} \text { symmetry) }\end{array}$ \\
\hline $\begin{array}{l}\text { Baryon Number Symmetry } \\
U(1)_{V}\end{array}$ & $\psi \stackrel{U(1) \mathrm{V}}{\rightarrow} e^{i \theta_{\mathrm{V}}} \psi$ & Global & Exact \\
\hline Axial Symmetry $U(1)_{A}$ & $\psi \stackrel{U(1)}{\rightarrow}{ }^{i \gamma_{5} \theta_{\mathrm{A}}} \psi$ & Global & $\begin{array}{l}\text { Explicitly broken by } \\
\text { quark masses and } \\
\text { anomalously broken }\end{array}$ \\
\hline Vector Symmetry $S U\left(N_{f}\right)_{\mathrm{V}}$ & $\psi \stackrel{S U\left(N_{f}\right)_{\mathrm{V}}}{\longrightarrow} e^{i \vec{\theta}_{V} \cdot \vec{T}} \psi$ & Global & $\begin{array}{l}\text { Explicitly broken by } \\
\text { non-degenerate mass } \\
\text { matrix }\end{array}$ \\
\hline $\begin{array}{l}\text { Axial-Vector } \quad \text { Symmetry } \\
\operatorname{SU}\left(N_{f}\right)_{\mathrm{A}}\end{array}$ & $\psi \stackrel{\operatorname{SU}\left(N_{f}\right)_{\mathrm{A}}}{\longrightarrow} e^{i \vec{\theta}_{V} \cdot \vec{T} \gamma_{5}} \psi$ & Global & $\begin{array}{lr}\text { Explicitly } & \text { broken } \\
\text { by non-zero quark } \\
\text { masses }\end{array}$ \\
\hline Local Color $S U\left(N_{C}\right)$ & $\begin{array}{l}A_{\mu} \stackrel{S U\left(N_{c}\right)}{\longrightarrow} U_{c}(x) A_{\mu} U_{c}^{\dagger}(x)- \\
\frac{i}{g}\left(\partial_{\mu} U_{c}(x)\right) U_{c}^{\dagger}(x) \text { and } \psi \stackrel{S U\left(N_{c}\right)}{\longrightarrow} \\
U_{c}(x) \psi\end{array}$ & Local & Exact \\
\hline Dilatation Symmetry & $\begin{array}{l}x_{\mu}^{\prime} \longrightarrow \lambda^{-1} x_{\mu} \text { such that } \\
A_{\mu}^{\prime}\left(x^{\prime}\right) \longrightarrow \lambda A_{\mu}(x)\end{array}$ & Global & $\begin{array}{l}\text { Realized in YM and } \\
\text { in Dirac sector for } \\
\text { small quark masses, } \\
\text { anomalously broken }\end{array}$ \\
\hline
\end{tabular}

TABLE 2.2: An overview of all symmetries of QCD. The transformation matrix for Dirac spinors is defined as $S[\Lambda]=\exp \left(1 / 2 \Omega_{\alpha \beta} S^{\alpha \beta}\right)$, where $\Omega_{\alpha \beta}$ are the generators of the Poincaré group and $S^{\alpha \beta}=-i / 4\left[\gamma_{\alpha}, \gamma_{\beta}\right]$.

symmetry is realized. If we now consider only two flavors, i.e., an up and a down quark, we observe that they are significantly smaller than the QCD scale, $\Lambda_{\mathrm{QCD}} \sim 300 \mathrm{MeV}$, and from a phenomenological point of view, we can therefore start with a chirally symmetric Lagrangian and treat the finite quark masses as a small perturbation around the chirally symmetric limit.

Now that we considered only two quark flavors and can therefore assume chiral symmetry to be approximately realized, let us ponder about the implications. If chiral symmetry for two-flavor QCD described the real world, then we would expect this symmetry to be found among the observed strongly interacting particles, the hadrons. But this symmetry is not found in experiments - hadrons do not come in opposite-parity pairs with approximately equal masses. Therefore, chiral symmetry must be broken even in the zero quark-mass limit, and the resolution to this is spontaneous symmetry breaking.

The mechanism behind the spontaneous breakdown of chiral symmetry can be explained as follows: The production of quark-antiquark pairs comes basically at no cost of energy because, as already elaborated, up and down quarks can be considered almost massless. Particles constantly interact with 
these quark-antiquark pairs, because they are figuratively "omnipresent". Because of the non-perturbative nature of QCD this sea of quark-antiquarks leads to a non-vanishing condensate. Angular-momentum conservation then leads to a condensate of the form

$$
\langle\bar{q} q\rangle=\left\langle 0\left|q_{\mathrm{L}}^{\dagger} q_{\mathrm{R}}+q_{\mathrm{R}}^{\dagger} q_{\mathrm{L}}\right| 0\right\rangle,
$$

because the right- and left-handed components of quark spinors have opposite chirality. This is the chiral condensate and also the order parameter of the chiral phase transition. Note that the chiral condensate corresponds to a mixing of the right- and left-handed chirality states as shown in Eq. (2.15). Now since quarks strongly interact with the chiral condensate, they get effectively heavier, which breaks chiral symmetry spontaneously. It is now evident why chiral symmetry and its breaking is so important for low-energy models of QCD: It explains the largest proportion of the origin of hadron masses. Furthermore the Goldstone theorem [30] states that the spontaneous breaking of a continuous symmetry leads to $\operatorname{dim}(G)-\operatorname{dim}(H)$ spinless particles with zero mass, where $G$ is the symmetry group of the classical Lagrangian and $H$ the symmetry group of the ground state. In the case of QCD we have chiral symmetry that is spontaneously broken and it generates for two-flavor QCD $\operatorname{dim}\left(S U(2)_{\mathrm{V}} \times S U(2)_{\mathrm{A}}\right)-\operatorname{dim}\left(S U(2)_{\mathrm{V}}\right)=3$ Goldstone bosons which are the pions. Pions are the lightest mesons and therefore they play a crucial role in the low-energy regime of QCD.

\section{Color Superconductivity}

In Ref. [6] it was found that quark matter at large chemical potential and low temperature is a color superconductor, which is characterized by a degenerate Fermi gas of quarks interacting with a condensate of Cooper pairs near the Fermi surface.

This can be proven rigorously because at high densities the QCD coupling is weak due to asymptotic freedom. The rationale is as follows: Due to the weak QCD coupling at large densities we can neglect interactions, i.e., the quarks near the Fermi surface are almost free. Thus the energy required to add a particle to the system is just the Fermi energy $E_{\mathrm{F}}=\mu$ and therefore adding quarks or antiquarks costs no free energy near the Fermi surface:

$$
\Omega(N)=E-\mu N \longrightarrow \Omega(N+1)=(E+\mu)-\mu(N+1)=\Omega(N) .
$$

Now, if we have a weak attractive channel between a pair of quarks or antiquarks, the free energy is even lowered by the amount of the potential energy of their attraction:

$$
\Omega(N)=E-\mu N \longrightarrow \Omega(N+2)=\left(E+2 \mu-E_{\mathrm{B}}\right)-\mu(N+2)=\Omega(N)-E_{\mathrm{B}}
$$


Therefore, more stability is achieved by adding bound states at the Fermi surface. We certainly know that in some channels the quark-quark interaction must be attractive, since we know that quarks form baryons. In App. B we prove this for a two-flavor quark-quark channel with total angular momentum $J=0$.

The attractive force leads to a formation of bound states, which are the Cooper pairs of color superconductivity ${ }^{4}$. From a macroscopic viewpoint Cooper pairs are composite bosons obeying Bose-Einstein statistics and thus forming a condensate. This condensate in turn then affects the dispersion relations of the quarks as they interact with the condensate:

$$
\omega_{ \pm}=\sqrt{\left(E_{\vec{p}} \pm \mu\right)^{2}+|\Delta|^{2}},
$$

where $E_{\vec{p}}=\sqrt{\vec{p}^{2}+m^{2}}$ is the quark energy with mass $m$ and $|\Delta|$ the energy gap. This leads to an energy gap of $2|\Delta|$ between the ground state and the lowest excited state and in the case of electromagnetic superconductivity this is the reason why the electric resistance becomes zero: At sufficiently small temperatures all electrons remain in the ground state in the form of Cooper pairs, i.e., the electric current is transported by Cooper pairs, not single electrons. However, Cooper pairs are incapable to interact with other particles: They cannot scatter in such a way that they lose energy, because they are part of the condensate, the lowest-energy state. Furthermore, because of the lowtemperature surrounding, they cannot go into the next-higher excited state through scattering processes ${ }^{5}$.

Back to quark matter, the phenomenology of color superconductivity is not as straightforward, because there are different types of color superconductivity, such as three-flavor color-flavor-locked superconductivity (CFL) and two-flavor color superconductivity (2SC), which have different symmetry breaking patterns leading to different phenomenological consequences.

In this work we will focus on the 2SC phase, which is obtained in the limit of an infinitely large strange quark mass. As shown in App. B we have a scalar color-antitriplet diquark condensate in the 2SC phase:

$$
\left\langle\Delta_{A}\right\rangle=\left\langle\bar{\psi}_{c} i \gamma_{5} \tau_{2} \lambda_{A} \psi\right\rangle,
$$

with the indices $A=2,5,7$ corresponding to the anti-symmetric Gell-Mann matrices. This is a three-dimensional vector in color space, however the condensation happens in one particular direction, which can be chosen arbitrarily. Here we use $A=2$ to define the diquark condensate

\footnotetext{
${ }^{4}$ They are not bound in the sense that its constituents are located close to another, but rather in the sense that they have non-zero correlations between them.

${ }^{5} \mathrm{As}$ absolute zero temperature can never be achieved, there is always a vanishingly small chance that a Cooper pair can scatter.
} 


$$
\Delta \equiv\left\langle\bar{\psi}_{c} i \gamma_{5} \tau_{2} \lambda_{2} \psi\right\rangle
$$

which in our convention means that red $\mathrm{u}$ and green $\mathrm{d}$ quarks are paired, while the blue quarks do not participate in the condensate. This again leads to symmetry breaking in the Andersen-Higgs mechanism. While QCD is $S U(3)_{\mathrm{c}}$ symmetric, the condensate leads to a violation of the local color symmetry because it is only $S U(2)_{c}$ symmetric. This in turn would lead to $\operatorname{dim}\left(S U(3)_{c}\right)-\operatorname{dim}\left(S U(2)_{c}\right)=8-3=5$ Goldstone bosons, however in gauge theories this does not lead to Goldstone bosons, instead, loosely speaking, the Goldstone modes are "eaten up" by the gauge bosons, which then become massive. There are then as many massive gauge bosons as there would have been Goldstone bosons. The remaining three massless gluons correspond to the residual local $S U(2)_{c}$ symmetry. Note that while Eq. 2.23 breaks $S U(3)_{c}$, it is still invariant under chiral symmetry.

The motivation for the investigation of the 2SC phase in this work is as follows: While at very large chemical potentials we expect a CFL phase, at intermediate densities the strange quark can be considered frozen due to its larger mass compared to the up and down quarks. We are in particular interested in the critical region of the phase diagram, i.e., the region of the firstorder chiral phase transition (which is usually superseded by an inhomogeneous chiral phase, when a spatial modulation of the chiral order parameter is considered). In NJL model studies this first-order transition for $T=0$ is typically found at a quark chemical potential $\sim 300 \mathrm{MeV}$, which is somewhat lower than the constituent strange quark mass $m_{s} \sim 500 \mathrm{MeV}$. We therefore assume that the onset of a color-superconducting phase is defined by a $2 \mathrm{SC}$ diquark condensate. ${ }^{6}$

\subsection{Low-Energy Models of QCD}

Most regions of the QCD phase diagram are not accessible with perturbation theory because of asymptotic freedom or lattice methods due to the sign problem, therefore we need to approach low-energy QCD with another strategy, namely by using (effective) low-energy models (LEMs). The goal of LEMs is to find a suitable field theory that incorporates the relevant degrees of freedoms and features of a specific energy scale, while neglecting more complicated dynamics originating from different scales. This not only simplifies the calculations, but in many cases the calculation of some observables is made possible by this approach. Ideally, the neglected dynamics should be irrelevant to the phenomenology that is studied, but unfortunately in case of low-energy QCD this cannot be guaranteed due to its non-perturbative nature.

\footnotetext{
${ }^{6}$ Note however that in compact stars this line of reasoning is not valid anymore because a large volume of quark matter, such as neutron stars, must be electrically neutral and a color singlet [31].
} 


\section{Effective Field Theories/Low-Energy Models}

\begin{tabular}{|c|c|c|}
\hline Model & Summary & Refs. \\
\hline MIT bag model & $\begin{array}{l}\text { Purely phenomenological model in which } \\
\text { quarks are confined inside hadrons. Inside the } \\
\text { hadron they behave as free particles. The bag } \\
\text { model predicts hadron spectrum surprisingly } \\
\text { well except for the pion mass. }\end{array}$ & [32] \\
\hline Skyrmion models & $\begin{array}{l}\text { The Skyrmion is a topologically stable field } \\
\text { configuration and in the Skyrmion models the } \\
\text { baryon number is regarded as a topological } \\
\text { quantum number. This model has only two pa- } \\
\text { rameters to fix and all other low-energy prop- } \\
\text { erties are then predictions. }\end{array}$ & [33] \\
\hline Nambu-Jona-Lasinio model & $\begin{array}{l}\text { Models } \chi \mathrm{SB} \text {, and is non-renormalizable. Con- } \\
\text { tains only quarks and can be seen as "proper" } \\
\text { effective theory since the four-fermi interaction } \\
\text { can be in principle derived from QCD. }\end{array}$ & {$[34,35]$} \\
\hline Quark-Meson (Diquark) Model & $\begin{array}{l}\text { Models } \chi \mathrm{SB} \text {, and is renormalizable. Quarks } \\
\text { and mesons are coupled via Yukawa interac- } \\
\text { tion and exhibits } O(N) \text { symmetry for bosons. }\end{array}$ & [36] \\
\hline Linear Sigma Model & $\begin{array}{l}\text { Models } \chi \mathrm{SB} \text {, contains pions and the sigma me- } \\
\text { son in } O(4) \text { vectors, and baryons are coupled } \\
\text { to the bosons via Yukawa interaction. If the } \\
\text { baryon is interpreted as quark, then it is the } \\
\text { same as the quark-meson model. If the Yukawa } \\
\text { term is dropped it can be generalized to an } \\
O(N) \text { model. }\end{array}$ & {$[37,38]$} \\
\hline Extended Linear Sigma Model & $\begin{array}{l}\text { Similar to the linear sigma model, mesons } \\
\text { are arranged in chiral multiplets and exhibits } \\
\text { spontaneous chiral symmetry breaking; Ex- } \\
\text { otic mesons can also be incorporated, such as } \\
\text { tetraquarks, meson molecules and glueballs; } \\
\text { easy to calculate observables such as masses } \\
\text { and decay widths. }\end{array}$ & $\begin{array}{l}{[39,40,41,} \\
42]\end{array}$ \\
\hline Parity-Doublet Model & $\begin{array}{l}\text { two nucleons are incorporated as chiral part- } \\
\text { ners, often used to study chiral symmetry } \\
\text { restoration in nuclear matter and neutron-star } \\
\text { physics. }\end{array}$ & {$[42,43,44]$} \\
\hline $\begin{array}{ll}\text { Chiral Perturbation } & \text { Theory } \\
(\mathrm{ChPT}) & \end{array}$ & $\begin{array}{l}\text { Based on a non-linear realization of chiral sym- } \\
\text { metry. Can be treated using perturbation the- } \\
\text { ory in terms of an expansion in pion energies. }\end{array}$ & [45] \\
\hline Polyakov-Loop models & $\begin{array}{l}\text { The Polyakov loop can be added in many effec- } \\
\text { tive models of QCD mentioned here. The ex- } \\
\text { pectation value of the Polyakov loop serves as } \\
\text { order parameter for the deconfinement phase. }\end{array}$ & {$[46,47]$} \\
\hline Walecka model & $\begin{array}{l}\text { Also known as } \sigma-\omega \text { model. Commonly used } \\
\text { to calculate the liquid-gas phase transition of } \\
\text { nuclear matter. }\end{array}$ & {$[48,49]$} \\
\hline Vector-Dominance Models & $\begin{array}{l}\text { The electrically neutral light vector mesons } \\
\text { fields such as } \rho_{\mu}^{0}, \omega_{\mu} \text {, and } \phi \text { mesons are identi- } \\
\text { fied with the electromagnetic hadronic current. } \\
\text { This assumption is based on the observation } \\
\text { that hadron interactions with the electroweak } \\
\text { sector happen dominantly via exchange of vec- } \\
\text { tor mesons. }\end{array}$ & [50] \\
\hline
\end{tabular}

TABLE 2.3: A few different low-energy effective models of QCD. Some are of more phenomenological nature, while others, such as ChPT can be understood as proper effective field theories in the sense that they can be treated perturbatively. 
In order to avoid a misunderstanding what we mean by low-energy models, let us compare it with a very similar term, namely effective field theory (EFT). According to standard effective field theory reviews as in Refs. [51, $52,53]$, an appropriate field theory at the respective energy regime should be addressing three questions:

1. What are the relevant degrees of freedom (fields)?

2. What are the symmetries? What interactions result from them and what is the symmetry-breaking pattern?

3. Power counting and matching: What are the expansion parameters of the respective EFT? How to match an EFT to its underlying field theory?

In this work, the first two questions will be our guide to writing down our effective models. The third question, however, is more subtle and therefore we would like to clarify this. If we for example call a quark-meson model an effective field theory with respect to QCD, then this could be considered as misnomer because we actually will not integrate out gluons and quarks and we will not match the emerging operators to the operators of the quark-meson model. What is rather meant in this work when talking about low-energy effective theories of QCD is that we think of a phenomenological model that shares the same symmetries and symmetry-breaking pattern with full QCD. Furthermore, in many effective field-theory lectures a large separation of scales between the effective and the more fundamental underlying theory is assumed, which is most likely not the case for low-energy effective theories of QCD. In order to try to prevent confusion and to highlight that the models used in this work have a more phenomenological nature, we call them low-energy models (LEMs) of QCD instead of effective field theories. This can be further emphasized by the fact that in contemporary high-energy physics there are many different possible models that are used at roughly the same energy scale, and each of them has a slightly different phenomenology. Therefore one can see these models as different representations of low-energy QCD.

In Tab. 2.3 we list some LEMs of QCD. In this work the focus lies on the study of the NJL model and the quark-meson model.

\subsection{The Nambu-Jona-Lasinio Model}

The Nambu-Jona-Lasinio (NJL) model has been developed in Refs. [54, 55] dating back to 1961, a time where quarks were still unknown, and thus QCD as well. However, at that time there were already hints for chiral symmetry breaking, namely due to the experimental fact that the SU(3) pseudoscalarisovector mesons (the pions, kaons, and the eta meson) are significantly lighter than the mesons with different quantum numbers. As discussed in the last section, spontaneous chiral symmetry breaking leads to the appearance of massless Goldstone bosons, which are just the SU(3) pseudoscalar-isovector 
mesons. In addition, the interaction of all other hadrons with the chiral condensate generates the largest part of their masses.

The Lagrangian of the NJL model for $N_{f}=2$ quark flavors in its original form reads

$$
\mathcal{L}_{\mathrm{NJL}}=\bar{\psi}(i \not \partial-m) \psi+G\left[(\bar{\psi} \psi)^{2}+\left(\bar{\psi} i \gamma_{5} \vec{\tau} \psi\right)^{2}\right] .
$$

The NJL Lagrangian consists of the typical Dirac Lagrangian and a fourfermion interaction term with coupling $G$. The four-fermion interaction is symmetric with respect to the $S U(2)_{\mathrm{V}} \times S U(2)_{\mathrm{A}} \times U(1)_{\mathrm{V}}$, whereas the mass $m$ explicitly breaks $S U(2)_{\text {A }}$ symmetry.

Originally the fermions of the NJL model were interpreted as nucleons in order to model nucleon-nucleon interactions, however, after the discovery of QCD it was reinterpreted as a quark model to model low-energy QCD (for reviews, see Refs. [56, 57]). It is easily seen that the NJL model is not a suitable fundamental theory as the four-fermion coupling $G$ has the dimension of an inverse energy squared and therefore it is manifestly nonrenormalizable. Its success as a LEM is of course not a coincidence. For one it has the same global symmetries as QCD, leading to similar phenomenology. And while the point-like four-fermion interaction is not shared with the QCD Lagrangian, it can be seen as an emergent interaction that is obtained when integrating out the gluons. However, as an LEM, it also has its shortcomings. For one, since it is non-renormalizable, the results obtained from the NJL model depend on the specific regularization scheme. This drawback can be ameliorated by using several regularization schemes and comparing how much the results depend on the choice of the specific regularization scheme. Another shortcoming of the NJL model is that the local self-interaction term is not confining and therefore one should apply the NJL model only where confinement is not essential. Since the mechanism of confinement is not yet unraveled, the range of applicability for the NJL model is unknown. This is also connected to the discussion of the last section, where we explained that it is somewhat misleading to speak of effective field theories because there is no clear way how to derive the NJL model from QCD and it should therefore be rather seen as a phenomenological model.

In this work we will use the NJL model and extend it by a diquark interaction term in order to calculate the phase diagram in the mean-field approximation:

$$
\mathcal{L}_{\mathrm{NJL}+\Delta}=\mathcal{L}_{\mathrm{NJL}}+\mathcal{L}_{\Delta}
$$

where the second term in Eq. (2.25) is added in order to describe diquark condensation in the spin-zero color-antitriplet channel,

$$
\mathcal{L}_{\Delta}=G_{\Delta}\left(\bar{\psi}_{c} i \gamma_{5} \tau_{2} \lambda_{A} \psi\right)\left(\bar{\psi} i \gamma_{5} \tau_{2} \lambda_{A} \psi_{c}\right) \text {. }
$$

where $\psi_{c}=C \bar{\psi}^{\mathrm{T}}$, with $C=i \gamma^{2} \gamma^{0}$ being the charge-conjugation matrix, and $\lambda_{A}, A=2,5,7$, are the antisymmetric Gell-Mann matrices in color space. We 
will return to this model in Chapter 3, but now we would like to introduce another low-energy model of QCD, the quark-meson model, which has the same physical content but a different Lagrangian.

\subsection{The Quark-Meson Model}

The Lagrangian of the quark-meson model reads:

$$
\begin{aligned}
\mathcal{L}_{\mathrm{QM}}= & \bar{\psi}\left[i \not \partial-h_{\phi}\left(\sigma+i \gamma_{5} \vec{\tau} \cdot \vec{\pi}\right)\right] \psi \\
& +\frac{1}{2}\left[\left(\partial_{\mu} \sigma\right)^{2}+\left(\partial_{\mu} \vec{\pi}\right)^{2}\right]-U\left(\phi^{2}\right)+c \sigma .
\end{aligned}
$$

The quark-meson model is symmetric with respect to $S U(2)_{\mathrm{V}} \times S U(2)_{\mathrm{A}} \times$ $\mathrm{U}(1)_{\mathrm{V}}$, except for the explicit symmetry breaking term, $c \sigma$, which breaks the $S U(2)_{\text {A }}$ symmetry:

$$
\sigma \stackrel{S U(2)}{\longrightarrow} \sigma^{\prime}=\sigma \mathrm{e}^{i \gamma_{5} \vec{\theta}_{A} \cdot \vec{\tau}}
$$

In general, the potential $U\left(\phi^{2}, \Delta^{2}\right)$ includes all terms that are allowed by chiral symmetry and therefore it can be written as

$$
U\left(\phi^{2}\right)=\sum_{n=0}^{\infty} \lambda_{n}\left(\sigma^{2}+\vec{\pi}^{2}\right)^{n}
$$

As in the case for the NJL model, the major reason to study the QM model is the simple fact that it mimics the symmetries of QCD while being far simpler due to the fact that gluonic degrees of freedom are missing. Compared to the NJL model it also has the advantage that it contains both quarks and mesons as dynamic degrees of freedoms and it is renormalizable, and therefore it is especially suitable for FRG studies [36, 47, 58, 59, 60].

The relation between the NJL model and the QM model is rarely discussed in literature and it is often not clear why one would choose one over the other. In particular, it is somewhat peculiar, why the QM model is renormalizable while the NJL model is not, despite the fact that both models have the same physical content and the same symmetries. Here we would like to have a closer look on the relation between these two models by showing how one can arrive at one model starting from the other.

To this end, we consider the chiral limit, i.e., $m=0$, and we follow Ref. [61]. First, we perform a Hubbard-Stratonovich transformation on the fourpoint part of the NJL Lagrangian, introducing the fields $\sigma$ and $\vec{\pi}$ : 


$$
\begin{aligned}
& \exp \left\{i \int_{x}\left[(\bar{\psi} \psi)^{2}+\left(\bar{\psi} i \gamma_{5} \vec{\tau} \psi\right)^{2}\right]\right\}= \\
& \mathcal{N} \int \mathcal{D} \sigma \mathcal{D} \vec{\pi}\left\{i \int_{x}\left[-\frac{\left(\sigma^{2}+\vec{\pi}^{2}\right)}{4 G}+\bar{\psi}\left(\sigma+i \gamma_{5} \vec{\tau} \cdot \vec{\pi}\right) \psi\right]\right\} .
\end{aligned}
$$

The generating functional for the NJL model is then found to be

$$
\mathcal{Z}=\int \mathcal{D} \sigma \mathcal{D} \vec{\pi} \mathcal{D} \bar{\psi} \mathcal{D} \psi \mathrm{e}^{i \int_{x} \tilde{\mathcal{L}}_{\mathrm{NJL}}}
$$

with the new Lagrangian

$$
\tilde{\mathcal{L}}_{\mathrm{NJL}}=\bar{\psi}\left[i \not \partial-h_{\phi} \sigma_{0}+h_{\phi}\left(\sigma+i \gamma_{5} \vec{\tau} \cdot \vec{\pi}\right)\right] \psi-h_{\phi}^{2} \frac{\left(\sigma^{2}+\vec{\pi}^{2}\right)}{4 G}
$$

where we introduced the Yukawa coupling $h_{\phi}$ by redefining $\sigma \rightarrow h_{\phi} \sigma$ and $\vec{\pi} \rightarrow h_{\phi} \vec{\pi}$, and shifted $\sigma \rightarrow \sigma+\sigma_{0}$. Next, we integrate over the fermionic degrees of freedom:

$$
\mathcal{Z}=\int \mathcal{D} \sigma \mathcal{D} \vec{\pi} \exp \left\{i \operatorname{Tr} \ln \left[i \not \partial-h_{\phi} \sigma_{0}-h_{\phi}\left(\sigma+i \gamma_{5} \vec{\tau} \cdot \vec{\pi}\right)\right]-i \int_{x} h_{\phi}^{2} \frac{\left(\sigma^{2}+\vec{\pi}^{2}\right)}{4 G}\right\}
$$

Let us now focus on the $\operatorname{Tr} \ln$ term:

$$
\begin{aligned}
\operatorname{Tr} & \ln \left[i \not \partial-h_{\phi} \sigma_{0}-h_{\phi}\left(\sigma+i \gamma_{5} \vec{\tau} \cdot \vec{\pi}\right)\right] \\
& =\operatorname{Tr} \ln \left[\left(i \not \partial-h_{\phi} \sigma_{0}\right)\left(1-\frac{1}{i \not \partial-h_{\phi} \sigma_{0}} h_{\phi}\left(\sigma+i \gamma_{5} \vec{\tau} \cdot \vec{\pi}\right)\right)\right] \\
& =\operatorname{Tr} \ln \left[i \not \partial-h_{\phi} \sigma_{0}\right]+\operatorname{Tr} \ln \left[1-\frac{1}{i \not \partial-h_{\phi} \sigma_{0}} h_{\phi}\left(\sigma+i \gamma_{5} \vec{\tau} \cdot \vec{\pi}\right)\right] .
\end{aligned}
$$

The first term is just an irrelevant constant. The second term can now be expanded in a log power series:

$$
\begin{aligned}
i \operatorname{Tr} \ln \left[1-\frac{1}{i \not \partial-h_{\phi} \sigma_{0}} h_{\phi}\left(\sigma+i \gamma_{5} \vec{\tau} \cdot \vec{\pi}\right)\right] & =-\sum_{n=1}^{\infty} \frac{i}{n} \operatorname{Tr}\left[\frac{1}{i \not \partial-h_{\phi} \sigma_{0}} h_{\phi}\left(\sigma+i \gamma_{5} \vec{\tau} \cdot \vec{\pi}\right)\right]^{n} \\
& =-\sum_{n=1}^{\infty} \frac{i}{n} \operatorname{Tr}\left[\frac{\left(-i \not \partial-h_{\phi} \sigma_{0}\right)}{\partial_{\mu} \partial^{\mu}+\left(h_{\phi} \sigma_{0}\right)^{2}} h_{\phi}\left(\sigma+i \gamma_{5} \vec{\tau} \cdot \vec{\pi}\right)\right]^{n} \\
& \equiv-\sum_{n=1}^{\infty} U^{(n)}
\end{aligned}
$$



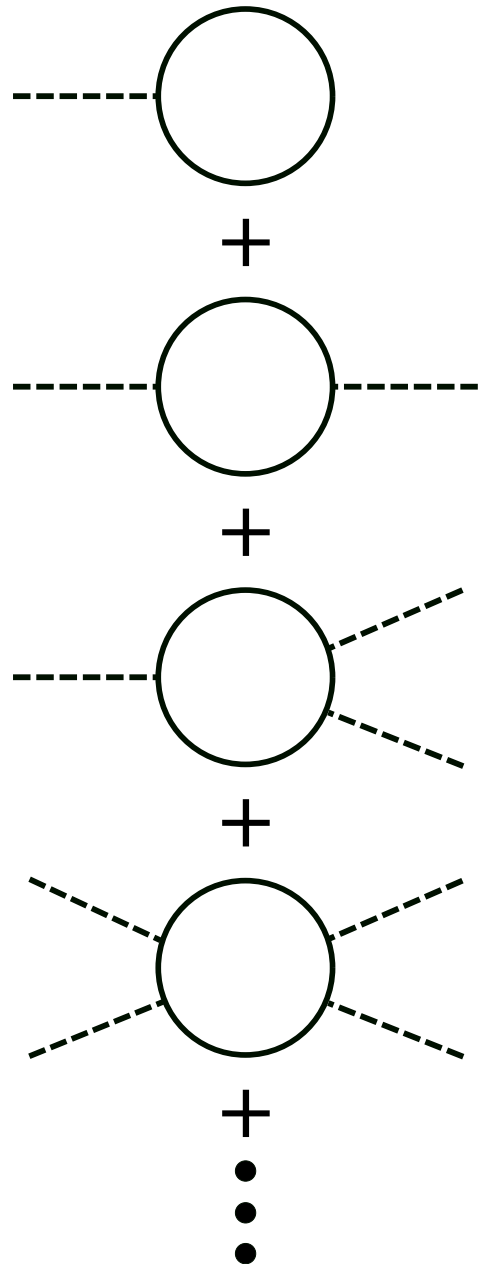

FIGURE 2.1: Diagrammatic representation of the $\mathrm{Tr} \ln$ expansion in Eq. (2.35). The solid lines are the fermion loops, while the dashed lines correspond to the $\sigma$ or the $\vec{\pi}$ mesons, respectively.
The lowest four orders, $n \leq 4$, yield divergent diagrams that need to be renormalized, which yield quadratically and logarithmically divergent loop integrals. Explicitly, these are

$$
\begin{aligned}
I_{\log } & =-4 i \int_{q} \frac{1}{\left[q^{2}-\left(h_{\phi} \sigma_{0}\right)^{2}\right]^{2}}, \\
I_{\text {quad }} & =4 i \int_{q} \frac{1}{q^{2}-\left(h_{\phi} \sigma_{0}\right)^{2}} .
\end{aligned}
$$

The derivation is shown in Refs. [61, 62] and derived with more detail for the two-color NJL model in Refs. [63, 64]. Furthermore, we define

$$
\mathcal{L}_{\mathrm{conv}}=\sum_{n=2}^{4} U_{c}^{(n)}+\sum_{n=5}^{\infty} U^{(n)},
$$

where $U_{c}^{(n)}$ denotes the convergent part of $U^{(n)}$, while $U^{(n)}, n>5$, are fully convergent. Finally, we arrive at the following effective Lagrangian:

$$
\begin{aligned}
\mathcal{L}_{\text {eff }} & =\frac{1}{2} I_{\log } h_{\phi}^{2}\left[\left(\partial_{\mu} \sigma\right)^{2}+\left(\partial_{\mu} \vec{\pi}\right)^{2}\right] \\
& -\frac{h_{\phi}^{2}}{2}\left[\frac{1}{2 G}-2 I_{\text {quad }}-I_{\log } 2\left(h_{\phi} \sigma_{0}\right)^{2}\right]\left(\sigma^{2}+\vec{\pi}^{2}\right) \\
& -\frac{h_{\phi}^{4}}{2} I_{\log }\left(\sigma^{2}+\vec{\pi}^{2}\right)^{2}+\mathcal{L}_{\text {conv }}
\end{aligned}
$$

Here, we have obtained kinetic and interaction terms by including radiative corrections. Note, that if we now apply a proper regularization for the loop integrals and redefine the couplings, the new effective Lagrangian would look like a normal renormalizable Lagrangian. Indeed, if we introduce renormalized fields and couplings, 


$$
\begin{aligned}
\sigma_{\mathrm{R}} & =\frac{\sigma}{\sqrt{Z}}, \quad \vec{\pi}_{\mathrm{R}}=\frac{\vec{\pi}}{\sqrt{Z}}, \\
I_{\log } h_{\phi}^{2} & =\frac{1}{Z}, \\
I_{\log } h_{\phi}^{4} & =\frac{\lambda_{0}}{2 Z_{\lambda}}, \\
I_{\text {quad }} & =\frac{1}{4 G}, \\
\lambda & =\frac{\lambda_{0} Z^{2}}{Z \lambda}, \\
\sigma_{0}^{2} & =-\frac{Z M^{2}}{2 \lambda} .
\end{aligned}
$$

the effective Lagrangian can then be reformulated in a more familiar form :

$$
\mathcal{L}_{\text {eff }}=\frac{1}{2}\left[\left(\partial_{\mu} \sigma_{\mathrm{R}}\right)^{2}+\left(\partial_{\mu} \vec{\pi}_{\mathrm{R}}\right)^{2}\right]-\frac{M^{2}}{2}\left(\sigma_{\mathrm{R}}^{2}+\vec{\pi}_{\mathrm{R}}^{2}\right)-\frac{\lambda}{4}\left(\sigma_{\mathrm{R}}^{2}+\vec{\pi}_{\mathrm{R}}^{2}\right)^{2}+\mathcal{L}_{\mathrm{conv}} .
$$

Neglecting the convergent contribution $\mathcal{L}_{\text {conv }}$ this is just the Lagrangian of the linear sigma model.

Now, let us perform a similar analysis for the quark-meson model defined in Eq. (2.27). First, we note that the explicit symmetry breaking term $c \sigma$ is equivalent to a fermion mass term $\bar{\psi} m_{q} \psi$. To show this simply perform a shift, $\sigma \rightarrow \sigma-\frac{m_{q}}{h_{\phi}}$, and choose the explicit symmetry-breaking parameter, $c$, such that the linear terms in $U_{k}\left(\left(\sigma-m_{q} / h_{\phi}\right)^{2}+\vec{\pi}^{2}\right)$ vanish. For instance, if the effective potential is simply $U_{k}\left(\phi^{2}\right)=m^{2} / 2\left(\sigma^{2}+\vec{\pi}^{2}\right)$, then upon shifting the $\sigma$ field we obtain

$$
c=m^{2} \frac{m_{q}}{h_{\phi}}
$$

Note however, that this definition of the explicit symmetry-breaking parameter is modified as more interaction terms in the effective potential are considered. Again, we consider the chiral limit, i.e., $m_{q}=c=0$, and shift $\sigma \rightarrow \sigma+\sigma_{0}$. Now, if we integrate out the fermionic fluctuations for the QM model and expand the Tr $\ln$ in the same way as we did for the bosonized NJL model, we arrive at the following effective Lagrangian [61]: 


$$
\begin{aligned}
\mathcal{L}_{\mathrm{QM}, \mathrm{eff}}= & -\frac{1}{2}\left[m^{2}-2 I_{\text {quad }} h_{\phi}^{2}-2 I_{\log } h_{\phi}^{2}\left(h_{\phi} \sigma_{0}\right)^{2}\right]\left(\sigma^{2}+\vec{\pi}^{2}\right) \\
& +\frac{1}{2}\left(1+I_{\log } h_{\phi}^{2}\right)\left[\left(\partial_{\mu} \sigma\right)^{2}+\left(\partial_{\mu} \vec{\pi}\right)^{2}\right]-\frac{1}{4}\left(\lambda+2 I_{\log } h_{\phi}^{4}\right)\left(\sigma^{2}+\vec{\pi}^{2}\right)^{2} \\
& +\tilde{U}\left(\phi^{2}\right)+\mathcal{L}_{\mathrm{conv}} .
\end{aligned}
$$

We explicitly wrote out the mass and self-interaction terms of the potential:

$$
U\left(\phi^{2}\right) \equiv \frac{m^{2}}{2}\left(\sigma^{2}+\vec{\pi}^{2}\right)+\frac{\lambda}{4}\left(\sigma^{2}+\vec{\pi}^{2}\right)^{2}+\tilde{U}\left(\phi^{2}\right)
$$

where $\tilde{U}\left(\phi^{2}\right)$ contains all higher-order self-interaction terms.

Now we define the renormalization parameters

$$
\begin{aligned}
\sigma_{\mathrm{R}} & =\frac{\sigma}{\sqrt{Z}}, \quad \vec{\pi}_{\mathrm{R}}=\frac{\vec{\pi}}{\sqrt{Z}}, \\
1+I_{\log } h_{\phi}^{2} & =\frac{1}{Z} \\
\lambda+2 I_{\log } h_{\phi}^{4} & =\frac{\lambda_{0}}{Z_{\lambda}}, \\
m^{2}+\lambda \sigma_{0}^{2}-2 I_{\text {quad }} h_{\phi}^{2} & =0, \\
\sigma_{0}^{2} & =-\frac{Z M^{\prime 2}}{2 \lambda^{\prime}}, \\
\lambda^{\prime} & =\frac{\lambda_{0} Z^{2}}{Z_{\lambda}} .
\end{aligned}
$$

$\mathcal{L}_{\text {conv }}$ contains all the terms that are also in $\tilde{U}\left(\phi^{2}\right)$, therefore we will omit $\tilde{U}\left(\phi^{2}\right)$ in the final step. The new Lagrangian in terms of the renormalized fields reads

$$
\begin{aligned}
\mathcal{L}_{\mathrm{QM}, \text { eff }}= & \frac{1}{2}\left[\left(\partial_{\mu} \sigma_{\mathrm{R}}\right)^{2}+\left(\partial_{\mu} \vec{\pi}_{\mathrm{R}}\right)^{2}\right]-\frac{1}{2} M^{\prime 2}\left(\sigma_{\mathrm{R}}^{2}+\vec{\pi}_{\mathrm{R}}^{2}\right) \\
& -\frac{1}{4} \lambda^{\prime}\left(\sigma_{\mathrm{R}}^{2}+\vec{\pi}_{\mathrm{R}}^{2}\right)^{2}+\mathcal{L}_{\text {conv }} .
\end{aligned}
$$

The new effective Lagrangian is again just a linear sigma-model Lagrangian when neglecting $\mathcal{L}_{\text {conv }}$.

This analysis makes it clear what the relation between the NJL model and the QM model actually is. Both models are just different starting UV actions, which upon integrating out fermionic fluctuations lead to the same linear sigma model-type effective action. However, while for the NJL model the kinetic terms are generated by radiative corrections, they are already included in the QM model. Thus the NJL model could be seen as more fundamental 
than the QM model. Furthermore, the reason that the QM model is renormalizable but the NJL not becomes now evident: In the QM model the divergent parts are assumed to be renormalized and absorbed in the couplings of the QM model.

In FRG studies, the main reason to use the quark-meson model instead of a bosonised NJL model is for the practical reason that the kinetic term $\partial_{\mu} \phi \partial^{\mu} \phi$ yields a $\vec{p}^{2}$ term in the Wetterich equation, which then simply gets canceled when using the optimized Litim regulator, $R_{k}=\left(k^{2}-\vec{p}^{2}\right) \theta\left(k^{2}-\vec{p}^{2}\right)$, simplifying the flow equation for the effective potential significantly. This will become more clear in Sec. 4 where we will discuss the FRG formalism in more detail.

\subsection{The Quark-Meson-Diquark Model}

One of the main topics of this thesis is the study of the quark-meson-diquark model for three-color QCD with the functional renormalization group. The Lagrangian of this model reads

$$
\begin{aligned}
& \mathcal{L}_{\mathrm{QMD}}=\bar{\psi}\left[i \not \partial-\mu \gamma_{0}+h_{\phi}\left(\sigma+i \gamma_{5} \vec{\tau} \cdot \vec{\pi}\right)\right] \psi \\
& +\frac{h_{\Delta}}{2}\left[\Delta^{* a} \psi^{\mathrm{T}} C \tau_{2} \epsilon^{a} \gamma_{5} \psi-\Delta^{a} \bar{\psi} \gamma_{5} \epsilon^{a} \tau_{2} C \bar{\psi}^{\mathrm{T}}\right]+\frac{1}{2}\left[\left(\partial_{\mu} \sigma\right)^{2}+\left(\partial_{\mu} \vec{\pi}\right)^{2}\right] \\
& +\frac{1}{2}\left[\left(\partial_{\nu}-2 \delta_{\nu 0} \mu\right) \Delta^{* a}\right]\left[\left(\partial_{v}+2 \delta_{\nu 0} \mu\right) \Delta^{a}\right]+U\left(\phi^{2},\left|\Delta_{a}\right|^{2}\right)-c \sigma,
\end{aligned}
$$

which can be seen as a quark-meson model extended by a diquark-quark interaction term and a kinetic term for the diquarks ${ }^{7}$. The first line is essentially the same as in the QM model, however, here we have included the quark chemical potential term $\bar{\psi} \mu \gamma_{0} \psi$. The first term in the second line models the quark-diquark interaction. Here again we have the kinetic term for the sigma and pion fields, but also for the complex diquark fields. Since the diquark is a complex scalar field the zero components of the usual kinetic term is modified $\partial_{0} \rightarrow \partial_{0} \pm \mu_{\Delta}$, where $\mu_{\Delta}=2 \mu$, because the diquark is composed of two quarks. The potential term is similar to the one in the QM model but with the diquark invariants considered:

$$
U\left(\phi^{2},|\Delta|^{2}\right)=\sum_{n=0, m=0}^{\infty}\left[\lambda_{n, m}\left(\sigma^{2}+\vec{\pi}^{2}\right)^{n}\left(\Delta_{a} \Delta_{a}^{*}\right)^{m}\right] .
$$

To our knowledge, Ref. [65] is the only FRG study of the quark-mesondiquark model as an effective low-energy model for three-color QCD. The

\footnotetext{
${ }^{7}$ As a remark, here we can again apply the discussion of the last section where we compared the QM and the NJL model: The kinetic terms can be obtained from radiative corrections when starting from Eq. (2.25). One can see the QMD model and the NJL model with diquarks as different truncations of the same theory, which both lead to a linear sigma-model Lagrangian extended by diquarks when integrating out the quark degrees of freedom.
} 
reason we highlight "three-color" is the fact, that the quark-meson-diquark model is also known as a low-energy model for two-color QCD, see also Refs. [65, 66, 67, 68, 69, 70]. But from now on we will call the three-color QMD model just QMD model. The two-color variant of this model can be obtained by replacing $\Delta_{a} \rightarrow \Delta$, where $\Delta$ in two-color QCD also happens to be a baryon. Furthermore, we need to identify $h_{\Delta} \equiv h_{\phi}$ in order to restore the $S O(6)$ symmetry of $\mathrm{QC}_{2} \mathrm{D}$ in vacuum [71, 72]. The main reason to study a model for two-color QCD instead of a physical QCD model is because of the absence of the sign problem in $\mathrm{QC}_{2} \mathrm{D}$, enabling lattice studies of the $\mathrm{QC}_{2} \mathrm{D}$ phase diagram $[73,74]$. The lattice data can then be used as benchmark for the FRG study of the QMD. Note that in this thesis we will only study the three-color variant. 


\section{Chapter 3}

\section{Competition of Inhomogeneous Chiral Condensates with Color Superconductivity in the Mean-Field Approximation}

This chapter is based on Ref. [75] and here we will calculate the phase diagram for the NJL model with diquarks in the mean-field approximation (MFA). The MFA is also known as Hartree-Fock approximation and there is a rich literature on it with applications not only in high-energy physics, but also atomic physics, condensed-matter physics, and chemistry.

The MFA has also a long history in research on non-perturbative lowenergy models, and although it is a rather rough approximation, it yields physically reasonable results surprisingly well. However, there might be a confirmation bias at play. Since so many studies in non-perturbative highenergy physics have been performed in the MFA, these results seem to be the norm and what we expect. Therefore it is important to go beyond the MFA using the FRG, which will be discussed later in this thesis.

The starting point is the NJL Lagrangian Eq. (2.25). Furthermore, we consider quark-number fluctuations by adding the quark chemical potential:

$$
\begin{aligned}
\mathcal{L}_{\mathrm{NJL}+\Delta}= & \bar{\psi}\left(i \gamma^{\mu} \partial_{\mu}+\mu \gamma_{0}\right) \psi+G\left[(\bar{\psi} \psi)^{2}+\left(\bar{\psi} i \gamma_{5} \vec{\tau} \psi\right)^{2}\right] \\
& +G_{\Delta}\left(\bar{\psi}_{c} i \gamma_{5} \tau_{2} \lambda_{a} \psi\right)\left(\bar{\psi} i \gamma_{5} \tau_{2} \lambda_{a} \psi_{c}\right) .
\end{aligned}
$$

A common implementation of the MFA is achieved by linearizing the Lagrangian with respect to its fermion bilinears. This is done by introducing the expectation values

$$
\langle\bar{\psi} \psi\rangle=\sigma, \quad\left\langle\bar{\psi} i \gamma_{5} \vec{\tau} \psi\right\rangle=\vec{\pi}, \quad\left\langle\bar{\psi}_{c} i \gamma_{5} \tau_{2} \lambda_{a} \psi\right\rangle=\Delta_{a},
$$

and thus

$$
\bar{\psi} \psi=\sigma+\delta \sigma, \quad \bar{\psi} i \gamma_{5} \vec{\tau} \psi=\vec{\pi}+\delta \vec{\pi}, \quad \bar{\psi}_{c} i \gamma_{5} \tau_{2} \lambda_{a} \psi=\Delta_{a}+\delta \Delta_{a} .
$$


This results in a Lagrangian quadratic in fluctuations such that fermionic fluctuations can be integrated out analytically.

Here we use an alternative approach that yields the same results. The action of the model is bosonized by performing a Hubbard-Stratonovich transformation and the fermion degrees of freedom are doubled in such a way that it leaves the total path integral unchanged [76], see App. C.1 for the detailed calculation. After bosonizing the action we obtain the following effective Lagrangian

$$
\begin{aligned}
\mathcal{L}_{\text {eff }}= & \frac{1}{2}\left[\bar{\psi}\left(i \not \partial+\mu \gamma_{0}+\sigma+i \gamma_{5} \vec{\pi} \cdot \vec{\tau}\right) \psi+\bar{\psi}_{c}\left(i \not \partial-\mu \gamma_{0}+\sigma+i \gamma_{5} \vec{\pi} \cdot \vec{\tau}\right) \psi_{c}\right. \\
& \left.+\Delta_{a}\left(\bar{\psi}_{c} i \gamma_{5} \tau^{2} \lambda^{a} \psi\right)+\Delta_{a}^{*}\left(\bar{\psi} i \gamma_{5} \tau^{2} \lambda^{a} \psi_{c}\right)-\frac{\sigma^{2}+\vec{\pi}^{2}}{2 G}-\frac{\left|\Delta_{a}\right|^{2}}{2 G_{\Delta}}\right] \\
\equiv & \frac{1}{2}\left(\bar{\Psi} \mathcal{S}^{-1} \Psi-\frac{\sigma^{2}+\vec{\pi}^{2}}{2 G}-\frac{\left|\Delta_{a}\right|^{2}}{2 G_{\Delta}}\right)
\end{aligned}
$$

where the real auxiliary fields $\sigma$ and $\vec{\pi}$ correspond to scalar- and pseudoscalarmesonic degrees of freedom, and the complex auxiliary fields $\Delta_{A}$ correspond to diquarks. In the last step we introduced Nambu-Gor'kov spinors $\Psi=$ $\left(\psi, \psi_{c}\right)^{\mathrm{T}}$ for a more compact notation and defined the inverse propagator

$$
\mathcal{S}^{-1}=\left(\begin{array}{cc}
i \not \partial-\hat{M}+\mu \gamma^{0} & \hat{\Delta} \\
-\hat{\Delta}^{+} & i \not \partial-\hat{M}-\mu \gamma_{0}
\end{array}\right),
$$

with

$$
\hat{M}=-\sigma-i \gamma_{5} \vec{\tau} \cdot \vec{\pi}
$$

and

$$
\hat{\Delta}=i \gamma_{5} \tau_{2} \lambda_{a} \Delta_{a}
$$

\subsection{Grand Potential}

For spatially varying scalar and pseudoscalar fields $\sigma(\mathbf{x}), \vec{\pi}(\mathbf{x})$ and a constant color-superconducting gap parameter $\Delta_{a}=\delta_{a 2} \Delta$, the grand potential reads in MFA

$$
\Omega=-\frac{T}{V} \ln \mathcal{Z}=\Omega_{\text {kin }}+\frac{1}{4 G} \frac{1}{V} \int d^{3} \mathbf{x}\left[\sigma^{2}(\mathbf{x})+\vec{\pi}^{2}(\mathbf{x})\right]+\frac{|\Delta|^{2}}{4 G_{\Delta}},
$$

where

$$
\Omega_{\text {kin }} \equiv-\frac{1}{2} \frac{T}{V} \operatorname{Tr} \ln \left(\frac{\mathcal{S}^{-1}}{T}\right)
$$

$\mathcal{Z}$ is the generating functional for the Lagrangian of Eq. (3.4). The trace is taken over space-time, spin, color, and flavor indices. The bosonic part does not come with a Tr ln term because we have neglected bosonic fluctuations. 
Now, instead of specifying an Ansatz for $\sigma^{2}(\mathbf{x})$ and $\vec{\pi}^{2}(\mathbf{x})$ we will rewrite the generating functional as an integral over the spectrum of the full Hamiltonian of the theory. We will then see that the resulting density of states only contains the spectrum of the theory without diquarks. This is a desirable result since this allows us to use density of states that are already known from NJL model studies without diquarks.

To this end we follow the approach of Refs. $[77,78]$ and isolate the time derivative

$$
\mathcal{S}^{-1}=\gamma^{0}\left(i \partial_{0}-H_{\mathrm{NG}}\right),
$$

with the effective Dirac Hamiltonian

$$
H_{\mathrm{NG}}=\left(\begin{array}{cc}
H-\mu & -\gamma^{0} \hat{\Delta} \\
\gamma^{0} \hat{\Delta}^{+} & H+\mu
\end{array}\right)
$$

where

$$
H \equiv-i \gamma^{0} \vec{\not}+\gamma^{0} \hat{M}
$$

is the effective Hamiltonian in the case without diquark pairing. Equation (3.9) can then be written as

$$
\Omega_{\mathrm{kin}}=-\frac{T}{2 V} \sum_{n} \sum_{\lambda} \ln \left(\frac{i \omega_{n}+\mathcal{E}_{\lambda}}{T}\right),
$$

where $\mathcal{E}_{\lambda}$ are the eigenvalues of $H_{\mathrm{NG}}$.

The Matsubara sum can be evaluated with standard techniques [79] and yields

$$
T \sum_{n} \ln \left(\frac{i \omega_{n}+\mathcal{E}_{\lambda}}{T}\right)=\frac{\left|\mathcal{E}_{\lambda}\right|}{2}+T \ln \left(1+\mathrm{e}^{-\left|\mathcal{E}_{\lambda}\right| / T}\right)
$$

We determine the eigenvalues $\mathcal{E}_{\lambda}$ by squaring $H_{\mathrm{NG}}$, which yields the blockdiagonal matrix (C.3)

$$
H_{\mathrm{NG}}^{2}=\left(\begin{array}{cc}
(H-\mu)^{2}+|\Delta|^{2} \mathcal{P}_{r g} & 0 \\
0 & (H+\mu)^{2}+|\Delta|^{2} \mathcal{P}_{r g}
\end{array}\right),
$$

where

$$
\mathcal{P}_{\text {rg }}=\lambda_{2}^{2}=\left(\begin{array}{lll}
1 & 0 & 0 \\
0 & 1 & 0 \\
0 & 0 & 0
\end{array}\right)
$$

is identified as the projector onto the space of gapped quark colors.

The eigenvalues can now be read off from those of the squared Hamiltonian, which are $\left(E_{\lambda} \mp \mu\right)^{2}$ for the ungapped quark/antiquark and, with twofold degeneracy, $\left(E_{\lambda} \mp \mu\right)^{2}+|\Delta|^{2}$ for the gapped quarks/antiquarks, where $E_{\lambda}$ are the eigenvalues of $H$. The absolute values $\epsilon_{\lambda, \pm} \equiv\left|\mathcal{E}_{\lambda, \pm}\right|$ for quarks / 
antiquarks are thus found to be

$$
\begin{array}{ll}
\epsilon_{\lambda, \pm}=\sqrt{\left(E_{\lambda} \mp \mu\right)^{2}+|\Delta|^{2}} & \text { for the gapped quarks, } \\
\epsilon_{\lambda, \pm}^{(0)}=\left|E_{\lambda} \mp \mu\right| & \text { for the ungapped quark. }
\end{array}
$$

Inserting these into Eq.(3.13) yields

$$
\begin{array}{r}
\Omega_{\text {kin }}=-\frac{1}{2} \sum_{i= \pm} \frac{1}{V} \sum_{\lambda}\left[\epsilon_{\lambda, i}+2 T \ln \left(1+\mathrm{e}^{-\epsilon_{\lambda, i} / T}\right)\right. \\
\left.+\frac{\epsilon_{\lambda, i}^{(0)}}{2}+T \ln \left(1+\mathrm{e}^{-\epsilon_{\lambda, i}^{(0)} / T}\right)\right] .
\end{array}
$$

Now we can define the density of states for $H$ as

$$
\rho(E)=\frac{1}{V} \sum_{\lambda} \delta\left(E-E_{\lambda}\right),
$$

which allows us to express Eq. (3.19) in the following way:

$$
\begin{array}{r}
\Omega_{\text {kin }}=-\frac{1}{2} \sum_{i= \pm} \int_{-\infty}^{\infty} d E \rho(E)\left[\epsilon_{i}+2 T \ln \left(1+\mathrm{e}^{-\epsilon_{i} / T}\right)\right. \\
\left.+\frac{\epsilon_{i}^{(0)}}{2}+T \ln \left(1+\mathrm{e}^{-\epsilon_{i}^{(0)} / T}\right)\right],
\end{array}
$$

with

$$
\epsilon_{ \pm}=\sqrt{(E \mp \mu)^{2}+|\Delta|^{2}} \quad \text { and } \quad \epsilon_{ \pm}^{(0)}=|E \mp \mu| .
$$

Since the original spectrum and, as a consequence, $\rho(E)$ are symmetric around zero, we can replace $\frac{1}{2} \int_{-\infty}^{\infty} d E$ by $\int_{0}^{\infty} d E$. Our final result for the grand potential is then given by

$$
\begin{aligned}
\Omega= & -\sum_{i= \pm} \int_{0}^{\infty} d E \rho(E)\left[\epsilon_{i}+2 T \ln \left(1+\mathrm{e}^{-\epsilon_{i} / T}\right)+\frac{\epsilon_{i}^{(0)}}{2}+T \ln \left(1+\mathrm{e}^{-\epsilon_{i}^{(0)} / T}\right)\right] \\
& +\frac{1}{4 G} \frac{1}{V} \int d^{3} \mathbf{x}\left[\sigma^{2}(\mathbf{x})+\vec{\pi}^{2}(\mathbf{x})\right]+\frac{|\Delta|^{2}}{4 G_{\Delta}} .
\end{aligned}
$$

Note that $\rho(E)$ was defined by the spectrum of $H$, the Hamiltonian in absence of diquark pairing. Thus, the diquarks contribute to the grand potential only through the energies $\epsilon_{ \pm}$. This means that if $\rho(E)$ is known in the absence of diquark condensation, the extension to include homogeneous 2SC condensates is straightforward.

Incidentally, if we take the limit $\Delta \rightarrow 0$ of Eq. (3.23) we obtain 


$$
\begin{aligned}
\Omega_{\Delta \rightarrow 0} & =-6 \int_{0}^{\infty} d E \rho(E)\left[E+T \ln \left(1+\mathrm{e}^{-(E-\mu) / T}\right)+T \ln \left(1+\mathrm{e}^{-(E+\mu) / T}\right)\right] \\
& +\frac{1}{4 G} \frac{1}{V} \int d^{3} \mathbf{x}\left[\sigma^{2}(\mathbf{x})+\vec{\pi}^{2}(\mathbf{x})\right]
\end{aligned}
$$

which is exactly the grand potential of the NJL model without diquarks, see also Ref. [78]. We therefore expect to exactly reproduce the results for the standard NJL Lagrangian in this limit.

\subsubsection{Inhomogeneous Chiral Condensates}

We now evaluate the grand potential (3.23) for the CDW and RKC configurations. The respective densities of states have been found in Ref. [77]. It is already known that, without diquark condensation, the RKC solution is preferred over the CDW one, and we do not expect that this will change when accounting for diquark condensation. Nevertheless, here we study both Ansätze, in order to confirm this expectation.

\section{Chiral Density Wave}

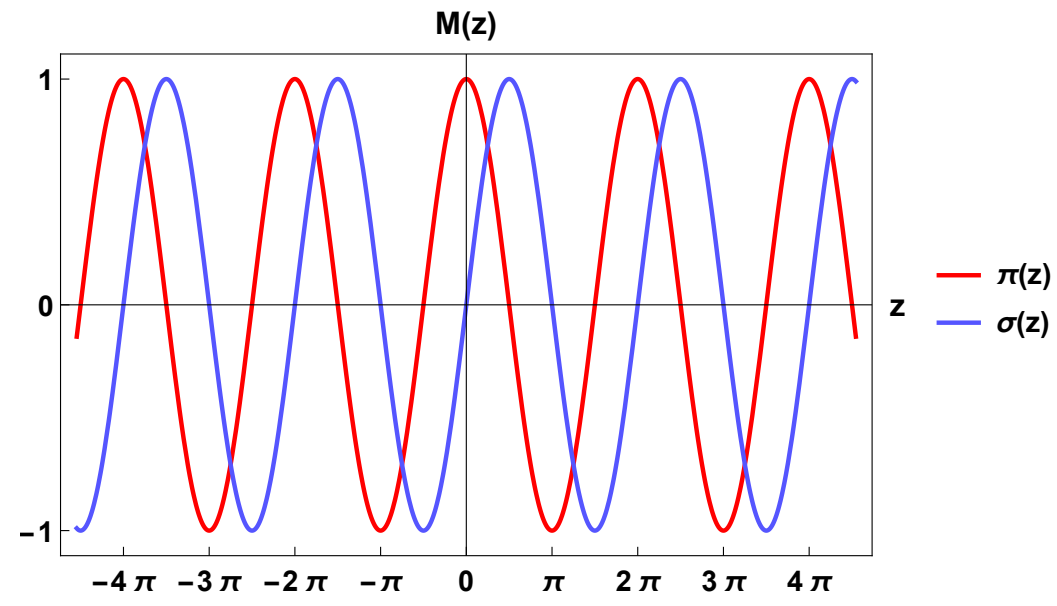

FIGURE 3.1: The CDW for $q=1$ and amplitude $M=1$.

For the one-dimensional CDW the Ansatz for the inhomogeneous chiral condensate is given by

$$
M(z)=\sigma(z)+i \gamma_{5} \vec{\pi}(z) \cdot \vec{\tau}=-M \mathrm{e}^{i \gamma_{5} \tau_{3} q z},
$$

with an amplitude $M$ and the wave number $q$ of the CDW. Without loss of generality, we have chosen the one-dimensional modulation to align with the $z$-axis. In the case $q=0, M$ is equal to the constituent quark mass.

The eigenvalues $E_{\lambda}$ for the CDW without a color-superconducting condensate have been determined in Refs. [80, 81]. Inserting these into Eqs. 
(3.17), (3.18) yields the respective eigenvalues in the presence of a colorsuperconducting condensate, which are identical to those found in Ref. [25].

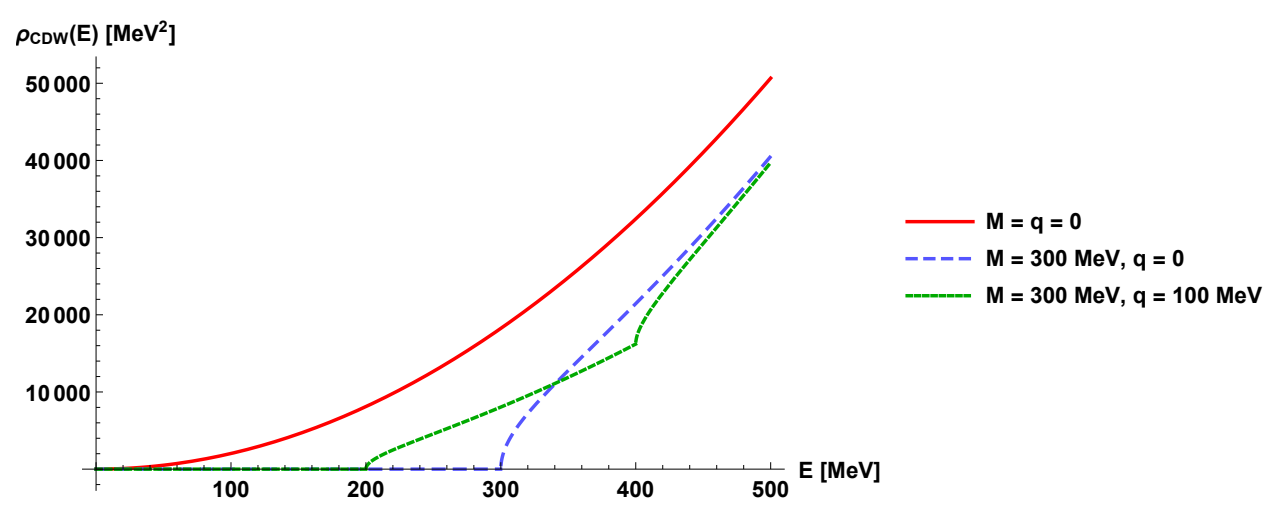

FIGURE 3.2: The density of states for the CDW Ansatz. For the case $M=q=0$, which corresponds to the restored phase, the quarks are not bound hadrons and therefore the density of states increases continuously as more energy is added to the system. The case $M=300 \mathrm{MeV}$ and $q=0$ corresponds to the chirally broken phase and here we nicely see that the density of states is zero for energies smaller than the gap $M$. At energies larger than the gap, the hadrons dissolve because the quarks overcome the attractive potential that binds them. For non-vanishing wave number the density of states sets in already at $E=M-q$ because quarks can scatter off the inhomogeneous condensate.

The density of states (3.20) for the CDW has been found in Ref. [77],

$$
\begin{aligned}
\rho_{\mathrm{CDW}}(E)=\frac{N_{f} E}{2 \pi^{2}}[ & \theta(E-q-M) \sqrt{(E-q)^{2}-M^{2}} \\
& +\theta(E-q+M) \theta(E+q-M) \sqrt{(E+q)^{2}-M^{2}} \\
& \left.+\theta(q-M-E)\left(\sqrt{(E+q)^{2}-M^{2}}-\sqrt{(E-q)^{2}-M^{2}}\right)\right] .
\end{aligned}
$$

In Fig. 3.2 we present a plot of the CDW density of states for different parameters, which makes Eq. (3.26) more intuitive.

Inserting the density of states into Eq. (3.23) we find

$$
\begin{aligned}
\Omega_{\mathrm{CDW}} & =-\sum_{i= \pm} \int_{0}^{\infty} d E \rho_{\mathrm{CDW}}(E)\left[\epsilon_{i}+\frac{\epsilon_{i}^{(0)}}{2}\right. \\
& \left.+2 T \ln \left(1+\mathrm{e}^{-\epsilon_{i} / T}\right)+T \ln \left(1+\mathrm{e}^{-\epsilon_{i}^{(0)} / T}\right)\right] \\
& +\frac{M^{2}}{4 G}+\frac{|\Delta|^{2}}{4 G_{\Delta}} .
\end{aligned}
$$




\section{Real-Kink Crystal}

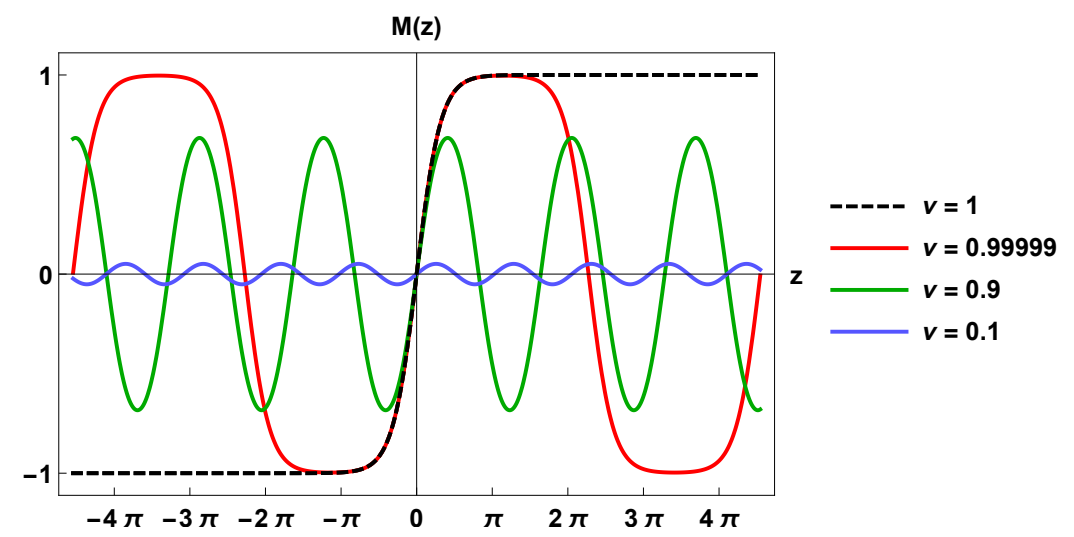

FIGURE 3.3: The RKC for various $v$ and $D=1$. For $v=1$ the RKC Ansatz becomes $M(z)=D \tanh D z$.

For the one-dimensional RKC, the pion field is set to zero and the Ansatz for the sigma field reads

$$
\sigma(z) \equiv v D \frac{\operatorname{sn}(D z \mid v) \operatorname{cn}(D z \mid v)}{\operatorname{dn}(D z \mid v)}
$$

where sn, $\mathrm{cn}$, dn are Jacobi elliptic functions. The parameter $v$ determines the shape of the condensate: For $v \rightarrow 1$ the Ansatz becomes $D \tanh (D z)$, i.e., a kink-like soliton of amplitude $D$ and width $1 / D$. For $v \rightarrow 0$ it becomes a sine of infinitesimal amplitude.

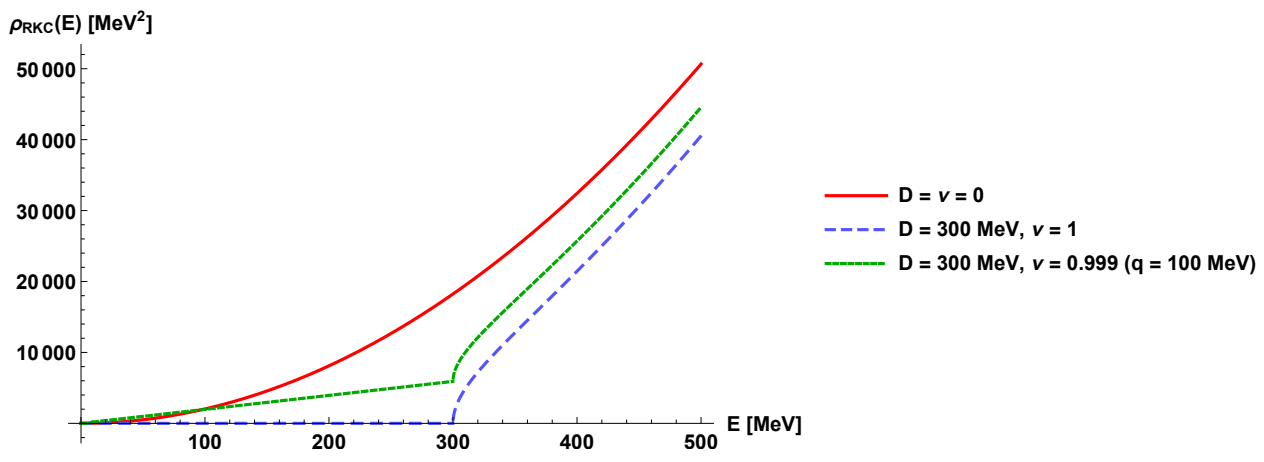

FIGURE 3.4: In contrast to the CDW Ansatz, we have for non-vanishing wave number a non-zero density of states even for smaller energies than $E=M-q$. This shows that the notion of a wave number is misleading for the RKC because the RKC solution can be written as a Fourier series with infinitely many non-vanishing Fourier coefficients.

The density of states for the RKC has already been computed in Ref. [77], 


$$
\begin{aligned}
\rho_{\mathrm{RKC}}(E) & =\frac{N_{f} E D}{\pi^{2}}\left\{\theta(\sqrt{\tilde{v}} D-E)\left[\mathbf{E}(\tilde{\theta} \mid \tilde{v})+\left(\frac{\mathbf{E}(v)}{\mathbf{K}(v)}-1\right) \mathbf{F}(\tilde{\theta} \mid \tilde{v})\right]\right. \\
& +\theta(E-\sqrt{\tilde{v}} D) \theta(D-E)\left[\mathbf{E}(\tilde{v})+\left(\frac{\mathbf{E}(v)}{\mathbf{K}(v)}-1\right) \mathbf{K}(\tilde{v})\right] \\
& \left.+\theta(E-D)\left[\mathbf{E}(\theta \mid \tilde{v})+\left(\frac{\mathbf{E}(v)}{\mathbf{K}(v)}-1\right) \mathbf{F}(\theta \mid \tilde{v})+\frac{\sqrt{\left(E^{2}-D^{2}\right)\left(E^{2}-\tilde{v} D^{2}\right)}}{E D}\right]\right\},
\end{aligned}
$$

where $\mathbf{K}(\cdot)$ are the complete and $\mathbf{F}(\cdot \mid \cdot)$ the incomplete elliptic integrals of the first kind, respectively, while $\mathrm{E}(\cdot)$ are the complete and $\mathrm{E}(\cdot \mid \cdot)$ the incomplete elliptic integrals of the second kind. We followed the notational convention of Ref. [77], where $\tilde{v}=1-v, \tilde{\theta}=\arcsin [E /(\sqrt{\tilde{v}} D)]$, and $\theta=\arcsin (D / E)$. The grand potential for the RKC Ansatz is found to be

$$
\begin{aligned}
\Omega_{\mathrm{RKC}} & =-\sum_{i= \pm} \int_{0}^{\infty} d E \rho_{\mathrm{RKC}}(E)\left[\epsilon_{i}+\frac{\epsilon_{i}^{(0)}}{2}\right. \\
& \left.+2 T \ln \left(1+\mathrm{e}^{-\epsilon_{i} / T}\right)+T \ln \left(1+\mathrm{e}^{-\epsilon_{i}^{(0)} / T}\right)\right] \\
& +\frac{M^{2}}{4 G}+\frac{|\Delta|^{2}}{4 G_{\Delta}},
\end{aligned}
$$

where we introduced the average squared amplitude of the RKC,

$$
M^{2} \equiv \frac{1}{L} \int_{0}^{L} d z|\sigma(z)|^{2}
$$

with the period $L \equiv 4 \mathbf{K}(v) / D$ of the RKC. For later purposes, we also define the effective wave number of the RKC as $q \equiv 2 \pi / L$. In Fig. 3.4 we provide a plot for the RKC density of states.

\section{Regularization and Model Parameters}

Due to scattering of the quarks off the crystal the quasi-particle energies in the inhomogeneous phase cannot be labelled by a conserved three-momentum. Thus 3-dimensional momentum cutoff regularization is not suitable here and leads to regularization artifacts as seen in Refs. [21, 25]. There the authors isolated the wave-number independent part of the grand potential and applied a momentum cutoff only to this part, which then leads to an misalignment of the Lifshitz point compared to the tricritical point, in contradiction to the general Ginzburg-Landau result of Ref. [22], where both the Lifshitz point and the tricritical point are found to coincide. This regularization artifact can be prevented by following Ref. [26] and applying a PauliVillars regularization scheme, which acts on the energy spectrum instead. In 
our case this amounts to a replacement of

$$
\epsilon_{ \pm}=\sqrt{(E \pm \mu)^{2}+|\Delta|^{2}} \rightarrow \sum_{j} c_{j} \sqrt{\left(\sqrt{E^{2}+j \Lambda^{2}} \pm \mu\right)^{2}+|\Delta|^{2}}
$$

in the temperature-independent part of the thermodynamic potential. Following Ref. [77], we take three regulators with the coeffcients $c_{0}=-c_{3}=$ $1, c_{1}=-c_{2}=-3$, and fix the cutoff parameter $\Lambda$ together with the coupling constant $G$ by fitting [82] the constituent quark mass in vacuum to $M=300 \mathrm{MeV}$ and the vacuum pion decay constant to its (approximate) value in the chiral limit, $f_{\pi}=88 \mathrm{MeV}$, which yields $\Lambda=757.048 \mathrm{MeV}$ and $G \Lambda^{2}=6.002$.

The diquark coupling $G_{\Delta}$ can be obtained from a Fierz transformation of the interaction part of the Lagrangian. In a color-current interaction model based on one-gluon exchange, it is found to be $G_{\Delta}=3 / 4 G$ [56]. However, here we will treat $G_{\Delta}$ as a free parameter since its value can not be reliably associated with a well-measured observable. We therefore present phase diagrams for four different diquark couplings: $G_{\Delta}=0,0.3 G, G / 2$, and $3 G / 4$.

\subsection{Results}

Now let us present the phase diagrams for the CDW and RKC Ansatz by minimizing the grand potentials given by Eqs. (3.27) and (3.30), respectively. For the CDW Ansatz we need to minimize the grand potential with respect to $M, q$, and the diquark gap $\Delta$, while for the RKC Ansatz we have the parameters $D, \Delta$, and $v$. As a side remark, the minimization procedure for the RKC Ansatz is numerically more difficult because extremely small changes in the parameter $v$ lead to large changes in the shape of the inhomogeneous condensate and thus also in the grand potential. Therefore, we need to perform both the numerical integration and minimization with significantly higher precision compared to the CDW Ansatz.

The resulting phase diagrams are shown in Fig. 3.5, where in total five different phases can be found:

- The chiral symmetry-restored phase (R): $M=0, q=0, \Delta=0$.

- The homogeneous chiral symmetry-broken phase (Ch): $M \neq 0, q=$ $0, \Delta=0$.

- The inhomogeneous chiral phase (InhCh): $M \neq 0, q \neq 0, \Delta=0$.

- The 2SC phase (2SC): $M=0, q=0, \Delta \neq 0$.

- The coexistence phase (C): $M \neq 0, q \neq 0, \Delta \neq 0$.

Regions with inhomogeneous chiral condensates are bounded by green lines and regions with a nonvanishing 2SC condensate by blue lines. Solid lines represent the phase boundaries for the CDW Ansatz, while dashed lines 
correspond to the RKC Ansatz. The orange lines separate the homogeneous chiral symmetry-broken phase from the restored phase. In all cases where an inhomogeneous phase occurs, the tricritical point coincides with the Lifshitz point, in agreement with the results of Ref. [22].

As a first observation in Fig. 3.5 we would like to highlight that the specific Ansatz for the inhomogeneous condensate affects the phase boundaries only slightly. For example, in the upper left panel the inhomogeneous region for the RKC Ansatz extends to slightly smaller $\mu$ compared to the CDW Ansatz, which is in agreement with previous NJL studies without colorsuperconductivity, see, e.g., Ref. [83].

Coexistence phases are found for $G_{\Delta}=0.3 G$ and $G_{\Delta}=0.5 G$, as seen in the upper right and the lower left panels in Fig. 3.5. This is a nontrivial result because it could just as easily have been the case that either the pure inhomogeneous phase was energetically preferred, which would prevent diquark condensation within the inhomogeneous phase, or that the pure 2SC phase was energetically preferred over a pure inhomogeneous chiral phase, which would then have removed the low-temperature part of the inhomogeneous phase. Indeed, it is seen that as we increase the diquark coupling, the 2SC phase increases in size and replaces the inhomogeneous chiral phase. This of course is no surprise because increasing the diquark coupling means that Cooper pairs are more bounded in a more attractive potential and thus stabilize the system. The choice of the specific inhomogeneous condensate also affects the boundaries of coexistence phase slightly. The RKC Ansatz leads to a smaller coexistence phase, which can be explained by the fact that the RKC crystal is energetically preferred over the CDW and therefore the RKC inhomogeneous phase can extend to smaller temperatures before competition with the 2SC phase sets in.

In this context, another interesting observation is that the coexistence phase sets in at smaller values of the chemical potential than a pure 2SC phase without inhomogeneous chiral condensate. This effect is rather small, and it is analogous to the fact that the phase transition between the homogeneous chiral symmetry-broken phase to the inhomogeneous phase occurs at slightly smaller $\mu$ than the first-order phase transition between the homogeneous chiral symmetry-broken and the restored phase [84].

This can be seen in Fig. 3.6, where the grand potentials for the different homogeneous and inhomogeneous phases are compared. Each line represents a local extremum associated with the phase denoted in the legend of the figure. Intersections of two lines correspond to first-order phase transitions between the two respective phases, while converging lines characterize two converging extrema, corresponding to a second-order phase transition.

With Fig. 3.6 we can verify the result of Ref. [83]. There the authors found that, while for the CDW Ansatz we have a first-order phase transition between the chiral symmetry-broken phase to the inhomogeneous chiral phase, this becomes a second-order transition when employing the RKC Ansatz. This qualitative finding remains valid when including a 2SC phase, but here the second-order transition is to the coexistence phase instead of to the inhomogeneous chiral phase. 
The different nature of the chiral phase transitions for the CDW and the RKC Ansatz can be also seen in the three-dimensional plots in Figs. 3.7 and 3.8. While both plots for the CDW and the RKC Ansatz look very similar, one can see the different behavior for the wave number close to the $\mathrm{Ch}-\mathrm{C}$ phase boundary. For the CDW Ansatz, the wave number jumps from zero to a finite value, corresponding to the first-order transition, while for the RKC Ansatz, it increases very steeply but continuously at the second-order transition. One can also see a small discontinuous onset of the diquark gap for the CDW Ansatz, while the onset becomes continuous for the RKC case.
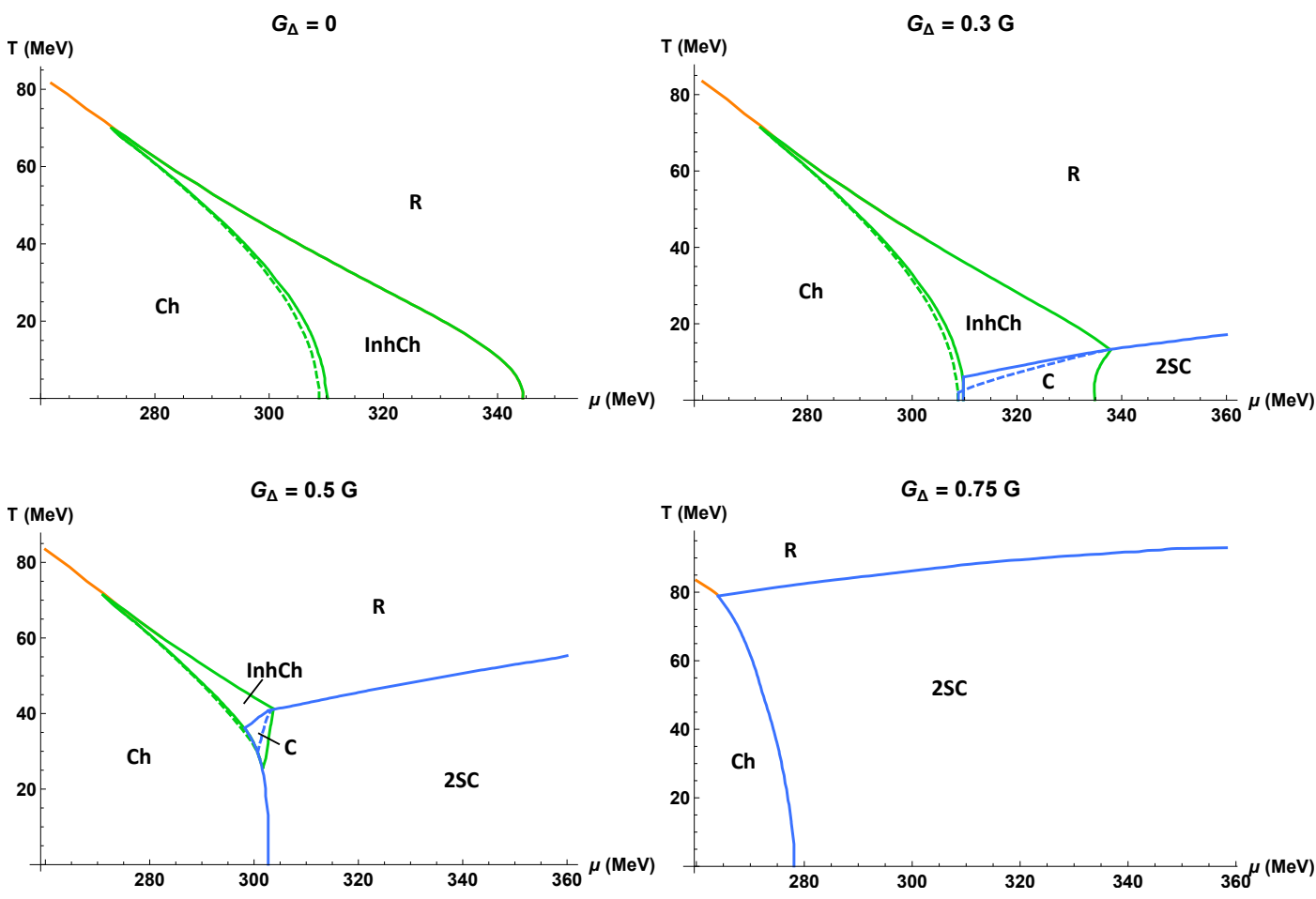

FIGURE 3.5: The phase boundaries for different diquark couplings $G_{\Delta}=$ $0,0.3 G, G / 2,3 G / 4$. The solid lines correspond to the phase transition lines for the CDW Ansatz, while the dashed lines are associated with the RKC Ansatz. Where only solid lines are visible, the phase boundaries for both Ansätze coincide. 


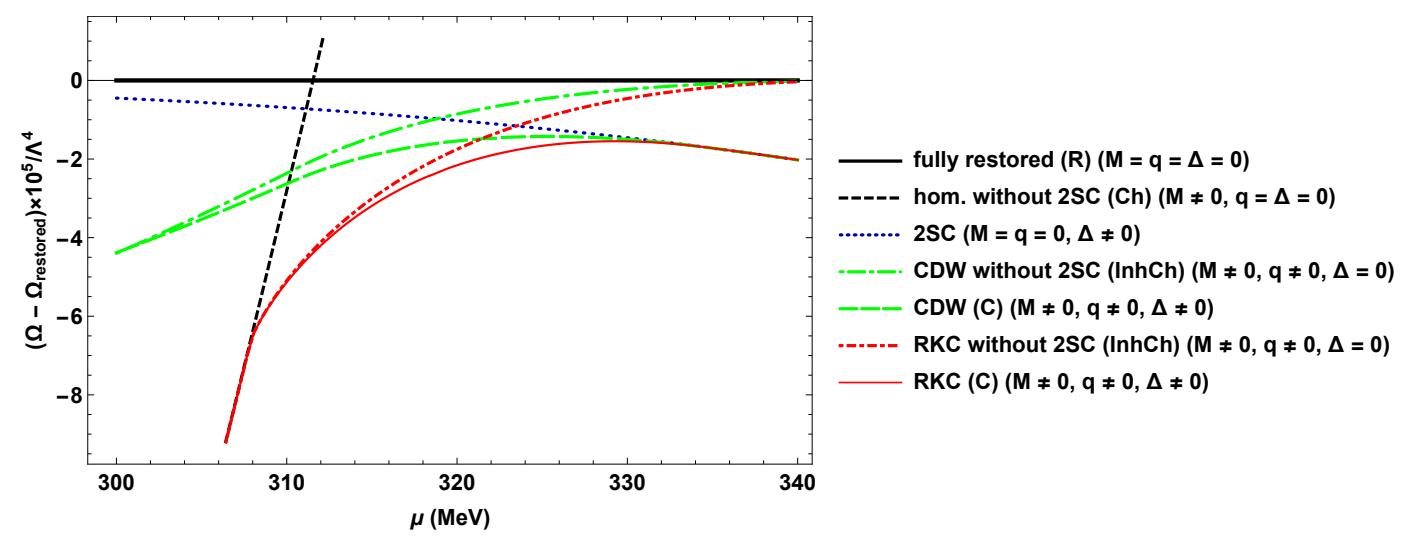

FIGURE 3.6: Comparison of the grand potentials for different scenarios at $T=0 \mathrm{MeV}$ and for $G_{\Delta}=0.3 \mathrm{G}$. Two intersecting lines represent first-order transitions between the two respective phases, while converging lines characterize second-order phase transitions. For each case we subtract the potential of the fully restored ( $M=q=$ $\Delta=0)$ solution.

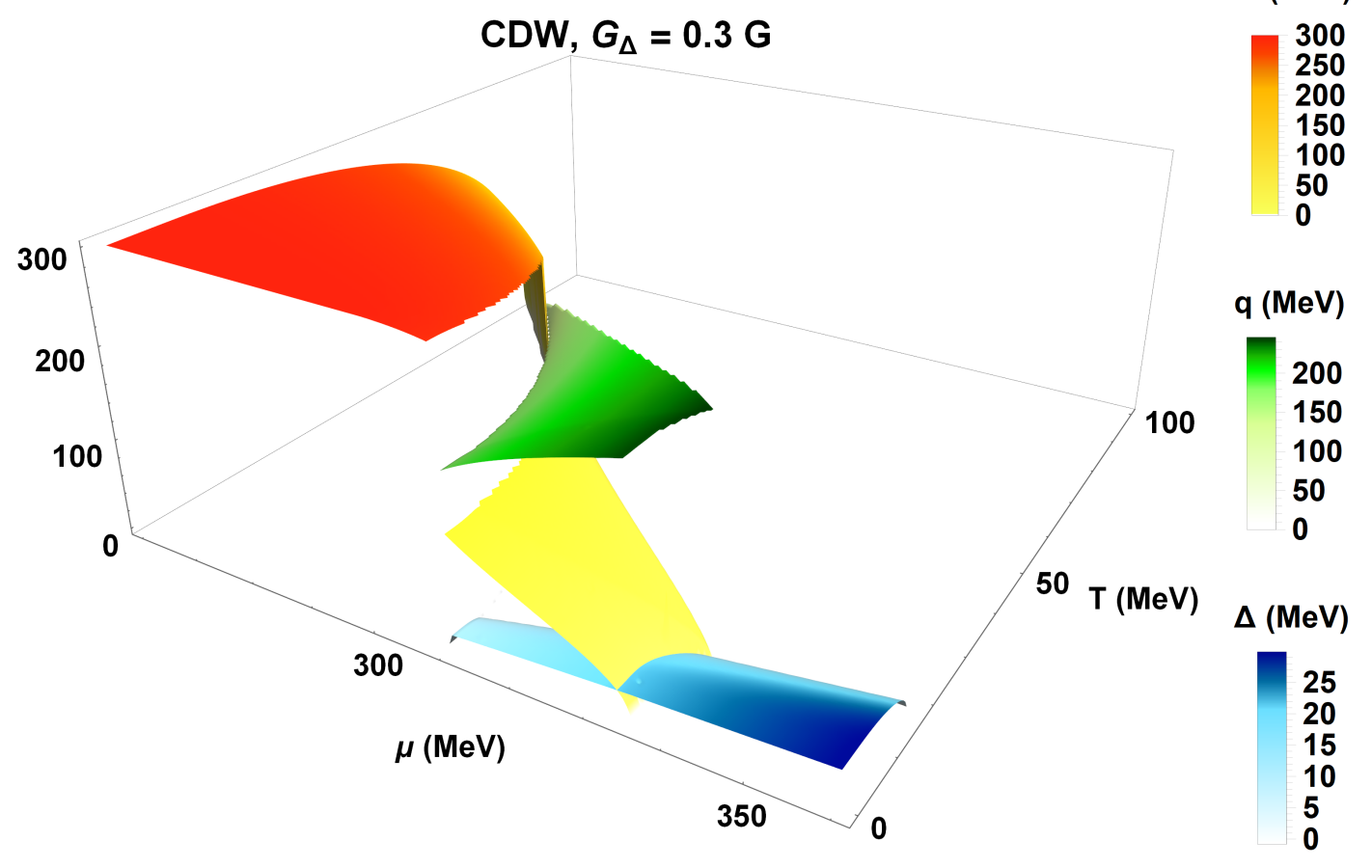

FIGURE 3.7: A detailed view of the order parameters for the CDW for $G_{\Delta}=0.3 G$. 


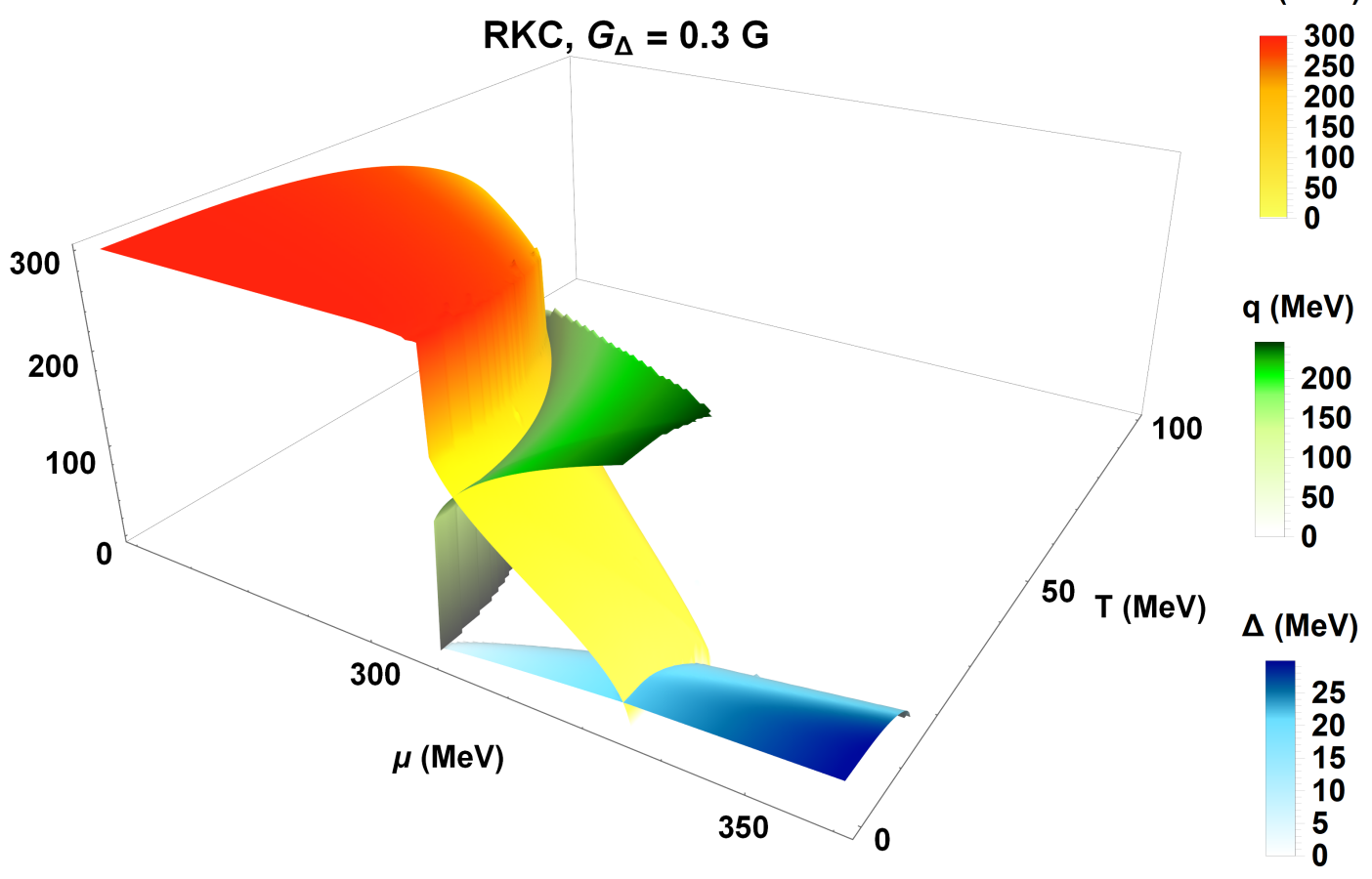

FIGURE 3.8: A detailed view of the order parameters for the RKC for $G_{\Delta}=0.3 G$.

Finally, in the lower right panel of Fig. 3.5 we increased the diquark coupling to $G_{\Delta}=3 G / 4$. For this parameter set, neither an inhomogeneous chiral phase nor a coexistence phase is found and therefore the phase boundaries for both the CDW and the RKC Ansatz exactly align. Note that this is in contrast to Ref. [21], where the inhomogeneous chiral phase and the coexistence phase were also found in the case $G_{\Delta}=3 G / 4$. The reason for the discrepancy most likely is because of the different regularization scheme that was used in Ref. [21].

As mentioned in the beginning of this discussion, the major takeaway is the observation that the phase boundaries are only slightly affected by the choice of the specific inhomogeneous Ansatz for the chiral order parameter. This knowledge combined with the finding of Ref. [83], according to which the RKC Ansatz is the energetically most preferred inhomogeneous Ansatz that has been found so far, while the CDW is the least favored, this might be an important insight for future studies in this direction, where it might not be technically possible to choose the energetically most preferred Ansatz. Especially for exploratory studies it might be sufficient to employ the CDW, which, because of its extremely nice properties, is often the first choice when studying inhomogeneous phases. 



\section{Chapter 4}

\section{The Functional Renormalization Group}

In this chapter we will introduce the functional renormalization group and substantiate why this framework is so important for the purpose of this thesis. Subsequently, we will use the functional renormalization group to study the quark-meson-diquark model in its simplest truncation, the so-called local potential approximation (LPA). Most of the detailed calculations are given in Appendices D and E.

\subsection{The Concept}

The idea of the functional renormalization group goes back to Kadanoff's block-spin model [85, 86]. This model is a two-dimensional Ising model, where the microscopic coupling between two spins is the inverse temperature, i.e., $K=1 / T$. The question is now the following: How does the coupling of the effective degrees of freedom change when the scale of the system changes? To that aim, one combines several spins to a new block, e.g., each $3 \times 3$ spin square configuration becomes a new block, and then one takes the average of each block ${ }^{1}$. This so-called coarse graining procedure reduces the degrees of freedom of the system (in this example by a factor of nine) and thus also its scale. Using the block-spin transformation we can determine the new effective Hamiltonian for the spin blocks, which has the same shape but a different coupling constant. If we now apply the block-spin transformation repeatedly until the desired scale is reached, we have calculated the effective coupling for that scale starting from the microscopic theory.

Often, when the block-spin transformation is performed many times, the effective coupling flows toward a fixed point, where it does not change anymore even with further iterations of the block-spin transformation. In studies of fundamental theories these fixed points are very important, because they are a necessary condition to guarantee that the couplings of the theory do not diverge in the UV or IR. One of the most prominent examples is the Gaussian

\footnotetext{
${ }^{1}$ Incidentally, this is analogous to pooling as commonly used in convolutional neural networks [87]. The analogy for the example of this specific block-spin transformation becomes exact for zero padding and stride equal to three.
} 
fixed point ${ }^{2}$ of QCD, where the beta function vanishes at increasingly large energies, which is the reason for the asymptotic freedom of QCD.

The functional renormalization-group formalism is inspired by Kadanoff's block-spin transformation but is formally significantly different. The FRG has its roots in the Wilson renormalization group [88] which can be seen as the continuum version of the block-spin transformation. Instead of averaging spins on a lattice, the Wilson renormalization group successively integrates out heavy fields in the path integral up to a certain energy scale. Technically, this works as follows: Consider the generating functional in Euclidean spacetime

$$
\mathcal{Z}_{\Lambda}=\mathcal{N} \int_{|p|=0}^{\Lambda} \mathcal{D} \phi \exp \left\{-S_{\Lambda}[\phi]+\int_{x} \phi(x) J(x)\right\},
$$

where $S_{\Lambda}$ is a classical action defined at the energy scale $\Lambda$. Next, we introduce another cutoff $\Lambda^{\prime}<\Lambda$ and define soft and hard modes denoted as $\tilde{\phi}$ and $\hat{\phi}$, respectively:

$$
\begin{aligned}
& \tilde{\phi}(p)=\phi(p) \theta\left(\Lambda^{\prime}-|p|\right) \\
& \hat{\phi}(p)=\phi(p)\left[1-\theta\left(\Lambda^{\prime}-|p|\right)\right] \\
& \phi(p) \equiv \tilde{\phi}(p)+\hat{\phi}(p) .
\end{aligned}
$$

Next, if we write the generating functional in terms of the soft and hard modes, and subsequently integrate out the hard modes, we obtain

$$
\begin{aligned}
\mathcal{Z}_{\Lambda}[J] & =\mathcal{N} \int_{|p|=0}^{\Lambda^{\prime}} \mathcal{D} \tilde{\phi} \int_{|p|=\Lambda^{\prime}}^{\Lambda} \mathcal{D} \hat{\phi} \exp \left\{-S_{\Lambda}[\tilde{\phi}, \hat{\phi}]+\int_{x}[\tilde{\phi}+\hat{\phi}] J(x)\right\} \\
& =\mathcal{N} \int_{|p|=0}^{\Lambda^{\prime}} \mathcal{D} \tilde{\phi} \exp \left\{-S_{\Lambda^{\prime}}[\tilde{\phi}]+\int_{x} \tilde{\phi} J(x)\right\},
\end{aligned}
$$

where the new action $S_{\Lambda^{\prime}}$ has been defined via

$$
\mathrm{e}^{-S_{\Lambda^{\prime}}[\tilde{\phi}, J]}=\int_{|p|=\Lambda^{\prime}}^{\Lambda} \mathcal{D} \hat{\phi} \exp \left\{-S_{\Lambda}[\tilde{\phi}, \hat{\phi}]+\int_{x} \hat{\phi} J(x)\right\}
$$

This is a Schwinger functional and contains quantum corrections, which, on the one hand, modify the already existing couplings of the classical theory and, on the other hand, induce all new couplings that respect the symmetry of the classical theory. If we were in a regime where the couplings are small, it would be possible to neglect the new couplings using perturbation theory, but for strongly coupled systems this is an invalid approximation. Therefore, the idea is now to first keep all the new couplings and find an equation that describes how the couplings change when the generating functional is

\footnotetext{
${ }^{2}$ The Gaussian fixed point is a fixed point at which the coupling vanishes.
} 


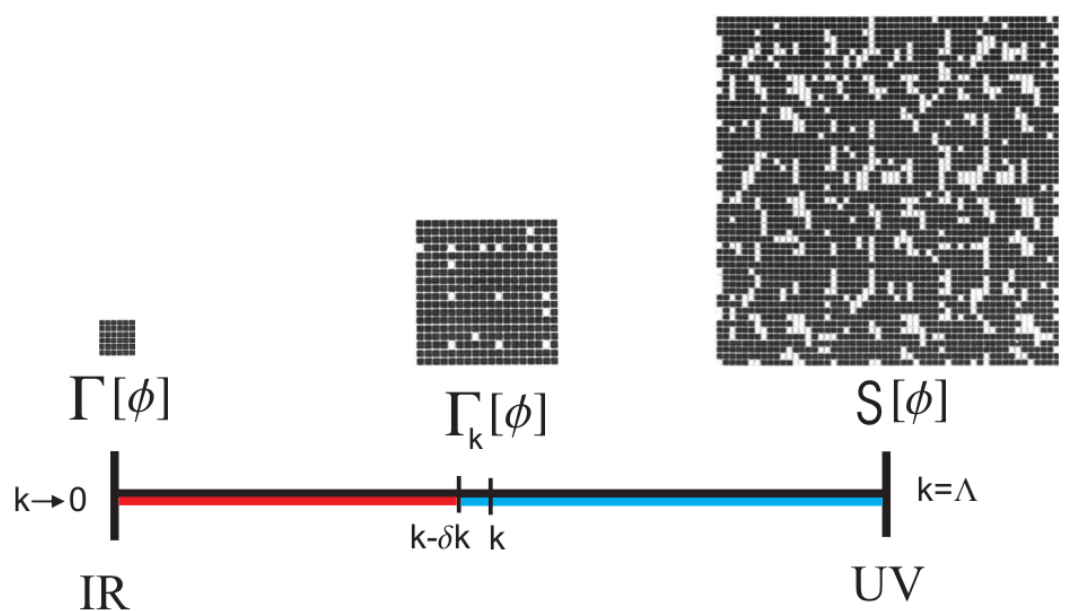

FIGURE 4.1: The scale-dependent effective average action interpolates between the classical action in the UV and the effective action in the IR. This analogous to the Kadanoff block-spin transformation. In the UV we have many degrees of freedoms, which are integrated out until we have only a few effective degrees of freedom left in the IR.

integrated out momentum shell by momentum shell. Subsequently, when we have found such an equation, we can still apply an approximation that is most suitable for the investigated phenomenology in question. Thus, the idea is now to further systematize the idea of the Wilson renormalization group by introducing a scale-dependent effective action $\Gamma_{k}$, where $k$ is a continuous parameter which denotes the momentum scale at which all higher momenta $p^{2}>k^{2}$ are integrated out. In other words, we want the scale-dependent action to be a functional that interpolates between the classical action $S[\phi]$ at high energies and the full quantum effective action $\Gamma[\phi]$ at low energies. This interpolation can be described by a partial differential equation called the exact renormalization group equation, also known as the Wetterich equation.

\subsection{The Exact Renormalization Group Equation}

Now that we have explained the conceptual ideas behind the FRG, we can mathematically derive the Wetterich equation (See also Refs. [67, 89, 90, 91, 92, 93] for derivations in literature). The idea is the following: We render the action scale dependent by adding a scale-dependent mass term:

$$
S[\phi] \longrightarrow S[\phi]+\Delta S_{k}[\phi],
$$

with the newly introduced term defined as:

$$
\Delta S_{k}[\phi]=\frac{1}{2} \int_{p} \phi_{a}^{\mathrm{T}}(-p) R_{k}^{a b}(p) \phi_{b}(p)
$$


where summation over same indices is implied. $\phi_{a}$ is a field-space vector of bosonic and fermionic fields and $R_{k}^{a b}$ is the so-called regulator in field space, which is defined such that both IR- and UV-regularization is guaranteed while at the same time respecting the boundary conditions of the effective average action, which are defined as

$$
\Gamma_{k \rightarrow \Lambda}=S, \quad \Gamma_{k \rightarrow 0}=\Gamma .
$$

The effective average action interpolating between the classical action $S$ and the quantum effective action $\Gamma$ is derived from the scale-dependent generating functional,

$$
Z_{k}[J]=\int \mathcal{D}[\phi] \exp \left(-S[\phi]-\Delta S_{k}[\phi]+\int J_{a} \phi_{a}\right)
$$

via Legendre transformation of the scale-dependent Schwinger functional

$$
\begin{aligned}
W_{k}[J] & =\ln \left(Z_{k}[J]\right), \\
\rightarrow \Gamma_{k}[\Phi] & =\sup _{J}\left(-W_{k}[J]+\int_{a} J_{a} \Phi_{a}\right)-\Delta S_{k}[\Phi] .
\end{aligned}
$$

Note that $\Phi$ has been introduced as a consequence of the Legendre transformation and is defined by

$$
\Phi_{a}(x)=\left\langle\phi_{a}(x)\right\rangle_{J=J_{\text {sup }}}=\left.\frac{\delta}{\delta J_{a}(x)} W_{k}[J]\right|_{J=J_{\text {sup }}} .
$$

In the following we will always use $J \equiv J_{\text {sup. From Eq. (4.12) the constraints }}$ of the regulator are derived:

1. $\lim _{p^{2} / k^{2} \rightarrow 0} R_{k}\left(p^{2}\right)>0$,

2. $\lim _{k^{2} / p^{2} \rightarrow 0} R_{k}\left(p^{2}\right)=0$ ，

3. $\lim _{k^{2} \rightarrow \Lambda \rightarrow \infty} R_{k}\left(p^{2}\right)=\infty$.

To recapitulate, since the regulator can be seen as a scale-dependent effective mass, the first property ensures that modes with $p^{2} \lesssim k^{2}$ acquire a mass resulting in the decoupling of the soft modes, i.e., these soft fluctuations are suppressed. The second property needs to be fulfilled in order to obtain the correct physical limit in which the theory should not depend on an arbitrary regulator. This ensures the recovery of the full quantum effective action, $\lim _{k \rightarrow 0} \Gamma_{k}[\phi]=\Gamma[\phi]$. Finally, the last property is needed to obtain the correct UV limit, i.e., no fluctuations contribute and the theory is described by the classical action, $\lim _{k \rightarrow \Lambda \rightarrow \infty} \Gamma_{k}[\phi]=S[\phi]$. 
In the next step let us modify the effective average action by adding the scale-dependent mass term:

$$
\tilde{\Gamma}_{k}[\Phi]=\Gamma_{k}[\Phi]+\Delta S_{k}[\Phi]=-W_{k}[J]+\int_{a} J_{a} \Phi_{a}
$$

Now we would like to investigate how this quantity changes as the flow scale is varied:

$$
\begin{aligned}
\partial_{k} \tilde{\Gamma}_{k}[\Phi] & =-\partial_{k} W_{k}[J] \\
& =-\frac{\partial_{k} Z_{k}[J]}{Z_{k}[J]} \\
& =-\frac{1}{Z_{k}[J]} \int \mathcal{D}[\phi]\left(-\partial_{k} \Delta S_{k}[\phi]\right) \exp \left(-S[\phi]-\Delta S_{k}[\phi]+\int J_{a} \phi_{a}\right) .
\end{aligned}
$$

Note that this result is simply the expectation value of $\partial_{k} \Delta S_{k}[\phi]$. This can be further simplified to

$$
\begin{aligned}
\partial_{k} \tilde{\Gamma}_{k}[\Phi] & =\left\langle\partial_{k} \Delta S_{k}[\phi]\right\rangle \\
& =\frac{1}{2} \int_{p} \partial_{k} R_{k}^{a b}(p)\left\langle\phi_{a}(-p) \phi_{b}(p)\right\rangle \\
& =\frac{1}{2} \int_{p} \partial_{k} R_{k}^{a b}(p)\left(\left\langle\phi_{a}(-p) \phi_{b}(p)\right\rangle_{\text {connected }}+\left\langle\phi_{a}(-p)\right\rangle\left\langle\phi_{b}(p)\right\rangle\right) \\
& =\frac{1}{2} \int_{p} \partial_{k} R_{k}^{a b}(p)\left(W_{k, a b}^{(2)}(p)+\left\langle\phi_{a}(-p)\right\rangle\left\langle\phi_{b}(p)\right\rangle\right) \\
& =\frac{1}{2} \int_{p} \partial_{k} R_{k}^{a b}(p) W_{k, a b}^{(2)}(p)+\partial_{k} \Delta S_{k}[\Phi] .
\end{aligned}
$$

Thus, inserting this result back into Eq. (4.14) yields

$$
\partial_{k} \Gamma_{k}[\Phi]=\partial_{k} \tilde{\Gamma}_{k}[\Phi]-\partial_{k} \Delta S_{k}[\Phi]=\frac{1}{2} \int_{p} \partial_{k} R_{k}^{a b}(p) W_{k, a b}^{(2)}(p)
$$

From Eq. (4.13) it follows that

$$
\frac{\delta}{\delta J_{b}(y)} \Phi_{a}(x)=W_{k, a b}^{(2)}(x, y),
$$

and from Eq. (4.12) we obtain

$$
\frac{\delta}{\delta \Phi_{b}(y)} J_{a}(x)=\left(\Gamma_{k}^{(2)}+R_{k}\right)_{a b}(x, y) .
$$

Now, using the identity 


$$
\begin{aligned}
\delta_{a b} \delta(x-y) & =\frac{\delta J_{a}(x)}{\delta J_{b}(y)}=\int_{x^{\prime}} \frac{\delta J_{a}(x)}{\delta \Phi_{a^{\prime}}\left(x^{\prime}\right)} \frac{\delta \Phi_{a^{\prime}}\left(x^{\prime}\right)}{\delta J_{b}(y)} \\
& =\int_{x^{\prime}}\left(\Gamma_{k}^{(2)}+R_{k}\right)_{a a^{\prime}}\left(x, x^{\prime}\right) W_{k, a^{\prime} b}^{(2)}\left(x^{\prime}, y\right)
\end{aligned}
$$

we have shown that

$$
W_{k}^{(2)}=\left(\Gamma_{k}^{(2)}+R_{k}\right)^{-1} \text {. }
$$

Inserting this identity into Eq. (4.16) we obtain the Wetterich equation:

$$
\begin{aligned}
\partial_{k} \Gamma_{k}[\Phi] & =\frac{1}{2} \int_{p} \partial_{k} R_{k}^{a b}(p) W_{k, a b}^{(2)}(p) \\
& =\frac{1}{2} \int_{p} \frac{\partial_{k} R_{k}^{a b}(p)}{\left(\Gamma_{k}^{(2)}+R_{k}\right)_{a b}(p)},
\end{aligned}
$$

or in an equivalent notation

$$
\partial_{k} \Gamma_{k}[\phi]=\frac{1}{2} S \operatorname{Tr}\left[\frac{\partial_{k} R_{k}}{\Gamma_{k}^{(2)}[\phi]+R_{k}}\right]=\sum_{\eta= \pm} \frac{\eta}{2} \int_{p} \frac{\partial_{k} R_{k}\left(p^{2}\right)}{\Gamma_{k}^{(2)}(p,-p)+R_{k}\left(p^{2}\right)}
$$

where STr is a trace with an additional minus sign for fermions. Equivalently, we have $\eta=1$ for bosons and $\eta=-1$ for fermions.

Throughout this thesis we will use the so-called Litim regulator [94], which is widely used in many FRG studies because it has properties that are very useful in analytic computations. The Litim regulator is defined as

$$
R_{k}\left(p^{2}\right)= \begin{cases}\left(k^{2}-p^{2}\right) \theta\left(k^{2}-p^{2}\right), & \text { in the bosonic subspace } \\ i p\left(\sqrt{\frac{k^{2}}{p^{2}}}-1\right) \theta\left(k^{2}-p^{2}\right), & \text { in the fermionic subspace }\end{cases}
$$

The Wetterich equation is exact in the sense that no approximations have been made during the derivation. However, since the right-hand side of the Wetterich equation depends on the second functional derivative of the effective action, we need to access the information of the full flow dependence of $\Gamma_{k}^{(2)}$. This information can in principle be obtained by taking two functional derivatives of the Wetterich equation with respect to $\phi$ : 


$$
\begin{aligned}
\partial_{k} \Gamma_{k}^{(2)}= & \frac{1}{2} S \operatorname{Tr}\left[\partial_{k} R_{k} \frac{1}{\Gamma_{k}^{(2)}+R_{k}} \Gamma_{k}^{(3)} \frac{1}{\Gamma_{k}^{(2)}+R_{k}} \Gamma_{k}^{(3)} \frac{1}{\Gamma_{k}^{(2)}+R_{k}}\right] \\
& -\frac{1}{2} S \operatorname{Tr}\left[\frac{1}{\Gamma_{k}^{(2)}+R_{k}} \Gamma_{k}^{(4)} \frac{1}{\Gamma_{k}^{(2)}+R_{k}}\right]
\end{aligned}
$$

where we suppressed the momentum dependence. The flow of the scaledependent two-point function thus depends on the three- and four-point function. Therefore, continuing taking functional derivatives of the Wetterich equation yields an infinite tower of coupled differential equations. Hence, without some truncation scheme it is not possible to solve the Wetterich equation analytically.

\subsection{Truncations}

In general, we can write the scale-dependent effective average action as

$$
\Gamma_{k}[\phi]=\sum_{n} g_{n, k} \mathcal{O}_{n}\left[\phi, \partial_{\mu} \phi\right]
$$

where $g_{n, k}$ are the running coupling constants and $\mathcal{O}_{n}\left[\phi, \partial_{\mu} \phi\right]$ are the operators consisting of fields and derivatives of fields. Note, this is an infinite sum as quantum fluctuations will produce all possible operators that respect the symmetries of the underlying theory. As mentioned before, we cannot solve the Wetterich equation with infinitely many operators, but if we consider Eq. (4.25) three possible truncation schemes present ourselves:

1. Loop expansion: Expanding Eq. (4.25) for small $g_{n, k}$, which is related to the well-known perturbation theory

2. Vertex expansion: Expanding in powers of fields

3. Derivative expansion: Expanding the effective average action with respect to the powers of derivatives

Throughout this thesis we will be using the derivative expansion to truncate the effective action. Taking the $O(N)$ model as example, the Ansatz for the effective average action in the derivative expansion is written as

$$
\Gamma_{k}=\int_{x}\left[U_{k}\left(\phi^{2}\right)+\frac{1}{2} Z_{k}(\phi) \partial_{\mu} \phi \partial^{\mu} \phi+\mathcal{O}\left(\partial^{4}\right)\right]
$$

where $Z_{k}(\phi)$ is the scale- and field-dependent wave-function renormalization constant and $U_{k}\left(\phi^{2}\right)$ is the effective potential containing all $O(N)$-symmetric 
interaction terms. Using the Wetterich equation, again a tower of coupled flow equations needs to be solved:

$$
\begin{aligned}
& \partial_{k} U_{k}\left(\phi_{0}\right)=\left.\frac{1}{2 \operatorname{Vol}_{d}} \operatorname{Tr} \frac{\partial_{k} R_{k}}{\Gamma_{k}^{(2)}+R_{k}}\right|_{\phi=\phi_{0}}, \\
& \partial_{k} Z_{k}\left(\phi_{0}\right)=\frac{1}{2 \operatorname{Vol}_{d}} \partial_{p^{2}}\left(\left.\frac{\delta}{\delta \phi(-p)} \frac{\delta}{\delta \phi(q)} \operatorname{Tr} \frac{\partial_{k} R_{k}}{\Gamma_{k}^{(2)}+R_{k}}\right|_{\phi=\phi_{0}, p=q}\right)_{p=0},
\end{aligned}
$$

where the dots denote the flow equations for the wave-function renormalizations of the higher-order kinetic terms. In this thesis we will truncate the effective average action such that only the first equation, Eq. (4.27), needs to be solved. This corresponds to the case where only the local kinetic term contributes and the wave-function renormalization is set to $Z_{k}=1$. This approximation is called the local potential approximation (LPA).

Of course by omitting all the other terms, we assume that the contributions of these terms are negligible. However, there is no rigorous a priori reason that this needs to be the case and it is an ongoing challenge for the FRG community to understand at which order the derivative expansion starts to be reliable. A notable study in this direction is for example Ref. [70], where the authors showed in the quark-meson-diquark model, that the LPA yields significantly different results compared to improved higher-order truncations. However, in that study the UV parameters have been chosen such that the highest-order truncations yield the correct IR results and then they used the same starting values for the LPA. But it is still possible to choose suitable UV parameters such that the LPA also yields the correct IR observables. The model then has a more phenomenological nature as the UV parameters are tuned to agree with observation while for higher-order truncations the UV parameters could be seen as a prediction from QCD.

Regardless of whether or not the LPA is suitable as a phenomenological approach to yield an accurate description of low-energy QCD, it is at least an improvement over the widely used MFA (for example in studies of the QCD phase diagram). For instance, employing the FRG in the LPA will allow for a qualitative study of the effect of bosonic fluctuations, whereas the MFA only incorporates fermionic fluctuations. For example, studies on inhomogeneous phases have been mainly done in the MFA [95], as we also did in the last chapter. Comparing the results of these studies with an equivalent FRG study could lead to a better understanding of the effects of bosonic fluctuations on inhomogeneous phases. Furthermore, presenting an LPA study can serve as an important starting point to motivate an FRG study with improved truncation. Comparing results of the LPA study with one with improved truncation will lead to important insight on the importance of higher-order corrections. 


\subsection{Flow of the Effective Potential}

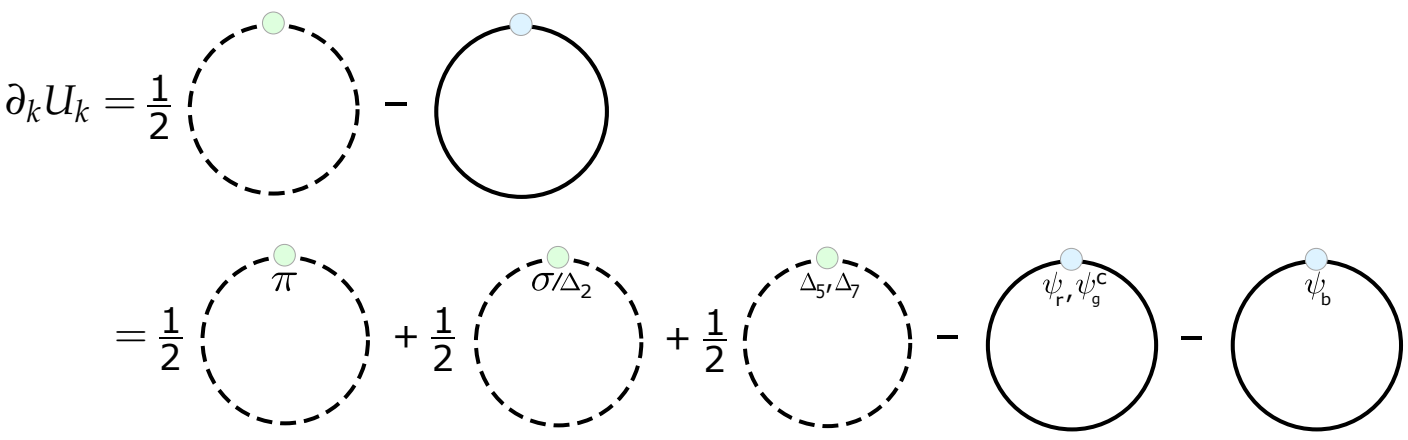

FIGURE 4.2: Diagrammatic representation of the different contributions to the flow of the effective potential. In the first line we have two loops representing the bosonic (where the green blob denotes the bosonic regulator insertion $\partial_{k} R_{k, \mathrm{~B}}$ ) and the fermionic (where the blue blob denotes the fermionic regulator insertion $\partial_{k} R_{k, \mathrm{~F}}$ ) contributions, respectively. In the second line we further unfolded the different contributions in field space.

In this section we present the flow equation for the effective potential of the quark-meson-diquark model. The scale-dependent effective action of this model in the LPA is defined as

$$
\begin{aligned}
& \Gamma_{k}^{\mathrm{QMD}}[\Phi]=\int d^{4} x\left\{\bar{\psi}\left[i \not \partial-\mu \gamma_{0}+h_{\phi}\left(\sigma+i \gamma_{5} \vec{\tau} \cdot \vec{\pi}\right)\right] \psi\right. \\
& +\frac{h_{\Delta}}{2}\left[\Delta^{* a} \psi^{\mathrm{T}} C \tau_{2} \epsilon^{a} \gamma_{5} \psi-\Delta^{a} \bar{\psi} \gamma_{5} \epsilon^{a} \tau_{2} C \bar{\psi}^{\mathrm{T}}\right]+\frac{1}{2}\left[\left(\partial_{\mu} \sigma\right)^{2}+\left(\partial_{\mu} \vec{\pi}\right)^{2}\right] \\
& \left.+\frac{1}{2}\left[\left(\partial_{v}-2 \delta_{\nu 0} \mu\right) \Delta^{* a}\right]\left[\left(\partial_{v}+2 \delta_{v 0} \mu\right) \Delta^{a}\right]+U_{k}\left(\phi^{2},\left|\Delta_{a}\right|^{2}\right)-c \sigma\right\}
\end{aligned}
$$

Here we simply rendered the effective potential scale dependent and defined $\phi \equiv(\sigma, \vec{\pi})$. In an improved truncation one could render the couplings $h_{\phi}$ and $h_{\Delta}$ scale-dependent, include a running wave-function renormalization $Z_{k}$ or include interactions of order $\mathcal{O}\left(\partial^{4}\right)$. These possibilities are all neglected here. In the LPA, one can then obtain the effective potential by taking Eq. (4.30) at a constant field configuration

$$
\Phi=\left(\begin{array}{c}
\sigma \\
\vec{\pi} \\
\operatorname{Re} \Delta_{a} \\
\operatorname{Im} \Delta_{a} \\
\bar{\psi} \\
\psi
\end{array}\right) \rightarrow \Phi_{0}=\left(\begin{array}{c}
\sigma_{0} \\
0 \\
\delta_{a 2} \Delta_{0} \\
0 \\
0 \\
0
\end{array}\right)
$$

In the following we will rename the vacuum expectation values $\sigma_{0} \rightarrow \sigma$ and $\Delta_{0} \rightarrow \Delta . \sigma$ is the order parameter for spontaneous chiral symmetry breaking 
and $\Delta$ is the diquark condensate and acts as order parameter for the twoflavor color-superconducting phase. The flow equation is derived in App. D:

$$
\begin{aligned}
\partial_{k} U_{k}= & \frac{k^{4}}{12 \pi^{2}}\left[\frac{3}{E_{\pi, k}} \operatorname{coth} \frac{E_{\pi, k}}{2 T}+\sum_{i=1}^{3} \frac{\alpha_{0}-\alpha_{1} z_{i}^{2}+3 z_{i}^{4}}{\left(z_{i+1}^{2}-z_{i}^{2}\right)\left(z_{i+1}^{2}-z_{i}^{2}\right) z_{i}} \operatorname{coth} \frac{z_{i}}{2 T}\right. \\
& \left.+\frac{2}{\tilde{E}_{\Delta, 0, k}}\left(\operatorname{coth} \frac{\tilde{E}_{\Delta, 0, k}-2 \mu}{2 T}+\operatorname{coth} \frac{\tilde{E}_{\Delta, 0, k}+2 \mu}{2 T}\right)\right] \\
& -\frac{k^{4}}{3 \pi^{2}}\left[2 \sum_{ \pm} \frac{1 \pm \frac{\mu}{E_{k}}}{E_{k}^{ \pm}} \tanh \frac{E_{k}^{ \pm}}{2 T}+\frac{1}{E_{k}}\left(\tanh \frac{E_{k}-\mu}{2 T}+\tanh \frac{E_{k}+\mu}{2 T}\right)\right] .
\end{aligned}
$$

with the energies defined as

$$
\begin{aligned}
E_{\pi, k} & =\sqrt{k^{2}+2 U_{k, \sigma}}, \\
E_{\sigma, k} & =\sqrt{k^{2}+2 U_{k, \sigma}+4 \sigma^{2} U_{k, \sigma \sigma}}, \\
\tilde{E}_{\Delta, 0, k} & =\sqrt{k^{2}+2 U_{k, \Delta}}, \\
\tilde{E}_{\Delta, k} & =\sqrt{k^{2}+2 U_{k, \Delta}+4 \Delta^{2} U_{k, \Delta \Delta}}, \\
E_{\Delta, 0} & =\sqrt{\tilde{E}_{\Delta, 0, k}^{2}-4 \mu^{2}} \\
E_{\Delta} & =\sqrt{\tilde{E}_{\Delta, k}^{2}-4 \mu^{2}}, \\
E_{k} & =\sqrt{k^{2}+\left(h_{\phi} \sigma\right)^{2}} \\
E_{k}^{ \pm} & =\sqrt{\left(E_{k} \pm \mu\right)^{2}+\left(h_{\Delta} \Delta\right)^{2}},
\end{aligned}
$$

where we introduced the short-hand notation

$$
\begin{array}{ll}
U_{k, \sigma}=\partial_{\sigma} U_{k}\left(\sigma^{2}, \Delta^{2}\right), & U_{k, \sigma \sigma}=\partial_{\sigma \sigma} U_{k}\left(\sigma^{2}, \Delta^{2}\right), \\
U_{k, \Delta}=\partial_{\Delta} U_{k}\left(\sigma^{2}, \Delta^{2}\right), & U_{k, \Delta \Delta}=\partial_{\Delta \Delta} U_{k}\left(\sigma^{2}, \Delta^{2}\right) .
\end{array}
$$

The $z_{i}$ are defined as:

$$
\begin{aligned}
z_{k+1} & =-\frac{i}{3}\left(\beta_{2}+\eta^{k} C+\frac{b_{0}}{\eta^{k} C}\right), \quad k \in\{0,1,2\}, \\
z_{4} & =z_{1}, z_{5}=z_{2}, z_{6}=z_{3},
\end{aligned}
$$


where

$$
\begin{aligned}
& C=\sqrt[3]{\frac{b_{1}+\sqrt{b_{1}^{2}-4 b_{0}^{3}}}{2}}, \\
& \eta=\frac{-1+\sqrt{-3}}{2},
\end{aligned}
$$

and

$$
\begin{aligned}
& b_{0}=\beta_{2}^{2}-3 \beta_{1} \\
& b_{1}=2 \beta_{2}^{3}-9 \beta_{2} \beta_{1}+27 \beta_{0} .
\end{aligned}
$$

The coefficients $\alpha_{i}$ and $\beta_{i}$ are given by

$$
\begin{aligned}
& \alpha_{0}=E_{\Delta, k}^{2} E_{\Delta, 0, k}^{2}+E_{\Delta, k}^{2} E_{\sigma, k}^{2}+E_{\Delta, 0, k}^{2} E_{\sigma, k}^{2}-16 \sigma^{2} \Delta^{2} U_{k, \sigma \Delta}^{2}, \\
& \alpha_{1}=2\left(E_{\Delta, k}^{2}+E_{\Delta, 0, k}^{2}+E_{\sigma, k}^{2}+8 \mu^{2}\right), \\
& \beta_{0}=E_{\Delta, 0, k}^{2}\left(E_{\Delta, k}^{2} E_{\sigma, k}^{2}-\sigma^{2} \Delta^{2} U_{k, \sigma \Delta}^{2}\right), \\
& \beta_{1}=\alpha_{0}+16 E_{\sigma, k}^{2} \mu^{2} \\
& \beta_{2}=E_{\Delta, k}^{2}+E_{\Delta, 0, k}^{2}+E_{\sigma, k}^{2}+16 \mu^{2} .
\end{aligned}
$$

The diagrammatic representation of Eq. 4.32 is presented in Fig. 4.2. We have two different diquark energy functions because only the anti-blue (composed of red and green quarks) diquark comes with a diquark gap, while the other two have no energy gap. Furthermore, the tilde notation over the diquark energies highlights that these are not the true energies because their masses also depend on the quark chemical potential. We have defined $\tilde{E}_{k, \Delta, 0}$ and $\tilde{E}_{\Delta, k}$ such that they are analogous to $E_{\pi, k}$ and $E_{\sigma, k}$.

The first term in the first square bracket of Eq. (4.32) stems from the pion loop, the second term arises from the mixed $\sigma$ and gapped diquark loop and the third one from the ungapped diquark loop. The two terms in the second square bracket are contributions from the gapped and ungapped quarks, respectively.

It is instructive to try to derive the quark-meson model flow equation starting from the QMD model. The flow equation for the effective potential of the QM model is given by [36]

$$
\begin{aligned}
\partial_{k} U_{k}=\frac{k^{4}}{12 \pi^{2}} & {\left[\frac{3}{E_{\pi, k}} \operatorname{coth} \frac{E_{\pi, k}}{2 T}+\frac{1}{E_{\sigma, k}} \operatorname{coth} \frac{E_{\sigma, k}}{2 T}\right.} \\
& \left.-\frac{12}{E_{k}}\left(\tanh \frac{E_{k}-\mu}{2 T} \tanh \frac{E_{k}+\mu}{2 T}\right)\right] .
\end{aligned}
$$

First, let us try to reproduce the fermionic contribution by taking the limit 
$\Delta \rightarrow 0$ (which is equivalent to $h_{\Delta} \rightarrow 0$ ). The first term of the fermionic contribution in Eq. (4.32) reduces to

$$
\begin{gathered}
E_{k}^{ \pm} \stackrel{\Delta \rightarrow 0}{\longrightarrow}\left|E_{k} \pm \mu\right|=\operatorname{sgn}\left(E_{k} \pm \mu\right)\left(E_{k} \pm \mu\right) \\
\sum_{ \pm} \frac{1 \pm \frac{\mu}{E_{k}}}{E_{k}^{ \pm}} \tanh \frac{E_{k}^{ \pm}}{2 T} \stackrel{\Delta \rightarrow 0}{\longrightarrow} \sum_{ \pm} \frac{E_{k} \pm \mu}{E_{k} \operatorname{sgn}\left(E_{k} \pm \mu\right)\left(E_{k} \pm \mu\right)} \tanh \frac{\operatorname{sgn}\left(E_{k} \pm \mu\right)\left(E_{k} \pm \mu\right)}{2 T} \\
\quad=\frac{1}{E_{k}}\left(\tanh \frac{E_{k}-\mu}{2 T}+\tanh \frac{E_{k}+\mu}{2 T}\right)
\end{gathered}
$$

The gapped fermion contribution therefore simply reduces to the ungapped one, restoring the fermionic part of the QM-model flow equation. However, the bosonic contribution does not reduce to the QM model, which is seen when looking at the second and third terms of the first square bracket in Eq. (4.32). Both of these terms include the diquark energies with and without diquark background field and in the $\Delta \rightarrow 0$ limit the diquark energy with background field becomes $\tilde{E}_{\Delta, k} \stackrel{\Delta \rightarrow 0}{\longrightarrow} \tilde{E}_{\Delta, 0, k}$ in the zero diquark gap limit. Even if we assumed that $U_{k, \Delta}=0$ the diquark contributions would not vanish, because then $\tilde{E}_{\Delta, 0, k}=k$, i.e., diquarks behave as massless particles. This discussion might seem trivial because it is clear that the diquark fluctuations should contribute to the effective potential even in the $\Delta \rightarrow 0$ limit. But here we would highlight that this is in contrast to the MFA. In the previous chapter we also performed the $\Delta \rightarrow 0$ limit for the grand potential (3.24) but there we exactly reproduced the grand potential of the NJL model without diquarks. Later on, when we discuss the results, we will confirm, that in the zero-diquark gap limit we will obtain slightly different results for the QMD model compared to the QM model, although the difference is very small.

The goal of solving equation (4.54) is to obtain the effective potential in the IR, i.e., $U_{k \rightarrow 0}$. We can then derive the grand potential

$$
\Omega(T, \mu)=U_{k \rightarrow 0} .
$$

From Eq. (4.58) important thermodynamic quantities can be derived using the standard definitions from statistical physics. For the pressure, entropy density, and quark number density these are

$$
\begin{aligned}
p(T, \mu) & =-\Omega(T, \mu)+\Omega(0,0), \\
s(T, \mu) & =\frac{\partial p(T, \mu)}{\partial T}, \\
n(T, \mu) & =\frac{\partial p(T, \mu)}{\partial \mu} .
\end{aligned}
$$

Of particluar interest will be the entropy density. In a recent study [1] it 
has been shown that for a specific choice of the UV potential negative entropy densities are found for small temperatures in the region of the first-order phase transition line. In this thesis, we would like to address this problem by investigating how the negative entropy-density region is affected when diquark degrees of freedom are included. Another possibility for the emergence of the negative entropy-density region is that the assumption of spatially homogeneous phases leads to a thermodynamic instability in the FRG formalism. In this thesis we follow the approach of Ref. [1], where a stability analysis of the homogeneous phase in the FRG formalism has been conducted, and apply this method to the QMD model. This method will be discussed in the following section.

\subsection{Flow of the Two-Point Function and Stability Analysis}

As already mentioned, the flow equations for the two-point functions can be obtained by repeated functional differentiation, see also Eq. (E.8). In general, the flow equation for an $n$-point function depends on the flow equations for the $(n+1)$ - and $(n+2)$-point functions, leading to an infinite tower of coupled flow equations. Therefore, the right-hand side of the flow equation for the two-point function then depends on the three- and four-point functions. Here we follow Ref. [96] and simply neglect all $n$-point function for $n>4$. In addition, we neglect the momentum dependence of the three- and four-point functions. In this work we will focus on the pion two-point function, which is defined as

$$
\begin{aligned}
\partial_{k} \Gamma_{k, \pi}^{(2)}(p)= & \operatorname{Tr}\left[\partial_{k} R_{k}(q+p) G_{k, \mathrm{~B}}(q+p) \Gamma_{k, \pi}^{(3)} G_{k, \mathrm{~B}}(q) \Gamma_{k, \pi}^{(3)} G_{k, \mathrm{~B}}(q+p)\right] \\
& -\frac{1}{2} \operatorname{Tr}\left[G_{k, \mathrm{~B}}(q) \Gamma_{k, \pi \pi}^{(4)} G_{k, \mathrm{~B}}(q)\right], \\
& -2 \operatorname{Tr}\left[\partial_{k} R_{k}(q+p) G_{k, \psi}(q+p) \Gamma_{k, \bar{\psi} \psi \pi}^{(2,1)} G_{k, \psi}(q) \Gamma_{k, \bar{\psi} \psi \pi}^{(2,1)} G_{k, \psi}(q+p)\right] .
\end{aligned}
$$

$G_{k, \mathrm{~B}}=\left(\Gamma_{k, \mathrm{~B}}^{(2)}+R_{k, \mathrm{~B}}\right)^{-1}$ is the bosonic FRG propagator in field space and $G_{k, \mathrm{~F}}=\left(\Gamma_{k, \mathrm{~F}}^{(2)}+R_{k, \mathrm{~F}}\right)^{-1}$ the fermionic one. The vertex functions are simply $\Gamma_{k, \pi}^{(3)}=\frac{\delta}{\delta \pi} \Gamma_{k, \mathrm{~B}}^{(2)} \Gamma_{k, \pi}^{(4)}=\frac{\delta}{\delta \pi} \Gamma_{k, \pi}^{(3)}$ and $\Gamma_{k, \bar{\psi} \psi \pi}^{(2,1)}=\frac{\delta}{\delta \pi} \Gamma_{k, \mathrm{~F}}^{(2)} \cdot q$ is the internal momentum, which is integrated over in the trace, and $p$ the external momentum. More explicit expressions for the QM and QMD model are given in App. E. Unlike the flow equation for $U_{k}$, Eq. (4.62) is an ordinary differential equation because we always evaluate it at the minimum position of the effective potential. Since Eq. (4.62) explicitly depends on $U_{k}^{(n)}(n \leq 4)$ we need to first solve the partial differential equation in Eq. (4.32) and save it at $N_{k}$ steps during the flow. In this thesis we choose $N_{k}=100$. We then interpolate the effective potential between the saved flow-time steps and subsequently 
we can feed the interpolated effective potential and its derivatives into Eq. (4.62).

The two-point function is a very important quantity because several observables such as spectral functions and transport coefficients can be derived from it. In this thesis we will focus on the stability analysis of the homogeneous phase, for which the retarded two-point function at vanishing external frequency will be investigated. The initial condition at the UV scale reads:

$$
\Gamma_{\Lambda, \pi \pi}^{(2)}(0, \vec{p})=-\vec{p}^{2}-2 \partial_{\phi^{2}} U_{\Lambda}\left(\phi^{2}, \Delta_{a}^{2}\right) .
$$

In general, a stability analysis means to examine the roots of the inverse propagator, i.e., $D^{-1}(\omega,|\vec{p}|)=0$, which signals the formation of a physical excitation. A stability analysis of the homogeneous phase is then defined by $D^{-1}(\omega=0,|\vec{p}|)=0$, since this is the necessary condition for a resonance to be added into the ground state without cost of energy, i.e., condensation. Taking the well-known pion p-wave condensation in nuclear matter [16, 17] as an example, the necessary condition corresponds to:

$$
D_{\pi}^{(-1)}(\omega=0,|\vec{p}|)=-\vec{p}^{2}-m_{\pi}^{2}-\Pi(\omega=0,|\vec{p}|)=0,
$$

where $\Pi(\omega=0,|\vec{p}|)$ is the pion self-energy in medium. In vacuum the selfenergy is zero and therefore the inverse pion propagator is inevitably negative. However, in medium the self-energy might become negative and for some critical density it could produce an instability of the pion propagator. Precisely this was found in Ref. [16] in the case of pion p-wave interactions in symmetric nuclear matter.

Here we would like to perform a similar analysis in the FRG framework. To this end, we solve Eq. (4.62) for $\omega=0$ and subsequently, analogous to the pion-wave condensation example, we will look for the zeros of $\Gamma_{k, \pi}^{(2)}(\omega=$ $0,|\vec{p}|)=0$. We scan the phase diagram for these zero-crossings by solving Eq. (4.62) not only for each $(T, \mu)$ pair, but also for several values of $|\vec{p}|$. However, interpreting the instability as the formation of an inhomogeneous condensate is not as straightforward as in the case of pion p-wave condensation. The obvious reason is the fact, that the effective average action can only be considered as physical in the infrared because of the regulator dependence during the flow. On the other hand, it could be that an instability occurs during the flow and then disappears afterwards. In this case, the interpretation would not be so obvious. At the scale where the instability occurs, one would have to modify the flow equation to account for the inhomogeneity. But then the further flow would change afterwards, so that no definite statements are possible. We will come back to this issue when discussing the results of our stability analysis. 


\section{Chapter 5}

\section{Numerical Solution Details}

In Refs. [97, 98, 99, 100, 101] the issue has been raised that standard techniques to solve the flow equations for the effective potential might be unsuitable to guarantee the convergence to the correct solution of the corresponding partial differential equation. The method in question is the finitedifference method, which transforms the original partial differential equation into a system of $N$ coupled ordinary differential equations by expressing the field dependence on a discrete set of grid points in field space.

One issue with standard finite-difference methods is their inability to resolve discontinuous jumps in the potential, i.e., shock waves. If the model does not produce shock waves then the lack of the shock-capturing ability of the standard finite-difference method is not a serious problem, however it was found in Ref. [97] using the discontiuous Galerkin method that the $2+1$ dimensional $\mathrm{O}(N)$ model produces shock waves in the large- $N$ limit.

In order to ensure that the numerical results presented in this thesis are credible, we will use both the finite-difference method and the finite-volume method, which is able to resolve shocks reliably. In this way we can ensure that our results are independent of the numerical method.

In the finite-difference approach, one usually discretizes the effective potential in the chiral invariant $\phi_{0}^{2}=\sigma^{2}$, which leads to an effective potential that is defined on the positive domain as the invariant is manifestly positive. The discrete derivatives at the boundaries are then obtained by using forward finite differences at the origin and backward finite differences at $\sigma_{\max }^{2}$ thus no grid points outside of the domain of the effective potential are used.

For the finite-volume method the situation is different, since for the construction of the flux no finite differences are used. In order to reconstruct the potential and the flux at the origin, we need to introduce so-called ghost cells at the boundaries. These ghost cells are outside of the domain of the potential and if we express the effective potential in terms of its chiral invariant, this means that we need a ghost cell left to the boundary that is negative. This leads to a problem, as there are no negative $\sigma^{2}$ and therefore it is not clear what value we should assign to the potential at this ghost cell. One way to circumvent this problem in the finite-volume method is to discretize the effective potential with respect to $\sqrt{\phi_{0}^{2}}=\sigma$. The effective potential is then defined on the domain $\sigma \in[-\infty, \infty]$. We can still evaluate the effective potential in the positive domain and use the antisymmetry of $u=U_{\sigma}(\sigma) \equiv \partial_{\sigma} U(\sigma)$ 
in order to reconstruct the ghost cell left to the origin: $u\left(x_{1}\right)=-u\left(x_{-1}\right){ }^{1}$

Note that changing the field-dependence of the effective potential from $\rho=\sigma^{2}$ to $\sigma$ also changes the definition of the derivatives, i.e.,

$$
\begin{aligned}
m_{\pi}^{2} & =2 U_{\rho}(\rho)=u(\sigma) / \sigma \\
m_{\sigma}^{2} & =2 U_{\rho}(\rho)+4 \rho U_{\rho \rho}(\rho)=u_{\sigma}(\sigma), \\
U_{\rho}(\rho) & =\partial_{\rho} U(\rho), \quad U_{\rho \rho}(\rho)=\partial_{\rho \rho} U(\rho), \quad u_{\sigma}(\sigma)=\partial_{\sigma} u(\sigma) .
\end{aligned}
$$

In the next section we will outline the finite-volume method applied to the flow of equations in Eqs. (D.75) and (4.54) in more detail before discussing the numerical test. For some details on the finite-difference method as usually applied in FRG studies, we refer to Refs. [67, 69].

\subsection{Finite-Volume Method}

In this thesis we apply the high-resolution central scheme for convection-diffusion equations developed by Kurganov and Tadmor in Ref. [102] and further improved in Refs. [103, 104]. In order to apply this scheme the onedimensional partial differential equation in question needs to be cast into the following form:

$$
\partial_{t} u+\partial_{x} f(u)=\partial Q_{x}\left(u, u_{x}\right)
$$

$u=u(x, t)$ is the conserved quantity, $f(u)$ is the advection flux, and $Q_{x}\left(u, u_{x}\right)$ is the diffusion flux.

Let us first apply the KT scheme to the flow equation of the effective potential of the QM model given in Eq. (4.54). The corresponding flow equation can be separated into three different contributions (in the following we suppress the index denoting the scale dependence of the effective potential):

$$
\begin{aligned}
\partial_{k} U & =f\left(U_{\sigma}\right)+Q\left(U_{\sigma}, U_{\sigma \sigma}, \sigma\right)+s(\sigma), \\
f\left(U_{\sigma}\right) & =\frac{k^{4}}{12 \pi^{2}} \frac{3}{E_{\pi, k}} \operatorname{coth} \frac{E_{\pi, k}}{2 T}, \\
Q\left(U_{\sigma}, U_{\sigma \sigma}, \sigma\right) & =\frac{k^{4}}{12 \pi^{2}} \frac{1}{E_{\sigma, k}} \operatorname{coth} \frac{E_{\sigma, k}}{2 T}, \\
s(\sigma) & =\frac{k^{4}}{\pi^{2}} \frac{1}{E_{k}}\left(\tanh \frac{E_{k}-\mu}{2 T}+\tanh \frac{E_{k}+\mu}{2 T}\right) .
\end{aligned}
$$

\footnotetext{
${ }^{1}$ It should be noted however, that we performed some numerical tests where we naively constructed the left ghost cell with linear extrapolation. In these experiments we obtain the same potential in the infrared and the error scaling is the same as well. Although we should use the method described here, in practice it seems to make no difference.
} 
Next, we take the derivative of the flow equation with respect to $\sigma$ and introduce the new variable $u=\partial_{\sigma} U(\sigma)$.

$$
\partial_{k} u=\partial_{\sigma} f(u)+\partial_{\sigma} Q\left(u, u_{\sigma}, \sigma\right)+\partial_{\sigma} s(\sigma) .
$$

In this form the KT scheme can be applied. However, instead of solving the PDE for the effective potential directly, we can only solve this PDE for the derivative of the effective potential. The effective potential up to a constant can then be restored by integrating the solution of Eq. (5.9), i.e., $U=\int_{\sigma} u$.

A pedagogical python implementation of the KT Scheme applied to the quark-meson model can be found in App. F.2.

Next, we will look at the flow equation for the effective potential of the quark-meson-diquark model. In order to apply the two-dimensional KT scheme, the flow equation needs to be recast into the following form:

$$
\partial_{t} u+\partial_{x} f(u)+\partial_{y} g(u)=\partial_{x} Q_{x}\left(u, u_{x}, u_{y}\right)+\partial_{y} Q_{y}\left(u, u_{x}, u_{y}\right)
$$

$u=\left(u_{1}(x, y, t), u_{2}(x, y, t)\right)$ is a two-dimensional vector of conserved quantities, $f(u)=\left(f_{1}(u), f_{2}(u)\right)$ and $g(u)=\left(g_{1}(u), g_{2}(u)\right)$ are the advection fluxes, and

$$
\begin{aligned}
& Q_{x}\left(u, u_{x}, u_{y}\right)=\left(Q_{1, x}\left(u, u_{x}, u_{y}\right), Q_{2, x}\left(u, u_{x}, u_{y}\right)\right), \\
& Q_{y}\left(u, u_{x}, u_{y}\right)=\left(Q_{1, y}\left(u, u_{x}, u_{y}\right), Q_{2, y}\left(u, u_{x}, u_{y}\right)\right),
\end{aligned}
$$

are the diffusion fluxes.

The flow equation (4.32) depends on two fluxes in the $\sigma$ and $\Delta$ directions, as well as the diffusion flux and a source term:

$$
\begin{aligned}
& \partial_{k} U=f\left(U_{\sigma}\right)+g\left(U_{\Delta}\right)+Q\left(U_{\sigma}, U_{\sigma \sigma}, U_{\Delta}, U_{\Delta \Delta}, U_{\sigma \Delta}, \sigma, \Delta\right)+s(\sigma, \Delta) \\
& f\left(U_{\sigma}\right)=\frac{k^{4}}{12 \pi^{2}} \frac{3}{E_{k, \pi}} \operatorname{coth} \frac{E_{\pi, k}}{2 T}, \\
& g\left(U_{\Delta}\right)=\frac{k^{4}}{12 \pi^{2}} \frac{2}{\tilde{E}_{\Delta, 0, k}}\left(\operatorname{coth} \frac{\tilde{E}_{\Delta, 0, k}-2 \mu}{2 T}+\operatorname{coth} \frac{\tilde{E}_{\Delta, 0, k}+2 \mu}{2 T}\right) \\
& Q\left(U_{\sigma}, U_{\sigma \sigma}, U_{\Delta}, U_{\Delta \Delta}, U_{\sigma \Delta}, \sigma, \Delta\right)=\frac{k^{4}}{12 \pi^{2}} \sum_{i=1}^{3} \frac{\alpha_{0}-\alpha_{1} z_{i}^{2}+3 z_{i}^{4}}{\left(z_{i+1}^{2}-z_{i}^{2}\right)\left(z_{i+1}^{2}-z_{i}^{2}\right) z_{i}} \operatorname{coth} \frac{z_{i}}{2 T} \\
& s(\sigma, \Delta)=\frac{k^{4}}{3 \pi^{2}}\left[2 \sum_{ \pm} \frac{1 \pm \frac{\mu}{E_{k}}}{\left(E_{k}^{ \pm}\right)^{2}} \tanh \frac{E_{k}^{ \pm}}{2 T}+\frac{1}{E_{k}}\left(\tanh \frac{E_{k}-\mu}{2 T}+\tanh \frac{E_{k}+\mu}{2 T}\right)\right]
\end{aligned}
$$


In order to bring it in the form of Eq. (5.10), we take the derivatives with respect to $\sigma$ and $\Delta$ and thus obtain two partial differential equations:

$$
\begin{aligned}
& \partial_{k} \partial_{\sigma} U=\partial_{\sigma} f\left(U_{\sigma}\right)+\partial_{\sigma} g\left(U_{\Delta}\right)+\partial_{\sigma} Q\left(U_{\sigma}, U_{\sigma \sigma}, U_{\Delta}, U_{\Delta \Delta}, U_{\sigma \Delta}, \sigma, \Delta\right)+\partial_{\sigma} s(\sigma, \Delta) \\
& \partial_{k} \partial_{\Delta} U=\partial_{\Delta} f\left(U_{\sigma}\right)+\partial_{\Delta} g\left(U_{\Delta}\right)+\partial_{\Delta} Q\left(U_{\sigma}, U_{\sigma \sigma}, U_{\Delta}, U_{\Delta \Delta}, U_{\sigma \Delta}, \sigma, \Delta\right)+\partial_{\Delta} s(\sigma, \Delta) .
\end{aligned}
$$

Then, we define $V=\partial_{\sigma} U$ and $W=\partial_{\Delta} U$, yielding

$$
\begin{aligned}
\partial_{k} V & =\partial_{\sigma} f(V)+\partial_{\sigma} g(W)+\partial_{\sigma} Q\left(V, V_{\sigma}, W, W_{\Delta}, V_{\Delta}, \sigma, \Delta\right)+\partial_{\sigma} s(\sigma, \Delta) \\
\partial_{k} W= & \partial_{\Delta} f(V)+\partial_{\Delta} g(W)+\partial_{\Delta} Q\left(V, V_{\sigma}, W, W_{\Delta}, V_{\Delta,} \sigma, \Delta\right)+\partial_{\Delta} s(\sigma, \Delta) \\
\partial_{k}\left(\begin{array}{c}
V \\
W
\end{array}\right)= & \partial_{\sigma}\left(\begin{array}{c}
f+g \\
0
\end{array}\right)+\partial_{\Delta}\left(\begin{array}{c}
0 \\
f+g
\end{array}\right)+\partial_{\sigma}\left(\begin{array}{c}
Q \\
0
\end{array}\right)+\partial_{\Delta}\left(\begin{array}{c}
0 \\
Q
\end{array}\right) \\
& +\partial_{\sigma}\left(\begin{array}{c}
s \\
0
\end{array}\right)+\partial_{\Delta}\left(\begin{array}{l}
0 \\
s
\end{array}\right)
\end{aligned}
$$

If we were to take only the derivative with respect to either to $\sigma$ or $\Delta$ we would not be able to recover the full information of the effective potential $U_{k}$. For instance, if we have the function $f(x, y)=x^{2}+y^{2}+x y+$ const., upon taking the derivative in one direction, e.g., $\partial_{x} f(x, y)=2 x+y$, the information for summands that purely depend on $y$ would be lost. On the other hand, if we have the solutions for both $\partial_{\sigma} U=V$ and $\partial_{\Delta} U=W$ we can restore the full information of the effective potential (except for an irrelevant constant term) in the following way:

$$
\begin{aligned}
U_{1}(\sigma, \Delta) & =\int_{0}^{\sigma} \mathrm{d} \sigma^{\prime} V\left(\sigma^{\prime}, \Delta\right), \quad U_{2}(\sigma, \Delta)=\int_{0}^{\Delta} \mathrm{d} \Delta^{\prime} W\left(\sigma, \Delta^{\prime}\right), \\
\rightarrow U(\sigma, \Delta) & =U_{1}(\sigma, \Delta=0)+U_{2}(\sigma, \Delta)=U_{1}(\sigma, \Delta)+U_{2}(\sigma=0, \Delta) .
\end{aligned}
$$

Because of the symmetry of second derivatives, i.e., $\partial_{\sigma} \partial_{\Delta} f=\partial_{\Delta} \partial_{\sigma} f$, the described procedure also yields a constraint that needs to be fulfilled when solving the PDE in Eq. (5.21) numerically, namely $\partial_{\Delta} V=\partial_{\sigma} W \equiv U_{\sigma \Delta}$. This serves as a useful check when implementing the PDE numerically.

\subsection{Numerical Tests}

In the following we test the reliability of the standard finite-difference and the KT finite-volume method in the low-temperature regime of the phase diagram by employing two different numerical tests. The first one is to determine the dependence of various IR observables on the number of grid points 
with the grid length held fixed. We call this test the grid point test. The second one consists of varying the grid length while keeping the distance between two grid points fixed. This test will be called grid-length test.

All numerical tests are performed for both the quark-meson model and the quark-meson-diquark model. In the QM model we plot the effective potential at the minimum, the chiral condensate, and the pion and sigma masses. In the QMD model we additionally plot the diquark condensate and the diquark mass. We determine the minimum of the effective potential by first interpolating the effective potential obtained in the IR with both methods using quartic splines and then applying a numerical minimization procedure. We focus on three different points in the phase diagram, one in the vacuum, one close to the chiral first-order phase transition and one for larger chemical potential beyond the chiral first-order phase transition.

In the following figures the black solid lines correspond to the test results obtained by using the FDM, while the blue dashed lines are obtained by using the FVM. When we speak of relative errors, we mean $O_{\mathrm{FDM}} / O_{\mathrm{FVM}}-1$, where $O_{\mathrm{FDM}}$ denotes the observable $O$ obtained using the FDM and $O_{\mathrm{FVM}}$ the observable $O$ obtained using the FVM.

\subsubsection{Numerical Tests: Quark-Meson Model}

For the gridpoint test we fix the extent of the grid to $\sigma_{\max }^{2}=180^{2} \mathrm{MeV}^{2}$, while for the grid-length test we fix the distance between two grid points to $d \sigma^{2}=140^{2} / 60 \mathrm{MeV}^{2}$. As a reminder, for the FDM we use discretization in terms of $\rho=\sigma^{2}$ with length $\sigma_{\max }^{2}$ and for the FVM we use the discretization in terms of $\sigma$ with length $\sigma_{\max }$.

The UV potential of the QM model reads:

$$
U_{\Lambda, \mathrm{QM}}=\frac{1}{2} m_{\sigma}^{2} \sigma^{2}+\frac{1}{4} \lambda_{\sigma} \sigma^{4}
$$

The parameter set for the QM-model numerical tests are listed in Tab. 5.1, which are the same as in Ref. [60].

\begin{tabular}{|c|c|c|c|c|c|}
\hline$\Lambda$ & $m_{\sigma} / \Lambda$ & $\lambda_{\sigma}$ & $c / \Lambda^{3}$ & $h_{\phi}$ & $k_{\mathrm{IR}}$ \\
\hline $1 \mathrm{GeV}$ & 0.969 & 0.001 & 0.00175 & 4.2 & $50 \mathrm{MeV}$ \\
\hline
\end{tabular}

TABLE 5.1: Parameters for the QM-model numerical tests.

In Figs. 5.1-5.4 we plot the various observables of the QM model in dependence on the number of grid points. The smallest number of grid points is chosen to be $\#=15$. For a smaller number of grid points imaginary energies occur during the flow since the derivatives of the effective potentials are becoming too imprecise. In Fig. 5.1 the vacuum results are shown and we find by looking at the abscissa that the observables barely change. The relative errors are below $1 \%$, except for the sigma mass, where it fluctuates with about $5 \%$ relative variance for very few grid points. At about 50 grid points 
the FDM and the FVM start to agree very well and for the largest number of grid points the difference between methods is significantly smaller than $1 \%$.

The result in the vicinity of the chiral first-order phase transition is presented in Fig. 5.2. Here, the grid-point dependence becomes more significant. For instance, for the chiral order parameter, cf. upper right panel, we note that for less than 30 grid points the wrong chiral order parameter of the chirally broken phase is found when using the FDM. The FVM on the other hand seems to yield the correct position of the minimum even for a smaller number of grid points. This might be attributed to the fact that for the FVM we use the $\sigma$-grid instead of the $\rho$-grid. Accordingly, in the lower panel, we find the correct pion and sigma masses also at about 30 grid points for the FDM as the masses depend on the position of the minimum. For an increasing number of grid points both the FDM and the FVM again agree very well. It is also instructive to have a look at Fig. 5.3 where the full effective potentials in dependence on the number of grid points are presented.

In Fig. 5.4 we present the case for $\mu=400 \mathrm{MeV}$. Again we find a good agreement between the FDM and the FVM, however the discrepancy between both methods becomes larger. For instance the relative error for the effective potential at the minimum is about 3\%. https:/ / www.overleaf.com/project/5eac252ad
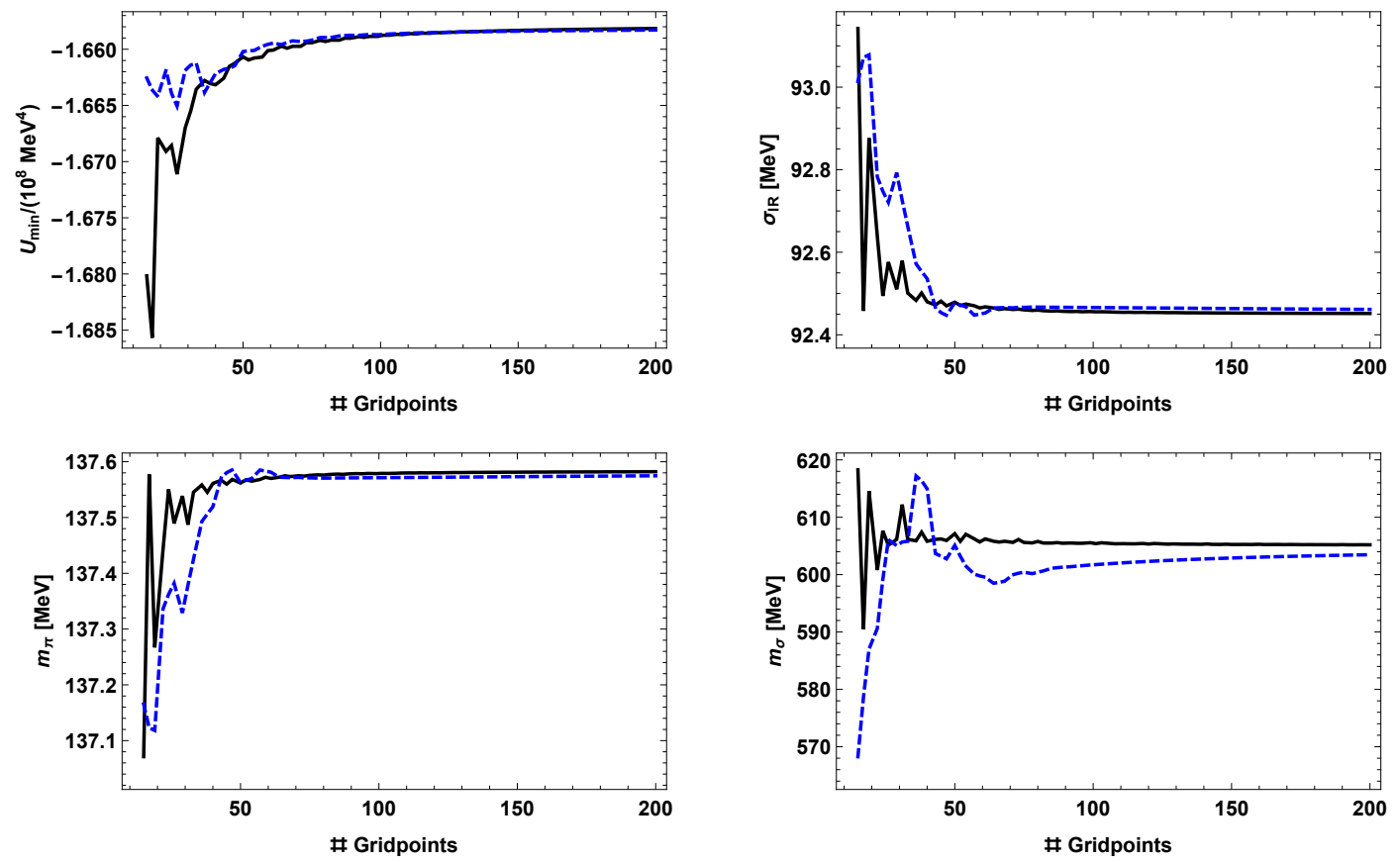

FIGURE 5.1: Black: FDM, blue-dashed: FVM. Various IR observables are plotted with respect to the number of grid points. Here $T=5 \mathrm{MeV}$ and $\mu=0 \mathrm{MeV}$. The relative errors for each grid configuration are less than $1 \%$.

Next, we will vary the grid length while keeping the grid spacing constant. This allows us to investigate the influence of the boundaries on the flow of the effective potential. In all plots of Figs. 5.5-5.7 we see that the dependence on the extent of the grid decreases rapidly for increasing length. Interestingly, for very small lengths the FVM on the linear $\sigma$ grid depends very strongly on the grid size while for the FDM even at very small grid 

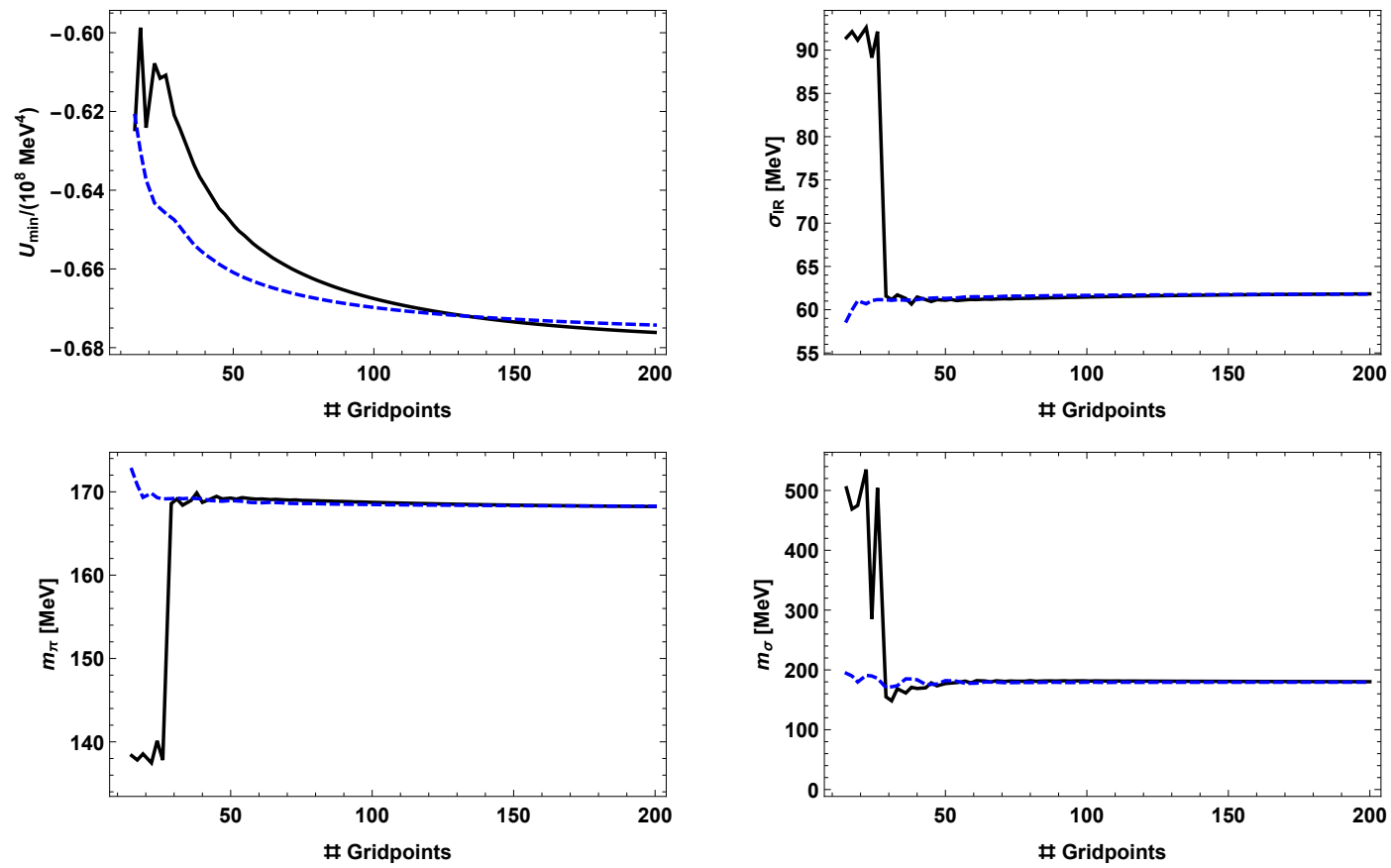

FIGURE 5.2: Black: FDM, blue-dashed: FVM. Plot of the grid-point dependence close to the first-order phase transition line at $T=5 \mathrm{MeV}$ and $\mu=330 \mathrm{MeV}$. Here the FDM yields the wrong minimum for the number of gridpoints $\#<30$.
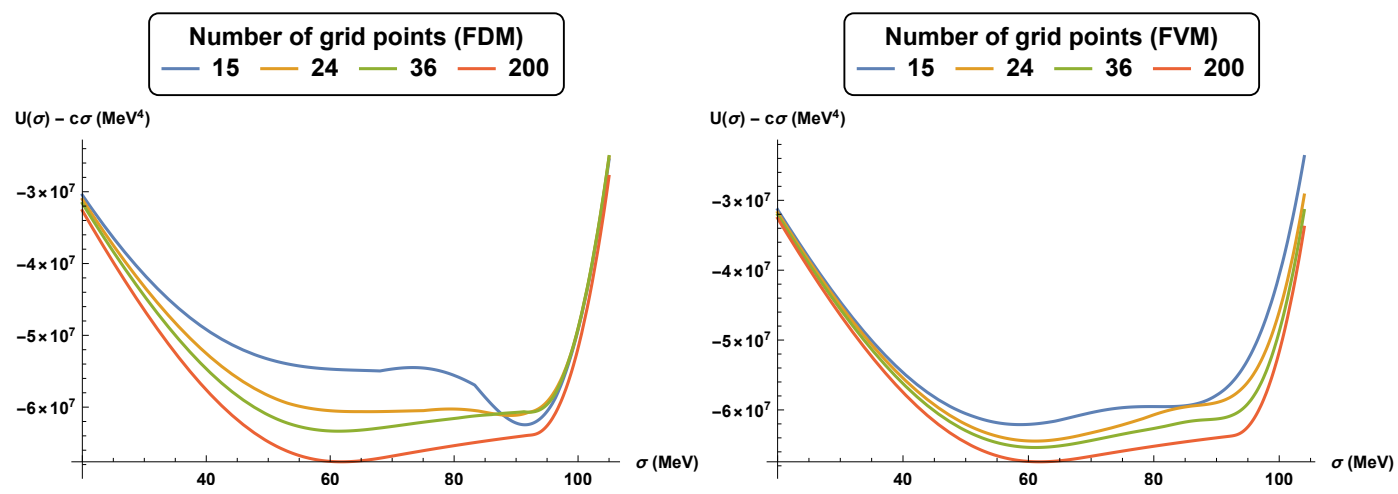

FIGURE 5.3: The FDM and FVM grid-point dependence of the full potential close to the first-order phase transition line at $T=5 \mathrm{MeV}$ and $\mu=330 \mathrm{MeV}$. In the case of the FDM, for a small number of grid points the solution of the flow equation converges to a wrong solution, where the location of the minimum is still in the chirally broken phase. On the other hand, for the FVM even for very few grid points the correct minimum is obtained. 

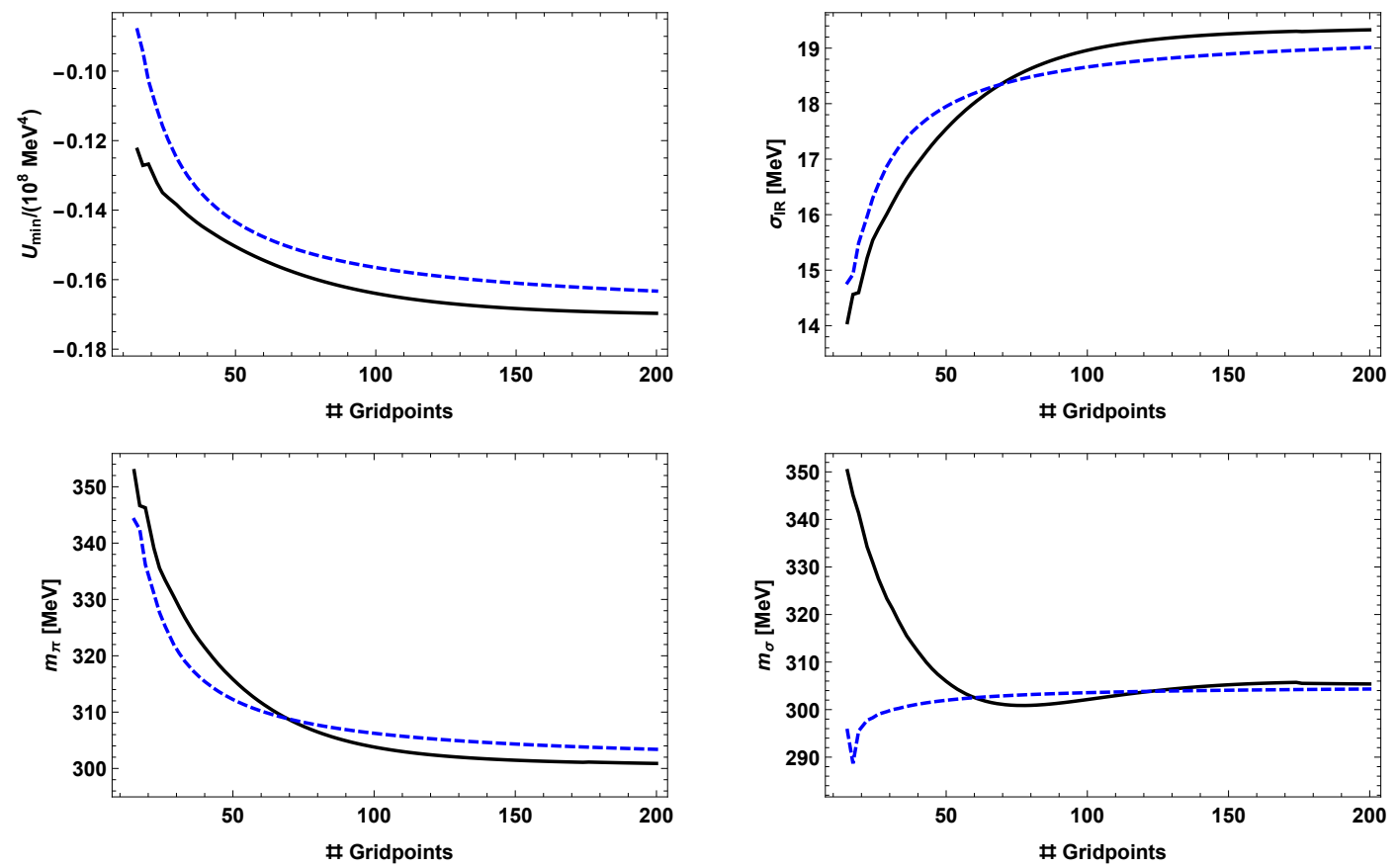

FIGURE 5.4: Black: FDM, blue-dashed: FVM. Plot of the grid-point dependence at $T=5 \mathrm{MeV}$ and $\mu=400 \mathrm{MeV}$.

lengths reasonable results are obtained. The grid-size dependence is most critical in Fig. 5.6, where only for grid sizes larger than $\sim 160 \mathrm{MeV}$ both the FVM and the FDM coincide.
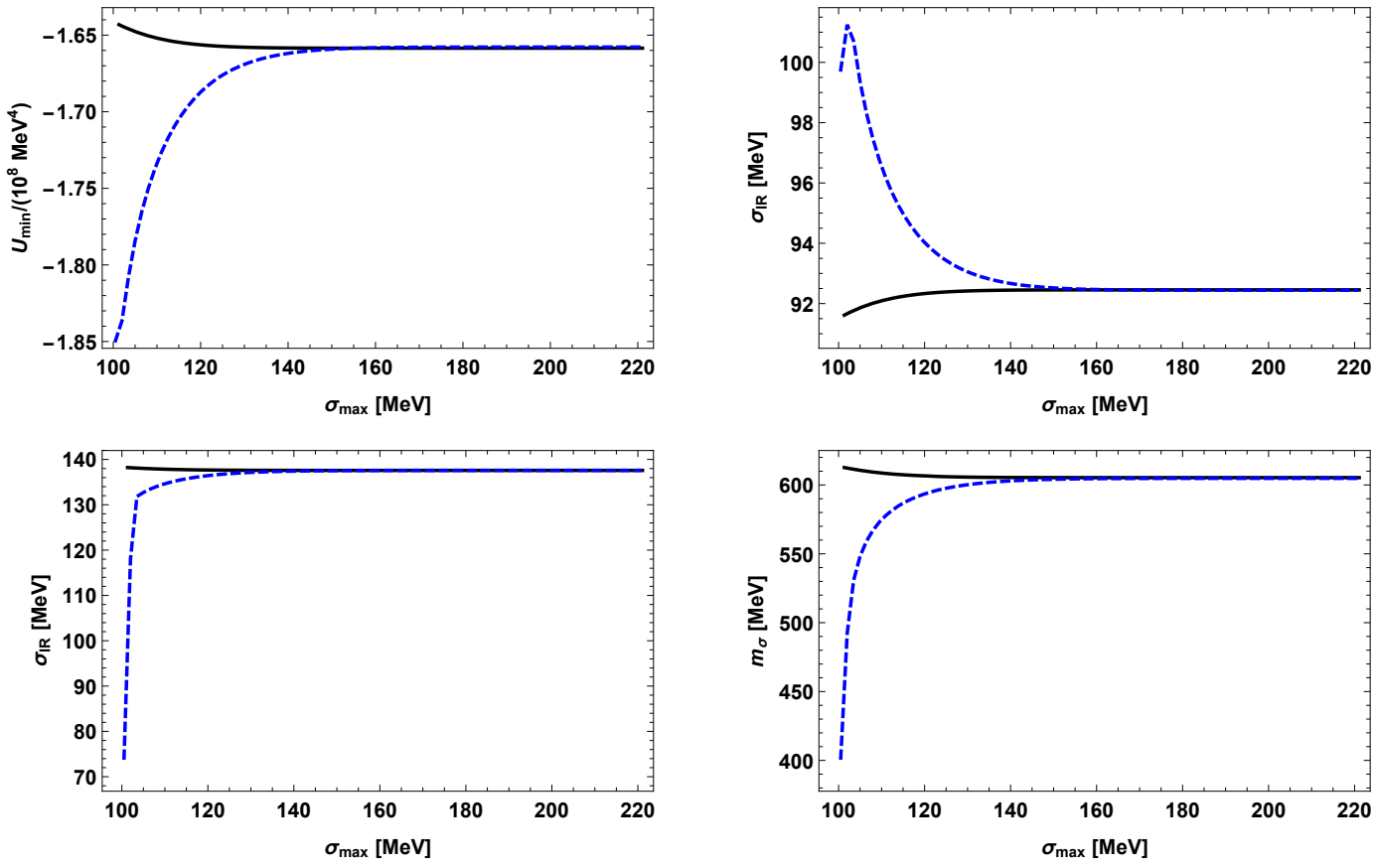

FIGURE 5.5: Black: FDM, blue-dashed: FVM. Various IR observables are plotted with varying grid extent and fixed $d \sigma^{2}=140^{2} / 60 \mathrm{MeV}^{2}$. Here $T=5 \mathrm{MeV}$ and $\mu=0 \mathrm{MeV}$. The relative errors for each grid configuration are less than $1 \%$. 

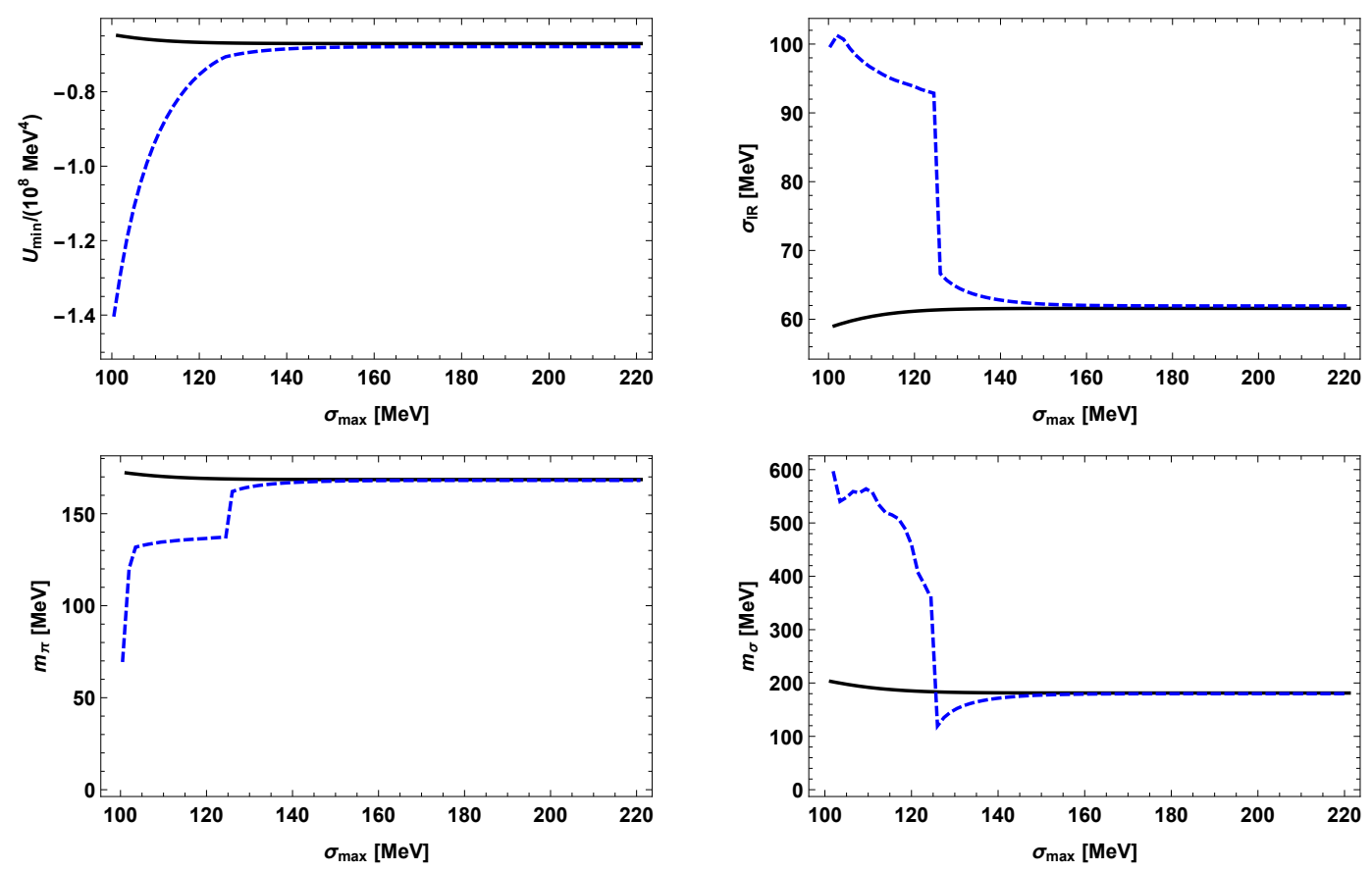

FIGURE 5.6: Black: FDM, blue-dashed: FVM. Plot of the grid-length dependence close to the first-order phase transition line at $T=5 \mathrm{MeV}$ and $\mu=330 \mathrm{MeV}$. Here the results become more uncertain, however we can observe convergent behavior.
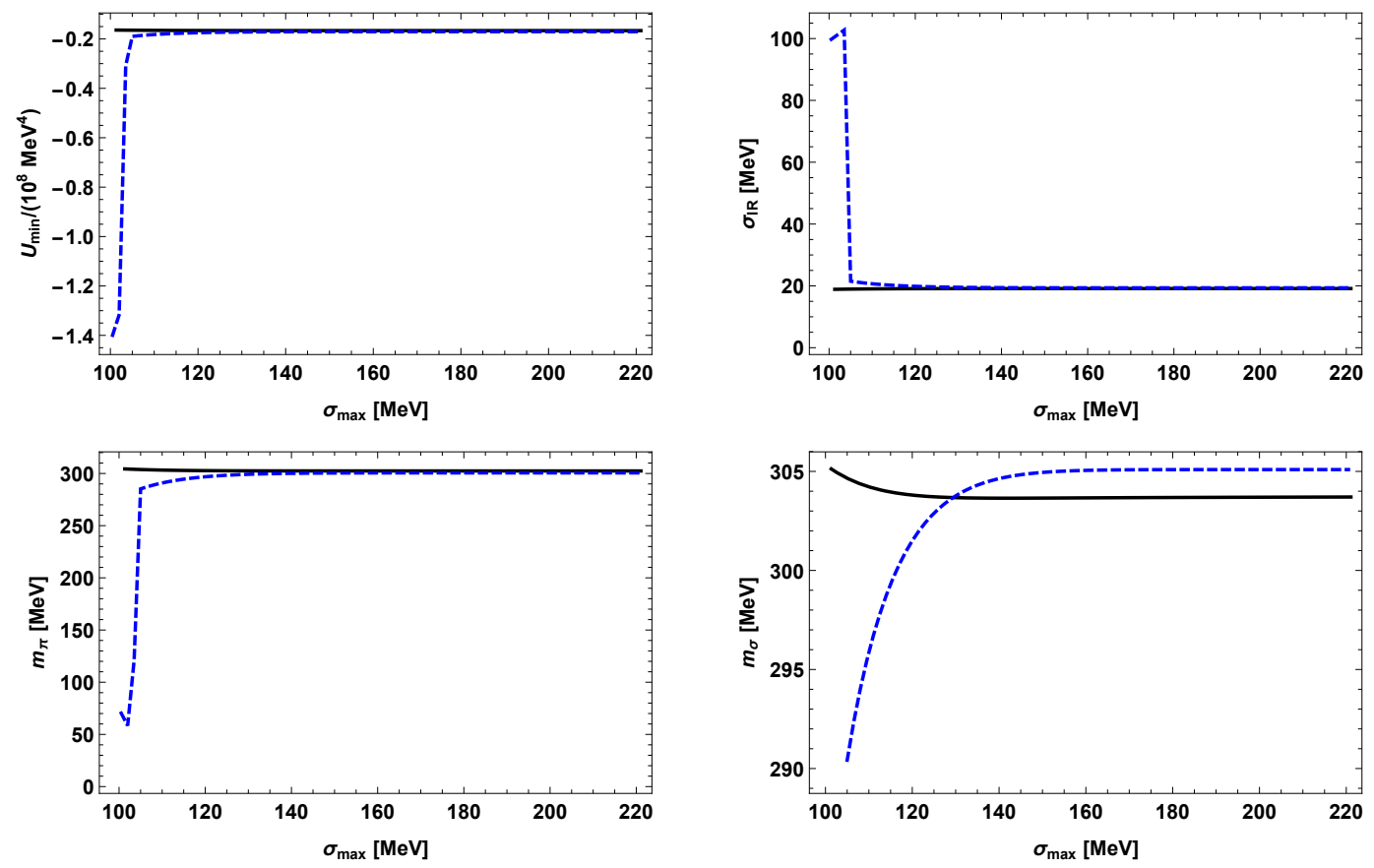

FIGURE 5.7: Black: FDM, blue-dashed: FVM. Plot of the grid-length dependence at $T=5 \mathrm{MeV}$ and $\mu=400 \mathrm{MeV}$. Both methods approximately converge to the same values for increasing grid extent. 


\subsubsection{Numerical Tests: Quark-Meson-Diquark Model}

In the following, we will perform the same numerical tests as in the previous subsection, but this time to compare the 2D finite-difference method and the 2D finite-volume method. The parameters of the UV potential are listed in Tab. 5.2 with the UV potential given as

$$
U_{\Lambda, \mathrm{QMD}}=\frac{1}{2} m_{\sigma}^{2} \sigma^{2}+\frac{1}{2} m_{\Delta}^{2} \Delta^{2}+\frac{1}{4} \lambda_{\sigma} \sigma^{4}+\frac{1}{4} \lambda_{\Delta} \Delta^{4}+\frac{1}{4} \lambda_{\sigma \Delta} \sigma^{2} \Delta^{2}
$$

\begin{tabular}{|c|c|c|c|c|c|c|c|c|c|}
\hline$\Lambda$ & $m_{\sigma} / \Lambda$ & $\lambda_{\sigma}$ & $m_{\Delta} / \Lambda$ & $\lambda_{\Delta}$ & $\lambda_{\sigma \Delta}$ & $c / \Lambda^{3}$ & $h_{\phi}$ & $h_{\Delta}$ & $k_{\mathrm{IR}}$ \\
\hline $1 \mathrm{GeV}$ & 0.969 & 0.001 & 0.9 & 8 & -0.6 & 0.00175 & 4.2 & 3 & $80 \mathrm{MeV}$ \\
\hline
\end{tabular}

TABLE 5.2: Employed parameters for the QMD model.

In Figs. 5.8-5.10 we plot various IR observables such as the position of the minimum, the effective potential at the minimum (neglecting a constant term), as well as the masses of the mesonic degrees of freedom, in dependence on the number of grid points in each direction. Note, that the diquark mass actually depends on the chemical potential but here we plot the diquark mass with background field neglecting the contribution from the chemical potential such that we are left with only one mass definition, $m_{\Delta}=\sqrt{2 U_{\Delta}+4 \Delta^{2} U_{\Delta \Delta}}$. Also, note that the number of grid points is the same in both directions. For example, for 80 grid points in each dimension we have a total of $80 \times 80=6400$ grid points for the full 2D grid. Similar as for the test cases of the QM model we find that the FDM and FVM tend to approach a common limit, however the discrepancy is somewhat larger.

In Figs. 5.11-5.13 we present the results for varying grid lengths and constant grid spacing. Here we also find small relative errors between both the FDM and FVM for the largest grid extent with the notable exception of the $\Delta$ mass in Fig. 5.12, where the relative error is approximately $2.5 \%$.

The alert reader will have noticed that we used larger temperatures for the QMD-model test cases. This is because we were not able to solve the flow equation at smaller temperatures using the FV method. We observed that in the low-temperature region the characteristic velocities at the grid boundaries become extremely large and thus the time steps extremely small, even smaller than floating-point precision and thus the numeric flow freezes effectively. This issue might be related to the specific implementation of our code, which can be found in App. F.3. For the finite-difference method this problem does not arise for $T>5 \mathrm{MeV}$ and $\mu<450 \mathrm{MeV}$. Because with the FDM we are able to access lower temperatures at large $\mu$ the results presented in the next chapter are obtained by using the FDM, however, we have checked all results with the FVM in the regions of the phase diagram where it is possible. We find no qualitative differences for both methods and the quantitative difference is of the same order as presented in this chapter. 

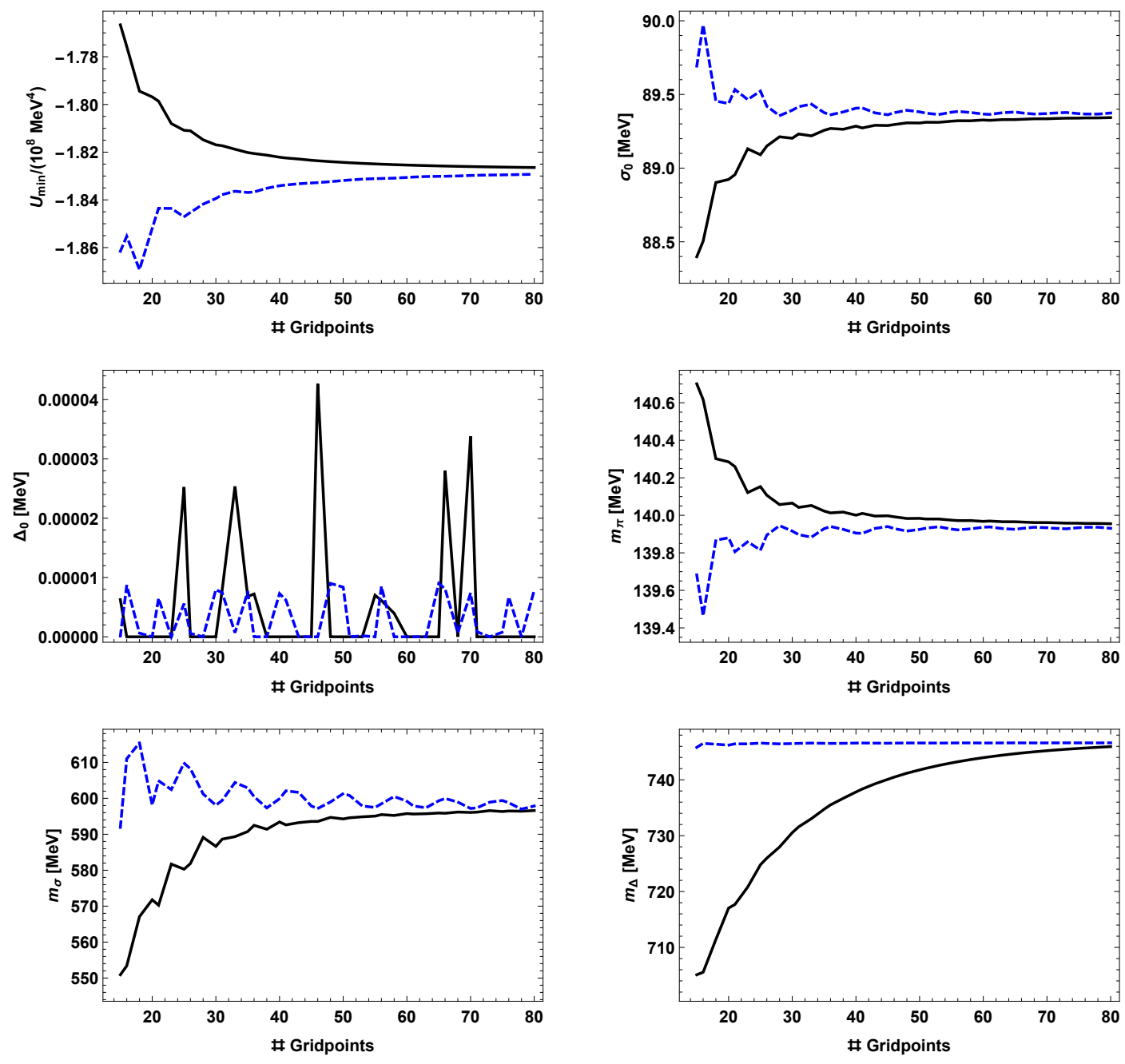

FIGURE 5.8: Black: 2D FDM, blue-dashed: 2D FVM. Various IR observables are plotted with respect to the number of grid points for each dimension. The grid size is fixed to $\sigma_{\max }^{2}=\Delta_{\max }^{2}=180^{2} \mathrm{MeV}^{2}$. Here $T=5 \mathrm{MeV}$ and $\mu=0 \mathrm{MeV}$. The relative errors for each grid configuration are less than $1 \%$. 

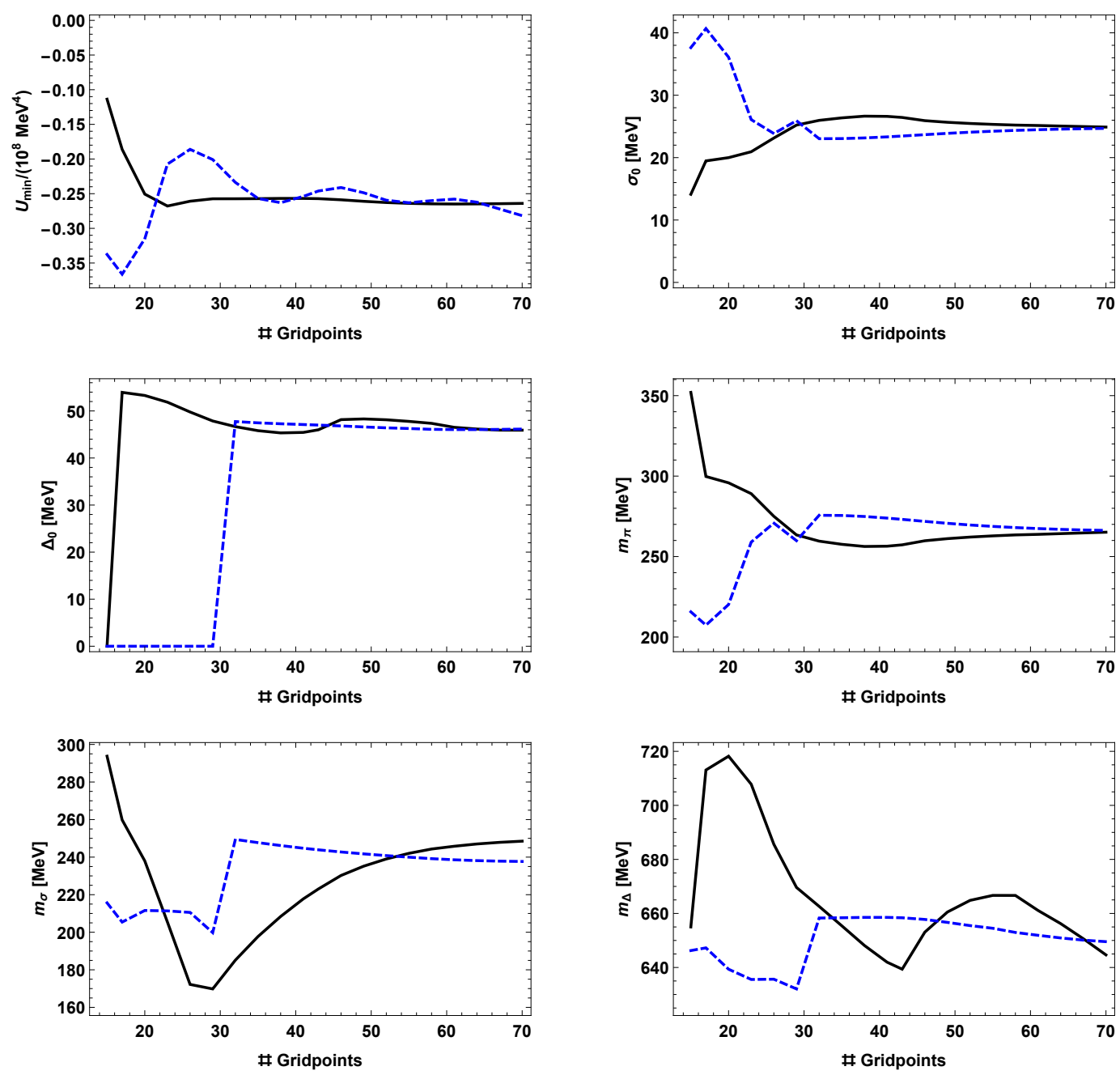

FIGURE 5.9: Black: 2D FDM, blue-dashed: 2D FVM. Plot of the grid-point dependence close to the first-order phase transition line at $T=25 \mathrm{MeV}$ and $\mu=310 \mathrm{MeV}$. Here the results become more uncertain for the $\sigma$ and $\Delta$ masses, since these depend on the second derivatives in the $\sigma$ and $\Delta$ directions, which are extremely sensitive with respect to the position of the minimum. 

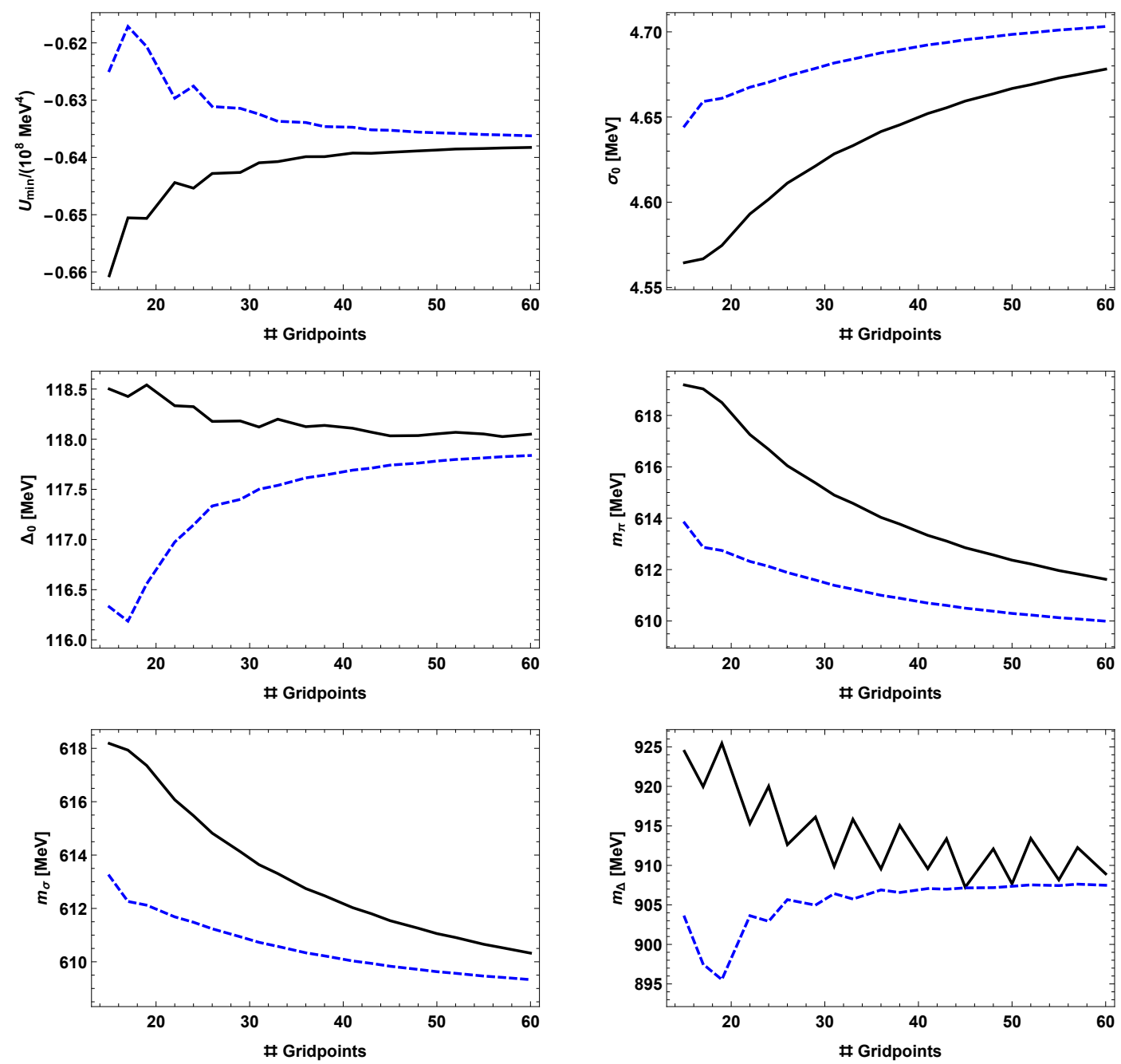

FIGURE 5.10: Black: 2D FDM, blue-dashed: 2D FVM. Plot of the grid-point dependence at $T=35 \mathrm{MeV}$ and $\mu=400 \mathrm{MeV}$. Further away from the first-order phase transition the observables seem to become more reliable again, i.e. the dependence on the grid points becomes small. 

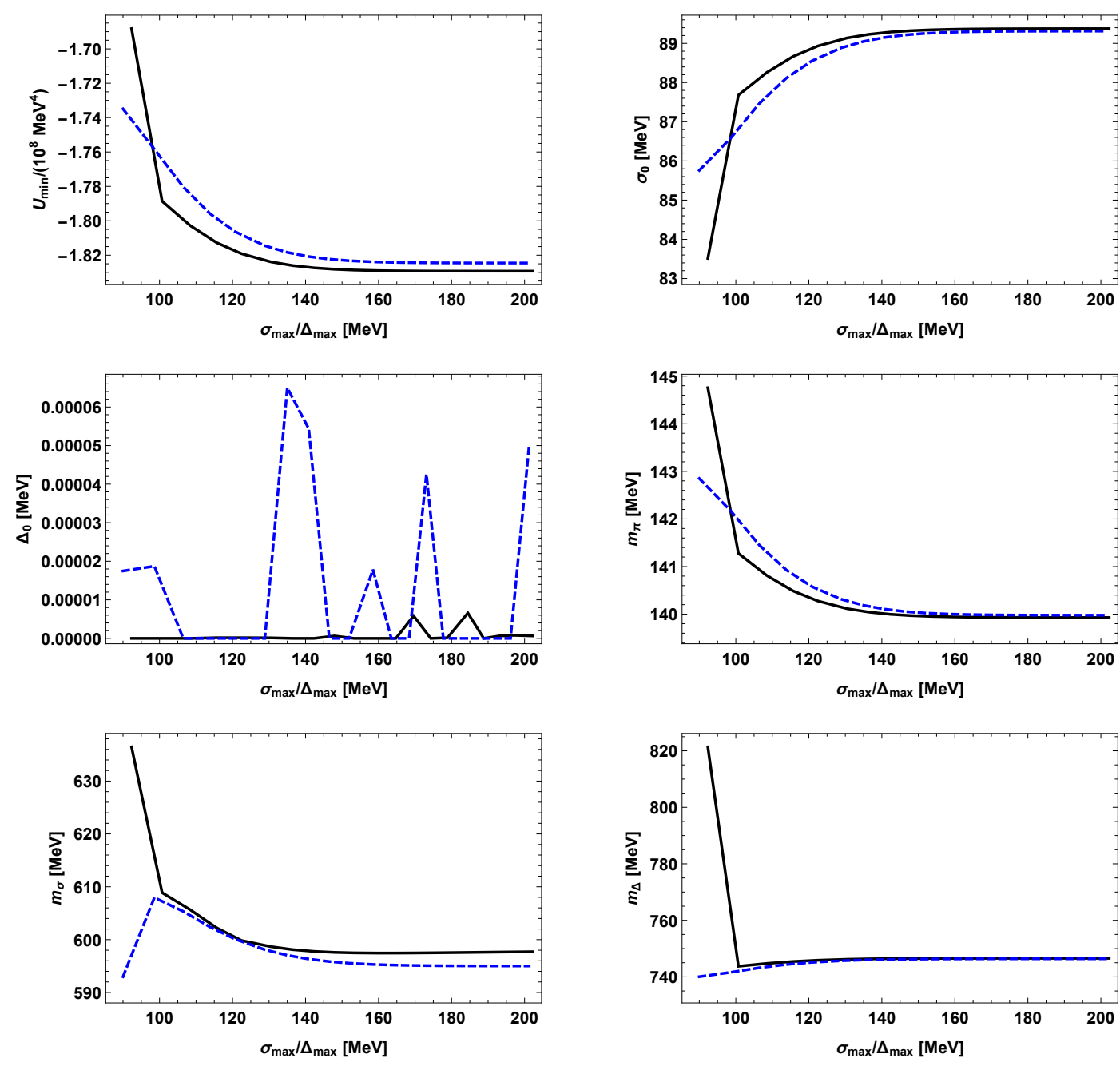

FIGURE 5.11: Black: FDM, blue-dashed: FVM. Various IR observables are plotted in dependence on the grid length. The grid spacing is fixed to $d \Delta^{2}=d \sigma^{2}=180^{2} / 60$ $\mathrm{MeV}^{2}$. Here $T=5 \mathrm{MeV}$ and $\mu=0 \mathrm{MeV}$. The relative errors for each grid configuration are less than $1 \%$. 

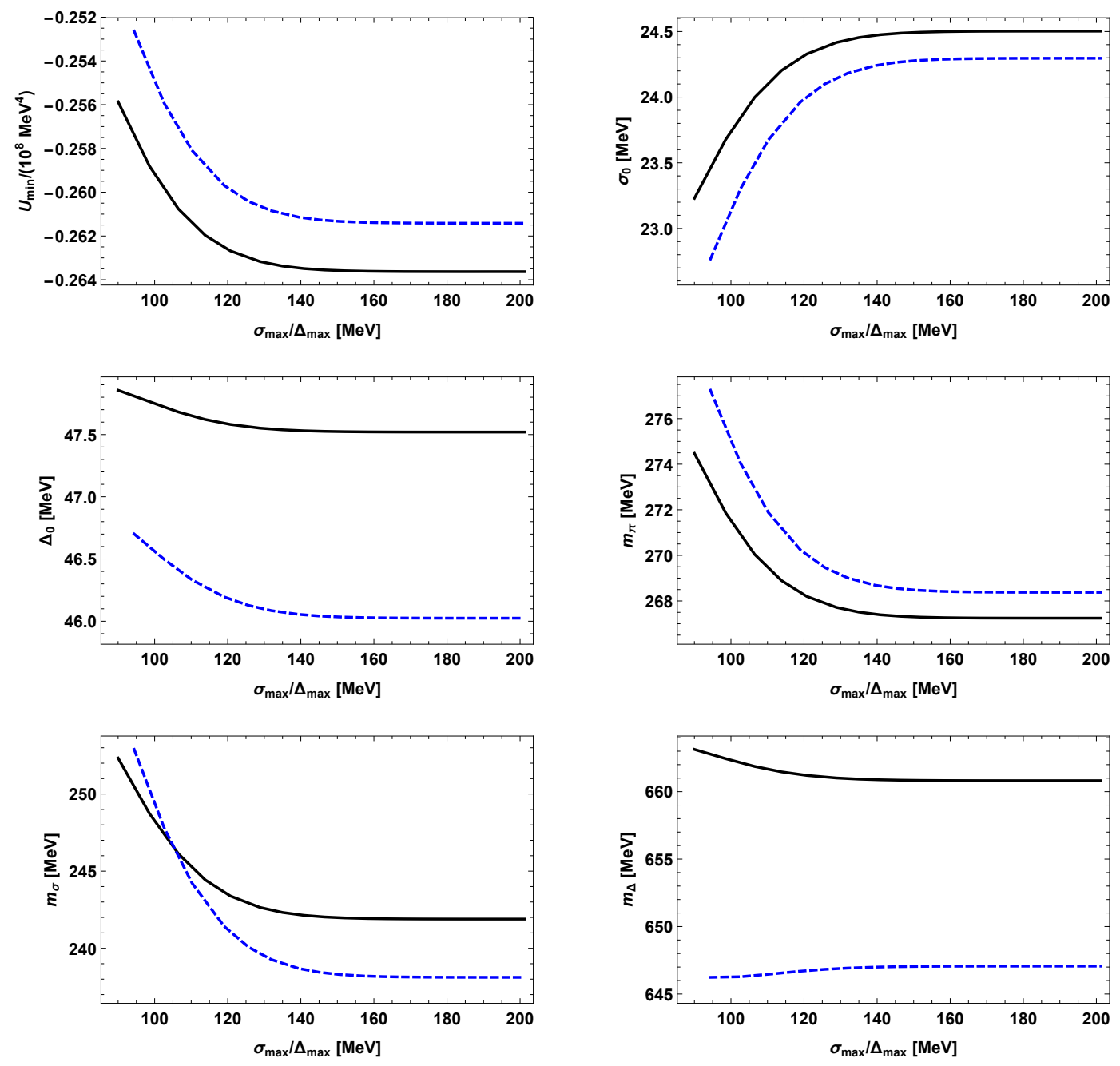

FIGURE 5.12: Black: 2D FDM, blue-dashed: 2D FVM. Plot of the grid-length dependence close to the first-order phase transition line at $T=25 \mathrm{MeV}$ and $\mu=310$ $\mathrm{MeV}$. 

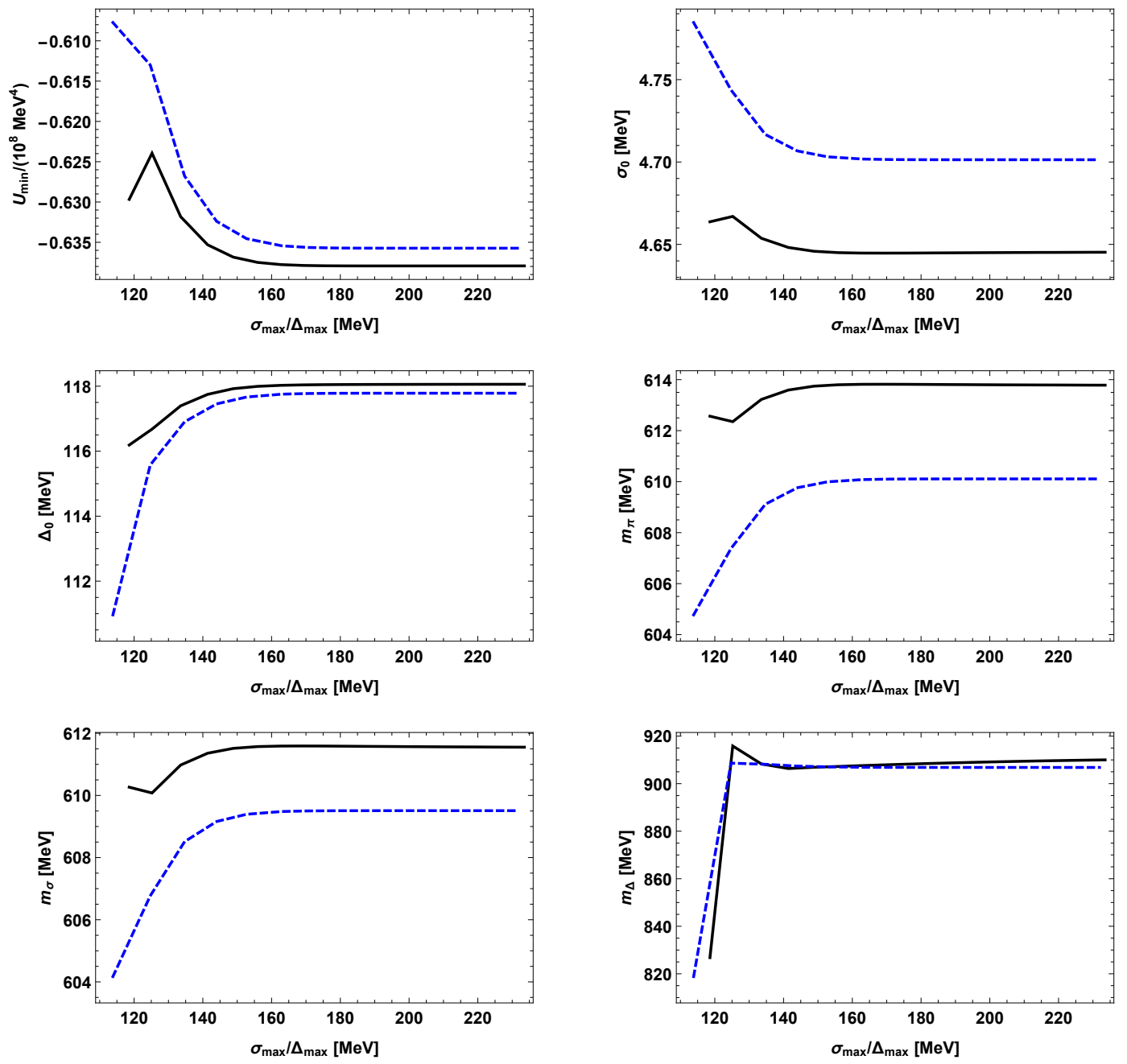

FIGURE 5.13: Black: 2D FDM, blue-dashed: 2D FVM. Plot of the grid-length dependence at $T=35 \mathrm{MeV}$ and $\mu=400 \mathrm{MeV}$. 


\section{Chapter 6}

\section{Results}

In this chapter we present the main results of the FRG part of this thesis. In Section 4.4 we have discussed the flow equation of the quark-meson-diquark model, which we have derived in App. D. Now we will solve this partial differential equation numerically in order to obtain the effective potential in the IR. Subsequently we discuss the results and compare it with the known phase diagram of the quark-meson model in the LPA truncation.

\subsection{The Phase Diagram of the Quark-Meson- Diquark Model}

In the following, the parameters of the UV potential are the same as for the numerical test cases of the last section and listed in Tab. 5.2. These are also the same parameters as in Ref. [1] except for $m_{\Delta}, \lambda_{\Delta}$, and $\lambda_{\sigma \Delta}$, which are new in our model. In this way, we can compare our results with those of Ref. [1]. The diquark coupling $h_{\Delta}$ is not yet fixed as we will vary it in the following.

In Fig. 6.1 we plot the chiral order parameter in the $(T, \mu)$ plane for four different diquark couplings, $h_{\Delta}=0, h_{\Delta}=2, h_{\Delta}=3$ and $h_{\Delta}=4$. In the upper left panel of Fig. 6.1, corresponding to $h_{\Delta}=0$, the phase diagram is almost identical to the QM model phase diagram of Ref. [1]. Even though in the QMD model diquark fluctuations contribute during the flow, for small diquark couplings this does not alter the resulting phase diagram in a significant way when compared to the QM model. We also find the region of negative entropy density as first discovered in Ref. [1]. As expected, no diquark condensate forms with zero diquark coupling.

In the upper right panel we increase the diquark coupling to $h_{\Delta}=2$, which yields a similar phase diagram but now the negative entropy-density region does not extend to arbitrarily large chemical potentials.

In the lower left panel of Fig. 6.1, corresponding to $h_{\Delta}=3$, we notice that the negative entropy density and thus also the backbending almost completely vanishes. Further increasing the diquark coupling moves the phase transition to smaller chemical potentials and completely cures the negative entropy density as seen in the lower right panel of Fig. 6.1. At this point we would like to highlight that the negative entropy densities can also be removed by adding additional meson channels to the model. For instance, in a recent study [105] it was found that the inclusion of vector mesons also 
removes the negative entropy density, provided the vector interaction $G_{\omega}$ is large enough.

In Fig. 6.2 the diquark condensate in the $(T, \mu)$ plane for $h_{\Delta}=2, h_{\Delta}=3$ and $h_{\Delta}=4$ is shown. Interestingly, in the $h_{\Delta}=2$ case the negative entropydensity region ends exactly where the diquark condensate sets in. This seems to support the suspicion of Ref. [1], where the authors named missing diquark degrees of freedom as a possible reason for the occurrence of the negative entropy density region. However, for $h_{\Delta}=3$ a small negative entropydensity region remains, which overlaps with the 2SC phase.

It is also noteworthy to mention that in the vacuum and for $h_{\Delta}=0$ we find the pion decay constant to be $f_{\pi}=92.5 \mathrm{MeV}$ agreeing with Ref. [1]. In the $h_{\Delta}=3$, case however, we find $f_{\pi}=88.9 \mathrm{MeV}$. This shows that even in vacuum the diquark loops contributing to the flow equation affect the resulting effective potential in the IR, albeit only slightly.

\section{Chiral Condensate $\sigma[\mathrm{MeV}]$}
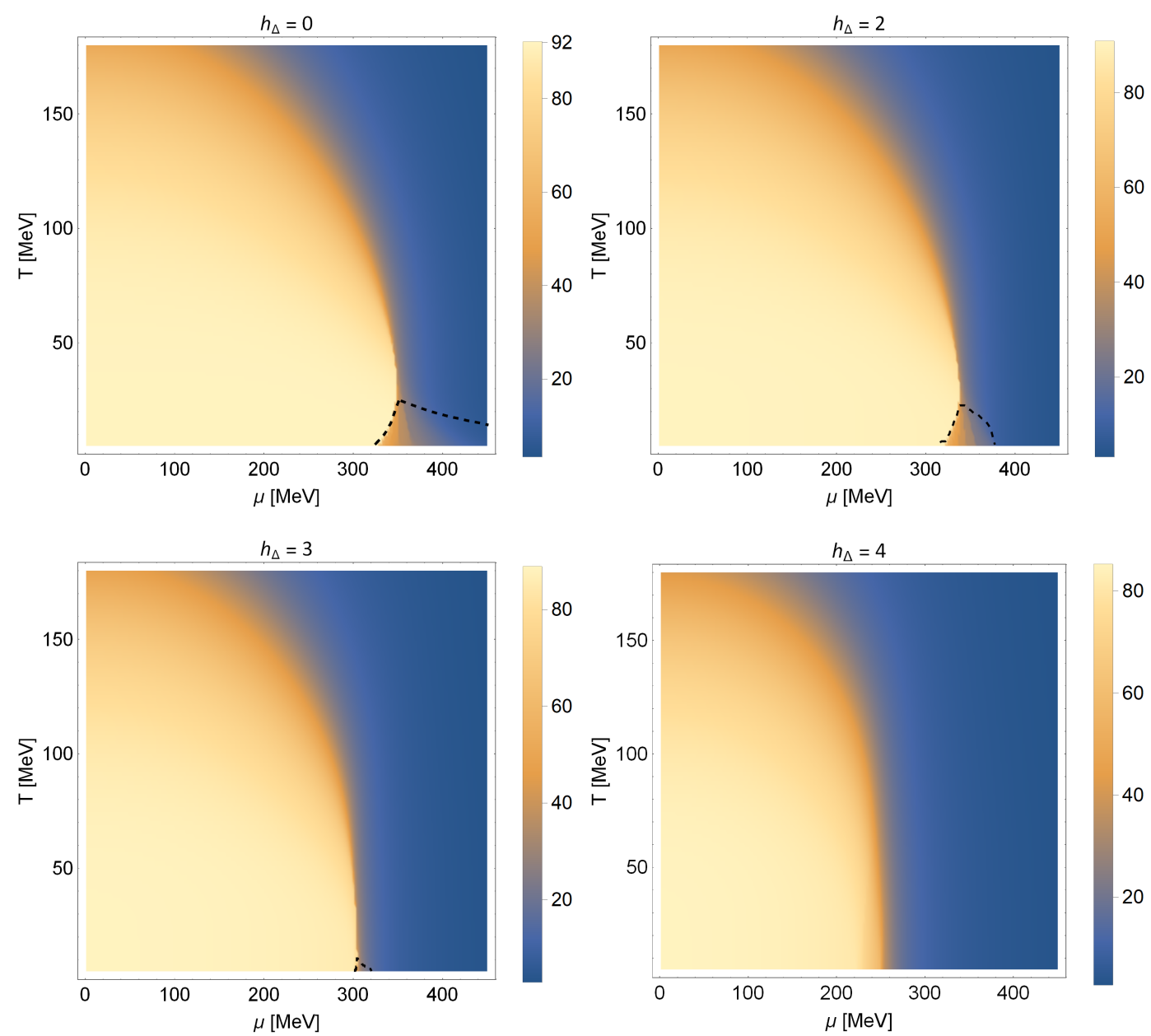

FIGURE 6.1: The chiral condensate in the $(T, \mu)$ plane for the diquark couplings $h_{\Delta}=0, h_{\Delta}=2, h_{\Delta}=3$ and $h_{\Delta}=4$. Below the black-dashed line the entropy density is negative. For $h_{\Delta}=4$ the negative entropy density region completely vanishes. 


\section{Diquark Condensate $\Delta[\mathrm{MeV}]$}
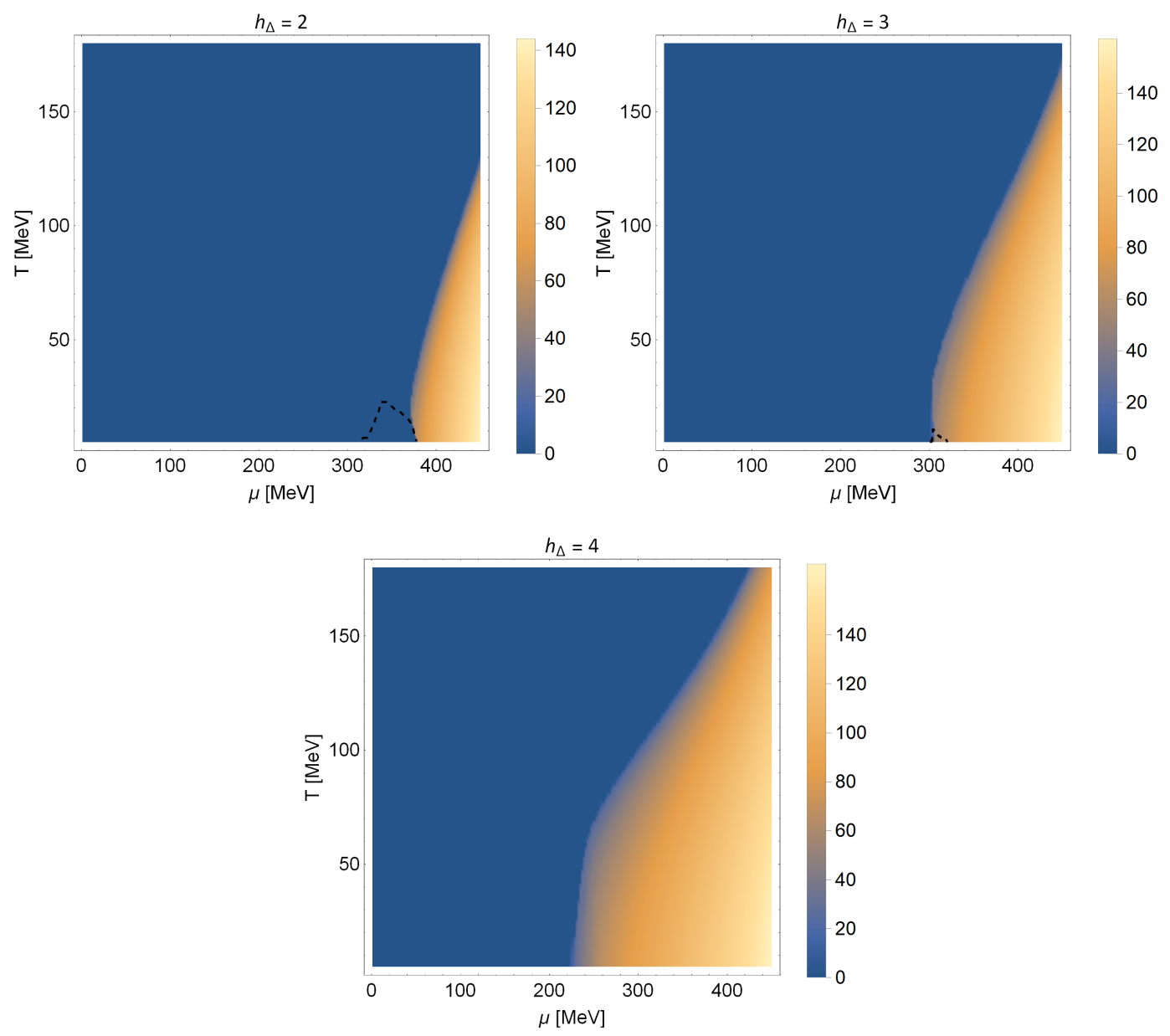

FIGURE 6.2: The diquark condensate in the $(T, \mu)$ plane for the diquark couplings $h_{\Delta}=2, h_{\Delta}=3$ and $h_{\Delta}=4$. Below the black-dashed line the entropy density is negative.

In Figs. 6.3a-6.3c we present the scale dependence of the pion decay constant, the diquark condensate, and the curvature meson masses (definitions are given in Eqs. (D.21)-(D.24)) for three interesting points of the phase diagram for the diquark coupling $h_{\Delta}=3$. One in the vacuum, one close to the first-order chiral phase transition and one in the 2SC phase. The plot for the vacuum result is the most recognizable one as it is very similar to the flows typically obtained in vacuum quark-meson model studies [70,106]. The diquark mass drops slightly as we integrate out the degrees of freedom but it remains comparatively large and therefore its contribution to the effective potential is small.

Particularly interesting is Fig. 6.3b where the complex interplay between the chiral and diquark degrees of freedom unfolds. First the chiral condensate continuously increases as the fluctuations are successively integrated out and at around $k=450 \mathrm{MeV}$ the diquark condensate sets in as well. However then there is jump at around $k=200 \mathrm{MeV}$ affecting both the chiral and diquark condensate. 
In Fig. 6.3c we notice the almost complete restoration of chiral symmetry with a negligible chiral condensate and degenerate chiral partners.

In Fig. $6.3 \mathrm{~d}$ we display the full $\mu$-dependence at $T=5 \mathrm{MeV}$. Here one can nicely see that the diquark condensate becomes non-vanishing exactly at the point where the diquark mass becomes approximately zero, exhibiting a typical behavior for a radial mode at the phase transition.
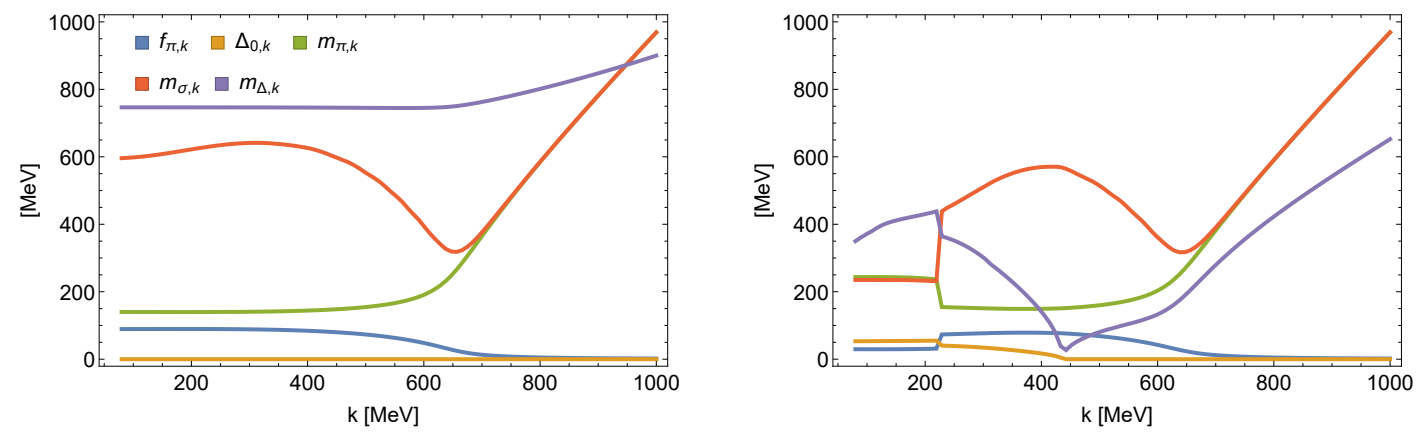

(A) scale dependence at

$(T, \mu)=(5 \mathrm{MeV}, 0 \mathrm{MeV})$

(B) scale dependence at

$(T, \mu)=(5 \mathrm{MeV}, 310 \mathrm{MeV})$
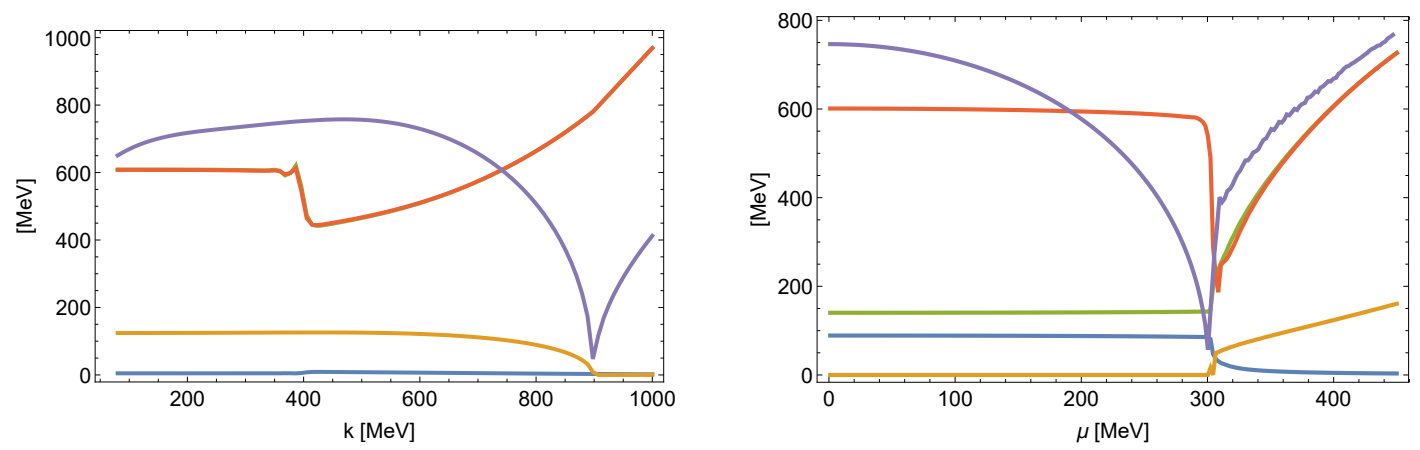

(C) scale dependence at

(D) $\mu$ dependence of IR observables at $(T, \mu)=(5 \mathrm{MeV}, 400 \mathrm{MeV})$

$T=5 \mathrm{MeV}$

FIGURE 6.3: Scale-dependent curvature masses and the pion decay constant as well as the diquark gap for $h_{\Delta}=3$. In the lower right panel the dependence on the chemical potential is displayed where the quantities are evaluated in the IR. 


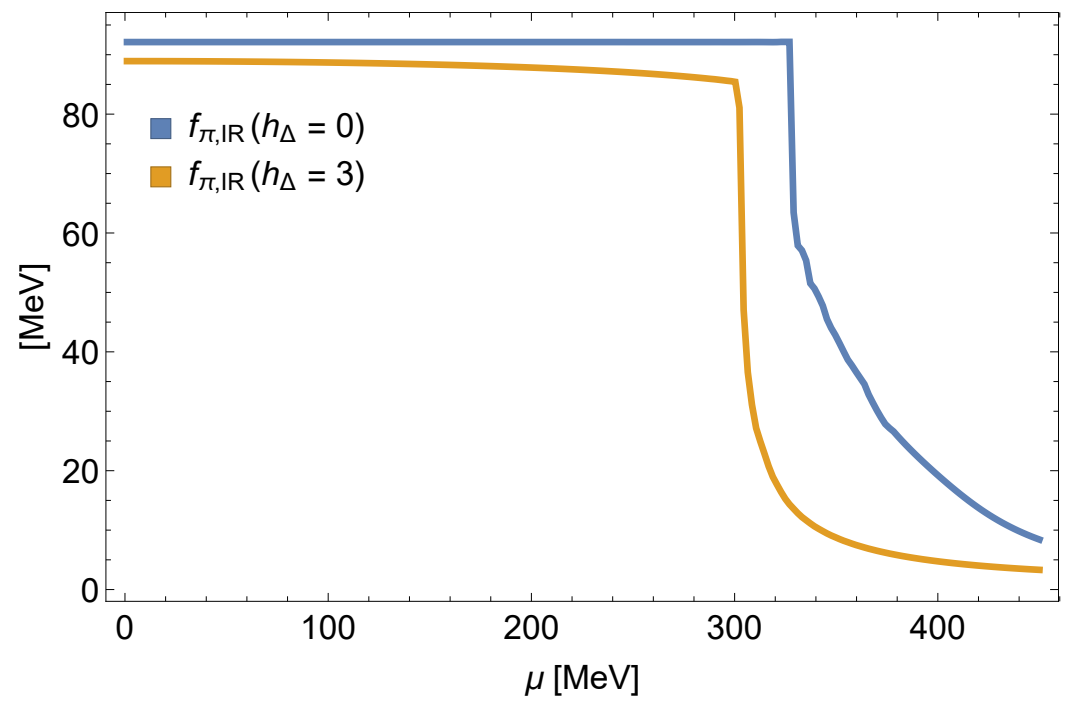

FIGURE 6.4: $\mu$-dependence of $f_{\pi}$ at $T=5 \mathrm{MeV}$ for both $h_{\Delta}=0$ and $h_{\Delta}=3$. The latter violates the Silver-Blaze property sligthly.

Another interesting aspect is the violation of the Silver-Blaze property for $h_{\Delta}=3$. The Silver-Blaze property $[107,108]$ states that the partition function at zero temperature is independent of the chemical potential for $\mu<\mu_{\text {crit }}$, where $\mu_{\text {crit }}$ denotes some critical value for the chemical potential. While the violation of this property is barely visible in Fig. 6.3d, in Fig. 6.4 we compare the $\mu$-dependence of the pion decay constant for both $h_{\Delta}=0$ and $h_{\Delta}=3$ in more detail. For finite diquark couplings the chiral condensate shrinks very slightly but continuously even before the phase transition. The violation of the Silver-Blaze property can be seen even more clearly by plotting the quark number density. For this purpose, we provide an additional plot in Fig. 6.5. 


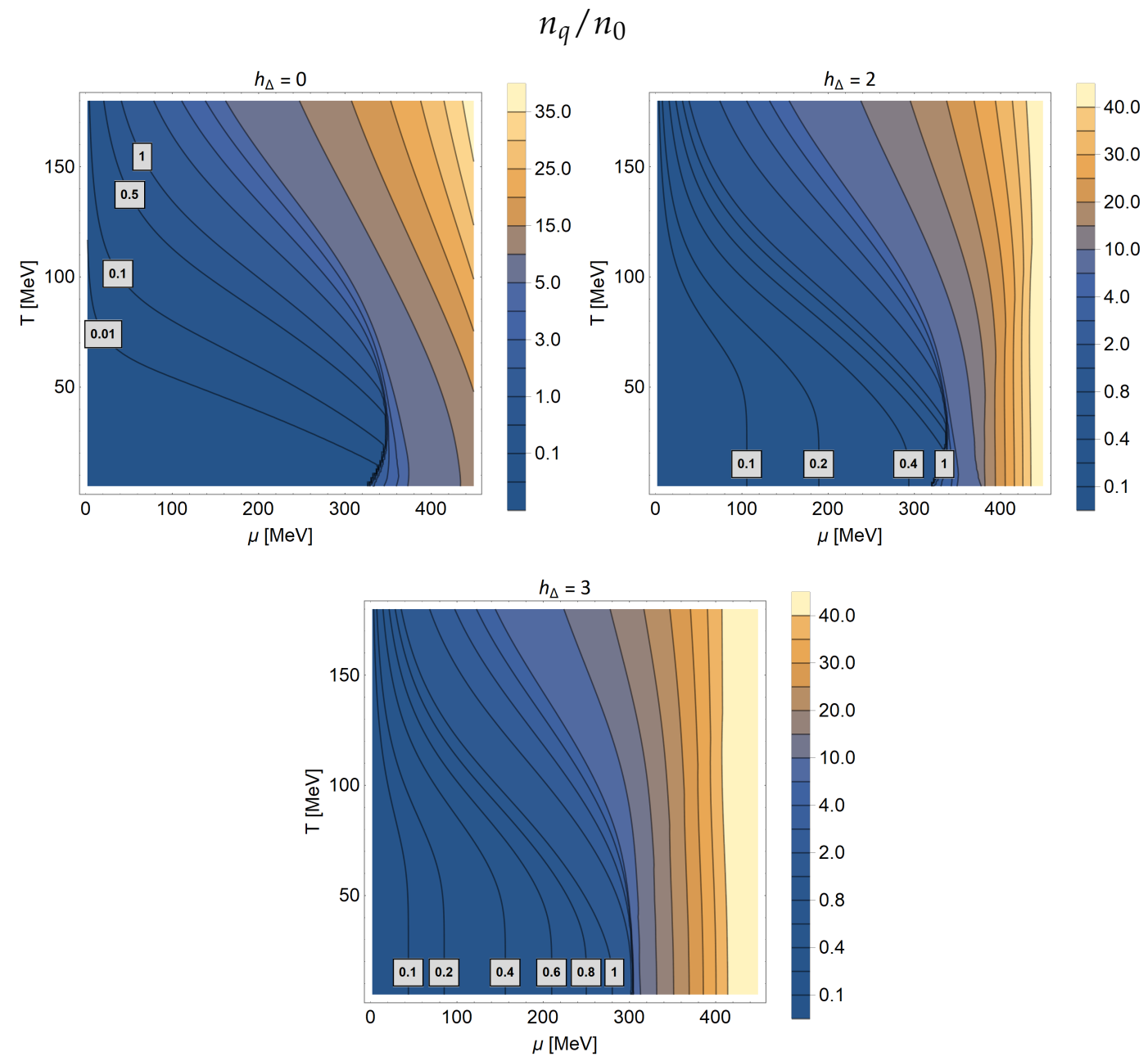

FIGURE 6.5: The quark number density compared to the nuclear saturation density, $n_{q} / n_{0}$, is plotted in the $(T, \mu)$ plane for the diquark couplings $h_{\Delta}=0, h_{\Delta}=2$, and $h_{\Delta}=3$. For non-zero diquark coupling the quark number density starts increasing from $\mu=0$ violating the Silver-Blaze property.

The observation of the violation of the Silver-Blaze property is in agreement with Refs. $[65,69]$ where the author also found a violation of the SilverBlaze property for the quark-meson-diquark model of two-and three-color QCD and analyzed it in the FRG context in much detail. The author came to the conclusion that if the Silver-Blaze property holds true at the initial scale, it will be preserved by the flow equation and therefore should also be fulfilled in the IR. But this is only true, if the full momentum dependence of all quantities is taken into account, which is not the case for the local potential approximation. The other way around, the slight violation of the Silver-Blaze property might imply that there is only a mild momentum dependence that is not included in our truncation, affecting relevant observables only slightly. 


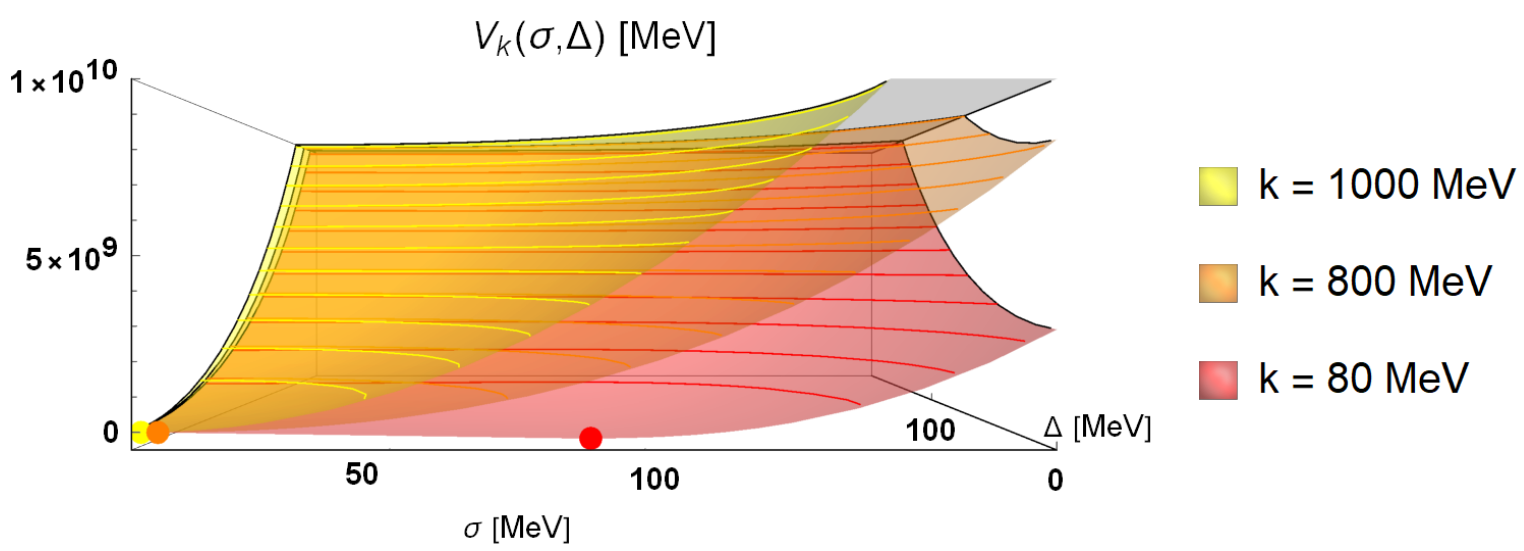

FIGURE 6.6: Flow of the two-dimensional effective potential of the quark-mesondiquark model in the vacuum $(T, \mu)=(5 \mathrm{MeV}, 0)$. The dots denote the position of the minima. The evolution of the potential in the $\Delta$ direction is minimal and barely affects the location of the minima, which are almost identical to the QM model ones.

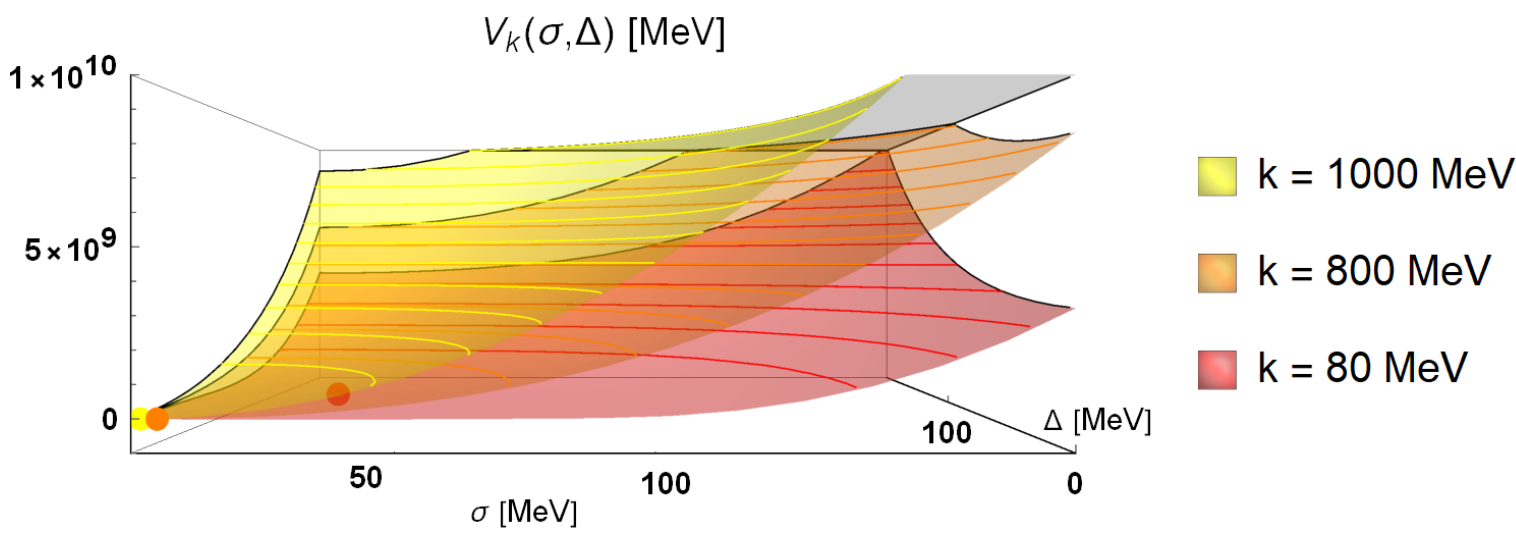

FIGURE 6.7: Flow of the two-dimensional effective potential of the quark-mesondiquark model close to the phase transition $(T, \mu)=(5 \mathrm{MeV}, 310 \mathrm{MeV})$.

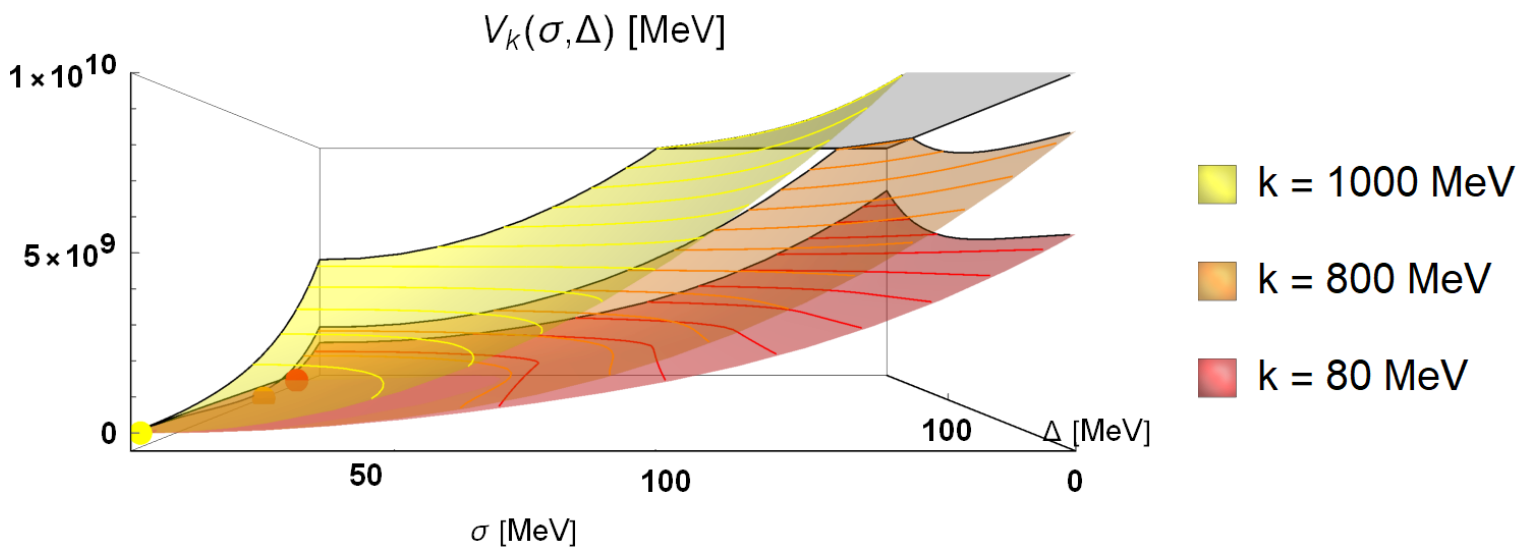

FIGURE 6.8: Flow of the two-dimensional effective potential of the quark-mesondiquark model for large chemical potential, $(T, \mu)=(5 \mathrm{MeV}, 400 \mathrm{MeV})$. 
In Figs. 6.6-6.8 we provide additional 3D plots for the flow of the full $2 D$ potential for $h_{\Delta}=3$ to get an idea how the full 2D potential evolves during the flow.

\subsection{Stability Analysis}

Before presenting the results for the stability analysis of the quark-mesondiquark model, we would like to reproduce the stability analysis of Ref. [1]. But here we will use the finite-volume method to cross-check the results of Ref. [1], where a finite-difference method was used. Our result using the finite-volume method is presented in Fig. 6.9. Two methods to determine the instability of the two-point function are employed. In the first approach, we evaluate the static pion two-point function at the IR value of the chiral condensate, i.e., $\sigma=\sigma_{0, I R} \equiv f_{\pi}$. We then start integrating from $k_{\Lambda}$ to $k_{0}$, where $k_{0}$ denotes the scale where the two-point function develops a zero crossing for the first time.

The second approach is to evaluate the minimum at an intermediate scale $k_{0}>k_{\mathrm{IR}}$, i.e., $\sigma=\sigma_{0, k_{0}}$. Subsequently, one then evaluates the two-point function at this minimum and solves the flow equation for the two-point function until the intermediate scale $k_{0}$ is reached. This is repeated for various $k_{0}$ towards the IR, whereas $k_{0}=k_{\mathrm{IR}}$ corresponds to the first method. This approach is similar to a Taylor expansion with a scale-dependent expansion point, which is often used in FRG studies [67, 109].

It would be preferable to render the chiral order parameter fully scaledependent, however this is not part of the truncation, this is why the above two methods were introduced.

Our results are in excellent agreement with the results of Ref. [1]. This gives us confidence that the finite-difference method used in previous studies did not lead to systematic numerical errors. 

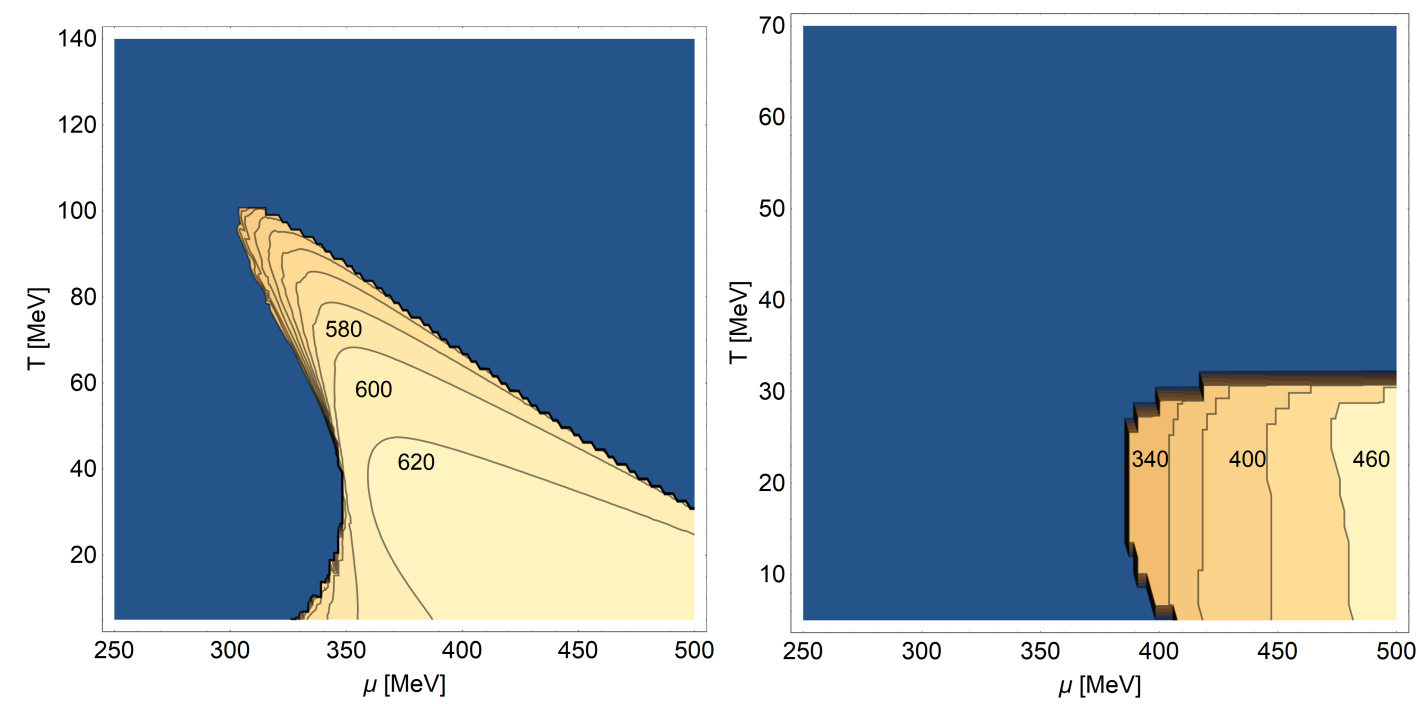

FIGURE 6.9: Region in the $(T, \mu)$-plane where the pion two-point function develops a zero crossing. In the left panel the numbers denote the flow scale $k_{0}$ where the two-point function evaluated at $\sigma_{0, I R}$ develops a zero crossing for the first time. In the right panel the two-point function is evaluated at the scale-dependent minima $\sigma_{0, k_{0}}$ instead.

In Figs. 6.10 and 6.11 we present the region of instability for the quarkmeson-diquark model with diquark coupling $h_{\Delta}=2$ and $h_{\Delta}=3$, respectively ${ }^{1}$. We immediately notice that for both cases the region of instability compared to the QM model in Fig. 6.9 is significantly smaller.

\footnotetext{
${ }^{1}$ The case $h_{\Delta}=0$ is almost identical to Fig. 6.9.
} 


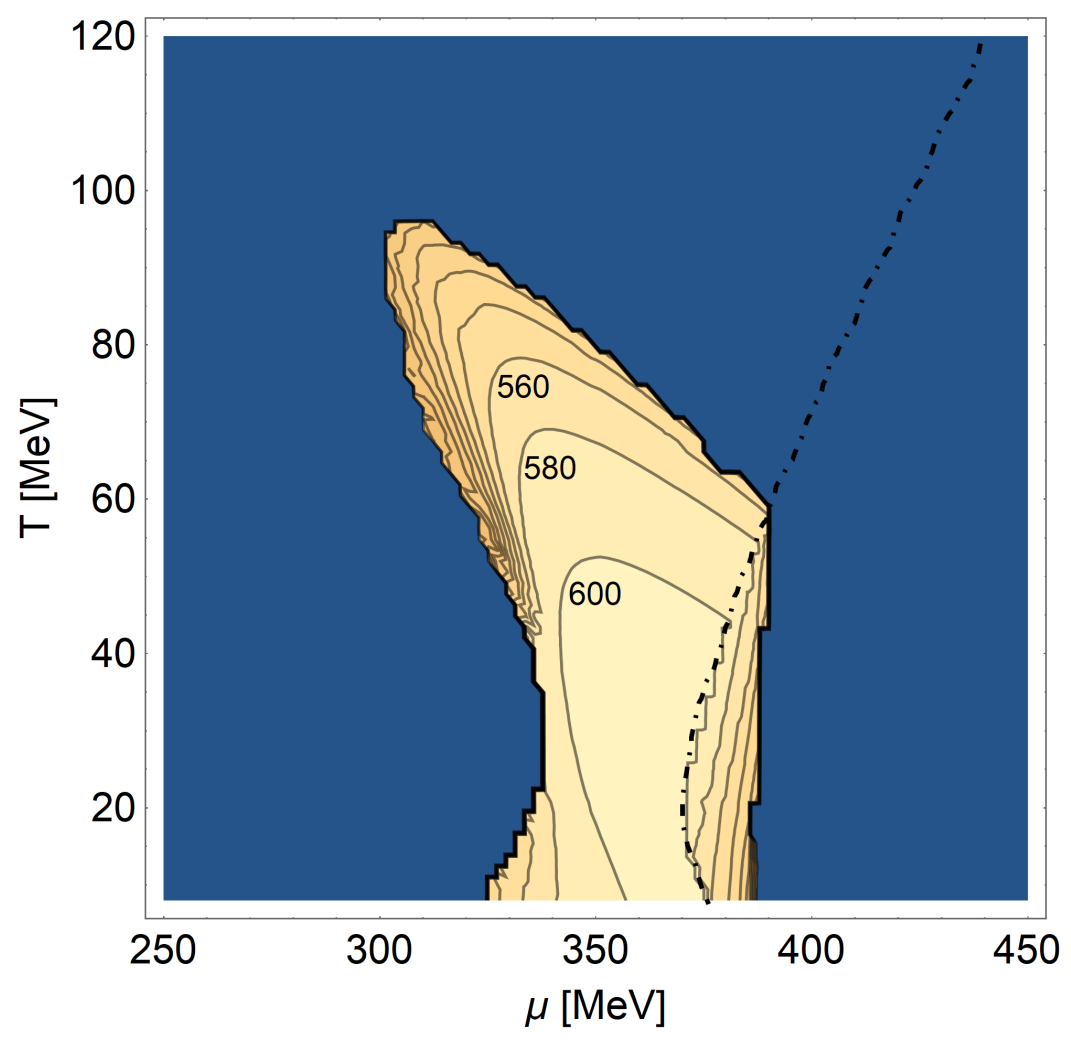

FIGURE 6.10: Region of instability for $h_{\Delta}=2$ evaluated at $\sigma_{0, I R}$. The black dashdotted line denotes the onset of the diquark condensate. If we consider the scaledependent minima $\sigma_{0, k_{0}}$ we find no instabilities. 


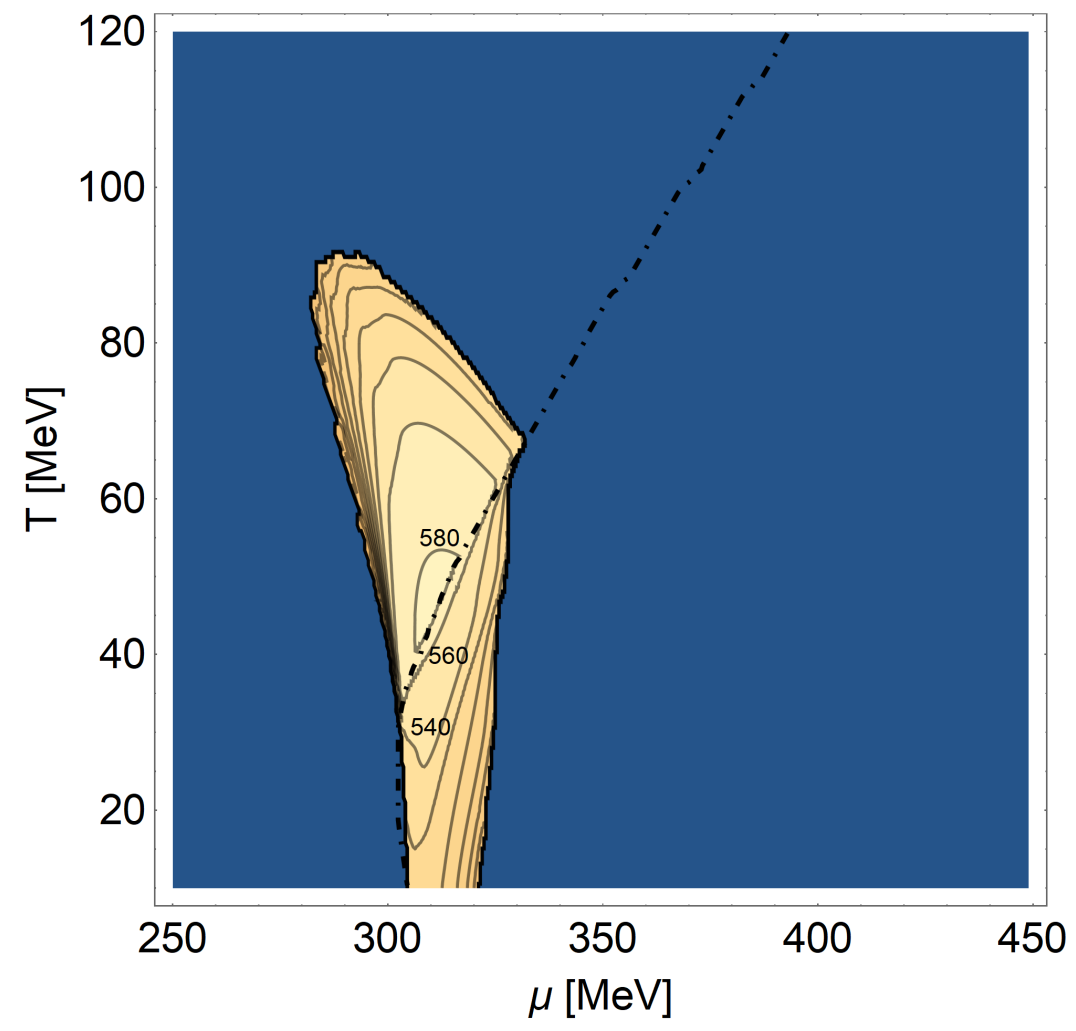

FIGURE 6.11: Region of instability for $h_{\Delta}=3$ evaluated at $\sigma_{0, I R}$. The black dashdotted line denotes the onset of the diquark condensate. If we consider the scaledependent minima $\sigma_{0, k_{0}}$ we find no instabilities.
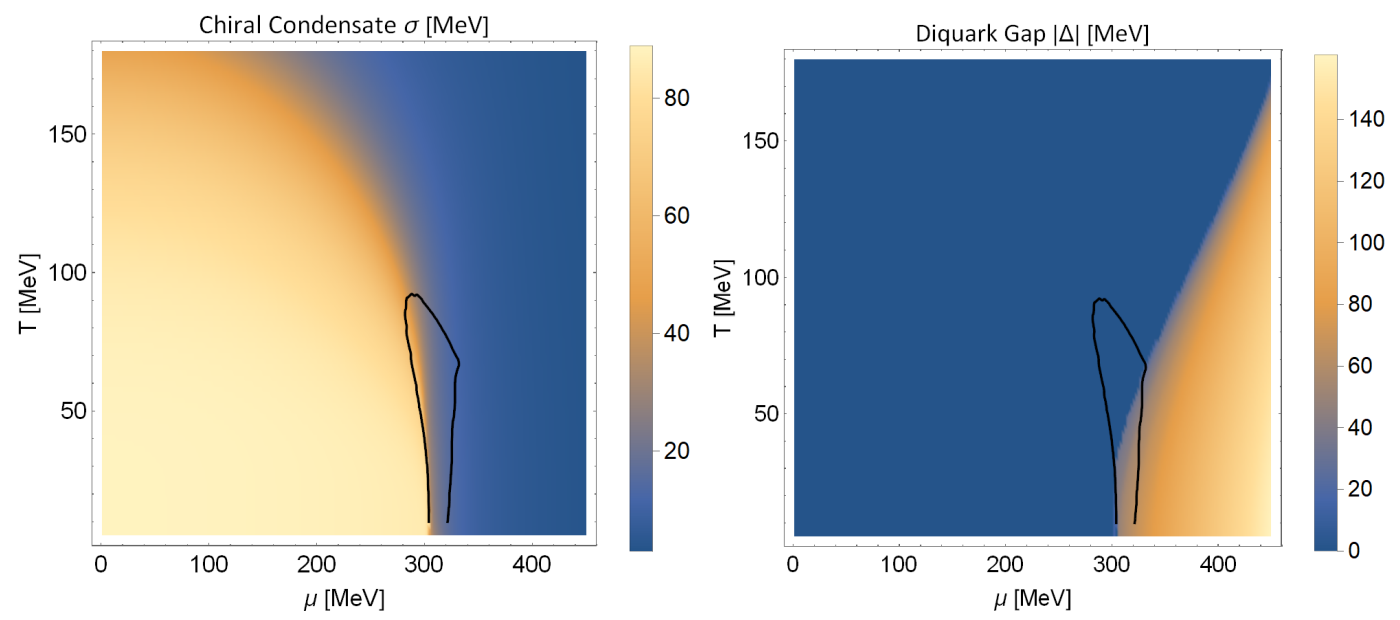

FIGURE 6.12: The phase diagram for $h_{\Delta}=3$. The black line denotes the region of instability for $\Gamma_{k, \pi}^{(2)}$ evaluated at $\sigma_{\mathrm{IR}}$. If we consider the scale-dependent minima $\sigma_{k_{0}}$ we find no instabilities.

In the upper half of each of the instability regions, we notice that the scale $k_{0}$ at which a zero-crossing develops first increases until it touches the diquark-onset boundary line. From there towards larger $\mu$ and smaller $T$ the scale $k_{0}$ then decreases again. Therefore the effect of the diquark condensate is very significant as it suppresses the $k_{0}$, i.e., the zero-crossing develops later 
in the evolution of the flow equation compared to the QM model. Then, the instability region vanishes as the chemical potential and thus also the diquark condensate further increases. This behavior seems not to be a coincidence and therefore it might be natural to interpret that there exists a possible competition between the 2SC phase and a hypothetical inhomogeneous phase. We have already discussed the problem on how to interpret the instabilities occuring during the flow in Chapter 4.5. Here we would like to reiterate that from the discovery of an instability region it does not immediately follow that in the same region an inhomogeneous phase exists. However, this can be seen as a hint, which is supported by two facts:

1. The region of instability is found in the vicinity of the chiral phase transition, where in QM and NJL model mean-field studies inhomogeneous phases are typically found.

2. There is a competition between the instability region and the $2 \mathrm{SC}$ phase, resembling the competition of the chiral inhomogeneous and 2SC phase in NJL mean-field studies $[21,25,75]$.

On the other hand, we find no instabilities using the second method and thus we cannot come to an unambiguous conclusion. An unequivocal result could be only obtained in a setting where the order parameters are made inhomogeneous, i.e., $\sigma=\sigma(\vec{x})$, for instance through a chiral density-wave Ansatz, because then the evolution of the effective potential changes at $k_{0}$, where the system wants to form an inhomogeneous state, most likely leading to a different IR potential and IR two-point function.

\subsection{Renormalization-Group Consistent UV Poten- tials}

All the previously discussed results in this chapter have been obtained by using the UV-initial potential Ansatz in Eq. (5.25). The problem with our Ansatz for the UV-initial potential is that it has no explicit dependence on temperature and chemical potential, it is the same for the entire phase diagram. However, we expect a dependence as our cutoff scale cannot be considered large enough to be independent of these external parameters. A novel way to introduce a temperature- and chemical potential-dependent UV potential has been proposed in Ref. [110]. In this work the authors used the concept of RG consistency in order to retrospectively introduce a dependence on external parameters to the UV potential.

RG consistency states that the full quantum effective action does not depend on the cutoff parameter, i.e.,

$$
\Lambda \frac{d \Gamma}{d \Lambda}=0
$$


This is evidently in contrast to our Ansatz in Eq. (5.25), because there the UV potential has no dependence on $\Lambda$ at all and therefore changing the cutoff will necessarily lead to a different flow yielding a different effective action in the IR.

However, one can render the effective action RGconsistent in a trivial way, namely by introducing a new $\Lambda$-dependent UV potential. This works as follows: We define our initial Ansatz in Eq. (5.25) at an intermediate cutoff scale by relabeling $\Lambda \rightarrow \Lambda^{\prime}$. That is, $U_{\Lambda, \mathrm{QMD}} \rightarrow U_{\Lambda^{\prime}, \mathrm{QMD}} \cdot \Lambda^{\prime}$ is now a label for an intermediate cutoff, whereas $\Lambda>\Lambda^{\prime}$ labels the proper cutoff for which RG consistency is fulfilled. Then we integrate the flow equation backwards in order to obtain $\Gamma_{\Lambda}$. In this way, the RG-consistency requirement is trivially fulfilled without the need to modify our Ansatz in Eq. (5.25).

The dependence on external parameters such as temperature and chemical potential can then be introduced in the following way: We integrate the $U_{\Lambda^{\prime}, \mathrm{QMD}}$ up to $U_{\Lambda, \mathrm{QMD}}$ using Eq. (4.32) for $T=\mu=0$. Then from $U_{\Lambda, \mathrm{QMD}}$ we solve the flow equation in the usual way for a specific $(T, \mu)$ pair in the phase diagram. This yields a dependence of $U_{\Lambda^{\prime}, \mathrm{QMD}}$ on the temperature and chemical potential. In order to fulfill the requirement of RG consistency, one needs to perform this approach for increasing $\Lambda$ until the full quantum effective action fulfills Eq. (6.1).

Unfortunately, there is a caveat when applying this approach to the full flow equation with bosonic fluctuations. As we have discussed earlier, the flow equation for the effective potential is actually a convectiondiffusion equation and therefore solving the flow equation backwards in flow time is ill-posed. Fortunately, there is a way to circumvent this problem by setting the bosonic contribution to zero when integrating from $\Lambda^{\prime}$ to $\Lambda$. This amounts to a mean-field approximation

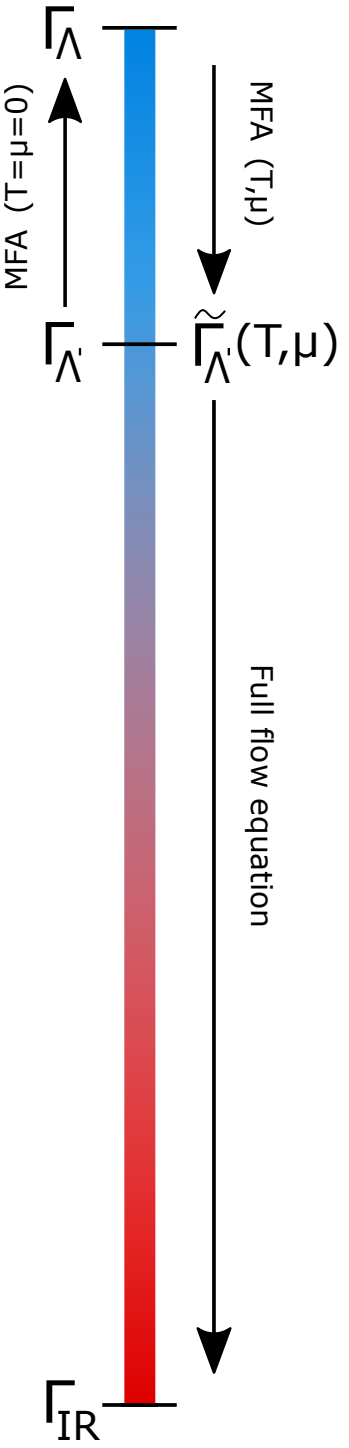

FIGURE 6.13 for which no conceptional issues arise when integrating backwards in flow time.

The strategy is conceptionally visualized in Fig. 6.13. We start with $U_{\Lambda^{\prime}}=$ $\Gamma_{\Lambda^{\prime}} / V$ using Eq. (5.25) as our initial effective potential. Then we remove the bosonic contributions in Eq. (4.32) yielding

$$
\begin{aligned}
\partial_{k} U_{\Lambda^{\prime}, \mathrm{MFA}} & =-\frac{k^{4}}{3 \pi^{2}}\left[2 \sum_{ \pm} \frac{1 \pm \frac{\mu}{E_{k}}}{E_{k}^{ \pm}} \tanh \frac{E_{k}^{ \pm}}{2 T}+\frac{1}{E_{k}}\left(\tanh \frac{E_{k}-\mu}{2 T}+\tanh \frac{E_{k}+\mu}{2 T}\right)\right] \\
& \equiv S_{\Delta}(\phi, \Delta)+S_{\phi}(\phi) .
\end{aligned}
$$


Subsequently, we use Eq. (6.2) in order to obtain $U_{\Lambda, \mathrm{QMD}}$ :

$$
\begin{array}{r}
U_{\Lambda, \mathrm{QMD}}=U_{\Lambda^{\prime}, \mathrm{QMD}}+\int_{k=\Lambda^{\prime}}^{\Lambda} \mathrm{d} k\left[\left.S_{\Delta}(\phi, \Delta)\right|_{T=\mu=0}+\left.S_{\phi}(\phi)\right|_{T=\mu=0}\right. \\
\left.+\frac{\mu^{2}}{2}\left(\left.\partial_{\mu}^{2} S_{\Delta}(\phi, \Delta)\right|_{T=\mu=0}\right)\right] .
\end{array}
$$

Note, that the term $\sim \mu^{2}$ is needed for the correct renormalization of the chemical potential of the diquarks. If we were to omit this term then the Silver-Blaze property would be violated [108]. The $T$ - and $\mu$-dependent UV potential is then obtained by integrating back to $\Lambda^{\prime}$ :

$$
U_{\Lambda^{\prime}, \mathrm{QMD}}(T, \mu)=U_{\Lambda, \mathrm{QMD}}+\int_{k=\Lambda}^{\Lambda^{\prime}} \mathrm{d} k\left[S_{\Delta}(\phi, \Delta)+S_{\phi}(\phi)\right] .
$$

Now with the $T$ - and $\mu$-dependent UV potential in hand we use the full flow equation (4.32) to integrate down to the IR.
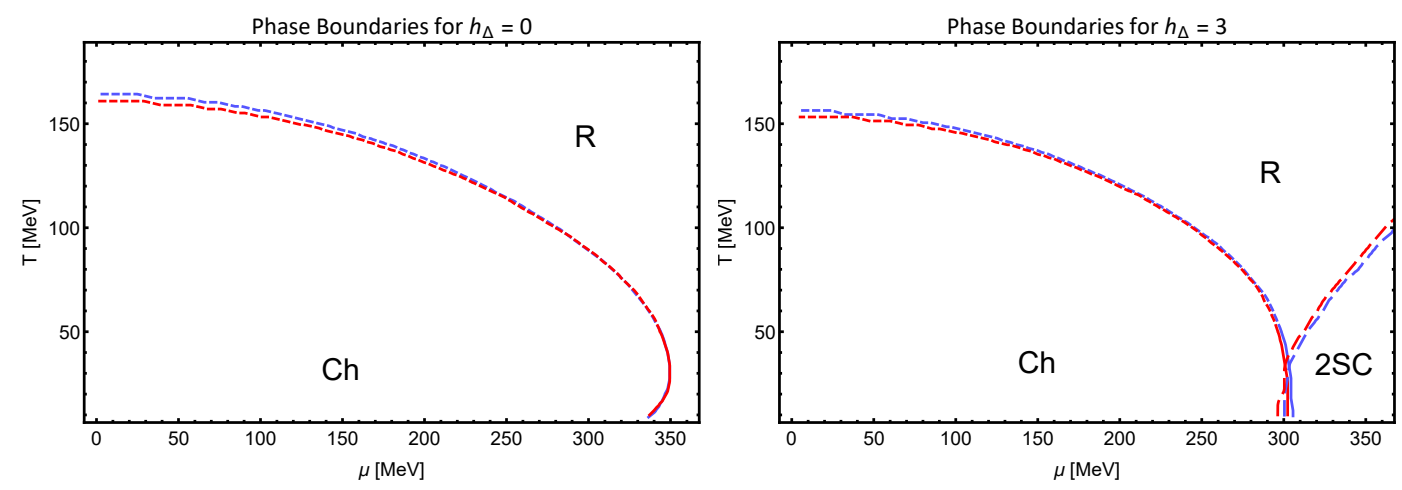

FigURE 6.14: Blue: $\Lambda=\Lambda^{\prime}$. Red: $\Lambda=5 \Lambda^{\prime}$. The dashed line in the $\chi S B$ phase is the contour line at $\sigma / \sigma_{0}=0.75$. The solid line denotes the first-order chiral phase transition line. The larger dashed lines denote the diquark onset for the $2 \mathrm{SC}$ phase.

Using the RG-consistency procedure we compare the phase boundaries in Fig. 6.14 for $h_{\Delta}=0$ and $h_{\Delta}=3$. Qualitatively we find similar corrections to the phase boundaries as in Ref. [110]. There the authors have found that the RG-consistent MFA approach shrinks the chiral symmetry-broken phase while the 2SC phase is enlarged. Furthermore, the first-order phase transition sets in for slightly smaller chemical potential.

In our case the corrections are smaller compared to Ref. [110]. This is most likely due to the fact that we used the modified RG-consistent approach explained before. The UV potential only contains corrections at the meanfield level but the full flow also contains corrections from the bosonic loops and thus rendering the purely fermionic corrections relatively smaller.

We find that the influence of the RG-consistent corrections on all other results presented in this chapter are of the same order of magnitude. 


\section{Chapter 7}

\section{Conclusions and Outlook}

This thesis can be seen as a small part of a larger research project, namely the Collaborative Research Center TR-211, whose goal is to better understand strongly interacting matter under extreme temperature and density by using state-of-the-art lattice methods as well as non-perturbative methods such as the functional renormalization group. Of particular interest in this research project is the study of the chiral phase transition, since its precise location and properties are of great importance for our understanding of the early Universe and compact stars.

This thesis contributed to this research program by studying the competition between inhomogeneous chiral condensation with homogeneous twoflavor color superconductivity.

In the first part of this thesis we have looked for this competition in the phase diagram of the chirally symmetric two-flavor NJL model using the mean-field approximation. We successfully derived the grand potential for a generic inhomogeneous condensate, expressed as an energy integral over a generic density of states. This enabled us to employ already known densities of states for inhomogeneous chiral condensates and, if in the future more densities of states for inhomogeneous modulations of the chiral order parameter in the absence of two-flavor color superconductivity are found, it will be straightforward to include them in our framework, namely by inserting the density of states into Eq. (3.23). Here we have employed the CDW and the RKC densities of states. We have found that for some diquark couplings the inhomogeneous chiral and the homogeneous 2SC phases may coexist in a small region close to the chiral phase transition. For the CDW Ansatz, such a coexistence phase was already found in Refs. [21, 25] and here we confirm a coexistence phase also for the RKC Ansatz. Another result of this mean-field study is that the specific shape of the inhomogeneous chiral condensate impacts the size of the inhomogeneous region as well as the size of the coexistence region. It is known that the RKC Ansatz is energetically preferred over the CDW Ansatz [78] and therefore the inhomogeneous region is slightly larger for the CDW Ansatz.

This mean-field study can be extended in several ways. For instance, the model can be easily extended to include finite current quark masses, for which the RKC density of states is known as well [77, 111]. Furthermore, it should be possible to add further degrees of freedom, such as vector interactions [112] and Polyakov-loop dynamics [113]. Lastly, it would be also interesting to consider isospin-asymmetric matter $[26,114]$ especially in the 
context of neutron stars, where it is known that the electric neutrality and beta equilibrium lead to an isospin asymmetry.

In the second part of this thesis we studied the phase diagram of the QMD model including bosonic fluctuations. To this end, we employed the functional renormalization group in the local potential approximation and derived the flow equation of the effective potential and the static pion twopoint function for the QMD model. In the limit of zero diquark coupling the resulting phase diagram agrees very well with the one found in Ref. [1]. We can both confirm the existence of the negative entropy-density region and the emergence of an instability region. We have found that increasing the diquark coupling almost completely removes the negative-entropy region. Furthermore, the region of instability competes with the 2SC phase in a similar fashion as the competition we found in our mean-field study in the first part of this thesis. We showed that increasing the diquark coupling leads to a smaller instability region. We also observed that there is an overlap between the instability region and the 2SC phase hinting at a possible coexistence phase. However, we would like to underline that the stability analysis comes with a caveat, since the instabilities found only arise during the flow and vanish in the IR. The instability region thus depends on the regulator and therefore no conclusive statement about the existence of inhomogeneous phases in the QMD model can be made. The discovery of the instability region, however, serves as an important motivation to continue research in this direction.

The most straightforward extension of this thesis would be to study the robustness of the results found in this thesis by using different regulators, such as the exponential regulator. Such a study would give us a better sense of whether the instabilities are merely a regulator artifact or a feature of the QM(D) model at finite temperature and density. More elaborate continuations of this work could be to improve the truncation for the bosonic twopoint functions by taking into account the momentum dependence of the three- and four-point functions using the BMW approximation $[115,116]$ and by including a running wave-function renormalization $Z_{k}$. The latter has been done already in Ref. [69] but the investigation of instabilities were not part of that work.

In this thesis we have only looked at the static pion two-point function, however, it would also be interesting to conduct an analogous investigation for the diquark two-point function. If an instability in the 'diquark direction' could be found, then this would be a hint for an even richer phase diagram where the diquark condensate could be also spatially varying.

Finally, the most important extension would be to use an explicit inhomogeneous Ansatz for the chiral order parameter in the FRG framework. If one were able to find an inhomogeneous phase using this approach, it would be a more definitive result than our stability analysis. On the other hand, in case we do not find an inhomogeneous phase with this approach, this would not automatically exclude the existence of this phase, since it could be that another inhomogeneous Ansatz, which is energetically more favorable, is preferred over the homogeneous phase. However, the combination of both 
approaches, the stability analysis presented in this thesis and the explicit inhomogeneous Ansatz, most likely would yield an unambiguous result leading to a better understanding of the QCD phase diagram. 



\section{Appendix A}

\section{Notation and Conventions}

\section{A.1 Units}

In this thesis natural units are used:

$$
k_{\mathrm{B}}=c=\hbar=1 .
$$

\section{A.2 Minkowski and Euclidean Spacetime}

The invariant space-time distance with metric signature $(+1,-1,-1,-1)$

$$
d s^{2}=x_{\mu} x^{\mu}=d t^{2}-d x^{2}-d y^{2}-d z^{2}
$$

can be rewritten using the Euclidean metric

$$
d s^{2}=-d \tau^{2}-d x^{2}-d y^{2}-d z^{2}=-x_{\mu}^{\mathrm{E}} x_{\mu}^{\mathrm{E}}
$$

by performing a Wick rotation $t=-i \tau$. The d'Alembert operator then becomes

$$
\partial_{\mu} \partial^{\mu}=-\partial_{\tau} \partial_{\tau}-\partial_{i} \partial_{i}=-\partial_{\mu}^{\mathrm{E}} \partial_{\mu}^{\mathrm{E}} .
$$

Now let us see how a generic generating functional is affected by the Wick rotation:

$$
\begin{aligned}
& \mathcal{Z}=\int \mathcal{D} \phi \mathcal{D} \bar{\psi} \mathcal{D} \psi \exp \left[i \int \mathrm{d}^{4} x\left(\bar{\psi} i \not \partial \psi+\partial_{\mu} \phi \partial^{\mu} \phi-V(\phi, \psi)\right)\right] \\
& =\int \mathcal{D} \phi \mathcal{D} \bar{\psi} \mathcal{D} \psi \exp \left[i \int \mathrm{d}(-i \tau) \mathrm{d}^{3} \vec{x}\left(-\bar{\psi} \partial_{\tau} \gamma_{0} \psi+\bar{\psi} i \vec{\partial} \cdot \vec{\gamma} \psi-\partial_{\mu}^{\mathrm{E}} \phi \partial_{\mu}^{\mathrm{E}} \phi-V(\phi, \psi)\right)\right] \\
& =\int \mathcal{D} \phi \mathcal{D} \bar{\psi} \mathcal{D} \psi \exp \left[-\int \mathrm{d}^{4} x^{\mathrm{E}}\left(\bar{\psi} \partial_{\mu}^{\mathrm{E}} \gamma_{\mu}^{\mathrm{E}} \psi+\partial_{\mu}^{\mathrm{E}} \phi \partial_{\mu}^{\mathrm{E}} \phi+V(\phi, \psi)\right)\right] .
\end{aligned}
$$

In the last step we converted the gamma matrices from Minkowski to Euclidean coordinates:

$$
\gamma_{0}^{\mathrm{E}}=\gamma_{0}, \quad \gamma_{i}^{\mathrm{E}}=-i \gamma_{i}
$$


Throughout the thesis we will drop the label for the Euclidean coordinates, i.e., $x_{\mu}^{\mathrm{E}} \rightarrow x_{\mu}$. Euclidean four-vectors are always denoted with lower greek indices.

\section{A.3 Momentum Integral at Finite Temperature}

The Euclidean four-vector is given by $p \equiv p_{\mu}=\left(p_{0}, \vec{p}\right)^{\mathrm{T}}$. The zero component of the momentum four-vector, $p_{0}$, becomes the Matsubara frequency at finite temperature. The bosonic and fermionic Matsubara frequencies are defined as

$$
p_{0} \longrightarrow \omega_{n}=\left\{\begin{array}{ll}
2 n \pi T & \text { bosonic } \\
(2 n+1) \pi T & \text { fermionic }
\end{array} .\right.
$$

We also employ the following shorthand notation for integrals at finite temperature:

$$
\int_{x} \equiv \int_{0}^{1 / T} \mathrm{~d} \tau \int \mathrm{d}^{3} x, \quad \int_{p} \equiv T \sum_{n \in \mathbb{Z}} \int \frac{\mathrm{d}^{3} p}{(2 \pi)^{3}}
$$

Furthermore, the Dirac delta distribution at finite temperature is defined as

$$
\delta(q-p) \equiv(2 \pi)^{3} \delta^{(3)}(\vec{q}-\vec{p}) \delta_{n, m}
$$

\section{A.4 Dirac Algebra}

First, we define the Pauli matrices:

$$
\sigma_{1}=\left(\begin{array}{ll}
0 & 1 \\
1 & 0
\end{array}\right), \quad \sigma_{2}=\left(\begin{array}{cc}
0 & -i \\
i & 0
\end{array}\right), \quad \sigma_{3}=\left(\begin{array}{cc}
1 & 0 \\
0 & -1
\end{array}\right) .
$$

The Pauli matrices are Hermitian and from their product

$$
\sigma_{a} \sigma_{b}=\delta_{a b} \mathbb{1}+i \epsilon_{a b c} \sigma_{c},
$$

the commutation and anticommutation relations

$$
\left[\sigma_{a}, \sigma_{b}\right]=2 i \epsilon_{a b c} \sigma_{c}, \quad\left\{\sigma_{a}, \sigma_{b}\right\}=2 \delta_{a b} \mathbb{1},
$$

follow. The Euclidean gamma matrices in the chiral representation are defined as

$$
\gamma_{k}=\left(\begin{array}{cc}
0 & -i \sigma_{k} \\
i \sigma_{k} & 0
\end{array}\right), \quad \gamma_{0}=\left(\begin{array}{ll}
0 & \mathbb{1} \\
\mathbb{1} & 0
\end{array}\right), \quad \gamma_{5}=\gamma_{1} \gamma_{2} \gamma_{3} \gamma_{0}=\left(\begin{array}{cc}
\mathbb{1} & 0 \\
0 & -\mathbb{1}
\end{array}\right)
$$


where $k=1,2,3$. The Gamma matrices have the following properties:

$$
\left\{\gamma_{\mu} \gamma_{\nu}\right\}=\gamma_{\mu} \gamma_{\nu}+\gamma_{\nu} \gamma_{\mu}=2 \delta_{\mu v} \mathbb{1}_{4 \times 4}, \quad\left\{\gamma_{\mu} \gamma_{5}\right\}=0, \quad\left(\gamma_{\mu}\right)^{\dagger}=\gamma_{\mu}
$$





\section{Appendix B}

\section{Attractive Diquark Channel}

Quarks come in two possible spins, $N_{f}$ flavors (here: $N_{f}=2$ ) and $N_{c}=3$ colors, thus the wave function of a quark can be decomposed into the wave functions of the subspaces of these quantum numbers:

$$
|\psi\rangle=|c\rangle|f\rangle|s\rangle|j\rangle,
$$

where $|c\rangle$ is the color, $|f\rangle$ the flavor, $|s\rangle$ the spin, and $|j\rangle$ the orbital part of the total wave function. A diquark is a bound state of two quarks, therefore we have to combine the wave functions for two quarks:

$$
|\Delta\rangle=\left|\psi_{1} \psi_{2}\right\rangle=\left|c_{1} c_{2}\right\rangle\left|f_{1} f_{2}\right\rangle\left|j_{1} j_{2} ; s_{1} s_{2}\right\rangle .
$$

If we want to construct a diquark with total angular momentum $J=0$, the combined spin and orbital wave function must be antisymmetric, i.e., $\left|j_{1} j_{2} ; s_{1} s_{2}\right\rangle=1 / \sqrt{2}|\uparrow \downarrow-\downarrow \uparrow\rangle$. Furthermore, we demand that the color part of the wave function must be antisymmetric, because only the anti-triplet configuration leads to an attractive potential, which is a necessary condition for Cooper pairing. Finally, the wave function must then also be antisymmetric with respect to the flavor indices in order to respect Fermi-Dirac statistics, i.e., $\psi\left(x_{1}, x_{2}\right) \stackrel{!}{=}-\psi\left(x_{2}, x_{1}\right)$. These considerations yield the total wave functions for the three possible scalar spin-zero diquarks:

$$
\begin{aligned}
& \left|\Delta_{\bar{B}}\right\rangle=\frac{1}{\sqrt{2}^{3}}|R G-G R\rangle|u d-d u\rangle|\uparrow \downarrow-\downarrow \uparrow\rangle, \\
& \left|\Delta_{\bar{G}}\right\rangle=\frac{1}{\sqrt{2}^{3}}|R B-B R\rangle|u d-d u\rangle|\uparrow \downarrow-\downarrow \uparrow\rangle, \\
& \left|\Delta_{\bar{R}}\right\rangle=\frac{1}{\sqrt{2}^{3}}|G B-B G\rangle|u d-d u\rangle|\uparrow \downarrow-\downarrow \uparrow\rangle .
\end{aligned}
$$

In the next step we would like to show that the color anti-triplet configuration is indeed attractive at tree level. The flavor and angular momentum wave function part are irrelevant for the determination of the interaction, therefore we only consider the different color wave functions of the color anti-triplet configuration 


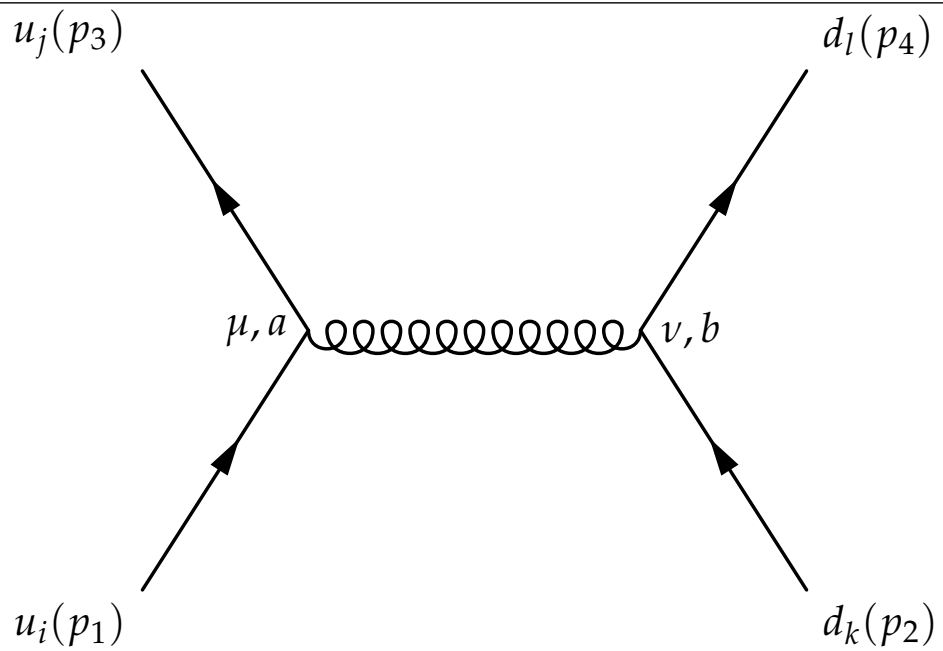

FIGURE B.1: Quark-quark tree-level scattering diagram. $i, j, k, l$ are the color components of the corresponding quarks, while $a, b$ are the color indices of the gluon propagator.

$$
\frac{|R G-G R\rangle}{\sqrt{2}}, \frac{|R B-B R\rangle}{\sqrt{2}}, \frac{|G B-B G\rangle}{\sqrt{2}} .
$$

In the following we use the Feynman rules in order to obtain the tree-level scattering amplitude of Fig. B.1:

$$
\begin{aligned}
& -i \mathcal{M}= \\
& {\left[\bar{u}_{u}\left(p_{3}\right) c_{j}^{\dagger}\left(-\frac{i}{2} g_{\mathrm{s}} \gamma^{\mu} \lambda^{a}\right) c_{i} u_{u}\left(p_{1}\right)\right] \frac{-g_{\mu v} \delta^{a b}}{q^{2}}\left[\bar{u}_{d}\left(p_{4}\right) c_{l}^{\dagger}\left(-\frac{i}{2} g_{\mathrm{s}} \gamma^{v} \lambda^{b}\right) c_{k} u_{d}\left(p_{2}\right)\right]} \\
& \equiv i g_{\mathrm{s}}^{2} C_{\mathrm{F}, i j k l}\left[\bar{u}_{u}\left(p_{3}\right) \gamma^{\mu} u_{u}\left(p_{1}\right)\right] \frac{g_{\mu v}}{q^{2}}\left[\bar{u}_{d}\left(p_{4}\right) \gamma^{v} u_{d}\left(p_{2}\right)\right]
\end{aligned}
$$

with the color factor $C_{F}$ defined as

$$
C_{\mathrm{F}, i j k l}=\frac{1}{4}\left(c_{j}^{\dagger} \lambda^{a} c_{i}\right)\left(c_{l}^{\dagger} \lambda^{a} c_{k}\right),
$$

where $c_{i}$ denotes the color part of the respective quark. Here we use the the basis vectors $c_{1}=(1,0,0)^{\mathrm{T}}=R, c_{2}=(0,1,0)^{\mathrm{T}}=G, c_{3}=(0,0,1)^{\mathrm{T}}=$ $B$. Note that the sign of the amplitude now depends on the sign of $C_{\mathrm{F}, i j k l}$. In the next step, we need to sum all diagrams that correspond to the antitriplet configuration. For the sake of simplicity, we will only consider the state $1 / \sqrt{2}|R G-G R\rangle$ :

$$
\begin{aligned}
C_{\mathrm{F}, \overline{3}_{c}} & \sim \frac{1}{4}\left[\left(R^{\mathrm{T}} \lambda^{a} R\right)\left(G^{\mathrm{T}} \lambda^{a} G\right)-\left(G^{\mathrm{T}} \lambda^{a} R\right)\left(R^{\mathrm{T}} \lambda^{a} G\right)\right] \\
& =-\frac{2}{3} .
\end{aligned}
$$


Finally, the one-gluon exchange potential for large energies can be approximated by

$$
V=C_{\mathrm{F}} \frac{\alpha_{\mathrm{s}}}{r},
$$

which means that for a negative color factor the interaction is attractive, while for a positive one the interaction becomes repulsive. Thus we conclude that the anti-triplet diquark configuration is attractive and therefore this type of diquark fulfills the necessary condition in order to form a colorsuperconducting phase.

Note that the direct product of two color triplets yields $3_{c} \oplus 3_{c}=\overline{3}_{c} \otimes 6_{c}$, i.e., an anti-triplet and a sextet. The sextet states are found to be

$$
\frac{|R G+G R\rangle}{\sqrt{2}}, \frac{|R B+B R\rangle}{\sqrt{2}}, \frac{|G B+B G\rangle}{\sqrt{2}},|R R\rangle,|G G\rangle,|B B\rangle .
$$

Let us also determine the color factor for a state of the color sextet, $1 / \sqrt{2}|R G+G R\rangle:$

$$
C_{\mathrm{F}, \overline{3}_{c}} \sim\left(R^{\mathrm{T}} \lambda^{a} R\right)\left(G^{\mathrm{T}} \lambda^{a} G\right)+\left(G^{\mathrm{T}} \lambda^{a} R\right)\left(R^{\mathrm{T}} \lambda^{a} G\right)=\frac{1}{6} .
$$

A positive color factor is obtained and therefore the interaction is repulsive for this channel. Thus, in leading order in perturbation theory, only the antitriplet configuration is a proper candidate to describe color superconductivity. 



\section{Appendix C}

\section{Mean-Field Study Appendices}

\section{C.1 Hubbard-Stratonovich Transformation}

Here we perform the Hubbard-Stratonovich transformation for the NJL model with diquarks. To this end, we write down the generating function of the theory:

$$
\begin{aligned}
\mathcal{Z}_{\mathrm{QMD}}= & \int \mathcal{D} \bar{\psi} \mathcal{D} \psi \mathcal{D} \bar{\psi}_{c} \mathcal{D} \psi_{c} \exp \int_{x}\left\{\bar{\psi}\left(i \gamma^{\mu} \partial_{\mu}+\mu \gamma_{0}\right) \psi+G\left[(\bar{\psi} \psi)^{2}+\left(\bar{\psi} i \gamma_{5} \vec{\tau} \psi\right)^{2}\right]\right. \\
& \left.+G_{\Delta}\left(\bar{\psi}_{c} i \gamma_{5} \tau_{2} \lambda_{a} \psi\right)\left(\bar{\psi} i \gamma_{5} \tau_{2} \lambda_{a} \psi_{c}\right)\right\} .
\end{aligned}
$$

Next, we need to apply the integration rule for $n$-dimensional Gaussian integrals:

$$
\int_{X^{n}} \mathrm{e}^{-\frac{1}{2} \vec{X}^{\mathrm{T}} \cdot A \vec{X}+\vec{X}^{T} \cdot \vec{J}}=\sqrt{\frac{(2 \pi)^{n}}{\operatorname{det} A}} \mathrm{e}^{\frac{1}{2} \vec{J}^{\mathrm{T}} \cdot A^{-1} \vec{J}} .
$$

Let us first consider the quark-quark channel as an example

$$
\begin{aligned}
\vec{X} & =\left(\Delta_{a} / 2, \Delta_{a}^{*} / 2\right)^{\mathrm{T}}, \\
\vec{J} & =\left(\left(\bar{\psi}_{c} i \gamma_{5} \tau_{2} \lambda_{a} \psi\right),\left(\bar{\psi} i \gamma_{5} \tau_{2} \lambda_{a} \psi_{c}\right)\right)^{\mathrm{T}}, \\
A^{-1} & =G_{\Delta}\left(\begin{array}{ll}
0 & 1 \\
1 & 0
\end{array}\right),
\end{aligned}
$$

For the scalar and pseudoscalar quark-antiquark channels it is analogous and actually easier since it is just a one-dimensional functional integral in field space. For the scalar-isoscalar channel we have 


$$
\begin{aligned}
X & =\sigma, \\
J & =\bar{\psi} \psi, \\
A^{-1} & =\frac{1}{G_{\Delta}},
\end{aligned}
$$

and for the pseudoscalar isotriplet we have

$$
\begin{aligned}
X & =\vec{\pi}, \\
J & =\bar{\psi} i \gamma_{5} \vec{\tau} \psi, \\
A^{-1} & =\frac{1}{G_{\Delta}} .
\end{aligned}
$$

The new generating functional up to a different normalization factor now reads

$$
\mathcal{Z}_{\mathrm{QMD}}=\int \mathcal{D} \bar{\psi} \mathcal{D} \psi \mathcal{D} \sigma \mathcal{D} \vec{\pi} \mathcal{D} \Delta_{A} \mathcal{D} \Delta_{A}^{*} \exp \int_{x} \mathcal{L}_{\text {eff }}
$$

with

$$
\begin{aligned}
\mathcal{L}_{\text {eff }}= & \bar{\psi}\left(i \not \partial+\mu \gamma_{0}+\sigma+i \gamma_{5} \vec{\pi} \cdot \vec{\tau}\right) \psi \\
& +\frac{\Delta_{A}}{2}\left(\bar{\psi}_{c} i \gamma_{5} \tau^{2} \lambda^{A} \psi\right)+\frac{\Delta_{A}^{*}}{2}\left(\bar{\psi} i \gamma_{5} \tau^{2} \lambda^{A} \psi_{c}\right)-\frac{\sigma^{2}+\vec{\pi}^{2}}{4 G}-\frac{\left|\Delta_{A}\right|^{2}}{4 G_{\Delta}} .
\end{aligned}
$$

Finally, we use the fermion-doubling trick [76] and obtain

$$
\begin{aligned}
\mathcal{L}_{\text {eff }}= & \frac{1}{2}\left[\bar{\psi}\left(i \not \partial+\mu \gamma_{0}+\sigma+i \gamma_{5} \vec{\pi} \cdot \vec{\tau}\right) \psi+\bar{\psi}_{c}\left(i \not \partial-\mu \gamma_{0}+\sigma+i \gamma_{5} \vec{\pi} \cdot \vec{\tau}\right) \psi_{c}\right. \\
& \left.+\Delta_{A}\left(\bar{\psi}_{c} i \gamma_{5} \tau^{2} \lambda^{A} \psi\right)+\Delta_{A}^{*}\left(\bar{\psi} i \gamma_{5} \tau^{2} \lambda^{A} \psi_{c}\right)-\frac{\sigma^{2}+\vec{\pi}^{2}}{2 G}-\frac{\left|\Delta_{A}\right|^{2}}{2 G_{\Delta}}\right] .
\end{aligned}
$$

\section{C.2 Trace Log}

We use the following property for the logarithm of a diagonalizable matrix:

$$
\ln A=V \ln \tilde{A} V^{-1},
$$

where $V$ is the transformation matrix consisting of the eigenvectors of $A$, which diagonalizes A. $\tilde{A}$ is the diagonalized matrix. We apply this identity 
to the kinetic part of the grand potential in Eq. (3.9) and use also the cyclicity of the Tr operator:

$$
\operatorname{Tr} \ln \left(\frac{\mathcal{S}^{-1}}{T}\right)=\operatorname{Tr}\left[V \ln \left(\frac{\tilde{\mathcal{S}}^{-1}}{T}\right) V^{-1}\right]=\operatorname{Tr} \ln \left(\frac{\tilde{\mathcal{S}}^{-1}}{T}\right) .
$$

Since the logarithm of the diagonal matrix $\tilde{\mathcal{S}}^{-1}$ is again a diagonal matrix, the trace is the sum that runs over all eigenvalues of $\ln \left(\frac{\mathcal{S}^{-1}}{T}\right)$. We therefore obtain

$$
\Omega_{\mathrm{kin}} \equiv-\frac{1}{2} \frac{T}{V} \operatorname{Tr} \ln \left(\frac{\mathcal{S}^{-1}}{T}\right)=-\frac{1}{2} \frac{1}{V} \sum_{n} \sum_{\lambda}\left[\ln \left(\frac{i \omega_{n}+\mathcal{E}_{\lambda}}{T}\right)\right]
$$

where $\mathcal{E}_{\lambda}$ are the eigenvalues of $H_{\mathrm{NG}}$.

\section{C.3 Square Hamiltonian}

We calculate the square Hamiltonian

$$
H_{\mathrm{NG}}^{2}=\left(\begin{array}{cc}
(H-\mu)^{2}-\gamma^{0} \hat{\Delta} \gamma^{0} \hat{\Delta}^{+} & -(H-\mu) \gamma^{0} \hat{\Delta}-\gamma^{0} \hat{\Delta}(H+\mu) \\
\gamma^{0} \hat{\Delta}^{+}(H-\mu)+(H+\mu) \gamma^{0} \hat{\Delta}^{+} & (H+\mu)^{2}-\gamma^{0} \hat{\Delta}^{+} \gamma^{0} \hat{\Delta}
\end{array}\right)
$$

Using $\left\{\gamma_{i}, \gamma_{0}\right\}=0$, we find that the off-diagonal elements vanish:

$$
-(H-\mu) \gamma^{0} \hat{\Delta}-\gamma^{0} \hat{\Delta}(H+\mu)=\gamma^{0} \hat{\Delta}^{\dagger}(H-\mu)+(H+\mu) \gamma^{0} \hat{\Delta}^{\dagger}=0 .
$$

On the other hand, the diagonal elements can be simplified to

$$
-\gamma^{0} \hat{\Delta} \gamma^{0} \hat{\Delta}^{\dagger}=-\gamma^{0} \hat{\Delta}^{\dagger} \gamma^{0} \hat{\Delta}=|\Delta|^{2} \lambda_{2}^{2}
$$

and thus we obtain

$$
H_{\mathrm{NG}}^{2}=\left(\begin{array}{cc}
(H-\mu)^{2}+|\Delta|^{2} \mathcal{P}_{r g} & 0 \\
0 & (H+\mu)^{2}+|\Delta|^{2} \mathcal{P}_{r g}
\end{array}\right) .
$$





\section{Appendix D}

\section{Flow of the Effective Potential}

The starting point for deriving the flow equations for the effective potential and the two-point function is the Wetterich equation given in Eq. (4.22):

$$
\begin{aligned}
\partial_{k} \Gamma_{k} & =\frac{1}{2} \operatorname{STr}\left[\partial_{k} R_{k}\left(\Gamma_{k}^{(2)}+R_{k}\right)^{-1}\right], \\
& =\frac{1}{2} \operatorname{Tr}\left[\partial_{k} R_{k, \mathrm{~B}}\left(\Gamma_{k, \mathrm{~B}}^{(2)}+R_{k, \mathrm{~B}}\right)^{-1}\right]-\frac{1}{2} \operatorname{Tr}\left[\partial_{k} R_{k, \mathrm{~F}}\left(\Gamma_{k, \mathrm{~F}}^{(2)}+R_{k, \mathrm{~F}}\right)^{-1}\right] .
\end{aligned}
$$

Here, we separated the Wetterich equation into the bosonic and fermionic contribution, since in our case the contributions from the bosonic and fermionic subspaces are disjoint.

As a reminder, the action of the QMD model is given as

$$
\begin{aligned}
& \Gamma_{\mathrm{QMD}}[\Phi]=\int d^{4} x\left\{\bar{\psi}\left[\not \partial-\mu \gamma_{0}+h_{\phi}\left(\sigma+i \gamma_{5} \vec{\tau} \cdot \vec{\pi}\right)\right] \psi\right. \\
& +h_{\Delta}\left[\Delta^{* a} \psi^{\mathrm{T}} C \tau_{2} \lambda^{a} \gamma_{5} \psi+\Delta^{a} \bar{\psi} \gamma_{5} \lambda^{a} \tau_{2} C \bar{\psi}^{\mathrm{T}}\right]+\frac{1}{2}\left[\left(\partial_{\mu} \sigma\right)^{2}+\left(\partial_{\mu} \vec{\pi}\right)^{2}\right] \\
& \left.+\frac{1}{2}\left[\left(\partial_{v}-2 \delta_{v 0} \mu\right) \Delta^{* a}\right]\left[\left(\partial_{v}+2 \delta_{v 0} \mu\right) \Delta^{a}\right]+U_{k}\left(\phi^{2}, \Delta^{2}\right)-c \sigma\right\} .
\end{aligned}
$$

The flow equation for the effective potential is obtained by the following projection:

$$
\begin{aligned}
\left.\frac{1}{\operatorname{vol}_{d}} \Gamma_{k}\right|_{\psi=\bar{\psi}=0, \phi=\sigma, \Delta_{i}=\delta_{i 2} \Delta} & \equiv V_{k}\left(\sigma^{2}, \Delta^{2}\right) \\
& =U_{k}\left(\sigma^{2}, \Delta^{2}\right)-c \sigma-2 \mu^{2} \Delta^{2}
\end{aligned}
$$

The explicit symmetry breaking term $-c \sigma$ and the diquark mass term $-2 \mu^{2} \Delta^{2}$ are scale independent in our truncation and therefore they can just be added to the effective potential in the IR after solving the flow equation for $U_{k}$.

\section{D.1 Bosonic Contribution}

In general $\Gamma_{k}^{(2)}$ in the bosonic field space looks like this: 


$$
\begin{aligned}
& \Gamma_{k, \mathrm{~B}}^{(2)}=
\end{aligned}
$$

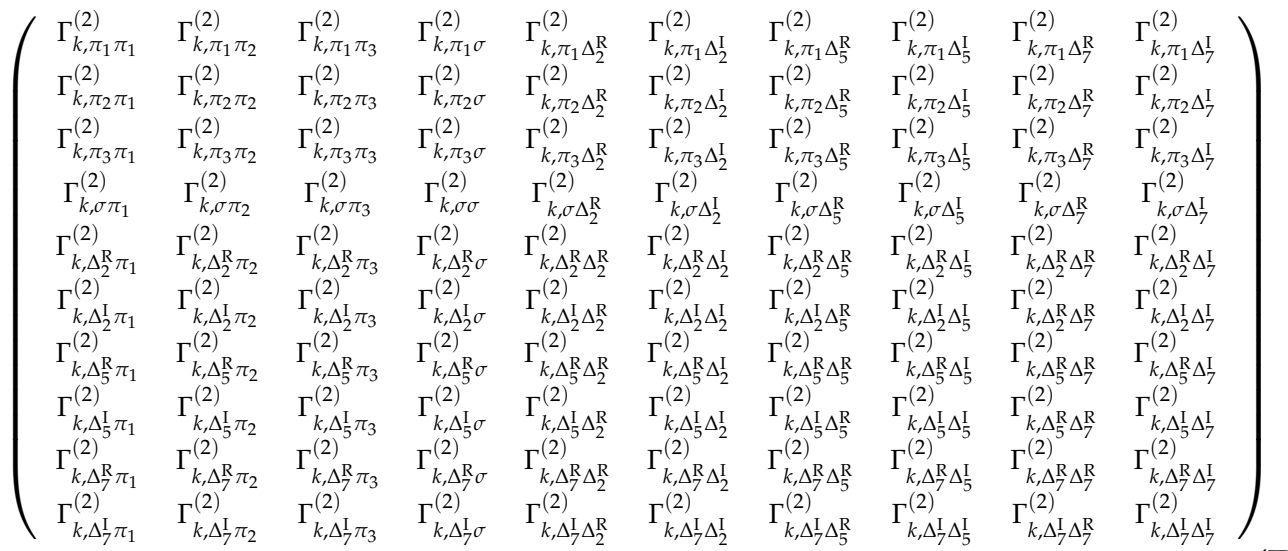

Here we choose the real and imaginary parts of the diquark as independent degrees of freedom. $\Delta_{i}^{\mathrm{R}}$ denotes the real part of the $i$-th diquark and $\Delta_{i}^{\mathrm{I}}$ the imaginary part. The index runs over $i=2,5,7$ corresponding to the different antisymmetric Gell-Mann matrices. Most entries of Eq. (D.4) vanish and three distinct subspaces can be identified:

$$
\Gamma_{k, B}^{(2)}\left(\pi, \sigma, \Delta_{2}, \Delta_{5}, \Delta_{7}\right)=\left(\begin{array}{ccc}
\Gamma_{k, \pi}^{(2)} & 0 & 0 \\
0 & \Gamma_{k, \sigma-\Delta}^{(2)} & 0 \\
0 & 0 & \Gamma_{k, \Delta}^{(2)}
\end{array}\right)
$$

The submatrices are found to be

$$
\begin{aligned}
& \Gamma_{k, \pi}^{(2)}=\left(\begin{array}{ccc}
p^{2}+2 U_{k, \sigma} & 0 & 0 \\
0 & p^{2}+2 U_{k, \sigma} & 0 \\
0 & 0 & p^{2}+2 U_{k, \sigma}
\end{array}\right) \\
& \Gamma_{k, \sigma-\Delta}^{(2)}=\left(\begin{array}{ccc}
p^{2}+2 U_{k, \sigma}+4 \sigma^{2} U_{k, \sigma \sigma} & 4 \Delta \sigma U_{k, \sigma \Delta} & 0 \\
4 \Delta \sigma U_{k, \sigma \Delta} & 4 \Delta^{2} U_{k, \Delta \Delta}+2 U_{k, \Delta}-4 \mu^{2}+p^{2} & 4 \mu p_{0} \\
0 & -4 \mu p_{0} & 2 U_{k, \Delta}-4 \mu^{2}+p^{2}
\end{array}\right), \\
& \Gamma_{k, \Delta}^{(2)}=\left(\begin{array}{cccc}
2 U_{k, \Delta}-4 \mu^{2}+p^{2} & 4 \mu p_{0} & 0 & 0 \\
-4 \mu p_{0} & 2 U_{k, \Delta}-4 \mu^{2}+p^{2} & 0 & 0 \\
0 & 0 & 2 U_{k, \Delta}-4 \mu^{2}+p^{2} & 4 \mu p_{0} \\
0 & 0 & -4 \mu p_{0} & 2 U_{k, \Delta}-4 \mu^{2}+p^{2}
\end{array}\right) .
\end{aligned}
$$

The derivatives of the effective potential are defined as 


$$
\begin{aligned}
U_{k, \sigma} & =\frac{\partial U_{k}}{\partial \sigma^{2}}, \\
U_{k, \sigma \sigma} & =\frac{\partial^{2} U_{k}}{\partial \sigma^{2} \partial \sigma^{2}}, \\
U_{k, \Delta} & =\frac{\partial U_{k}}{\partial \Delta^{2}}, \\
U_{k, \Delta \Delta} & =\frac{\partial^{2} U_{k}}{\partial \Delta^{2} \partial \Delta^{2}}, \\
U_{k, \sigma \Delta} & =U_{k, \Delta \sigma}=\frac{\partial^{2} U_{k}}{\partial \sigma^{2} \partial \Delta^{2}} .
\end{aligned}
$$

As bosonic regulator we choose the three-dimensional analogue of the optimized Litim regulators [94]:

$$
R_{k, \mathrm{~B}}=\left(k^{2}-\vec{p}^{2}\right) \theta\left(k^{2}-\vec{p}^{2}\right)
$$

We find the inverse of $\left(\Gamma_{k, \mathrm{~B}}^{(2)}+R_{k, \mathrm{~B}} \mathbb{1}_{10 \times 10}\right)$ by inverting the three submatrices:

$$
\begin{aligned}
& \left(\Gamma_{k, \pi}^{(2)}+R_{k, \mathrm{~B}} \mathbb{1}_{3 \times 3}\right)^{-1}=\left(\begin{array}{ccc}
1 / E_{\pi}^{2} & 0 & 0 \\
0 & 1 / E_{\pi}^{2} & 0 \\
0 & 0 & 1 / E_{\pi}^{2}
\end{array}\right) \\
& \left(\Gamma_{k, \sigma-\Delta}^{(2)}+R_{k, \mathrm{~B}} \mathbb{1}_{3 \times 3}\right)^{-1}= \\
& \frac{1}{16 \mu^{2} p_{0}^{2} E_{\sigma}^{2}+E_{\Delta, 0}^{2}\left(E_{\Delta}^{2} E_{\sigma}^{2}-16 \Delta^{2} \sigma^{2} U_{k, \sigma \Delta}^{2}\right)} \\
& \left(\begin{array}{ccc}
E_{\Delta}^{2} E_{\Delta, 0}^{2}+16 p_{0}^{2} \mu^{2} & -4 \Delta \sigma U_{k, \sigma \Delta} E_{\Delta, 0}^{2} & 16 \mu p_{0} \Delta \sigma U_{k, \sigma \Delta} \\
-4 \Delta \sigma U_{k, \sigma \Delta} E_{\Delta, 0}^{2} & E_{\Delta, 0}^{2} E_{\sigma}^{2} & -4 \mu p_{0} E_{\sigma}^{2} \\
-16 \mu p_{0} \Delta \sigma U_{k, \sigma \Delta} & 4 \mu p_{0} E_{\sigma}^{2} & E_{\Delta}^{2} E_{\sigma}^{2}-16 \Delta^{2} \sigma^{2} U_{k, \sigma \Delta}^{2}
\end{array}\right), \\
& \left(\Gamma_{k, \Delta}^{(2)}+R_{k, \mathrm{~B}} \mathbb{1}_{4 \times 4}\right)^{-1}=\frac{1}{E_{\Delta, 0}^{4}+16 \mu^{2} p_{0}^{2}}\left(\begin{array}{cccc}
E_{\Delta, 0}^{2} & -4 \mu p_{0} & 0 & 0 \\
4 \mu p_{0} & E_{\Delta, 0}^{2} & 0 & 0 \\
0 & 0 & E_{\Delta, 0}^{2} & -4 \mu p_{0} \\
0 & 0 & 4 \mu p_{0} & E_{\Delta, 0}^{2}
\end{array}\right) .
\end{aligned}
$$

For a more compact notation we defined 


$$
E_{x}=\sqrt{R_{k, \mathrm{~B}}+p^{2}+m_{x}^{2}}=\sqrt{R_{k, \mathrm{~B}}+p_{0}^{2}+\vec{p}^{2}+m_{x}^{2}}
$$

where

$$
\begin{aligned}
m_{\pi} & =\sqrt{2 U_{k, \sigma}}, \\
m_{\sigma} & =\sqrt{2 U_{k, \sigma}+4 \sigma^{2} U_{k, \sigma \sigma}}, \\
m_{\Delta, 0} & =\sqrt{2 U_{k, \Delta}-4 \mu^{2}}, \\
m_{\Delta} & =\sqrt{2 U_{k, \Delta}+4 \Delta^{2} U_{k, \Delta \Delta}-4 \mu^{2}} .
\end{aligned}
$$

Since the submatrices do not mix with each other, the Wetterich equation further decomposes into three parts:

$$
\partial_{k} \Gamma_{k, \mathrm{~B}}=\frac{1}{2}\left[\operatorname{Tr}\left(\frac{\partial_{k} R_{k, \mathrm{~B}}}{\Gamma_{k, \pi}^{(2)}+R_{k, \mathrm{~B}}}\right)+\operatorname{Tr}\left(\frac{\partial_{k} R_{k, \mathrm{~B}}}{\Gamma_{k, \sigma-\Delta}^{(2)}+R_{k, \mathrm{~B}}}\right)+\operatorname{Tr}\left(\frac{\partial_{k} R_{k, \mathrm{~B}}}{\Gamma_{k, \Delta}^{(2)}+R_{k, \mathrm{~B}}}\right)\right] .
$$

The scale derivative of the bosonic regulator is found to be

$$
\begin{aligned}
\partial_{k} R_{k, \mathrm{~B}} & =\partial_{k}\left(k^{2}-\vec{p}^{2}\right) \theta\left(k^{2}-\vec{p}^{2}\right)=2 k \theta\left(k^{2}-\vec{p}^{2}\right)+2 k\left(k^{2}-\vec{p}^{2}\right) \delta\left(k^{2}-\vec{p}^{2}\right) \\
& =2 k \theta\left(k^{2}-\vec{p}^{2}\right) .
\end{aligned}
$$

The contribution with the delta distribution always vanishes in our calculations, therefore we omit it here. The trace is understood as the following operation:

$$
\operatorname{Tr}=V T \sum_{n \in \mathbb{Z}} \int \frac{\mathrm{d}^{3} p}{(2 \pi)^{3}} \operatorname{tr}
$$

where tr is the trace over all remaining spaces, i.e., field, flavor, color, and Dirac space. Furthermore, we need to replace the zero momenta by the bosonic Matsubara frequencies: $p_{0} \rightarrow \omega_{n}=2 \pi T n$. Let us first calculate the pion contribution: 


$$
\begin{gathered}
\operatorname{Tr}\left(\frac{\partial_{k} R_{k, \mathrm{~B}}}{\Gamma_{k, \pi}^{(2)}+R_{k, \mathrm{~B}}}\right)=V T \sum_{n \in \mathbb{Z}} \int \frac{\mathrm{d}^{3} p}{(2 \pi)^{3}} \operatorname{tr}\left(\frac{\partial_{k} R_{k, \mathrm{~B}}}{\Gamma_{k, \pi}^{(2)}+R_{k, \mathrm{~B}}}\right) \\
=V T \sum_{n \in \mathbb{Z}} \int \frac{\mathrm{d}^{3} p}{(2 \pi)^{3}} 2 k \theta\left(k^{2}-\vec{p}^{2}\right) \operatorname{tr}\left(\frac{1}{\Gamma_{k, \pi}^{(2)}+R_{k, \mathrm{~B}}}\right) \\
=\frac{4 \pi V}{(2 \pi)^{3}} T \sum_{n \in \mathbb{Z}} \int_{0}^{\infty} \mathrm{d} p p^{2} 2 k \theta\left(k^{2}-\vec{p}^{2}\right) \operatorname{tr}\left(\begin{array}{ccc}
1 / E_{\pi}^{2} & 0 & 0 \\
0 & 1 / E_{\pi}^{2} & 0 \\
0 & 0 & 1 / E_{\pi}^{2}
\end{array}\right) .
\end{gathered}
$$

In the last line we switched to spherical coordinates, where the angular integration could already be evaluated and yields the factor $4 \pi$. Because of the Heaviside function, only contributions up to $p \leq k$ will be integrated. Then, the Heaviside function in the energy functions can be dropped and $E_{x}$ becomes

$$
\begin{aligned}
E_{x} & =\sqrt{R_{k, \mathrm{~B}}+p_{0}^{2}+\vec{p}^{2}+m_{x}^{2}}=\sqrt{k^{2}-\vec{p}^{2}+p_{0}^{2}+\vec{p}^{2}+m_{x}^{2}}=\sqrt{p_{0}^{2}+k^{2}+m_{x}^{2}} \\
& \equiv \sqrt{\omega_{n}^{2}+E_{x, k}^{2}} .
\end{aligned}
$$

The trace in field space simply yields a factor of 3 , thus we obtain

$$
\begin{aligned}
& =\frac{4 \pi V}{(2 \pi)^{3}} T \sum_{n \in \mathbb{Z}} \int_{0}^{k} \mathrm{~d} p p^{2} 2 k \frac{3}{\omega_{n}^{2}+E_{\pi, k}^{2}} \\
& =\frac{3 \cdot 4 \pi V}{(2 \pi)^{3}} \frac{2 k^{4}}{3} T \sum_{n \in \mathbb{Z}} \frac{1}{\omega_{n}^{2}+E_{\pi, k}^{2}} \\
& =\frac{3 \cdot 4 \pi V}{(2 \pi)^{3}} \frac{2 k^{4}}{3} T \frac{\operatorname{coth} \frac{E_{\pi, k}}{2 T}}{2 E_{\pi, k} T} \\
& =\frac{V}{2 \pi^{2}} \frac{k^{4}}{E_{\pi, k}} \operatorname{coth} \frac{E_{\pi, k}}{2 T} .
\end{aligned}
$$

From the first to the second line we performed the momentum integral, and from the second to the third line we evaluated the Matsubara sum using standard techniques. The remaining bosonic contributions can be evaluated analogously. In the following we define $\tilde{E}_{\Delta, 0, k}=\sqrt{k^{2}+\tilde{m}_{\Delta, 0}} \equiv \sqrt{k^{2}+2 U_{k, \Delta}}$ and $\tilde{E}_{\Delta, k}=\sqrt{k^{2}+\tilde{m}_{\Delta}} \equiv \sqrt{k^{2}+2 U_{k, \Delta}+4 \Delta^{2} U_{k, \Delta \Delta}}$. For the ungapped contribution we obtain: 


$$
\begin{aligned}
& \operatorname{Tr}\left(\frac{\partial_{k} R_{k, \mathrm{~B}}}{\Gamma_{\Delta, 0, k}^{(2)}+R_{k, \mathrm{~B}}}\right) \\
& \quad=V T \sum_{n \in \mathbb{Z}} \int \frac{\mathrm{d}^{3} p}{(2 \pi)^{3}} \frac{1}{E_{\Delta, 0}^{4}+16 \mu^{2} \omega_{n}^{2}} \operatorname{tr}\left(\begin{array}{cccc}
E_{\Delta, 0}^{2} & -4 \mu \omega_{n} & 0 & 0 \\
4 \mu \omega_{n} & E_{\Delta, 0}^{2} & 0 & 0 \\
0 & 0 & E_{\Delta, 0}^{2} & -4 \mu \omega_{n} \\
0 & 0 & 4 \mu \omega_{n} & E_{\Delta, 0}^{2}
\end{array}\right) \\
& =\frac{4 \cdot 4 \pi V}{(2 \pi)^{3}} \frac{2 k^{4}}{3} T \sum_{n \in \mathbb{Z}} \frac{\omega_{n}^{2}+E_{\Delta, 0, k}^{2}}{\left(\omega_{n}^{2}+E_{\Delta, 0, k}^{2}\right)^{2}+16 \mu^{2} \omega_{n}^{2}} \\
& =\frac{4 \cdot 4 \pi V}{(2 \pi)^{3}} \frac{2 k^{4}}{3} \frac{1}{2 \tilde{E}_{\Delta, 0, k}} \frac{\sinh \frac{\tilde{E}_{\Delta, 0, k}}{T}}{\cosh \frac{\tilde{E}_{\Delta, 0, k}}{T}-\cosh \frac{2 \mu}{T}} \\
& =\frac{2 V}{3 \pi^{2}} \frac{k^{4}}{\tilde{E}_{\Delta, 0, k}} \frac{\sinh \frac{\tilde{E}_{\Delta, 0, k}}{T}}{\cosh \frac{\tilde{E}_{\Delta, 0, k}}{T}-\cosh \frac{2 \mu}{T}} \\
& =\frac{V}{3 \pi^{2}} \frac{k^{4}}{\tilde{E}_{\Delta, 0, k}}\left[\operatorname{coth} \frac{\tilde{E}_{\Delta, 0, k}-2 \mu}{2 T}+\operatorname{coth} \frac{\tilde{E}_{\Delta, 0, k}+2 \mu}{2 T}\right] .
\end{aligned}
$$

Note that we have a singularity for $\tilde{E}_{\Delta, 0, k}=2 \mu$. During the flow of the effective potential it might happen that the energy approaches the singularity, making the partial differential equation very stiff. The Matsubara sum for the second contribution of Eq. (D.26) is somewhat more complicated so we will have a look at this with a little more detail: 


$$
\begin{aligned}
& \operatorname{Tr}\left(\frac{\partial_{k} R_{k, \mathrm{~B}}}{\Gamma_{k, \sigma-\Delta}^{(2)}+R_{k, \mathrm{~B}}}\right) \\
& =V T \sum_{n \in \mathbb{Z}} \int \frac{\mathrm{d}^{3} p}{(2 \pi)^{3}} \frac{1}{16 \mu^{2} p_{0}^{2} E_{\sigma}^{2}+E_{\Delta, 0}^{2}\left(E_{\Delta}^{2} E_{\sigma}^{2}-16 \Delta^{2} \sigma^{2} U_{k, \sigma \Delta}^{2}\right)} \\
& \times\left(\begin{array}{ccc}
E_{\Delta}^{2} E_{\Delta, 0}^{2}+16 \omega_{n}^{2} \mu^{2} & -4 \Delta \sigma U_{k, \sigma \Delta} E_{\Delta, 0}^{2} & 16 \mu \omega_{n} \Delta \sigma U_{k, \sigma \Delta} \\
-4 \Delta \sigma U_{k, \sigma \Delta} E_{\Delta, 0}^{2} & E_{\Delta, 0}^{2} E_{\sigma}^{2} & -4 \mu \omega_{n} E_{\sigma}^{2} \\
-16 \mu \omega_{n} \Delta \sigma U_{k, \sigma \Delta} & 4 \mu \omega_{n} E_{\sigma}^{2} & E_{\Delta}^{2} E_{\sigma}^{2}-16 \Delta^{2} \sigma^{2} U_{k, \sigma \Delta}^{2}
\end{array}\right) \\
& =\frac{4 \pi V}{(2 \pi)^{3}} \frac{2 k^{4}}{3} T \sum_{n \in \mathbb{Z}} \\
& \times\left\{\frac{\left(\omega_{n}^{2}+E_{\Delta, k}^{2}\right)\left(\omega_{n}^{2}+E_{\Delta, 0, k}^{2}\right)+16 \omega_{n}^{2} \mu^{2}+\left(\omega_{n}^{2}+E_{\Delta, 0, k}^{2}\right)\left(\omega_{n}^{2}+E_{\sigma, k}^{2}\right)}{16 \mu^{2} \omega_{n}^{2}\left(\omega_{n}^{2}+E_{\sigma, k}^{2}\right)+\left(\omega_{n}^{2}+E_{\Delta, 0, k}^{2}\right)\left[\left(\omega_{n}^{2}+E_{\Delta, k}^{2}\right)\left(\omega_{n}^{2}+E_{\sigma, k}^{2}\right)-16 \Delta^{2} \sigma^{2} U_{k, \sigma \Delta}^{2}\right]}\right. \\
& \left.+\frac{\left(\omega_{n}^{2}+E_{\Delta, k}^{2}\right)\left(\omega_{n}^{2}+E_{\sigma, k}^{2}\right)-16 \Delta^{2} \sigma^{2} U_{k, \sigma \Delta}^{2}}{16 \mu^{2} \omega_{n}^{2}\left(\omega_{n}^{2}+E_{\sigma, k}^{2}\right)+\left(\omega_{n}^{2}+E_{\Delta, 0, k}^{2}\right)\left[\left(\omega_{n}^{2}+E_{\Delta, k}^{2}\right)\left(\omega_{n}^{2}+E_{\sigma, k}^{2}\right)-16 \Delta^{2} \sigma^{2} U_{k, \sigma \Delta}^{2}\right]}\right\}
\end{aligned}
$$

Note that the denominators of both terms in the last expression are the same. In the next step, we express the denominators in terms of its zeros with respect to $\omega_{n}{ }^{1}$. The sum can then be written as

$$
\begin{aligned}
=T \sum_{n \in \mathbb{Z}} \frac{\alpha_{0}+\alpha_{1} \omega_{n}^{2}+3\left(\omega_{n}^{2}\right)^{2}}{\beta_{0}+\beta_{1} \omega_{n}^{2}+\beta_{2}\left(\omega_{n}^{2}\right)^{2}+\left(\omega_{n}^{2}\right)^{3}} & =T \sum_{n \in \mathbb{Z}} \frac{\alpha_{0}+\alpha_{1} \omega_{n}^{2}+3\left(\omega_{n}^{2}\right)^{2}}{\left(\omega_{n}^{2}-r_{1}^{2}\right)\left(\omega_{n}^{2}-r_{2}^{2}\right)\left(\omega_{n}^{2}-r_{3}^{2}\right)} \\
& =\frac{1}{2} \sum_{i=1}^{3} \frac{\alpha_{0}-\alpha_{1} z_{i}^{2}+3 z_{i}^{4}}{\left(z_{i+1}^{2}-z_{i}^{2}\right)\left(z_{i+1}^{2}-z_{i}^{2}\right) z_{i}} \operatorname{coth} \frac{z_{i}}{2 T} .
\end{aligned}
$$

In the last step we used the residue theorem in order to derive the Matsubara sum. The coefficients are identified to be

$$
\begin{aligned}
& \alpha_{0}=E_{\Delta, k}^{2} E_{\Delta, 0, k}^{2}+E_{\Delta, k}^{2} E_{\sigma, k}^{2}+E_{\Delta, 0, k}^{2} E_{\sigma, k}^{2}-16 \sigma^{2} \Delta^{2} U_{k, \sigma \Delta}^{2}, \\
& \alpha_{1}=2\left(E_{\Delta, k}^{2}+E_{\Delta, 0, k}^{2}+E_{\sigma, k}^{2}+8 \mu^{2}\right), \\
& \beta_{0}=E_{\Delta, 0, k}^{2}\left(E_{\Delta, k}^{2} E_{\sigma, k}^{2}-\sigma^{2} \Delta^{2} U_{k, \sigma \Delta}^{2}\right), \\
& \beta_{1}=\alpha_{0}+16 E_{\sigma, k}^{2} \mu^{2}, \\
& \beta_{2}=E_{\Delta, k}^{2}+E_{\Delta, 0, k}^{2}+E_{\sigma, k}^{2}+16 \mu^{2} .
\end{aligned}
$$

\footnotetext{
${ }^{1}$ In general this works only for polynomials up to order $\left(\omega_{n}^{2}\right)^{4}$, for polynomials of higher order it may not be possible to determine the zeroes analytically.
} 
$z_{i}=i r_{i}$ are the roots of the denominator, and $z_{4}=z_{1}, z_{5}=z_{2}, z_{6}=z_{3}$. They can be looked up in literature as these are the roots of a third degree polynomial. The explicit expressions for these are

$$
r_{k+1}=-i z_{k+1}=-\frac{1}{3}\left(\beta_{2}+\eta^{i} C+\frac{b_{0}}{\eta^{i} C}\right), \quad k \in\{0,1,2\},
$$

where

$$
\begin{aligned}
& C=\sqrt[3]{\frac{b_{1} \pm \sqrt{b_{1}^{2}-4 b_{0}^{3}}}{2}} \\
& \eta=\frac{-1+\sqrt{-3}}{2}
\end{aligned}
$$

and

$$
\begin{aligned}
& b_{0}=\beta_{2}^{2}-3 \beta_{1} \\
& b_{1}=2 \beta_{2}^{3}-9 \beta_{2} \beta_{1}+27 \beta_{0} .
\end{aligned}
$$

Note that both choices for the sign in Eq. (D.41) lead to the same result as long as $C \neq 0$, which is the case for our purpose.

Finally, the final result for this contribution is given by

$$
\operatorname{Tr}\left(\frac{\partial_{k} R_{k, \mathrm{~B}}}{\Gamma_{k, \sigma-\Delta}^{(2)}+R_{k, \mathrm{~B}}}\right)=\frac{V}{\pi^{2}} \frac{k^{4}}{6} \sum_{i=1}^{3} \frac{\alpha_{0}-\alpha_{1} z_{i}^{2}+3 z_{i}^{4}}{\left(z_{i+1}^{2}-z_{i}^{2}\right)\left(z_{i+1}^{2}-z_{i}^{2}\right) z_{i}} \operatorname{coth} \frac{z_{i}}{2 T} .
$$

Now, we can add up all partial contributions in Eq. (D.26) and obtain

$$
\begin{aligned}
\partial_{k} U_{k, \mathrm{~B}}= & \frac{k^{4}}{12 \pi^{2}}\left[\frac{3}{E_{\pi, k}} \operatorname{coth} \frac{E_{\pi, k}}{2 T}+\sum_{i=1}^{3} \frac{\alpha_{0}-\alpha_{1} z_{i}^{2}+3 z_{i}^{4}}{\left(z_{i+1}^{2}-z_{i}^{2}\right)\left(z_{i+1}^{2}-z_{i}^{2}\right) z_{i}} \operatorname{coth} \frac{z_{i}}{2 T}\right. \\
& \left.+\frac{2}{\tilde{E}_{\Delta, 0, k}}\left(\operatorname{coth} \frac{\tilde{E}_{\Delta, 0, k}-2 \mu}{2 T}+\operatorname{coth} \frac{\tilde{E}_{\Delta, 0, k}+2 \mu}{2 T}\right)\right] .
\end{aligned}
$$

\section{D.2 Fermionic Contribution}

The fermionic contribution to the Wetterich equation is given as:

$$
\partial_{k} \Gamma_{k}=-\frac{1}{2} \operatorname{Tr}\left(\frac{\partial_{k} R_{k, \mathrm{~F}}}{\Gamma_{k, \mathrm{~F}}^{(2)}+R_{k, \mathrm{~F}}}\right)
$$


We choose the following basis for the fermion fields:

$$
\begin{gathered}
\psi=\left(\begin{array}{c}
\psi_{\mathrm{r}} \\
i \tau_{2} C \bar{\psi}_{\mathrm{g}}^{\mathrm{T}} \\
\psi_{\mathrm{b}}
\end{array}\right), \quad \bar{\psi}=\left(\begin{array}{c}
\bar{\psi}_{\mathrm{r}} \\
-i \psi_{\mathrm{g}}^{\mathrm{T}} C \tau_{2} \\
\bar{\psi}_{\mathrm{b}}
\end{array}\right), \\
\Psi=\left(\begin{array}{c}
\psi \\
\bar{\psi}^{\mathrm{T}}
\end{array}\right), \quad \bar{\Psi}=\left(\begin{array}{c}
\bar{\psi} \\
\psi^{\mathrm{T}}
\end{array}\right) .
\end{gathered}
$$

For the first two components the choice (D.48) is reminiscient of the NambuGorkov basis with color components explicitly given. This has the advantage that we can separate the resulting inverse propagator in the gapped and ungapped contributions more easily.

The fermionic two-point function in this basis reads

$$
\Gamma_{k, \mathrm{~F}}^{(2)}=\left(\begin{array}{cc}
\Gamma_{k, \bar{\psi} \psi}^{(2)} & \Gamma_{k, \bar{\psi} \bar{\psi}^{\mathrm{T}}}^{(2)} \\
\Gamma_{k, \psi^{\mathrm{T}} \psi}^{(2)} & \Gamma_{k, \psi^{\mathrm{T}} \bar{\psi}^{\mathrm{T}}}^{(2)}
\end{array}\right) .
$$

In the following we will already neglect the diquark contributions for the quarks that do not participate in the pairing, i.e., $\Delta_{5}=\Delta_{7}=0$. Therefore, the off-diagonal components $\Gamma_{k, \bar{\psi} \bar{\psi}^{\mathrm{T}}}^{(2)}$ and $\Gamma_{k, \psi^{\mathrm{T}} \psi}^{(2)}$ vanish, since these only contain contributions $\sim \Delta_{5 / 7}$. This is valid as long as we do not need to consider the three-point functions $\Gamma_{k, \bar{\psi} \psi \Delta_{5 / 7}}^{(2,1)}$, which is the case for this study. The remaining diagonal terms are then found to be

$$
\Gamma_{k, \mathrm{~F}}^{(2)}=\left(\begin{array}{cc}
\Gamma_{k, \bar{\psi} \psi}^{(2)} & 0 \\
0 & -\left(\Gamma_{k, \bar{\psi} \psi}^{(2)}\right)^{\mathrm{T}}
\end{array}\right)
$$

where

$$
\begin{aligned}
\Gamma_{k, \bar{\psi} \psi}^{(2)} & =\left(\begin{array}{cc}
\Gamma_{k, \mathrm{rg}}^{(2)} & 0 \\
0 & \Gamma_{k, \mathrm{~b}}^{(2)}
\end{array}\right) \\
& =\left(\begin{array}{ccc}
-i \vec{p}-\mu \gamma_{0}+h_{\phi}\left(\sigma+i \gamma_{5} \vec{\tau} \cdot \vec{\pi}\right) & \\
-h_{\Delta} \Delta_{2} \gamma_{5} & -i \vec{p}+\mu \gamma_{0}+h_{\phi} \Delta_{5}^{*} \gamma_{5}\left(\sigma-i \gamma_{5} \vec{\tau} \cdot \vec{\pi}\right) & 0 \\
0 & 0 & -i \vec{p}-\mu \gamma_{0}+h_{\phi}\left(\sigma+i \gamma_{5} \vec{\tau} \cdot \vec{\pi}\right)
\end{array}\right) .
\end{aligned}
$$

The regulator is defined as 


$$
R_{k, \mathrm{~F}}=\left(\begin{array}{cc}
R_{k, \bar{\psi} \psi} & 0 \\
0 & R_{k, \psi^{\mathrm{T}} \bar{\psi}^{\mathrm{T}}}
\end{array}\right)=\left(\begin{array}{cc}
-i \vec{p} r_{k}(\vec{p}) \otimes \mathbb{1}_{3 \times 3} & 0 \\
0 & i \vec{p}^{\mathrm{T}} r_{k}(\vec{p}) \otimes \mathbb{1}_{3 \times 3}
\end{array}\right)
$$

with

$$
r_{k}(\vec{p})=\left(\sqrt{\frac{k^{2}}{\vec{p}^{2}}}-1\right) \theta\left(k^{2}-\vec{p}^{2}\right)
$$

and the scale derivative of the regulator is then given as

$$
\partial_{k} R_{k, \mathrm{~F}}=\left(\begin{array}{cc}
-i \vec{p} \partial_{k} r_{k}(\vec{p}) \otimes \mathbb{1}_{3 \times 3} & 0 \\
0 & i \vec{p}^{\mathrm{T}} \partial_{k} r_{k}(\vec{p}) \otimes \mathbb{1}_{3 \times 3}
\end{array}\right) .
$$

Now, the inverse FRG propagator in Eq. (D.47) can be written as

$$
\left(\Gamma_{k, \mathrm{~F}}^{(2)}+R_{k, \mathrm{~F}}\right)^{-1}=\left(\begin{array}{cc}
\left(\Gamma_{k, \bar{\psi} \psi}^{(2)}+R_{k, \bar{\psi} \psi}\right)^{-1} & 0 \\
0 & \left(\Gamma_{k, \psi^{\mathrm{T}} \bar{\psi}^{\mathrm{T}}}^{(2)}+R_{k, \psi^{\mathrm{T}} \bar{\psi}^{\mathrm{T}}}\right)^{-1}
\end{array}\right)
$$

such that the trace of Eq. (D.47) can be simplified to

$$
\begin{aligned}
& \operatorname{Tr} \frac{\partial_{k} R_{k, \mathrm{~F}}}{\Gamma_{k, \mathrm{~F}}^{(2)}+R_{k, \mathrm{~F}}}=\operatorname{Tr}\left(\begin{array}{cc}
\frac{-i \vec{p} \partial_{k} r_{k}(\vec{p}) \otimes \mathbb{1}_{3 \times 3}}{\Gamma_{k, \bar{\psi} \psi}^{(2)}+R_{k, \bar{\psi} \psi}} & 0 \\
0 & \left(\frac{-i \vec{p} \partial_{k} r_{k}(\vec{p}) \otimes \mathbb{1}_{3 \times 3}}{\Gamma_{k, \bar{\psi} \psi}^{(2)}+R_{k, \bar{\psi} \psi}}\right)^{\mathrm{T}}
\end{array}\right) \\
& =\operatorname{Tr}\left(\begin{array}{cc}
\frac{-i \vec{p} \partial_{k} r_{k}(\vec{p}) \otimes \mathbb{1}_{3 \times 3}}{\Gamma_{k, \bar{\psi} \psi}^{(2)}+R_{k, \bar{\psi} \psi}} & 0 \\
0 & \frac{-i \vec{p} \partial_{k} r_{k}(\vec{p}) \otimes \mathbb{1}_{3 \times 3}}{\Gamma_{k, \bar{\psi} \psi}^{(2)}+R_{k, \bar{\psi} \psi}}
\end{array}\right) \\
& =2 \operatorname{Tr} \frac{-i \vec{p} \partial_{k} r_{k}(\vec{p}) \otimes \mathbb{1}_{3 \times 3}}{\Gamma_{k, \bar{\psi} \psi}^{(2)}+R_{k, \bar{\psi} \psi}} .
\end{aligned}
$$

Now, using the decomposition into the red-green and the blue contributions from Eq. (D.52) the fermionic contribution to the flow of the effective potential can be written as 


$$
\begin{aligned}
\partial_{k} \Gamma_{k, \mathrm{~F}} & =-\frac{1}{2} \operatorname{Tr}\left(\frac{\partial_{k} R_{k, \mathrm{~F}}}{\Gamma_{k, \mathrm{~F}}^{(2)}+R_{k, \mathrm{~F}}}\right) \\
& =-\operatorname{Tr}\left(\frac{-i \vec{p} \partial_{k} r_{k}(\vec{p})}{\Gamma_{k, \mathrm{rg}}^{(2)}-i \vec{p} r_{k}(\vec{p}) \otimes \mathbb{1}_{2 \times 2}}\right)-\operatorname{Tr}\left(\frac{-i \vec{p} \partial_{k} r_{k}(\vec{p})}{\Gamma_{k, \mathrm{~b}}^{(2)}-i \vec{p} r_{k}(\vec{p})}\right) .
\end{aligned}
$$

Finally, let us first evaluate the red-green contribution of Eq. (D.58). For the constant field configuration $\sigma=\sigma_{0} \stackrel{\text { relabeled }}{\longrightarrow} \sigma, \vec{\pi}=\overrightarrow{0}, \operatorname{Re} \Delta_{2}=\Delta$ and $\operatorname{Im} \Delta_{2}=0$ we obtain

$$
\begin{aligned}
& \Gamma_{k, \mathrm{rg}}^{(2)}-i \vec{p} r_{k}(\vec{p}) \otimes \mathbb{1}_{2 \times 2} \\
& =\left(\begin{array}{cc}
-i \vec{p}\left[1+r_{k}(\vec{p})\right]-\left(\mu+i p_{0}\right) \gamma_{0}+h_{\phi} \sigma & h_{\Delta} \Delta \gamma_{5} \\
-h_{\Delta} \Delta \gamma_{5} & -i \vec{p}\left[1+r_{k}(\vec{p})\right]+\left(\mu-i p_{0}\right) \gamma_{0}+h_{\phi} \sigma
\end{array}\right),
\end{aligned}
$$

Note that this is the same fermionic two-point function as for the quarkmeson-diquark model for two-color QCD [66], therefore we expect to obtain the same result as in Ref. [66]. This two-point function can be inverted following Ref. [117]:

$$
\left(\Gamma_{k, \mathrm{rg}}^{(2)}-i \vec{p} r_{k}(\vec{p}) \otimes \mathbb{1}_{2 \times 2}\right)^{-1}=\left(\begin{array}{cc}
G^{+} & -G_{0}^{+} D^{-} G^{-} \\
-G_{0}^{-} D^{+} G^{+} & G^{-}
\end{array}\right),
$$

where the newly introduced quantities are defined as

$$
\begin{aligned}
G^{ \pm} & =\frac{\left(-i p_{0}-E \pm \mu\right) \Lambda^{+} \gamma_{0}}{-p_{0}^{2}-\left(E_{p}^{\mp}\right)^{2}}+\frac{\left(-i p_{0}+E \pm \mu\right) \Lambda^{-} \gamma_{0}}{-p_{0}^{2}-\left(E_{p}^{ \pm}\right)^{2}} \\
G_{0}^{ \pm} & =\frac{\Lambda^{\mp} \gamma_{0}}{-i p_{0}+E+\mu}+\frac{\Lambda^{ \pm} \gamma_{0}}{-i p_{0}-E+\mu} \\
D^{ \pm} & =\mp h_{\Delta} \gamma_{5} \Delta
\end{aligned}
$$

with

$$
\begin{aligned}
\Lambda^{ \pm} & =\frac{1}{2}\left[1 \pm \frac{\gamma_{0}}{E}\left(-i \vec{p}\left(1+r_{k}\right)+h_{\phi} \sigma\right)\right], \\
E & =\sqrt{p^{2}\left(1+r_{k}\right)^{2}+\left(h_{\phi} \sigma\right)^{2}} \\
E_{p}^{ \pm} & =\sqrt{(E \pm \mu)^{2}+\left(h_{\Delta} \Delta\right)^{2}} .
\end{aligned}
$$

Now, let us evaluate the full trace for the red-green contribution: 


$$
\begin{aligned}
& \operatorname{Tr}\left(\frac{-i \vec{p} \partial_{k} r_{k}(\vec{p})}{\Gamma_{k, \mathrm{rg}}^{(2)}-i \vec{p} r_{k} \otimes \mathbb{1}_{2 \times 2}}\right) \\
& \quad=N_{f} V T \sum_{n \in \mathbb{Z}} \int \frac{\mathrm{d}^{3} p}{(2 \pi)^{3}}\left(\partial_{k} r_{k}\right) \operatorname{tr}\left[(-i \vec{p})\left(\begin{array}{cc}
G^{+} & -G_{0}^{+} D^{-} G^{-} \\
-G_{0}^{-} D^{+} G^{+} & G^{-}
\end{array}\right)\right] \\
& \quad=N_{f} V T \sum_{n \in \mathbb{Z}} \int \frac{\mathrm{d}^{3} p}{(2 \pi)^{3}}\left(\partial_{k} r_{k}\right) \operatorname{tr}\left[(-i \vec{p})\left(G^{+}+G^{-}\right)\right] \\
& \quad=4 N_{f} V T \sum_{n \in \mathbb{Z}} \int \frac{\mathrm{d}^{3} p}{(2 \pi)^{3}} \vec{p}^{2}\left(\partial_{k} r_{k}\right)\left(1+r_{k}\right)\left[\frac{1-\frac{\mu}{E}}{p_{0}^{2}+\left(E_{p}^{-}\right)^{2}}+\frac{\left.1+\frac{\mu}{p_{0}^{2}+\left(E_{p}^{+}\right)^{2}}\right]}{1+\frac{\mu}{E_{k}}}\right] \\
& \quad=4 N_{f} \frac{4 \pi}{(2 \pi)^{3}} V T \sum_{n \in \mathbb{Z}} \int_{0}^{k} \mathrm{~d} p p^{2} k\left[\frac{1-\frac{\mu}{E_{k}}}{p_{0}^{2}+\left(E_{k}^{-}\right)^{2}}+\frac{1}{p_{0}^{2}+\left(E_{k}^{+}\right)^{2}}\right] \\
& \quad=4 N_{f} \frac{4 \pi}{3(2 \pi)^{3}} V T \sum_{n \in \mathbb{Z}} k^{4}\left[\frac{1-\frac{\mu}{E_{k}}}{p_{0}^{2}+\left(E_{k}^{-}\right)^{2}}+\frac{1+\frac{\mu}{E_{k}^{2}}}{p_{0}^{2}+\left(E_{k}^{+}\right)^{2}}\right] .
\end{aligned}
$$

In the penultimate step we inserted the Litim regulator, and replaced the energy functions by

$$
\begin{aligned}
E_{k} & =\sqrt{k^{2}+\left(h_{\phi} \sigma\right)^{2}}, \\
E_{k}^{ \pm} & =\sqrt{\left(E_{k} \pm \mu\right)^{2}+\left(h_{\Delta} d\right)^{2}} .
\end{aligned}
$$

We are left to evaluate the Matsubara sum. To this end, we replace $p_{0}$ with the fermionic Matsubara frequencies, $v_{n}=(2 n+1) \pi T$, which then yields the following result: 


$$
\begin{aligned}
& \operatorname{Tr}\left(\frac{-i \vec{p} \partial_{k} r_{k}(\vec{p})}{\Gamma_{k, \mathrm{rg}}^{(2)}-i \vec{p} r_{k}(\vec{p}) \otimes \mathbb{1}_{2 \times 2}}\right) \\
& \quad=4 N_{f} \frac{4 \pi}{3(2 \pi)^{3}} V T \sum_{n \in \mathbb{Z}} k^{4}\left[\frac{1-\frac{\mu}{E_{k}}}{v_{n}^{2}+\left(E_{k}^{-}\right)^{2}}+\frac{1+\frac{\mu}{E_{k}}}{v_{n}^{2}+\left(E_{k}^{+}\right)^{2}}\right] \\
& =4 N_{f} \frac{4 \pi k^{4}}{6(2 \pi)^{3}} V \sum_{ \pm} \frac{1 \pm \frac{\mu}{E_{k}}}{E_{k}^{ \pm}} \tanh \frac{E_{k}^{ \pm}}{2 T} \\
& \quad=\frac{2 k^{4}}{3 \pi^{2}} V \sum_{ \pm} \frac{1 \pm \frac{\mu}{E_{k}}}{E_{k}^{ \pm}} \tanh \frac{E_{k}^{ \pm}}{2 T} .
\end{aligned}
$$

Next, we evaluate the ungapped contribution, which except for a factor of $\frac{1}{3}$ yields the same result as for the quark-meson model. The inverse is found to be

$$
\left(\Gamma_{k, \mathrm{~b}}^{(2)}-i \vec{p} r_{k}(\vec{p})\right)^{-1}=\frac{i \vec{p}\left(1+r_{k}\right)+\gamma_{0}\left(\mu+i p_{0}\right)+h_{\phi} \sigma}{k^{2}+\left(p_{0}-i \mu\right)^{2}+\left(h_{\phi} \sigma\right)^{2}} .
$$

Next, the resulting contribution to the flow equation is obtained,

$$
\begin{aligned}
& \operatorname{Tr}\left(\frac{-i \vec{p} \partial_{k} r_{k}(\vec{p})}{\Gamma_{k, \mathrm{~b}}^{(2)}-i \vec{p} r_{k}(\vec{p})}\right) \\
& =N_{f} V T \sum_{n \in \mathbb{Z}} \int \frac{\mathrm{d}^{3} p}{(2 \pi)^{3}}\left(\partial_{k} r_{k}\right) \operatorname{tr}\left\{(-i \vec{p})\left[\frac{i \vec{p}\left(1+r_{k}\right)+\gamma_{0}\left(\mu+i p_{0}\right)+h_{\phi} \sigma}{k^{2}+\left(p_{0}-i \mu\right)^{2}+\left(h_{\phi} \sigma\right)^{2}}\right]\right\} \\
& =4 N_{f} V T \sum_{n \in \mathbb{Z}} \int \frac{\mathrm{d}^{3} p}{(2 \pi)^{3}} \frac{k}{k^{2}+\left(p_{0}-i \mu\right)^{2}+\left(h_{\phi} \sigma\right)^{2}} \\
& =4 N_{f} \frac{4 \pi}{(2 \pi)^{3}} V T \sum_{n \in \mathbb{Z}} \int_{0}^{k} \mathrm{~d} p \frac{p^{2} k}{k^{2}+\left(p_{0}-i \mu\right)^{2}+\left(h_{\phi} \sigma\right)^{2}} \\
& =4 N_{f} \frac{4 \pi}{3(2 \pi)^{3}} V T \sum_{n \in \mathbb{Z}} \frac{k^{4}}{k^{2}+\left(p_{0}-i \mu\right)^{2}+\left(h_{\phi} \sigma\right)^{2}} \\
& =N_{f} \frac{4 \pi}{3(2 \pi)^{3}} V \frac{k^{4}}{E_{k}}\left(\tanh \frac{E_{k}-\mu}{2 T}+\tanh \frac{E_{k}+\mu}{2 T}\right) \\
& =\frac{V}{3 \pi^{2}} \frac{k^{4}}{E_{k}}\left(\tanh \frac{E_{k}-\mu}{2 T}+\tanh \frac{E_{k}+\mu}{2 T}\right) .
\end{aligned}
$$

Finally, the total fermionic contribution to the flow equation reads: 
$\partial_{k} U_{k, \mathrm{~F}}=-\frac{k^{4}}{3 \pi^{2}}\left[2 \sum_{ \pm} \frac{1 \pm \frac{\mu}{E_{k}}}{E_{k}^{ \pm}} \tanh \frac{E_{k}^{ \pm}}{2 T}+\frac{1}{E_{k}}\left(\tanh \frac{E_{k}-\mu}{2 T}+\tanh \frac{E_{k}+\mu}{2 T}\right)\right]$

The complete flow equation containing both the bosonic and fermionic contributions is therefore obtained as follows:

$$
\begin{aligned}
\partial_{k} U_{k}= & \frac{k^{4}}{12 \pi^{2}}\left[\frac{3}{E_{\pi, k}} \operatorname{coth} \frac{E_{\pi, k}}{2 T}+\sum_{i=1}^{3} \frac{\alpha_{0}-\alpha_{1} z_{i}^{2}+3 z_{i}^{4}}{\left(z_{i+1}^{2}-z_{i}^{2}\right)\left(z_{i+2}^{2}-z_{i}^{2}\right) z_{i}} \operatorname{coth} \frac{z_{i}}{2 T}\right. \\
& \left.+\frac{2}{\tilde{E}_{\Delta, 0, k}}\left(\operatorname{coth} \frac{\tilde{E}_{\Delta, 0, k}-2 \mu}{2 T}+\operatorname{coth} \frac{\tilde{E}_{\Delta, 0, k}+2 \mu}{2 T}\right)\right] \\
& -\frac{k^{4}}{3 \pi^{2}}\left[2 \sum_{ \pm} \frac{1 \pm \frac{\mu}{E_{k}}}{E_{k}^{ \pm}} \tanh \frac{E_{k}^{ \pm}}{2 T}+\frac{1}{E_{k}}\left(\tanh \frac{E_{k}-\mu}{2 T}+\tanh \frac{E_{k}+\mu}{2 T}\right)\right] .
\end{aligned}
$$




\section{Appendix E}

\section{Flow of the Two-Point Function at Finite External Momenta}

Here we present the detailed calculation for the derivation of the flow equation for the two-point function at finite external momenta.

\section{E.1 Wetterich equation for the two-point function}

First, we would like to derive the proper variational derivative of a generic two-point function $\Gamma^{(2)}$ taking into account the explicit momentum dependence. To this end, we first note that the functional $\mathbb{1}$ is given as

$$
\int_{p} \Gamma^{(2)}(q, p) \Gamma^{(2)}(p, r)^{-1}=\delta(q-r)
$$

Now, let us take the functional derivative,

$$
\begin{aligned}
& \frac{\delta}{\delta \phi(s)} \delta(q-r)=0 \\
&=\int_{p}\left(\frac{\delta}{\delta \phi(s)} \Gamma^{(2)}(q, p)\right) \Gamma^{(2)}(p, r)^{-1}+\int_{p} \Gamma^{(2)}(q, p) \frac{\delta}{\delta \phi(s)} \Gamma^{(2)}(p, r)^{-1} \\
&=\int_{p} \Gamma^{(3)}(q, p, s) \Gamma^{(2)}(p, r)^{-1}+\int_{p} \Gamma^{(2)}(q, p) \frac{\delta}{\delta \phi(s)} \Gamma^{(2)}(p, r)^{-1} \\
& \Leftrightarrow \int_{p} \Gamma^{(2)}(q, p) \frac{\delta}{\delta \phi(s)} \Gamma^{(2)}(p, r)^{-1}=-\int_{p} \Gamma^{(3)}(q, p, s) \Gamma^{(2)}(p, r)^{-1}
\end{aligned}
$$

we multiply by $\Gamma^{(2)}(t, q)^{-1}$ from the left and integrate over $q$, 


$$
\begin{aligned}
& \int_{p, q} \underbrace{\Gamma^{(2)}(t, q)^{-1} \Gamma^{(2)}(q, p)}_{\delta(t-p)} \frac{\delta}{\delta \phi(s)} \Gamma^{(2)}(p, r)^{-1} \\
= & -\int_{p, q} \Gamma^{(2)}(t, q)^{-1} \Gamma^{(3)}(q, p, s) \Gamma^{(2)}(p, r)^{-1} \\
\Leftrightarrow & \frac{\delta}{\delta \phi(s)} \Gamma^{(2)}(t, r)^{-1}=-\int_{p, q} \Gamma^{(2)}(t, q)^{-1} \Gamma^{(3)}(q, p, s) \Gamma^{(2)}(p, r)^{-1} .
\end{aligned}
$$

Next, we can evaluate the second variational derivative,

$$
\begin{aligned}
& \frac{\delta}{\delta \phi(u)} \frac{\delta}{\delta \phi(s)} \Gamma^{(2)}(t, r)^{-1} \\
& =\int_{p_{1}, p_{2}} \int_{q_{1}, q_{2}} \Gamma^{(2)}\left(t, q_{1}\right)^{-1} \Gamma^{(3)}\left(q_{1}, q_{2}, u\right) \Gamma^{(2)}\left(q_{2}, p_{1}\right)^{-1} \Gamma^{(3)}\left(p_{1}, p_{2}, s\right) \Gamma^{(2)}\left(p_{2}, r\right)^{-1} \\
& \quad+\int_{p_{1}, p_{2}} \int_{q_{1}, q_{2}} \Gamma^{(2)}\left(t, p_{1}\right)^{-1} \Gamma^{(3)}\left(p_{1}, p_{2}, s\right) \Gamma^{(2)}\left(p_{2}, q_{1}\right)^{-1} \Gamma^{(3)}\left(q_{1}, q_{2}, u\right) \Gamma^{(2)}\left(q_{2}, r\right)^{-1} \\
& \quad-\int_{p_{1}, p_{2}} \Gamma^{(2)}\left(t, p_{1}\right)^{-1} \Gamma^{(4)}\left(p_{1}, p_{2}, s, u\right) \Gamma^{(2)}\left(p_{2}, r\right)^{-1}
\end{aligned}
$$

The n-point functions respect energy-momentum conservation, therefore we can write

$$
\Gamma^{(n)}\left(p_{1}, \ldots, p_{n}\right)=\Gamma^{(n)}\left(p_{1}, \ldots, p_{n-1}\right) \delta\left(p_{1}+\ldots+p_{n}\right) .
$$

Then, we apply this identity and integrate Eq. (E.4) over the momenta $t$ and $r$, which yields:

$$
\begin{aligned}
& \frac{\delta}{\delta \phi(u)} \frac{\delta}{\delta \phi(s)} \int_{t, r} \Gamma^{(2)}(t, r)^{-1}=\frac{\delta}{\delta \phi(u)} \frac{\delta}{\delta \phi(s)} \int_{t} \Gamma^{(2)}(t)^{-1} \\
& =\int_{p_{1}, p_{2}, q_{1}, q_{2}, t, r} \Gamma^{(2)}\left(t, q_{1}\right)^{-1} \Gamma^{(3)}\left(q_{1}, q_{2}, u\right) \Gamma^{(2)}\left(q_{2}, p_{1}\right)^{-1} \Gamma^{(3)}\left(p_{1}, p_{2}, s\right) \Gamma^{(2)}\left(p_{2}, r\right)^{-1} \\
& +\int_{p_{1}, p_{2}, q_{1}, q_{2}, t, r} \Gamma^{(2)}\left(t, p_{1}\right)^{-1} \Gamma^{(3)}\left(p_{1}, p_{2}, s\right) \Gamma^{(2)}\left(p_{2}, q_{1}\right)^{-1} \Gamma^{(3)}\left(q_{1}, q_{2}, u\right) \Gamma^{(2)}\left(q_{2}, r\right)^{-1} \\
& -\int_{p_{1}, p_{2}, t, r} \Gamma^{(2)}\left(t, p_{1}\right)^{-1} \Gamma^{(4)}\left(p_{1}, p_{2}, s, u\right) \Gamma^{(2)}\left(p_{2}, r\right)^{-1} \\
& =\int_{r} \Gamma^{(2)}(r)^{-1} \Gamma^{(3)} \Gamma^{(2)}(r-u)^{-1} \Gamma^{(3)} \Gamma^{(2)}(r)^{-1} \\
& \quad+\int_{r} \Gamma^{(2)}(r)^{-1} \Gamma^{(3)} \Gamma^{(2)}(r-s)^{-1} \Gamma^{(3)} \Gamma^{(2)}(r)^{-1} \\
& \quad-\int_{r} \Gamma^{(2)}(r)^{-1} \Gamma^{(4)} \Gamma^{(2)}(r)^{-1}
\end{aligned}
$$

Here we have dropped the momentum dependence of the three- and fourpoint functions as they do not depend on the momentum in our truncation. 
Setting $u=-s=p$, relabeling $r \rightarrow q$, and shifting the first line by $q \rightarrow q+p$ and second line by $q \rightarrow q-p$ we obtain

$$
\begin{aligned}
& \frac{\delta}{\delta \phi(p)} \frac{\delta}{\delta \phi(-p)} \int_{t} \Gamma^{(2)}(t)^{-1} \\
& =\int_{q} \Gamma^{(2)}(q+p)^{-1} \Gamma^{(3)} \Gamma^{(2)}(q)^{-1} \Gamma^{(3)} \Gamma^{(2)}(q+p)^{-1} \\
& +\int_{q} \Gamma^{(2)}(q-p)^{-1} \Gamma^{(3)} \Gamma^{(2)}(q)^{-1} \Gamma^{(3)} \Gamma^{(2)}(q-p)^{-1} \\
& -\int_{q} \Gamma^{(2)}(q)^{-1} \Gamma^{(4)} \Gamma^{(2)}(q)^{-1}
\end{aligned}
$$

Finally, we can insert this result into the Wetterich equation, which yields:

$$
\begin{aligned}
\partial_{k} \Gamma_{k}^{(2)}(p)= & \frac{1}{2} S \operatorname{Tr}\left[\partial_{k} R_{k}(q+p) G_{k}(q+p) \Gamma_{k}^{(3)} G_{k}(q) \Gamma_{k}^{(3)} G_{k}(q+p)\right] \\
& +\frac{1}{2} S \operatorname{Tr}\left[\partial_{k} R_{k}(q-p) G_{k}(q-p) \Gamma_{k}^{(3)} G_{k}(q) \Gamma_{k}^{(3)} G_{k}(q-p)\right] \\
& -\frac{1}{2} S \operatorname{STr}\left[\partial_{k} R_{k}(q) G_{k}(q) \Gamma_{k}^{(4)} G_{k}(q)\right] .
\end{aligned}
$$

Here we defined $G_{k} \equiv\left(\Gamma_{k}^{(2)}+R_{k}\right)^{-1}$. This expression is valid for both bosonic and fermionic two-point functions. In the next step we show, that the first and the second line of the above expression are actually the same. For the bosonic case this is rather easy to show

$$
\begin{aligned}
& \int_{\vec{q}} \partial_{k} R_{k}\left((\vec{q}+\vec{p})^{2}\right) G_{k}\left((\vec{q}+\vec{p})^{2}\right) G_{k}\left(\vec{q}^{2}\right) G_{k}\left((\vec{q}+\vec{p})^{2}\right) \\
= & \int_{-\infty}^{\infty} \mathrm{d} q_{x} \mathrm{~d} q_{y} \mathrm{~d} q_{z} \partial_{k} R_{k}\left(\vec{q}^{2}+\vec{p}^{2}+2 \vec{q} \cdot \vec{p}\right) G_{k}\left(\vec{q}^{2}+\vec{p}^{2}+2 \vec{q} \cdot \vec{p}\right) G_{k}\left(\vec{q}^{2}\right) G_{k}\left(\vec{q}^{2}+\vec{p}^{2}+2 \vec{q} \cdot \vec{p}\right) \\
\stackrel{\vec{q} \rightarrow-\vec{q}}{=}-\int_{\infty}^{-\infty} \mathrm{d} q_{x} \mathrm{~d} q_{y} \mathrm{~d} q_{z} \partial_{k} R_{k}\left(\vec{q}^{2}+\vec{p}^{2}-2 \vec{q} \cdot \vec{p}\right) G_{k}\left(\vec{q}^{2}+\vec{p}^{2}-2 \vec{q} \cdot \vec{p}\right) G_{k}\left(\vec{q}^{2}\right) G_{k}\left(\vec{q}^{2}+\vec{p}^{2}-2 \vec{q} \cdot \vec{p}\right) & \\
= & \int_{-\infty}^{\infty} \mathrm{d} q_{x} \mathrm{~d} q_{y} \mathrm{~d} q_{z} \partial_{k} R_{k}\left(\vec{q}^{2}+\vec{p}^{2}-2 \vec{q} \cdot \vec{p}\right) G_{k}\left(\vec{q}^{2}+\vec{p}^{2}-2 \vec{q} \cdot \vec{p}\right) G_{k}\left(\vec{q}^{2}\right) G_{k}\left(\vec{q}^{2}+\vec{p}^{2}-2 \vec{q} \cdot \vec{p}\right) \\
= & \int_{\vec{q}} \partial_{k} R_{k}\left((\vec{q}-\vec{p})^{2}\right) G_{k}\left((\vec{q}-\vec{p})^{2}\right) G_{k}\left(\vec{q}^{2}\right) G_{k}\left((\vec{q}-\vec{p})^{2}\right) .
\end{aligned}
$$

We have not considered the zeroth components of the momentum four vectors, because in this work we set $p_{0}=0$. For the fermionic contribution we first perform the trace, such that odd numbers of Dirac slashed quantities vanish. It then turns out that the momentum dependencies are either of the form $\vec{q}^{2}$ or $\cos \varphi=\frac{\vec{q} \cdot(\vec{q} \pm \vec{p})}{|\vec{q}||\vec{q} \pm \vec{p}|}$ such that the above calculation holds for fermions as well.

Thus, we can simplify Eq. (E.8) to 


$$
\begin{aligned}
\partial_{k} \Gamma_{k}^{(2)}(p)= & \operatorname{STr}\left[\partial_{k} R_{k}(q+p) G_{k}(q+p) \Gamma_{k}^{(3)} G_{k}(q) \Gamma_{k}^{(3)} G_{k}(q+p)\right] \\
& -\frac{1}{2} S \operatorname{Tr}\left[\partial_{k} R_{k}(q) G_{k}(q) \Gamma_{k}^{(4)} G_{k}(q)\right]
\end{aligned}
$$

\section{E.2 Simplification of the Two-Point Function for Finite External Momentum}

At finite external momentum, the first term in the flow equation (E.10) contains two different Heaviside functions when using the Litim regulator. In the numerator of $\partial_{k} R_{k}$ and the denominator of $G_{k}(q+p)$ we have the Heaviside function $\theta\left(k^{2}-(\vec{q}+\vec{p})^{2}\right)$. If this Heaviside step function vanishes, the total integral vanishes. On the other hand, we have also another Heaviside function contained in a part of the denominator of $G_{k}(q)$, namely $\theta\left(k^{2}-\vec{q}^{2}\right)$. Depending on whether this Heaviside function is zero or not, we have two different expressions. Thus, we can write

$$
\int \mathrm{d}^{3} q \theta\left(k^{2}-(\vec{q}+\vec{p})^{2}\right)\left[\theta\left(k^{2}-\vec{q}^{2}\right) f_{1}+\theta\left(-k^{2}+\vec{q}^{2}\right) f_{2}\right]
$$

where $f_{1}$ and $f_{2}$ are the resulting expressions of $G_{k}(q+p) \Gamma_{k}^{(3)} G_{k}(q) \Gamma_{k}^{(3)} G_{k}(q+$ $p$ ) for $k^{2}>\vec{q}^{2}$ and for $k^{2}<\vec{q}^{2}$, respectively. In the following, we will use spherical coordinates and choose the reference frame such that the external momentum vector points in the z-direction, $\vec{p}^{\mathrm{T}}=(0,0, p)$ :

$$
\begin{aligned}
& 2 \pi \int_{0}^{\pi} \mathrm{d} \theta \sin \theta \int_{0}^{\infty} \mathrm{d} q q^{2} \theta\left(k^{2}-q^{2}-p^{2}-2 q p \cos \theta\right)\left[\theta\left(k^{2}-q^{2}\right) f_{1}+\theta\left(-k^{2}+q^{2}\right) f_{2}\right] \\
& =2 \pi \int_{0}^{2} \mathrm{~d} v \int_{0}^{\infty} \mathrm{d} q q^{2} \theta\left(k^{2}-q^{2}-p^{2}+2 q p-2 v q p\right)\left[\theta\left(k^{2}-q^{2}\right) f_{1}+\theta\left(-k^{2}+q^{2}\right) f_{2}\right] \\
& =2 \pi \int_{0}^{2} \mathrm{~d} v \int_{0}^{\infty} \mathrm{d} q q^{2} \theta\left(\frac{k^{2}-(q-p)^{2}}{2 q p}-v\right)\left[\theta\left(k^{2}-q^{2}\right) f_{1}+\theta\left(-k^{2}+q^{2}\right) f_{2}\right] \\
& =2 \pi \int_{0}^{\infty} \mathrm{d} q q^{2}\left[\int_{0}^{2} \mathrm{~d} v \theta\left(\frac{k^{2}-(q-p)^{2}}{2 q p}-2\right)\right.
\end{aligned}
$$

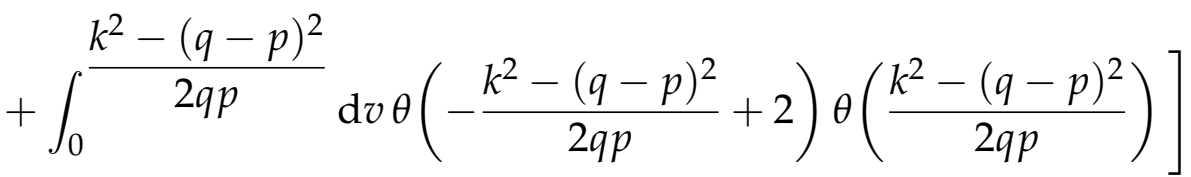

$$
\begin{aligned}
& \times\left[\theta\left(k^{2}-q^{2}\right) f_{1}+\theta\left(-k^{2}+q^{2}\right) f_{2}\right] .
\end{aligned}
$$

In the second line we substituted $v \equiv \cos \theta+1$. We have distinguished between three cases: $\frac{k^{2}-(q-p)^{2}}{2 q p}>2 \geq v, 0<\frac{k^{2}-(q-p)^{2}}{2 q p}<2$, and 
$0>\frac{k^{2}-(q-p)^{2}}{2 q p}$. In the first case, the $v$ integration does not depend on $q$. In the last case, the integral vanishes automatically. Let us now consider all possible combinations and simplify as much as possible:

$$
\begin{aligned}
\text { (1) } & =2 \pi \int_{0}^{\infty} \mathrm{d} q q^{2} \theta\left(\frac{k^{2}-(q-p)^{2}}{2 q p}-2\right) \theta\left(k^{2}-q^{2}\right) \int_{0}^{2} \mathrm{~d} v f_{1} \\
& =2 \pi \int_{0}^{\infty} \mathrm{d} q q^{2} \theta\left(k^{2}-(q+p)^{2}\right) \int_{0}^{2} \mathrm{~d} v f_{1}=2 \pi \int_{0}^{k-p} \mathrm{~d} q q^{2} \theta(k-p) \int_{0}^{2} \mathrm{~d} v f_{1}, \\
\text { (E.13) } & \\
\text { (2) } & =2 \pi \int_{0}^{\infty} \mathrm{d} q q^{2} \theta\left(\frac{k^{2}-(q-p)^{2}}{2 q p}-2\right) \theta\left(-k^{2}+q^{2}\right) \int_{0}^{2} \mathrm{~d} v f_{2}=0, \quad \text { (E.14) } \\
\text { (3) } & =2 \pi \int_{0}^{\infty} \mathrm{d} q q^{2} \theta\left(-\frac{k^{2}-(q-p)^{2}}{2 q p}+2\right) \theta\left(\frac{k^{2}-(q-p)^{2}}{2 q p}\right) \theta\left(k^{2}-q^{2}\right) \int_{0}^{v_{\max }} \mathrm{d} v f_{1}, \\
& =2 \pi\left[\int_{p-k}^{k} \mathrm{~d} q q^{2} \theta\left(k-\frac{p}{2}\right) \theta(p-k) \int_{0}^{v_{\max }} \mathrm{d} v f_{1}+\int_{k-p}^{k} \mathrm{~d} q q^{2} \theta(k-p) \int_{0}^{v_{\max }} \mathrm{d} v f_{1}\right],
\end{aligned}
$$

$$
\text { (4) } \begin{aligned}
& =2 \pi \int_{0}^{\infty} \mathrm{d} q q^{2} \theta\left(-\frac{k^{2}-(q-p)^{2}}{2 q p}+2\right) \theta\left(\frac{k^{2}-(q-p)^{2}}{2 q p}\right) \theta\left(-k^{2}+q^{2}\right) \int_{0}^{v_{\max }} \mathrm{d} v f_{2} \\
& =2 \pi\left[\int_{p-k}^{k+p} \mathrm{~d} q q^{2} \theta\left(\frac{p}{2}-k\right) \int_{0}^{v_{\max }} \mathrm{d} v f_{2}+\int_{k}^{k+p} \mathrm{~d} q q^{2} \theta\left(k-\frac{p}{2}\right) \int_{0}^{v_{\max }} \mathrm{d} v f_{2}\right] .
\end{aligned}
$$

The complete result is then simply the sum of these integrals, (1) + (2) + (3) + (4). Furthermore, we have defined $v_{\max }=\frac{k^{2}-(q-p)^{2}}{2 q p}$. In this thesis, we perform first the traces on the several spaces, then perform the Matsubara sum, and lastly carry out the integration using Eqs. (E.13) to (E.16) last.

\section{E.3 Flow of the Two-Point Function for the Quark- Meson-Model}

Here we first derive the flow of the pion two-point function for the quarkmeson-model to showcase the general structure of the derivation. For the quark-meson-diquark model the calculation can be done analogously as we will see in the next section. The effective action for the quark-meson model is given below, 


$$
\begin{aligned}
\Gamma_{\mathrm{QM}}[\Phi]=\int \mathrm{d}^{4} x\left\{\bar{\psi}\left[\not \partial-\mu \gamma_{0}+h_{\phi}\left(\sigma+i \gamma_{5} \vec{\tau} \cdot \vec{\pi}\right)\right] \psi\right. \\
\left.+\frac{1}{2}\left[\left(\partial_{\mu} \sigma\right)^{2}+\left(\partial_{\mu} \vec{\pi}\right)^{2}\right]+U_{k}\left(\phi^{2}, \Delta^{2}\right)-c \sigma\right\} .
\end{aligned}
$$

Next, we can use Eq. (E.10) for the pionic two-point function:

$$
\begin{aligned}
\partial_{k} \Gamma_{k, \pi}^{(2)}(p)= & \partial_{k} \Gamma_{k, \pi}^{(2), \mathrm{B}}(p)+\partial_{k} \Gamma_{k, \pi}^{(2), \mathrm{F}}(p), \\
\partial_{k} \Gamma_{k, \pi}^{(2), \mathrm{B}}(p)= & \operatorname{Tr}\left[\partial_{k} R_{k}(q+p) G_{k, \mathrm{~B}}(q+p) \Gamma_{k, \pi}^{(3)} G_{k, \mathrm{~B}}(q) \Gamma_{k, \pi}^{(3)} G_{k, \mathrm{~B}}(q+p)\right] \\
& -\frac{1}{2} \operatorname{Tr}\left[\partial_{k} R_{k}(q) G_{k, \mathrm{~B}}(q) \Gamma_{k, \pi \pi}^{(4)} G_{k, \mathrm{~B}}(q)\right], \\
\partial_{k} \Gamma_{k, \pi}^{(2), \mathrm{F}}(p)= & -2 \operatorname{Tr}\left[\partial_{k} R_{k}(q+p) G_{k, \psi}(q+p) \Gamma_{k, \bar{\psi} \psi \pi}^{(2,1)} G_{k, \psi}(q) \Gamma_{k, \bar{\psi} \psi \pi}^{(2,1)} G_{k, \psi}(q+p)\right],
\end{aligned}
$$

where

$$
\begin{aligned}
& \left.G_{k, \mathrm{~B}} \equiv\left(\Gamma_{k, \mathrm{~B}}^{(2)}+R_{k}^{\mathrm{B}}\right)^{-1}\right|_{\vec{\pi}=0, \sigma=\sigma_{0}}=\left(\begin{array}{cc}
G_{k, \pi \pi \mathbb{1}_{3 \times 3}} & 0 \\
0 & G_{k, \sigma \sigma}
\end{array}\right), \\
& \left.G_{k, \alpha \alpha} \equiv\left(\Gamma_{k, \alpha \alpha}^{(2)}+R_{k}^{\mathrm{B}}\right)^{-1}\right|_{\vec{\pi}=0, \sigma=\sigma_{0}}{ }^{\prime} \\
& \Gamma_{k, \mathrm{~B}}^{(2)}=\left(\begin{array}{llll}
\Gamma_{k, \pi_{1} \pi_{1}}^{(2)} & \Gamma_{k, \pi_{1} \pi_{2}}^{(2)} & \Gamma_{k, \pi_{1} \pi_{3}}^{(2)} & \Gamma_{k, \pi_{1} \sigma}^{(2)} \\
\Gamma_{k, \pi_{2} \pi_{1}}^{(2)} & \Gamma_{k, \pi_{2} \pi_{2}}^{(2)} & \Gamma_{k, \pi_{2} \pi_{3}}^{(2)} & \Gamma_{k, \pi_{2} \sigma}^{(2)} \\
\Gamma_{k, \pi_{3} \pi_{1}}^{(2)} & \Gamma_{k, \pi_{3} \pi_{2}}^{(2)} & \Gamma_{k, \pi_{3} \pi_{3}}^{(2)} & \Gamma_{k, \pi_{3} \sigma}^{(2)} \\
\Gamma_{k, \sigma \pi_{1}}^{(2)} & \Gamma_{k, \sigma \pi_{2}}^{(2)} & \Gamma_{k, \sigma \pi_{3}}^{(2)} & \Gamma_{k, \sigma \sigma}^{(2)}
\end{array}\right), \\
& \Gamma_{k, \pi}^{(3)}=\left.\left(\frac{\delta}{\delta \pi} \Gamma_{k, \mathrm{~B}}^{(2)}\right)\right|_{\vec{\pi}=0, \sigma=\sigma_{0}}, \quad \Gamma_{k, \pi \pi}^{(4)}=\left.\left(\frac{\delta}{\delta \pi} \frac{\delta}{\delta \pi} \Gamma_{k, \mathrm{~B}}^{(2)}\right)\right|_{\vec{\pi}=0, \sigma=\sigma_{0}},
\end{aligned}
$$

where $\alpha, \beta \in\{\sigma, \pi\}$. The vertex functions are found to be

$$
\begin{aligned}
\Gamma_{k, \bar{\psi} \psi i}^{(2,1)} & =h_{\phi}\left\{\begin{array}{ll}
1 & i=0 \\
i \gamma_{5} \tau_{i} & i=1,2,3
\end{array},\right. \\
\Gamma_{k, \bar{\psi} \psi \phi_{i} \phi_{j}}^{(2,2)} & =0 .
\end{aligned}
$$

Note that when we write $\Gamma_{k, \pi}^{(2)}(p)$ we actually choose one pion component, e.g., $\pi \equiv \pi_{1}$. Inserting the above relations we can make the two-point function more explicit: 


$$
\begin{aligned}
& \partial_{k} \Gamma_{k, \pi}^{(2), \mathrm{B}}=\left(J_{k, \sigma \pi}(p)+J_{k, \pi \sigma}(p)\right)\left(\Gamma_{k, \sigma \pi \pi}^{(3)}\right)^{2}-\frac{1}{2}\left(I_{k, \sigma}^{(2)} \Gamma_{k, \sigma \sigma \pi \pi}^{(4)}+5 I_{k, \pi}^{(2)} \Gamma_{k, \pi \pi \tilde{\pi} \tilde{\pi})}^{(4)},\right. \\
& \partial_{k} \Gamma_{k, \pi}^{(2), \mathrm{F}}=-2 N_{c} N_{f} J_{k, \bar{\psi} \psi}^{(\pi)}(p),
\end{aligned}
$$

where the threshold functions $I_{k, \alpha}^{(2)}$ are defined as

$$
I_{k, \alpha}^{(2)} \equiv \operatorname{Tr}_{q}\left[\partial_{k} R_{k}^{\mathrm{B}}(q) G_{k, \alpha}^{2}(q)\right], \alpha \in\{\sigma, \pi\},
$$

The trace of field space, including flavor, color and Dirac space for the fermionic case, is already evaluated in Eqs. (E.25) and (E.26). Therefore, $\operatorname{Tr}_{q}$ denotes that the remaining momentum integration as well as the Matsubara summation still have to be performed. The loop functions are defined as

$$
\begin{aligned}
& J_{k, \alpha \beta}(p)=\operatorname{Tr}_{q}\left[\partial_{k} R_{k}^{\mathrm{B}}(q+p) G_{k, \alpha}(q+p)^{2} G_{k, \beta}(q)\right] \\
& J_{k, \bar{\psi} \psi}^{(\alpha)}(p)=\operatorname{Tr}_{q}\left[\partial_{k} R_{k}^{\mathrm{F}}(q+p) G_{k, \psi}(q+p) \Gamma_{\bar{\psi} \psi \alpha}^{(2,1)} G_{k, \psi}(q) \Gamma_{\bar{\psi} \psi \alpha}^{(2,1)} G_{k, \psi}(q+p)\right] .
\end{aligned}
$$

The mesonic vertex functions derived from the effective potential $U_{k}\left(\phi^{2}\right)$ are found to be

$$
\begin{aligned}
\Gamma_{k, \phi_{i} \phi_{j} \phi_{m}}^{(3)} & =4 U_{k}^{(2)}\left(\delta_{i j} \phi_{m}+\delta_{i m} \phi_{j}+\delta_{j m} \phi_{i}\right)+8 U_{k}^{(3)} \phi_{i} \phi_{j} \phi_{m} \\
\Gamma_{k, \phi_{i} \phi_{j} \phi_{m} \phi_{n}}^{(4)} & =4 U_{k}^{(2)}\left(\delta_{i j} \delta_{m n}+\delta_{i n} \delta_{j m}+\delta_{j n} \delta_{i m}\right) \\
& +8 U_{k}^{(3)}\left(\delta_{i j} \phi_{m} \phi_{n}+\delta_{j m} \phi_{i} \phi_{n}+\delta_{m n} \phi_{i} \phi_{j}+\delta_{j n} \phi_{i} \phi_{m}+\delta_{i n} \phi_{j} \phi_{m}+\delta_{i m} \phi_{j} \phi_{n}\right) \\
& +16 U_{k}^{(4)} \phi_{i} \phi_{j} \phi_{m} \phi_{n} .
\end{aligned}
$$

Inserting all definitions yields the loop functions

$$
J_{k, \alpha \beta}=\operatorname{Tr}_{q}\left[\frac{2 k \theta\left(k^{2}-\left(\vec{q}^{2}+\vec{p}^{2}\right)\right)}{\left(E_{\alpha, k}^{2}+q_{0}^{2}\right)^{2}\left[m_{\beta}^{2}+q_{0}^{2}+\vec{q}^{2}+\left(k^{2}-\vec{q}^{2}\right) \theta\left(k^{2}-\vec{q}^{2}\right)\right]}\right]
$$




$$
\begin{aligned}
& J_{k, \bar{\psi} \psi}^{(\pi)}= \\
& 4 h_{\phi}^{2} \operatorname{Tr}_{q} \theta\left(k^{2}-\left(\vec{q}^{2}+\vec{p}^{2}\right)\right)\{ \\
& \times \frac{\left[1+\left(\frac{k}{|\vec{q}|}-1\right) \theta\left(k^{2}-\vec{q}^{2}\right)\right]|\vec{q}| \cos \varphi\left[-k^{2}+\left(q_{0}+p_{0}-i \mu\right)^{2}+\left(h_{\phi} \sigma\right)^{2}\right]}{\left[E_{k}^{2}+\left(q_{0}+p_{0}-i \mu\right)^{2}\right]^{2}\left(\left(h_{\phi} \sigma\right)^{2}+\left(q_{0}-i \mu\right)^{2}+\vec{q}^{2}\left[1+\left(\frac{k}{|\vec{q}|}-1\right) \theta\left(k^{2}-\vec{q}^{2}\right)\right]^{2}\right)} \\
& \left.+\frac{k\left[-2\left(h_{\phi} \sigma\right)^{2}-2\left(q_{0}-i \mu\right)\left(q_{0}+p_{0}-i \mu\right)\right]}{\left[E_{k}^{2}+\left(q_{0}+p_{0}-i \mu\right)^{2}\right]^{2}\left(\left(h_{\phi} \sigma\right)^{2}+\left(q_{0}-i \mu\right)^{2}+\vec{q}^{2}\left[1+\left(\frac{k}{|\vec{q}|}-1\right) \theta\left(k^{2}-\vec{q}^{2}\right)\right]^{2}\right)}\right\} .
\end{aligned}
$$

where $\cos \varphi=\frac{\vec{q} \cdot(\vec{q}+\vec{p})}{|\vec{q}||\vec{q}+\vec{p}|}$. Note, that we have set $\theta\left(k^{2}-\left(\vec{q}^{2}+\vec{p}^{2}\right)\right) \rightarrow 1$ in the denominators since for the case where they vanish, the full integrand vanishes as well. Depending on the value of the Heaviside function $\theta\left(k^{2}-\vec{q}^{2}\right)$, we have two different expressions for the loop function, namely one where $\theta\left(k^{2}-\vec{q}^{2}\right)=0$ and one where $\theta\left(k^{2}-\vec{q}^{2}\right)=1$. In the following, we need to perform the Matsubara sums for each of the two cases, which can be done analytically even though the resulting expressions are quite lengthy. Ultimately, we can perform the integration using Eqs. (E.13) to (E.16), where $f_{1}$ and $f_{2}$ are the Matsubara sums of the loop functions for $k^{2}>\vec{q}^{2}$ and $k^{2}<\vec{q}^{2}$, respectively. Here it is possible to perform the angular integration analytically for all loop functions, while the integration over the inner momenta has to be done numerically. As an example, we will perform this calculation for the bosonic loop function more explicitly by using (E.13) to (E.16): 


$$
\begin{aligned}
J_{k, \alpha \beta} & =\int \mathrm{d}^{3} q \theta\left(k^{2}-(\vec{q}+\vec{p})^{2}\right)\left[\theta\left(k^{2}-\vec{q}^{2}\right) J_{k, \alpha \beta}^{(1)}+\theta\left(-k^{2}+\vec{q}^{2}\right) J_{k, \alpha \beta}^{(2)}\right] \\
= & 2 \pi\left[\int_{0}^{k-p} \mathrm{~d} q q^{2} \theta(k-p) \int_{0}^{2} \mathrm{~d} v J_{k, \alpha \beta}^{(1)}+\int_{p-k}^{k} \mathrm{~d} q q^{2} \theta\left(k-\frac{p}{2}\right) \theta(p-k) \int_{0}^{v_{\max }} \mathrm{d} v J_{k, \alpha \beta}^{(1)}\right. \\
& +\int_{k-p}^{k} \mathrm{~d} q q^{2} \theta(k-p) \int_{0}^{v_{\max }} \mathrm{d} v J_{k, \alpha \beta}^{(1)}+\int_{p-k}^{k+p} \mathrm{~d} q q^{2} \theta\left(\frac{p}{2}-k\right) \int_{0}^{v_{\max }} \mathrm{d} v J_{k, \alpha \beta}^{(2)} \\
& \left.+\int_{k}^{k+p} \mathrm{~d} q q^{2} \theta\left(k-\frac{p}{2}\right) \int_{0}^{v_{\max }} \mathrm{d} v J_{k, \alpha \beta}^{(2)}\right] \\
= & 2 \pi\left[\int_{0}^{k-p} \mathrm{~d} q q^{2} \theta(k-p) 2 J_{k, \alpha \beta}^{(1)}+\int_{p-k}^{k} \mathrm{~d} q q^{2} \theta\left(k-\frac{p}{2}\right) \theta(p-k) v_{\max } J_{k, \alpha \beta}^{(1)}\right. \\
& +\int_{k-p}^{k} \mathrm{~d} q q^{2} \theta(k-p) v_{\max } J_{k, \alpha \beta}^{(1)}+\int_{p-k}^{k+p} \mathrm{~d} q q^{2} \theta\left(\frac{p}{2}-k\right) v_{\max } J_{k, \alpha \beta}^{(2)} \\
& \left.+\int_{k}^{k+p} \mathrm{~d} q q^{2} \theta\left(k-\frac{p}{2}\right) v_{\max } J_{k, \alpha \beta}^{(2)}\right]
\end{aligned}
$$

where

$$
\begin{aligned}
& J_{k, \alpha \beta}^{(1)}=T \sum_{n \in \mathbb{Z}} \frac{2 k}{\left(E_{\alpha, k}^{2}+\omega_{n}^{2}\right)^{2}\left(E_{\beta, k}^{2}+\omega_{n}^{2}\right)} \\
& J_{k, \alpha \beta}^{(2)}=T \sum_{n \in \mathbb{Z}} \frac{2 k}{\left(E_{\alpha, k}^{2}+\omega_{n}^{2}\right)^{2}\left(m_{\beta}^{2}+\omega_{n}^{2}+\vec{q}^{2}\right)} .
\end{aligned}
$$

Note that $J_{k, \alpha \beta}^{(1)}$ does not depend on the inner momentum $q$ because of the choice of the optimized Litim regulator. On the other hand, $J_{k, \alpha \beta}^{(2)}$ retains the momentum dependence because the Heaviside function $\theta\left(k^{2}-\vec{q}^{2}\right)$ vanishes for $|\vec{q}|>k$. We can therefore perform the momentum integrals containing $J_{k, \alpha \beta}^{(1)}$ in Eq. (E.34) analytically. This is not possible for $J_{k, \alpha \beta}^{(2)}$ because after evaluating the Matsubara sum the explicit $q$-dependence gets incorporated in the bosonic distribution function that emerges from the sum. We have also tried to integrate the summand of the Matsubara sum first, but this then leads to a more complicated summand, for which the Matsubara sum cannot be performed analytically anymore. Thus, the best way to proceed from here is to evaluate the remaining momentum integrals numerically.

For the case of the fermionic loop function $J_{k, \bar{\psi} \psi}^{(\pi)}$ all integrals need to be performed numerically after evaluating the Matsubara sum. 


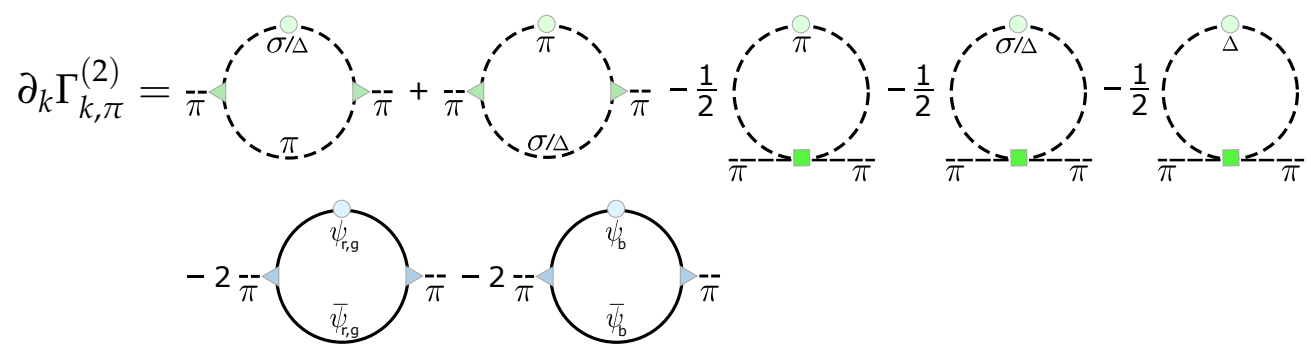

FIGURE E.1: Diagrammatic representation of the flow equation for the two-point function.

\section{E.4 Flow of the Two-Point Function for the Quark-Meson-Diquark-Model}

The calculations for the quark-meson diquark model are much more involved, therefore it is useful to use a high-level symbolic computation language, such as Mathematica. In App. F.4 a Mathematica script can be found, which contains a documented code for the calculations of the quark-meson-diquark model.

The difference to the previous calculation for the quark-meson model is that we have more diagrams to consider. A diagrammatic representation of the flow of the two-point function is found in Fig. E.1.

In the following, we sketch the derivation of the two-point function in the QMD model. The general structure is again given as

$$
\begin{aligned}
\partial_{k} \Gamma_{k, \pi}^{(2)}(p)= & \partial_{k} \Gamma_{k, \pi}^{(2), \mathrm{B}}(p)+\partial_{k} \Gamma_{k, \pi}^{(2), \mathrm{F}}(p), \\
\partial_{k} \Gamma_{k, \pi}^{(2), \mathrm{B}}(p)= & \operatorname{Tr}\left[\partial_{k} R_{k}(q+p) G_{k, \mathrm{~B}}(q+p) \Gamma_{k, \pi}^{(3)} G_{k, \mathrm{~B}}(q) \Gamma_{k, \pi}^{(3)} G_{k, \mathrm{~B}}(q+p)\right] \\
& -\frac{1}{2} \operatorname{Tr}\left[G_{k, \mathrm{~B}}(q) \Gamma_{k, \pi \pi}^{(4)} G_{k, \mathrm{~B}}(q)\right], \\
\partial_{k} \Gamma_{k, \pi}^{(2), \mathrm{F}}(p)= & -2 \operatorname{Tr}\left[\partial_{k} R_{k}(q+p) G_{k, \psi}(q+p) \Gamma_{k, \bar{\psi} \psi \pi}^{(2,1)} G_{k, \psi}(q) \Gamma_{k, \bar{\psi} \psi \pi}^{(2,1)} G_{k, \psi}(q+p)\right] .
\end{aligned}
$$

In the case of the QMD model the bosonic propagators are defined by

$$
\begin{aligned}
G_{k, \mathrm{~B}} & =\left(\begin{array}{ccc}
G_{k, \pi} \mathbb{1}_{3 \times 3} & 0 & 0 \\
0 & G_{k, \sigma-\Delta} & 0 \\
0 & 0 & G_{k, \Delta}
\end{array}\right), \\
G_{k, \alpha} & \equiv\left(\Gamma_{k, \alpha}^{(2)}+R_{k}^{\mathrm{B}}\right)^{-1},
\end{aligned}
$$

where $G_{k, \pi}, G_{k, \sigma-\Delta}$, and $G_{k, \Delta}$ are given by the expressions in Eqs. (D.17)(D.19). The three- and four-point functions are obtained by

$$
\Gamma_{k, \pi}^{(3)}=\left.\left(\frac{\delta}{\delta \pi} \Gamma_{k, \mathrm{~B}}^{(2)}\right)\right|_{\vec{\pi}=0, \sigma=\sigma_{0}}{ }^{\prime} \quad \Gamma_{k, \pi \pi}^{(4)}=\left.\left(\frac{\delta}{\delta \pi} \frac{\delta}{\delta \pi} \Gamma_{k, \mathrm{~B}}^{(2)}\right)\right|_{\vec{\pi}=0, \sigma=\sigma_{0}}{ }^{\prime}
$$


where $\Gamma_{k, \mathrm{~B}}^{(2)}$ in field space is defined by Eq. (D.4). Doing the explicit calculation for the three-point shows that almost all entries vanish except for

$$
\begin{gathered}
\Gamma_{k, \sigma \pi \pi}^{(3)}=\Gamma_{k, \pi \sigma \pi}^{(3)}=4 \sigma U_{k, \sigma \sigma}, \\
\Gamma_{k, \Delta \Delta_{2}^{\mathrm{R}} \pi \pi}^{(3)}=\Gamma_{k, \pi \Delta_{2}^{\mathrm{R}} \pi}^{(3)}=4 \Delta U_{k, \sigma \Delta} .
\end{gathered}
$$

And for the four-point function we have

$$
\begin{aligned}
& \Gamma_{k, \pi \pi}^{(4)}=\left(\begin{array}{ccc}
\Gamma_{k, \pi}^{(4)} & 0 & 0 \\
0 & \Gamma_{k, \sigma-\Delta}^{(4)} & 0 \\
0 & 0 & \Gamma_{k, \Delta}^{(4)}
\end{array}\right) \\
& \Gamma_{k, \pi}^{(4)}=\left(\begin{array}{ccc}
12 U_{k, \sigma \sigma} & 0 & 0 \\
0 & 4 U_{k, \sigma \sigma} & 0 \\
0 & 0 & 4 U_{k, \sigma \sigma}
\end{array}\right) \\
& \Gamma_{k, \sigma-\Delta}^{(4)}=\left(\begin{array}{ccc}
4 U_{k, \sigma \sigma}+8 \sigma^{2} U_{k, \sigma \sigma} & 8 \Delta \sigma U_{k, \sigma \Delta} & 0 \\
8 \Delta \sigma U_{k, \sigma \Delta} & 4 U_{k, \sigma \sigma}+8 \sigma^{2} U_{k, \sigma \sigma} & 0 \\
0 & 0 & 4 U_{k, \sigma \Delta}
\end{array}\right) \\
& \Gamma_{k, \Delta}^{(2)}=\left(\begin{array}{cccc}
4 U_{k, \sigma \Delta} & 0 & 0 & 0 \\
0 & 4 U_{k, \sigma \Delta} & 0 & 0 \\
0 & 0 & 4 U_{k, \sigma \Delta} & 0 \\
0 & 0 & 0 & 4 U_{k, \sigma \Delta}
\end{array}\right)
\end{aligned}
$$

The fermionic propagator is defined by

$$
G_{k_{\psi}}=\left(\begin{array}{cc}
\left(\Gamma_{k, \mathrm{rg}}^{(2)}-i \vec{p} r_{k}(\vec{p}) \otimes \mathbb{1}_{2 \times 2}\right)^{-1} & 0 \\
0 & \left(\Gamma_{k, \mathrm{~b}}^{(2)}-i \vec{p} r_{k}(\vec{p}) \otimes \mathbb{1}_{2 \times 2}\right)^{-1}
\end{array}\right)
$$

where $\left(\Gamma_{k, \mathrm{rg}}^{(2)}-i \vec{p} r_{k}(\vec{p}) \otimes \mathbb{1}_{2 \times 2}\right)^{-1}$ is given in Eq. (D.60) and $\left(\Gamma_{k, \mathrm{~b}}^{(2)}-i \vec{p} r_{k}(\vec{p}) \otimes \mathbb{1}_{2 \times 2}\right)^{-1}$ in Eq. (D.72). Furthermore we find

$$
\Gamma_{k, \bar{\psi} \psi \pi_{i}}^{(2,1)}=\left(\begin{array}{ccc}
h_{\phi} i \gamma_{5} \tau_{i} & 0 & 0 \\
0 & -h_{\phi} i \gamma_{5} \tau_{i} & 0 \\
0 & 0 & h_{\phi} i \gamma_{5} \tau_{i}
\end{array}\right)
$$


The strategy for the loop functions is now analogous to the previous section. First, we need to evaluate the field-space traces in Eq. (E.38). Then, we bring the resulting expression into the form of Eq. (E.11) such that we are able to distinguish between the two cases $k>|\vec{q}|$ and $k<|\vec{q}|$. Subsequently, we perform the Matsubara sum for the two different cases, which can be done analytically. However, the resulting expressions are very cumbersome, therefore we recommend doing this calculation using a symbolic computation language such as Mathematica. Finally, given a fixed external momentum $p$, we need to evaluate the remaining integral over the inner momenta $q$ numerically by using Eqs. (E.13) to (E.16). 


\section{Appendix F}

\section{Code Reference}

The code that has been implemented to produce the results of this thesis can be found on Github:

https://github.com/lakaschus/PhDThesis

\section{F.1 Minimization of the NJL-model Grand Poten- tial}

https://github.com/lakaschus/PhDThesis/tree/main/NJLModelMF

This link contains two python scripts that perform the minimization of the grand potentials for the CDW and the RKC Ansätze given in Eq. (3.27) and Eq. (3.30), respectively.

\section{F.2 Effective Potential of the QM model}

https://github.com/lakaschus/PhDThesis/tree/main/FRG/QMM

This directory contains three Jupyter notebooks for solving the flow equation of the QM model given in Eq. (4.54) using the standard finite-difference and the KT finite-volume scheme. It also contains a Jupyter notebook comparing both methods.

\section{F.3 Effective Potential of the QMD model}

https://github.com/lakaschus/PhDThesis/tree/main/FRG/QMDM

Here we find the numerical solver for Eq. (4.32) using the standard finitedifference and the KT finite-volume scheme. 


\section{F.4 Derivation of the Flow Equations for the Effec- tive Potential and the Two-Point Function for the QMD Model}

https://github.com/lakaschus/PhDThesis/blob/main/FRG/QMDM/nPoint FunctionDerivationThesis.nb

This Mathematica file contains the derivation for the effective potential of the QMD model. Even though we have already derived it in App. D, this script validates our analytic calculations. Furthermore, this script also contains the derivation for the flow equation of the pion two-point function which are needed for the stability analysis.

In case the reader does not have access to a Mathematica license, we recommend to use the Wolfram Player, which is a free tool that allows to view Mathematica scripts. 


\section{Bibliography}

[1] Ralf-Arno Tripolt et al. "Low-temperature behavior of the quark-meson model". In: Phys. Rev. D 97.3 (2018), p. 034022. DOI: 10.1103/PhysRevD . 97.034022. arXiv: 1709.05991 [hep-ph].

[2] N. Cabibbo and G. Parisi. "Exponential hadronic spectrum and quark liberation". In: Physics Letters B 59.1 (1975), pp. 67 -69. ISSN: 0370-2693. DOI: https://doi .org/10.1016/0370-2693(75)90158-6.

[3] Rolf Hagedorn. "Statistical thermodynamics of strong interactions at high energies". In: Nuovo Cimento, Suppl.3.CERN-TH-520 (1965), pp. 147186.

[4] W Nahm. "ANALYTICAL SOLUTION OF THE STATISTICAL BOOTSTRAP MODEL." In: Nucl. Phys. B45: No. 2, 525-53(1972). (Jan. 1972). DOI: 10.1016/0550-3213(72) 90257-X.

[5] A. Bazavov et al. "Chiral crossover in QCD at zero and non-zero chemical potentials". In: Phys. Lett. B 795 (2019), pp. 15-21. DOI: 10 . 1016/ j.physletb.2019.05.013. arXiv: 1812.08235 [hep-lat].

[6] Mark G. Alford, Krishna Rajagopal, and Frank Wilczek. "QCD at finite baryon density: Nucleon droplets and color superconductivity". In: Phys. Lett. B 422 (1998), pp. 247-256. DOI: 10 .1016/S0370-2693(98) 00051-3. arXiv: hep-ph/9711395.

[7] D Rischke. "The quark-gluon plasma in equilibrium". In: Progress in Particle and Nuclear Physics 52.1 (2004), 197-296. ISSN: 0146-6410. DOI: 10.1016/j . ppnp. 2003.09.002.

[8] Mark G. Alford et al. "Color superconductivity in dense quark matter". In: Rev. Mod. Phys. 80 (2008), pp. 1455-1515. DOI: 10.1103/RevModPhys . 80.1455. arXiv: 0709.4635 [hep-ph].

[9] Kenji Fukushima and Chihiro Sasaki. "The phase diagram of nuclear and quark matter at high baryon density". In: Prog. Part. Nucl. Phys. 72 (2013), pp. 99-154. DOI: 10 . 1016/ j . ppnp . 2013 . 05 . 003. arXiv: 1301.6377 [hep-ph].

[10] Kenji Fukushima and Tetsuo Hatsuda. "The phase diagram of dense QCD". In: Rept. Prog. Phys. 74 (2011), p. 014001. DOI: 10 . 1088/00344885/74/1/014001. arXiv: 1005.4814 [hep-ph].

[11] Jens O. Andersen, William R. Naylor, and Anders Tranberg. "Phase diagram of QCD in a magnetic field: A review". In: Rev. Mod. Phys. 88 (2016), p. 025001. DOI: 10 . 1103 / RevModPhys . 88 . 025001. arXiv: 1411.7176 [hep-ph]. 
[12] G. Grüner. "The dynamics of spin-density waves". In: Rev. Mod. Phys. 66 (1 1994), pp. 1-24. DOI: 10.1103/RevModPhys.66.1.

[13] Peter Fulde and Richard A. Ferrell. "Superconductivity in a Strong Spin-Exchange Field". In: Phys. Rev. 135 (3A 1964), A550-A563. DOI: 10.1103/PhysRev. 135.A550.

[14] A. W. Overhauser. "Structure of Nuclear Matter". In: Phys. Rev. Lett. 4 (8 1960), pp.415-418. DOI: 10.1103/PhysRevLett. 4.415.

[15] A.B. Migdal. "Stability of vacuum and limiting fields". In: Zh. Eksp. Teor. Fiz. 61 (1971), pp. 2209-2224.

[16] A. B. Migdal. " $\pi$ Condensation in Nuclear Matter". In: Phys. Rev. Lett. 31 (4 1973), pp. 257-260. DOI: 10.1103/PhysRevLett.31.257.

[17] Torleif Erik Oskar Ericson and W. Weise. Pions and Nuclei. Vol. 74. Oxford, UK: Clarendon Press, 1988. ISBN: 978-0-19-852008-5.

[18] Michael Thies and Konrad Urlichs. "Revised phase diagram of the Gross-Neveu model”. In: Phys. Rev. D 67 (2003), p. 125015. DOI: 10 . 1103/PhysRevD .67 .125015. arXiv: hep-th/0302092.

[19] Michael Thies. "Analytical solution of the Gross-Neveu model at finite density". In: Phys. Rev. D 69 (2004), p. 067703. DOI: 10.1103 / PhysRevD .69.067703. arXiv: hep-th/0308164.

[20] Wojciech Broniowski, Andrzej Kotlorz, and Marek Kutschera. “Quarks with a pion condensate. A new phase of matter". In: Acta Phys. Polon. B 22 (1991). Ed. by W. Czyz, pp. 145-166.

[21] Mariusz Sadzikowski. "Comparison of the non-uniform chiral and 2SC phases at finite temperatures and densities". In: Phys. Lett. B 642 (2006), pp. 238-243. DOI: 10.1016/ j . physletb. 2006 . 08 . 086. arXiv: hep-ph/0609186.

[22] Dominik Nickel. "How many phases meet at the chiral critical point?" In: Phys. Rev. Lett. 103 (2009), p. 072301. DOI: 10.1103/PhysRevLett. 103.072301. arXiv: 0902.1778 [hep-ph].

[23] Hiroaki Abuki, Daisuke Ishibashi, and Katsuhiko Suzuki. "Crystalline chiral condensates off the tricritical point in a generalized GinzburgLandau approach". In: Phys. Rev. D 85 (2012), p. 074002. DOI: 10.1103/ PhysRevD.85.074002. arXiv: 1109.1615 [hep-ph] .

[24] Achim Heinz et al. "Inhomogeneous condensation in effective models for QCD using the finite-mode approach". In: Phys. Rev. D 93.1 (2016), p. 014007. DOI: 10 . 1103 / PhysRevD . 93.014007. arXiv: 1508.06057 [hep-ph].

[25] M. Sadzikowski. "Coexistence of pion condensation and color superconductivity in two flavor quark matter". In: Phys. Lett. B 553 (2003), pp. 45-50. DOI: 10 . 1016/S0370-2693 (02 ) 03188-X. arXiv: hep-ph/ 0210065. 
[26] Daniel Nowakowski and Stefano Carignano. "Color-superconductivity and inhomogeneous chiral symmetry breaking in isospin-asymmetric quark matter". In: PoS MPCS2015 (2016), p. 010. DOI: 10 . 22323/1. 262.0010. arXiv: 1602.04798 [hep-ph].

[27] Dietrich Roscher, Jens Braun, and Joaquín E. Drut. "Phase structure of mass- and spin-imbalanced unitary Fermi gases". In: Phys. Rev. A 91.5 (2015), p. 053611. DOI: 10.1103 / PhysRevA . 91 . 053611. arXiv: 1501.05544 [cond-mat.quant-gas].

[28] Particle Data Group et al. "Review of Particle Physics". In: Progress of Theoretical and Experimental Physics 2020.8 (Aug. 2020). 083C01. ISSN: 2050-3911. DOI: 10 . 1093/ptep/ptaa104. eprint: https : //academic . oup . com/ptep/article-pdf / 2020/8/083C01/33653179/ptaa104 . pdf.

[29] Alexandre Deur, Stanley J. Brodsky, and Guy F. de Teramond. "The QCD Running Coupling". In: Nucl. Phys. 90 (2016), p. 1. DOI: 10.1016/ j.ppnp. 2016.04.003. arXiv: 1604.08082 [hep-ph].

[30] Jeffrey Goldstone, Abdus Salam, and Steven Weinberg. "Broken Symmetries". In: Phys. Rev. 127 (3 1962), pp. 965-970. DOI: 10.1103/PhysRev . 127.965.

[31] Mark Alford and Krishna Rajagopal. "Absence of two flavor color superconductivity in compact stars". In: JHEP 06 (2002), p. 031. DOI: 10.1088/1126-6708/2002/06/031. arXiv: hep-ph/0204001.

[32] Peter Hasenfratz and Julius Kuti. "The quark bag model". In: Physics Reports 40.2 (1978), pp. 75 -179. ISSN: 0370-1573. DOI: https : / doi . org/10 . 1016/0370-1573(78)90076-5.

[33] Carlos Naya and Paul Sutcliffe. "Skyrmions and clustering in light nuclei". In: Phys. Rev. Lett. 121.23 (2018), p. 232002. DOI: 10.1103 / PhysRevLett.121.232002. arXiv: 1811.02064 [hep-th].

[34] Boris A. Arbuzov, Mikhail K. Volkov, and Ivan V. Zaitsev. "NJL model derived from QCD". In: Int. J. Mod. Phys. A 21 (2006), pp. 5721-5742. DOI: 10.1142/S0217751X06033830. arXiv: hep-ph/0604051.

[35] M. K. Volkov and A. E. Radzhabov. "The Nambu-Jona-Lasinio model and its development". In: Phys. Usp. 49 (2006), pp. 551-561. DOI: 10 . 1070/PU2006v049n06ABEH005905. arXiv: hep-ph/0508263.

[36] Bernd-Jochen Schaefer and Jochen Wambach. "Renormalization group approach towards the QCD phase diagram". In: Phys. Part. Nucl. 39 (2008), pp. 1025-1032. DOI: 10.1134/S1063779608070083. arXiv: hep$\mathrm{ph} / 0611191$.

[37] Michael C. Birse and Manoj K. Banerjee. "Chiral model for nucleon and delta". In: Phys. Rev. D 31 (1 1985), pp. 118-127. DOI: $10.1103 /$ PhysRevD. 31.118.

[38] Murray Gell-Mann and M Levy. "The axial vector current in beta decay". In: Nuovo Cim. 16 (1960), p. 705. DOI: 10.1007/BF02859738. 
[39] F. Giacosa et al. "Scalar nonet quarkonia and the scalar glueball: Mixing and decays in an effective chiral approach". In: Phys. Rev. D 72 (2005), p. 094006. DOI: 10 . 1103 / PhysRevD . 72 . 094006. arXiv: hep$\mathrm{ph} / 0509247$.

[40] Denis Parganlija et al. "Meson vacuum phenomenology in a threeflavor linear sigma model with (axial-)vector mesons". In: Phys. Rev. D 87.1 (2013), p. 014011. DOI: 10 . 1103/PhysRevD . 87 .014011. arXiv: 1208.0585 [hep-ph].

[41] Florian Divotgey et al. "Low-energy limit of the extended Linear Sigma Model". In: Eur. Phys. J. A 54.1 (2018), p. 5. DOI: 10.1140/epja/i201812458-9. arXiv: 1605.05154 [hep-ph].

[42] Daiki Suenaga and Phillip Lakaschus. "Comprehensive study of mass modifications of light mesons in nuclear matter in the three-flavor extended linear $\sigma$ model". In: Phys. Rev. C 101.3 (2020), p. 035209. DOI: 10.1103/PhysRevC.101.035209. arXiv: 1908.10509 [nucl-th].

[43] Carleton DeTar and Teiji Kunihiro. "Linear sigma model with parity doubling". In: Phys. Rev. D 39 (9 1989), pp. 2805-2808. DOI: 10.1103/ PhysRevD . 39.2805.

[44] V. Dexheimer et al. "Neutron stars within the SU(2) parity doublet model". In: Eur. Phys. J. A 38 (2008), pp. 105-113. DOI: 10.1140/epja/ i2008-10652-0. arXiv: 0805.3301 [nucl-th].

[45] Stefan Scherer. "Introduction to chiral perturbation theory". In: Adv. Nucl. Phys. 27 (2003). Ed. by John W. Negele and E. W. Vogt, p. 277. arXiv: hep-ph/0210398.

[46] Kouji Kashiwa et al. "Critical endpoint in the Polyakov-loop extended NJL model". In: Phys. Lett. B 662 (2008), pp. 26-32. DOI: 10 . 1016/ j . physletb.2008.01.075. arXiv: 0710.2180 [hep-ph].

[47] Bernd-Jochen Schaefer, Jan M. Pawlowski, and Jochen Wambach. "The Phase Structure of the Polyakov-Quark-Meson Model". In: Phys. Rev. D 76 (2007), p. 074023. DOI: 10.1103 / PhysRevD . 76 . 074023. arXiv: 0704.3234 [hep-ph].

[48] J. D. Walecka. "A Theory of highly condensed matter". In: Annals Phys. 83 (1974), pp. 491-529. DOI: 10.1016/0003-4916 (74)90208-5.

[49] Brian D. Serot and John Dirk Walecka. "The Relativistic Nuclear Many Body Problem". In: Adv. Nucl. Phys. 16 (1986), pp. 1-327.

[50] M. Benayoun et al. "A Global Treatment Of VMD Physics Up To The phi: I. e+ e- Annihilations, Anomalies And Vector Meson Partial Widths". In: Eur. Phys. J. C 65 (2010), pp. 211-245. DOI: 10.1140/epjc/s10052009-1197-6. arXiv: 0907.4047 [hep-ph].

[51] Aneesh V. Manohar. "Introduction to Effective Field Theories". In: Les Houches Lect. Notes 108 (2020). DOI: 10 . 1093 / oso / 9780198855743. 003.0002. arXiv: 1804.05863 [hep-ph]. 
[52] C.P. Burgess. "Introduction to Effective Field Theory". In: Ann. Rev. Nucl. Part. Sci. 57 (2007), pp. 329-362. DOI: 10 .1146/annurev . nucl . 56.080805.140508. arXiv: hep-th/0701053.

[53] H. Georgi. "Effective field theory". In: Ann. Rev. Nucl. Part. Sci. 43 (1993), pp. 209-252. DOI: 10.1146/annurev .ns .43.120193.001233.

[54] Y. Nambu and G. Jona-Lasinio. “Dynamical Model of Elementary Particles Based on an Analogy with Superconductivity. I". In: Phys. Rev. 122 (1 1961), pp. 345-358. DOI: 10.1103/PhysRev.122.345.

[55] Y. Nambu and G. Jona-Lasinio. “Dynamical Model of Elementary Particles Based on an Analogy with Superconductivity. II". In: Phys. Rev. 124 (1 1961), pp. 246-254. DOI: 10.1103/PhysRev.124.246.

[56] Michael Buballa. "NJL model analysis of dense quark matter". In: Phys. Rept. 407 (2005), pp. 205-376. DOI: 10 . 1016/ j . physrep. 2004. 11.004. arXiv: hep-ph/0402234.

[57] S. P. Klevansky. “The Nambu-Jona-Lasinio model of quantum chromodynamics". In: Rev. Mod. Phys. 64 (3 1992), pp. 649-708. DOI: 10 . 1103/RevModPhys . 64.649.

[58] D.U. Jungnickel and C. Wetterich. "Effective action for the chiral quarkmeson model". In: Phys. Rev. D 53 (1996), pp. 5142-5175. DOI: 10 . 1103/PhysRevD.53.5142. arXiv: hep-ph/9505267.

[59] N. Tetradis. "The Quark meson model and the phase diagram of two flavor QCD". In: Nucl. Phys. A 726 (2003), pp. 93-119. DOI: 10 . 1016/ S0375-9474 (03) 01624-5. arXiv: hep-th/0303244.

[60] Ralf-Arno Tripolt et al. "Spectral Functions for the Quark-Meson Model Phase Diagram from the Functional Renormalization Group". In: Phys. Rev. D 89.3 (2014), p. 034010. DOI: 10 . 1103/ PhysRevD . 89 . 034010. arXiv: 1311.0630 [hep-ph].

[61] Tohru Eguchi. "New approach to collective phenomena in superconductivity models". In: Phys. Rev. D 14 (10 1976), pp. 2755-2763. DOI: 10.1103/PhysRevD.14.2755.

[62] Keiji Kikkawa. "Quantum Corrections in Superconductor Models". In: Progress of Theoretical Physics 56.3 (Sept. 1976), pp. 947-955. ISSN: 0033-068X. DOI: 10 . 1143/PTP . 56 . 947. eprint: https : / / academic . oup. com/ptp/article-pdf/56/3/947/5455249/56-3-947.pdf.

[63] Shotaro Imai, Hiroshi Toki, and Wolfram Weise. "Quark-Hadron Matter at Finite Temperature and Density in a Two-Color PNJL model". In: Nucl. Phys. A 913 (2013), pp. 71-102. DOI: 10 . 1016/j . nuclphysa. 2013.06.001. arXiv: 1210.1307 [nucl-th].

[64] Shotaro Imai. "Quark-Hadron matter in two color Nambu-Jona-Lasinio model". PhD thesis. Osaka U., 2013.

[65] Naseemuddin Khan. "Interplay of Mesonic and Baryonic Degrees of Freedom in Quark Matter". PhD thesis. Jan. 2015. 
[66] Nils Strodthoff, Bernd-Jochen Schaefer, and Lorenz von Smekal. “Quarkmeson-diquark model for two-color QCD". In: Phys. Rev. D 85 (2012), p. 074007. DOI: 10.1103 / PhysRevD . 85 .074007. arXiv: 1112 . 5401 [hep-ph].

[67] Nils Strodthoff. "Critical Phenomena in the Phase Diagrams of QCDlike Theories". PhD thesis. Darmstadt, Tech. Hochsch., 2013.

[68] Nils Strodthoff and Lorenz Von Smekal. "Polyakov-quark-meson-diquark model for two-color QCD”. In: Physics Letters B 731 (Apr. 2014), pp. 350357. DOI: $10.1016 / j$. physletb. 2014.03.008.

[69] Naseemuddin Khan et al. "The Phase Diagram of QC2D from Functional Methods". In: (Dec. 2015). arXiv: 1512.03673 [hep-ph].

[70] Niklas Cichutek, Florian Divotgey, and Jürgen Eser. "Fluctuation-induced higher-derivative couplings and infrared dynamics of the Quark-MesonDiquark Model". In: Phys. Rev. D 102.3 (2020), p. 034030. DOI: 10 . 1103/PhysRevD .102.034030. arXiv: 2006.12473 [hep-ph].

[71] J.B. Kogut, Misha A. Stephanov, and D. Toublan. “On two color QCD with baryon chemical potential". In: Phys. Lett. B 464 (1999), pp. 183191. DOI: $10.1016 / \mathrm{S} 0370-2693$ (99)00971-5. arXiv: hep-ph/9906346.

[72] J.B. Kogut et al. "QCD - like theories at finite baryon density". In: Nucl. Phys. B 582 (2000), pp. 477-513. DOI: 10.1016/S0550-3213(00)00242X. arXiv: hep-ph/0001171.

[73] Simon Hands et al. "Symmetries and spectrum of SU(2) lattice gauge theory at finite chemical potential". In: Nucl. Phys. B 558 (1999), pp. 327346. DOI: 10.1016/S0550-3213 (99)00364-8. arXiv: hep-lat/9902034.

[74] Simon Hands et al. "Diquark condensation in dense adjoint matter". In: Eur. Phys. J. C 22 (2001), pp. 451-461. DOI: 10.1007/s100520100836. arXiv: hep-lat/0109029.

[75] Phillip Lakaschus, Michael Buballa, and Dirk H. Rischke. "Competition of inhomogeneous chiral phases and two-flavor color superconductivity in the NJL model". In: (Dec. 2020). arXiv: 2012 . 07520 [hep-ph].

[76] Andreas Schmitt. Introduction to Superfluidity: Field-theoretical approach and applications. Vol. 888. 2015. ISBN: 978-3-319-07946-2,978-3-319-079479. DOI: $10.1007 / 978-3-319-07947-9$. arXiv: 1404.1284 [hep-ph] .

[77] Dominik Nickel. "Inhomogeneous phases in the Nambu-Jona-Lasino and quark-meson model". In: Phys. Rev. D 80 (2009), p. 074025. DOI: 10.1103/PhysRevD.80.074025. arXiv: 0906.5295 [hep-ph].

[78] Stefano Carignano, Dominik Nickel, and Michael Buballa. "Influence of vector interaction and Polyakov loop dynamics on inhomogeneous chiral symmetry breaking phases". In: Phys. Rev. D 82 (2010), p. 054009. DOI: 10.1103/PhysRevD .82.054009. arXiv: 1007.1397 [hep-ph]. 
[79] J.I. Kapusta and Charles Gale. Finite-temperature field theory: Principles and applications. Cambridge Monographs on Mathematical Physics. Cambridge University Press, 2011. ISBN: 978-0-521-17322-3, 978-0-52182082-0, 978-0-511-22280-1. DOI: 10.1017/CB09780511535130.

[80] F. Dautry and E.M. Nyman. "PION CONDENSATION AND THE SIGMA MODEL IN LIQUID NEUTRON MATTER". In: Nucl. Phys. A 319 (1979), pp. 323-348. DOI: 10.1016/0375-9474 (79) 90518-9.

[81] Marek Kutschera, Wojciech Broniowski, and Andrzej Kotlorz. "Quark Matter With Pion Condensate in an Effective Chiral Model". In: Nucl. Phys. A 516 (1990), pp. 566-588. DOI: 10.1016/0375-9474 (90) 901289.

[82] S. P. Klevansky. "The Nambu-Jona-Lasinio model of quantum chromodynamics". In: Rev. Mod. Phys. 64 (3 1992), pp. 649-708. DOI: 10 . 1103/RevModPhys .64.649.

[83] Stefano Carignano and Michael Buballa. "Inhomogeneous islands and continents in the Nambu-Jona-Lasinio model". In: Acta Phys. Polon. Supp. 5 (2012). Ed. by L. Turko, pp. 641-658. DOI: 10 . 5506/APhysPolBSupp. 5.641. arXiv: 1111.4400 [hep-ph].

[84] E. Nakano and T. Tatsumi. "Chiral symmetry and density wave in quark matter". In: Phys. Rev. D 71 (2005), p. 114006. DOI: 10.1103 / PhysRevD.71.114006. arXiv: hep-ph/0411350.

[85] L.P. Kadanoff. "Scaling laws for Ising models near T(c)". In: Physics Physique Fizika 2 (1966), pp. 263-272. DOI: 10.1103/PhysicsPhysiqueFizika. 2.263.

[86] K.G. Wilson. "PROBLEMS IN PHYSICS WITH MANY SCALES OF LENGTH". In: Sci. Am. 241 (1979), pp. 140-157. DOI: 10.1038/scientificamerican0879158.

[87] Vincent Dumoulin and Francesco Visin. A guide to convolution arithmetic for deep learning. 2018. arXiv: 1603.07285 [stat.ML].

[88] Kenneth G. Wilson. "The renormalization group: Critical phenomena and the Kondo problem". In: Rev. Mod. Phys. 47 (4 1975), pp. 773-840. DOI: 10.1103/RevModPhys .47.773.

[89] Christof Wetterich. "Exact evolution equation for the effective potential". In: Phys. Lett. B 301 (1993), pp. 90-94. DOI: 10 . 1016 / 0370 2693 (93)90726-X. arXiv: 1710.05815 [hep-th] .

[90] Juergen Berges, Nikolaos Tetradis, and Christof Wetterich. “Nonperturbative renormalization flow in quantum field theory and statistical physics". In: Phys. Rept. 363 (2002), pp. 223-386. DOI: 10.1016/S03701573(01) 00098-9. arXiv: hep-ph/0005122.

[91] Holger Gies. "Introduction to the functional RG and applications to gauge theories". In: Lect. Notes Phys. 852 (2012), pp. 287-348. DOI: 10. 1007/978-3-642-27320-9\_6. arXiv: hep-ph/0611146. 
[92] P. Kopietz, L. Bartosch, and F. Schütz. Introduction to the Functional Renormalization Group. Lecture Notes in Physics. Springer Berlin Heidelberg, 2010. ISBN: 9783642050947.

[93] N. Dupuis et al. "The nonperturbative functional renormalization group and its applications". In: (June 2020). arXiv: 2006.04853 [cond-mat . stat-mech] .

[94] Daniel F. Litim. "Optimized renormalization group flows". In: Phys. Rev. D 64 (2001), p. 105007. DOI: 10.1103/PhysRevD.64.105007. arXiv: hep-th/0103195.

[95] Stefano Carignano, Michael Buballa, and Bernd-Jochen Schaefer. "Inhomogeneous phases in the quark-meson model with vacuum fluctuations". In: Phys. Rev. D 90.1 (2014), p. 014033. DOI: 10.1103/PhysRevD . 90.014033. arXiv: 1404.0057 [hep-ph].

[96] Ralf-Arno Tripolt. "Spectral Functions and Transport Coefficients from the Functional Renormalization Group". PhD thesis. Darmstadt, Tech. Hochsch., 2015.

[97] Eduardo Grossi and Nicolas Wink. "Resolving phase transitions with Discontinuous Galerkin methods". In: (Mar. 2019). arXiv: 1903.09503 [hep-th].

[98] Eduardo Grossi et al. "Shocks and quark-meson scatterings at large density". In: (Feb. 2021). arXiv: 2102.01602 [hep-ph].

[99] Adrian Koenigstein et al. "Numerical fluid dynamics for FRG flow equations: Zero-dimensional QFTs as numerical test cases - Part I: The $O(N)$ model". In: (Aug. 2021). arXiv: 2108.02504 [cond-mat. stat-mech] .

[100] Adrian Koenigstein et al. "Numerical fluid dynamics for FRG flow equations: Zero-dimensional QFTs as numerical test cases - Part II: Entropy production and irreversibility of RG flows". In: (Aug. 2021). arXiv: 2108.10085 [cond-mat.stat-mech].

[101] Martin J. Steil and Adrian Koenigstein. "Numerical fluid dynamics for FRG flow equations: Zero-dimensional QFTs as numerical test cases - Part III: Shock and rarefaction waves in RG flows reveal limitations of the $N \rightarrow \infty$ limit in $O(N)$-type models". In: (Aug. 2021). arXiv: 2108.04037 [cond-mat.stat-mech].

[102] Eitan Tadmor Alexander Kurganov. "New High-Resolution Central Schemes for Nonlinear Conservation Laws and Convection-Diffusion Equations". In: Journal of Computational Physics 160 (2001).

[103] Alexander Kurganov and Eitan Tadmor. "Solution of two-dimensional Riemann problems for gas dynamics without Riemann problem solvers". In: Numerical Methods for Partial Differential Equations 18.5 (2002), pp. 584608. DOI: https : / / doi . org/10 . 1002 / num . 10025. eprint: https : //onlinelibrary.wiley.com/doi/pdf/10.1002/num.10025.

[104] Alexander Kurganov and G. Petrova. "A third-order semi-discrete genuinely multidimensional central scheme for hyperbolic conservation laws and related problems". In: Numerische Mathematik 88 (June 2001). DOI: $10.1007 /$ PL00005455. 
[105] Renan Câmara Pereira, Rainer Stiele, and Pedro Costa. "Functional renormalization group study of the critical region of the quark-meson model with vector interactions". In: Eur. Phys. J. C 80.8 (2020), p. 712. DOI: 10.1140/epjc/s10052-020-8296-9. arXiv: 2003.12829 [hep-ph] .

[106] Jürgen Eser et al. "Low-energy limit of the $O(4)$ quark-meson model from the functional renormalization group approach". In: Phys. Rev. D 98.1 (2018), p. 014024. DOI: 10.1103/PhysRevD . 98 .014024. arXiv: 1804.01787 [hep-ph].

[107] Thomas D . Cohen. "Functional integrals for QCD at nonzero chemical potential and zero density". In: Phys. Rev. Lett. 91 (2003), p. 222001. DOI: 10.1103/PhysRevLett.91.222001. arXiv: hep-ph/0307089.

[108] Gergely Markó, Urko Reinosa, and Zsolt Szép. “Bose-Einstein condensation and Silver Blaze property from the two-loop $\Phi$-derivable approximation". In: Phys. Rev. D 90.12 (2014), p. 125021. DOI: 10.1103/ PhysRevD.90.125021. arXiv: 1410.6998 [hep-ph].

[109] Ken-Ichi Aoki et al. "Rapidly converging truncation scheme of the exact renormalization group". In: Prog. Theor. Phys. 99 (1998), pp. 451466. DOI: 10.1143/PTP. 99.451. arXiv: hep-th/9803056.

[110] Jens Braun, Marc Leonhardt, and Jan M. Pawlowski. "Renormalization group consistency and low-energy effective theories". In: SciPost Phys. 6.5 (2019), p. 056. DOI: 10.21468/SciPostPhys . 6.5.056. arXiv: 1806.04432 [hep-ph].

[111] Oliver Schnetz, Michael Thies, and Konrad Urlichs. "Full phase diagram of the massive Gross-Neveu model". In: Annals Phys. 321 (2006), pp. 2604-2637. DOI: $10.1016 / \mathrm{j}$. aop . 2005 . 12 .007. arXiv: hep-th/ 0511206.

[112] Stefano Carignano, Marco Schramm, and Michael Buballa. "Influence of vector interactions on the favored shape of inhomogeneous chiral condensates". In: Phys. Rev. D 98.1 (2018), p. 014033. DOI: $10.1103 /$ PhysRevD.98.014033. arXiv: 1805.06203 [hep-ph].

[113] Nino M. Bratovic, Tetsuo Hatsuda, and Wolfram Weise. "Role of Vector Interaction and Axial Anomaly in the PNJL Modeling of the QCD Phase Diagram". In: Phys. Lett. B 719 (2013), pp. 131-135. DOI: 10 . 1016/j . physletb.2013.01.003. arXiv: 1204.3788 [hep-ph].

[114] Daniel Nowakowski et al. "Inhomogeneous chiral symmetry breaking phases in isospin-asymmetric matter". In: Compact Stars in the QCD Phase Diagram IV. June 2015. arXiv: 1506.04260 [hep-ph].

[115] Jean-Paul Blaizot, Ramon Mendez-Galain, and Nicolas Wschebor. "Non perturbative renormalisation group and momentum dependence of n-point functions (I)". In: Phys. Rev. E 74 (2006), p. 051116. DOI: 10 . 1103/PhysRevE. 74.051116. arXiv: hep-th/0512317. 
[116] Jean-Paul Blaizot, Ramon Mendez-Galain, and Nicolas Wschebor. "Non perturbative renormalization group and momentum dependence of n-point functions. II." In: Phys. Rev. E 74 (2006), p. 051117. DOI: 10. 1103/PhysRevE. 74.051117. arXiv: hep-th/0603163.

[117] Z.G. Wang, S.L. Wan, and W.M. Yang. "A New approach for calculating the Nambu-Gorkov propagator in color superconductivity theory". In: (Aug. 2005). arXiv: hep-ph/0508302. 


\section{Contribution to Chapter 3}

Chapter 3 is based on Ref. [75] in collaboration with Dirk H. Rischke and Michael Buballa. My contribution to this publication is as follows: I have done most of the analytic calculations, performed the numerical implementation for the minimzation of the grand potential, created the figures and prepared the first draft of the paper. However, Michael Buballa drastically improved and simplified my analytic calculation by providing the calculation steps from Eq. (3.10) to (3.21). Both Dirk Rischke and Michael Buballa helped to finalize the manuscript for the publication. 



\section{Danksagung}

An erster Stelle möchte ich mich bei Prof. Dr. Dirk-Hermann Rischke bedanken, der mir die Möglichkeit gegeben hat, meinen Traum zu verwirklichen, auf dem Gebiet der Quantenfeldtheorie forschen zu können. Ich bin insbesondere dankbar für seine Unterstützung und Ratschläge, aber auch dafür, dass er mir genug Freiheit gegeben hat, eigene Ideen zu verfolgen. Auch bin ich dankbar für die wertvollen fachlichen und sprachlichen Bemerkungen zur vorliegenden Doktorarbeit, was die Arbeit meines Erachtens deutlich aufgewertet hat.

Als nächstes möchte ich mich bei Prof. Dr. Jürgen Schaffner-Bielich bedanken, der sich bereitwillig dazu erklärt, meine Doktorarbeit zu begutachten.

Des Weiteren bedanke ich mich vielmals bei Priv. Doz. Dr. Michael Buballa für die zahlreichen Diskussionen und den hilfreichen Input insbesondere zum Thema der Inhomogenenen Phasen.

Außerdem möchte ich Dr. Ralf-Arno Tripolt hervorheben, da er mir geholfen hat, ein konkretes Thema zum FRG-Teil dieser Arbeit zu finden und mich dabei betreut hat. Seine Ratschläge waren von großer Hilfe für mich und dafür bin ich ihm sehr dankbar.

Ich möchte diese Gelegenheit auch nutzen, um insbesondere zwei meiner Kommilitonen hervorzuheben, nämlich Niklas Cichutek und Lutz Kiefer, mit denen ich während meiner Doktorarbeit nicht nur regelmäßig zusammengearbeitet haben, sondern auch viel Spaß gehabt hatte. Sie haben mit großer Sorgfalt meine Doktorarbeit gelesen und mir äußerst wertvolle Verbesserungsvorschläge gegeben.

Ebenso bedanke ich mich bei Abdol Sabor Salek, Alexander Stegemann, Dominik Kraatz, Justin Mauldin und die gesamte Chiral Group für wervolle physikalische Diskussionen und eine tolle Zeit im Institut.

Zum Abschluss möchte ich mich bei allen meinen Freunden und meiner Familie bedanken, die immer ein offenes Ohr für meine Probleme und Sorgen haben. Dabei möchte insbesondere Ignacia Deisen hervorheben, die mich in vielerlei Hinsicht unterstützt hat! Mein größter Dank geht jedoch an meine Verlobte Yao Yao (姚瑶), denn ohne ihre Unterstützung und Liebe hätte ich eine sehr schwere Zeit gehabt. 谢谢你的帮助。我非常爱你! Ich möchte mich auch bei 果果 bedanken, da sie mir stets ein Lächeln auf den Lippen zaubert! 